\title{
A Discrete Element Model (DEM) for Predicting Apple Damage during Handling
}

\author{
by
}

Otto Carl Scheffler

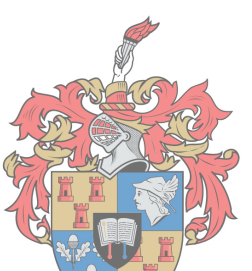

Thesis presented in partial fulfilment of the requirements for the degree of Master of Engineering (Mechanical) in the Faculty of Engineering at StellenboschsUniversity

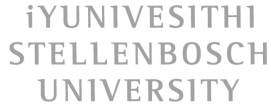

"The financial assistance of the National Research Foundation (NRF) towards this research is hereby acknowledged. Opinions expressed and conclusions arrived at, are those of the author and are not necessarily to be attributed to the NRF."

Supervisor: Prof CJ Coetzee

Co-supervisor: Prof UL Opara

March 2018 


\section{DECLARATION}

By submitting this thesis electronically, I declare that the entirety of the work contained therein is my own, original work, that I am the sole author thereof (save to the extent explicitly otherwise stated), that reproduction and publication thereof by Stellenbosch University will not infringe any third party rights and that I have not previously in its entirety or in part submitted it for obtaining any qualification.

Date: March 2018

Copyright ( 2018 Stellenbosch University All rights reserved 


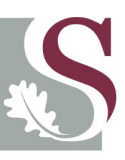

UNIVERSITEIT•STELLENBOSCH•UNIVERSITY jou kennisvennoot - your knowledge partner

\section{Plagiaatverklaring / Plagiarism Declaration}

1. Plagiaat is die oorneem en gebruik van die idees, materiaal en ander intellektuele eiendom van ander persone asof dit jou eie werk is.

Plagiarism is the use of ideas, material and other intellectual property of another's work and to present is as my own.

2. Ek erken dat die pleeg van plagiaat ' $n$ strafbare oortreding is aangesien dit ' $n$ vorm van diefstal is.

I agree that plagiarism is a punishable offence because it constitutes theft.

3. Ek verstaan ook dat direkte vertalings plagiaat is.

I also understand that direct translations are plagiarism.

4. Dienooreenkomstig is alle aanhalings en bydraes vanuit enige bron (ingesluit die internet) volledig verwys (erken). Ek erken dat die woordelikse aanhaal van teks sonder aanhalingstekens (selfs al word die bron volledig erken) plagiaat is.

Accordingly all quotations and contributions from any source whatsoever (including the internet) have been cited fully. I understand that the reproduction of text without quotation marks (even when the source is cited) is plagiarism.

5. Ek verklaar dat die werk in hierdie skryfstuk vervat, behalwe waar anders aangedui, my eie oorspronklike werk is en dat ek dit nie vantevore in die geheel of gedeeltelik ingehandig het vir bepunting in hierdie module/werkstuk of ' $n$ ander module/werkstuk nie.

I declare that the work contained in this assignment, except where otherwise stated, is my original work and that I have not previously (in its entirety or in part) submitted it for grading in this module/assignment or another module/assignment.

\begin{tabular}{|c|c|}
\hline & \\
Studentenommer / Student number & Handtekening / Signature \\
\hline Voorletters en van / Initials and surname & \\
\hline
\end{tabular}




\title{
ABSTRACT
}

\section{A Discrete Element Model (DEM) for Predicting Apple Damage during Handling}

\author{
Otto Carl Scheffler \\ Department of Mechanical and Mechatronic Engineering, \\ University of Stellenbosch, \\ Private Bag X1, Matieland 7602, South Africa. \\ Thesis: MEng (Mech)
}

March 2018

The Discrete Element Method (DEM) is used outside of its original purpose within the field of rock mechanics and applied to the agricultural sector. Apples are globally one of the most widely traded fruit and still suffer from significant mechanical injury in the form of bruise damage. Apple damage accounts for a significant proportion of financial losses in the postharvest handling of fresh produce.

DEM allows for individual particle contacts and the dynamic mechanical behaviour of a group of particles to be studied through collisions. Successfully applying this method to fresh produce, such as apples, allows for future investigations into postharvest mechanical damage to be performed on a range of fruit and vegetables.

A contact model that is capable of closely replicating the viscoelastic nature of apples was developed and applied. The mechanical material parameters required for the implementation of the viscoelastic model were successfully derived through an error minimisation algorithm and the use of a pendulum impactor. The accuracy of the contact force model was dependent on definite representation of the effective damping constant and was less sensitive to the elastic stiffness. 
Bruise damage models (in the form of bruise volume, bruise area and bruise depth) were coupled to the impact forces available through DEM. Bruise formations resulting from multiple impacts and variable time durations between impacts were studied. The resulting models were implemented in the numerical environment. A detailed particle shape representation along with a realistic contact point loading scheme was implemented in the DEM model. Overlapping bruises were studied and accounted for on a post-process level.

The resulting DEM model was successfully validated with the use of numerous physical experiments. Experimental validation commenced at a single specimen contact level and expanded into realistic situations, with up to 90 specimens, representative of conditions that may be experienced in practice. The model was extended to include run-time bruise visualisation during the DEM simulations.

Good correlation between the experimental and numerical results were achieved. Quantitatively, the model succeeded in accurately predicting the contact forces typically experienced by apples to within $11 \%$. The model predicted the mean bruise damage of a single apple for realistic situations within an accuracy of $50 \%$ in terms of mean bruise volume, $35 \%$ for bruise area and $30 \%$ for bruise depth. Qualitatively good agreement of the dynamic mechanical behaviour predicted by the model and the experiments was achieved. 


\title{
OPSOMMING
}

\section{'n Diskrete Element Model (DEM) vir die Voorspelling van Appelskade tydens Hantering}

\author{
Otto Carl Scheffler \\ Departement Meganiese en Megatroniese Ingenieurswese, \\ Universiteit van Stellenbosch, \\ Privaatsak X1, Matieland 7602, Suid Afrika. \\ Tesis: Mlng (Meg) \\ Maart 2018
}

Die Diskrete Element Metode (DEM) word aangewend buite die oorspronklike veld van rotsmeganika en toegepas in die landboumilieu. Appels is van die vrugte waarmee daar wêreldwyd die meeste handel gedryf word en doen steeds in die proses meganiese skade op. Appelskade as gevolg van die hantering tydens die na-oes, veroorsaak aansienlike finansiële verliese.

Met behulp van DEM kan individuele partikel kontakte, asook die meganiese gedrag van 'n groep partikels deeglik bestudeer word. Die suksesvolle toepassing van hierdie metode op vars produkte, soos appels, veroorloof (verdere) navorsing op meganiese skade tydens die na-oes van 'n verskeidenheid vrugte en groente.

'n Kontakmodel wat die visko-elastiese gedrag van appels noukeurig kan voorspel, is toegepas. Die meganiese parameters wat benodig word vir die toepassing van die model is suksesvol afgelei met behulp van ' $n$ foutminimeringsalgoritme, sowel as 'n pendulumimpakapparaat. Dit blyk dat die akkuraatheid van die kontakmodel meer afhanklik van die effektiewe dempingskonstante as die elastiese styfheid is.

Appelkneusingsmodelle (kneusvolume, kneusarea en kneusdiepte) is gekoppel aan impakkragte, soos beskikbaar gestel deur die gebruik van DEM. Die vorming 
van kneusing as gevolg van herhaaldelike impak en die tydsverloop tussen die impakte wat lei tot kneusing is bestudeerder. 'n Gedetailleerde partikel vormvoorstelling, tesame met ' $n$ realistiese kontakpunt laaiskema is geïmplementeer binne die DEM model. Modelle is gevolglik ontwikkel en daarna numeries toegepas. Die effek van oorvleuelende kneusings is ook bestudeer en in ag geneem op naprosseseringsvlak.

Die DEM model wat tot stand kom, is suksesvol gevalideer met behulp van talle fisiese eksperimente. Eksperimentele validasie het begin op ' $n$ enkel monster kontakvlak en is uitgebrei tot realistiese situasies wat in die praktyk sal voorkom. Die model word dan nog verder verfyn om kneusingformasie visueel voor te stel soos dit tydens die DEM simulasie plaasvind.

Uitstekende korrelasies is tussen die eksperimentele en numeriese resultate behaal. Die model het daarin geslaag om die kontakkragte wat tipies deur appels ondervind word met 'n $11 \%$ akkuraatheid te voorspel. Die model voorspel die gemiddelde skadeverlies, wat enkel appels ondervind in realistiese situasies, met 'n akkuraatheid van $50 \%$ met betrekking tot kneusvolume, $35 \%$ vir kneusarea en $30 \%$ vir kneusdiepte. 'n Uitstekende ooreenkoms is kwalitatief tussen die dinamiese gedrag van die model en die eksperimente behaal. 


\section{ACKNOWLEDGEMENTS}

The undertaking that took place over the past two years would not have been possible or successful without the love, support, care and advice from the author's mother and father. Words cannot describe the love and appreciation that is extended to both of you.

Many thanks are extended to Professor Lou-Marie Kruger for her words of advice. Without her counsel the past two years would not have been possible.

The author wishes to thank the project's study leader Professor Corné Coetzee. Much appreciation is extended for his guidance and knowledge conferred during the course of the project. Without his assistance and expertise, the successful completion of the study would not have been realised. Furthermore, his financial assistance is much appreciated.

Many thanks to the author's friends Matthys Du Toit and André Graaff. Thank you for supporting me, listening and offering advice throughout this endeavour. Furthermore, gratitude is also extended to Jean-Gerard de la Bat and JG Bergenthuin for their fellowship during the progression of the project.

Gratitude is extended to Hannes Swart for his assistance with the vibration tests and load cell substantiation. I also wish to acknowledge Mr Cobus Zietsman, Julian Stanfliet and the rest of the workshop staff for their assistance. Additionally, gratitude is extended to Roux Groenewald for his assistance with the attainment of the test specimens.

The author also wishes to thank Dr J E Hoffmann who encouraged the undertaking of postgraduate studies.

Recognition is given to Professor L Opara for his consultation during the study.

Considerable gratitude is given to the Lord Jesus Christ for his spiritual teachings and abundant blessings. 


\section{TABLE OF CONTENTS}

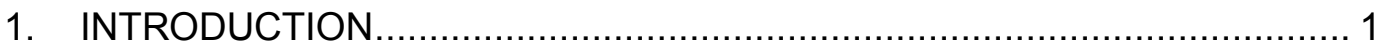

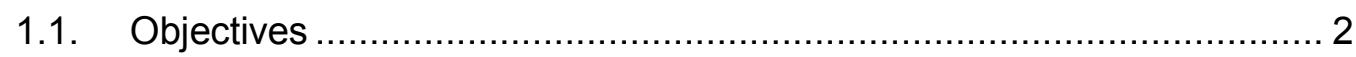

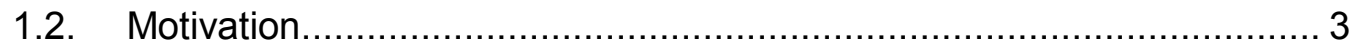

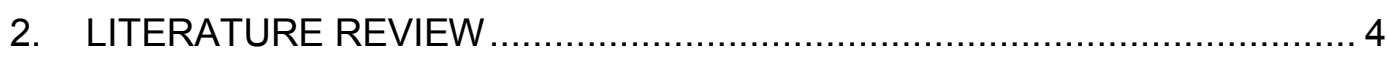

2.1. Apples: Background and Bruising .................................................... 4

2.2. Postharvest Areas Responsible for Apple Bruising............................ 7

2.3. Material Properties of Biological Materials ........................................ 8

2.4. Investigations into the Bruising of Fruit and Vegetables .................. 11

2.5. The Discrete Element Method …............................................. 13

2.5.1. Shape Representation Approaches...................................... 15

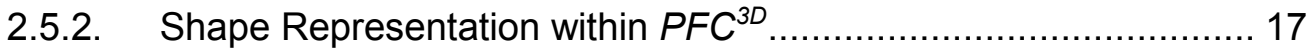

2.6. Contact Force Models for Viscoelastic Materials ............................ 17

2.7. Limitations of Previous Studies ...................................................... 20

3. CONTACT MODEL AND PARAMETER ACQUIREMENT ........................ 22

3.1. The Contact Model ..................................................................... 22

3.2. Experimental Set-Up and Procedures .......................................... 24

3.2.1. Quasi-Static Compression Tests ............................................ 24

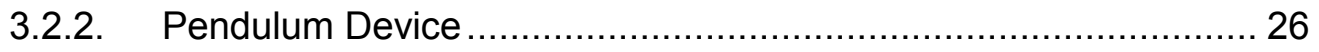

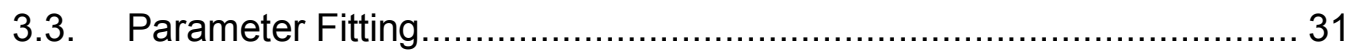

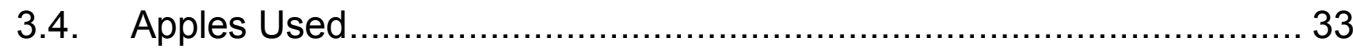

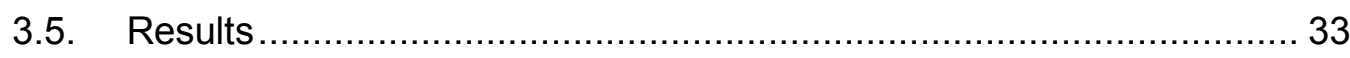

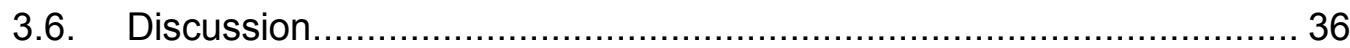

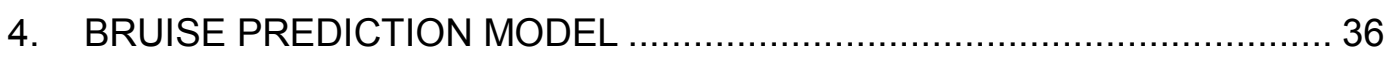

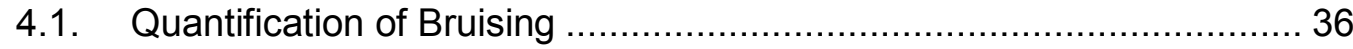

4.2. Experimental Set-Up and Procedure .......................................... 38

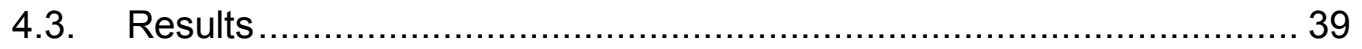


4.4. Onset of Apple Bruising ................................................................. 41

4.5. Effect of Multiple Contacts and Time between Impacts .................... 42

4.6. Resulting Bruise Damage Models ................................................. 44

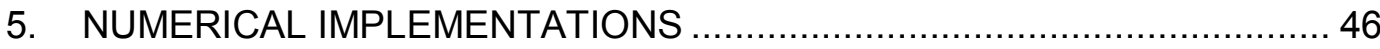

5.1. User Defined PFC ${ }^{3 D}$ Model Components....................................... 46

5.2. Representation of Particle Shape and Size .................................... 46

5.3. Contact Model Implementation..................................................... 50

5.4. Single Point Contact Assignment …........................................... 52

5.5. Force and Bruise Damage Coupling........................................... 56

5.6. Overlap Formulation................................................................... 57

6. EXPERIMENTAL VALIDATION OF THE DEM BRUISING MODEL ...........61 61

6.1. Validation Procedure ....................................................................... 61

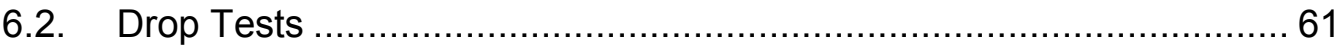

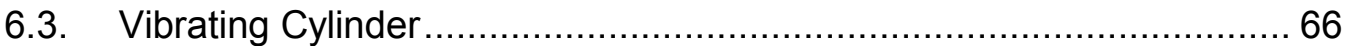

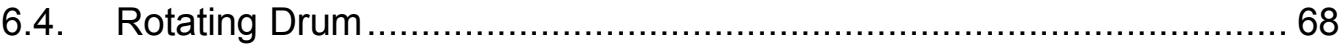

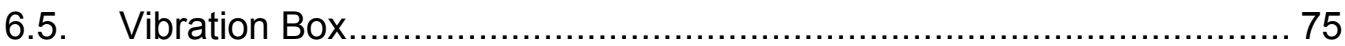

6.6. Bruise Visualisation and Shape Modelling .................................... 80

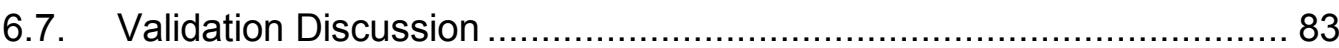

7. CONCLUSION AND RECOMMENDATIONS ….................................. 84

7.1. Contact Model Outcomes............................................................ 84

7.2. Bruise Damage Investigation......................................................... 85

7.3. Numerical Implementation and Validation ...................................... 85

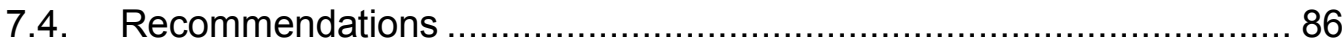

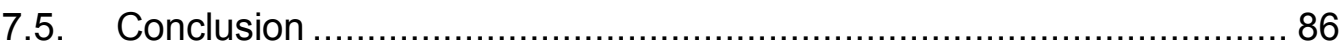

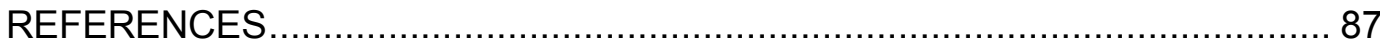

APPENDIX A: CONTACT MODEL PARAMETER ACQUISITION ..................... 94

A.1. Calibration of Instrumentation.................................................. 94

A.2. Error Minimisation ...................................................................... 96 
A.3. Radius of Curvature Measurement ............................................... 99

APPENDIX B: BRUISE DAMAGE CORRELATIONS …............................ 100

APPENDIX C: IMPACT/ABSORBED ENERGY RELATIONS ......................... 105

APPENDIX D: ALGORITHM FLOW CHARTS .......................................... 108

APPENDIX E: DENSITY ACQUISITION .................................................. 111

APPENDIX F: MODEL VALIDATION OUTPUTS ........................................ 112

APPENDIX G: BRUISE DAMAGE SENSITIVITY ...................................... 115

G.1. Sensitivity of the Contact Proximity Radius ................................ 115

G.2. Sensitivity of the Contact Parameters....................................... 119

APPENDIX H: SAMPLE APPLICATIONS ............................................... 120 


\section{LIST OF FIGURES}

Figure 2.1: Transverse section of apple fruit with detailed peel section (Golding \&

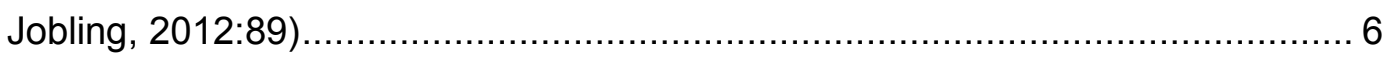

Figure 3.1: A schematic representation of a linear contact model (adapted from

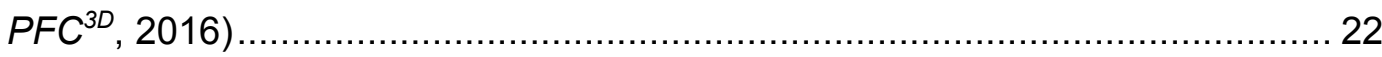

Figure 3.2: Force-deformation curve typical of a quasi-static compression test . 25 Figure 3.3: Compound pendulum experimental set-up for measuring the contact model parameters and the bruise damage ............................................... 26

Figure 3.4: Graphic of the impactors used for the compound pendulum: (a) depicts the spherical impactor, (b) the plate impactor and (c) the apple impactor ......... 27 Figure 3.5: Typical force-time curve recorded during pendulum impact tests.... 28 Figure 3.6: Typical displacement rate curve recorded during pendulum impact tests 28

Figure 3.7: Typical displacement curve recorded during pendulum impact tests 29 Figure 3.8: Typical force-deformation curve resulting from the pendulum impact tests 30

Figure 3.9: Force-deformation curve comparison between the accelerometer and high-speed camera...... 30

Figure 3.10: Comparison between the experimental and contact model forcedeformation curve .............................................................................. 32

Figure 3.11: Comparison between the experimental and contact model force-time curve 32

Figure 3.12: Effect of initial impact velocity on the damping constant for apple-apple

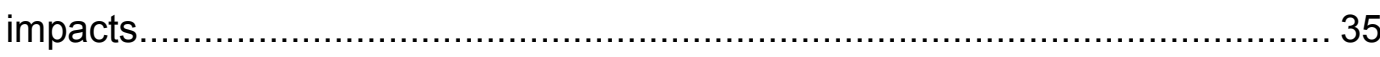

Figure 4.1: Bruise volume measurement .................................................... 37

Figure 4.2: Correlation between peak force and BV for spherical and flat plate

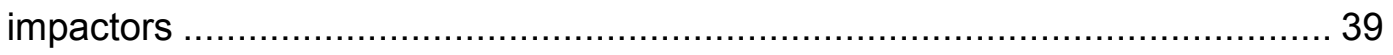

Figure 4.3: Correlation between peak force and BV for apple-apple contact ..... 40 Figure 4.4: BV from two impacts spaced $0-24 \mathrm{~h}$ apart. Error bars indicate $1 \mathrm{SD}$

Figure 4.5: Bruise volume resulting from multiple impacts compared to single

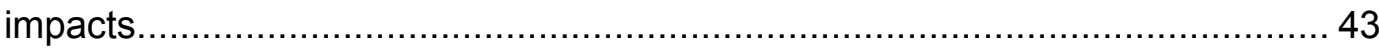

Figure 5.1: Mesh profile of six apples obtained through laser scanning ............ 47 
Figure 5.2: Computational time required with varying clump angle of smoothness

Figure 5.3: Computational time required with an increasing number of clumps in a

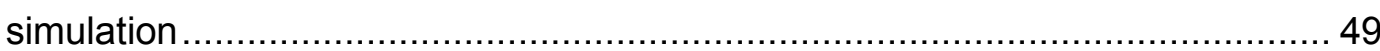

Figure 5.4: Comparison of clumps with differing angles of smoothness $\left[^{\circ}\right]$........ 50 Figure 5.5: Comparison of contact forces (a) with and (b) without single point contact assignment 53

Figure 5.6: Pseudocode outlining the single contact assignment and remove

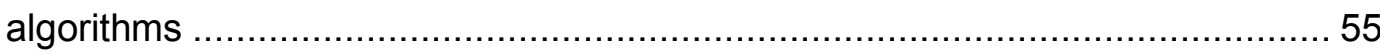

Figure 5.7: Pseudocode outlining the contact data algorithm.......................... 57

Figure 5.8: Pseudocode outlining the bruise overlap algorithm...................... 58

Figure 5.9: Depiction of the facet-wise bruise overlap implementation.............. 59

Figure 5.10: Comparison between the overlap formulation and experimental overlapping bruises for the resulting BV. Errors bars indicate 1 SD for 9 repetitions

60

Figure 6.1: Apple-plate drop test comparing peak forces. Error bars indicate 1 SD for the experiment and $\mathrm{min} / \mathrm{max}$ clump size for the simulation 62 Figure 6.2: Apple-plate drop tests comparing bruise damage, (a) BV, (b) BA and (C) BD. Error bars indicate $1 \mathrm{SD}$ for the experiment and $\mathrm{min} / \mathrm{max}$ clump size for the simulation..... 63

Figure 6.3: Apple-apple drop tests comparing peak force. Error bars indicate 1 SD for the experiments and $\mathrm{min} / \mathrm{max}$ clump size for the simulations 64 Figure 6.4: Apple- apple drop tests comparing bruise damage, (a) BV, (b) BA and (c) BD. Error bars indicate $1 \mathrm{SD}$ for the experiments and min/max clump size for the simulations 65

Figure 6.5: Experimental set-up and DEM model for the vibrating cylinder ........ 66 Figure 6.6: Comparison between the experiment and simulation of the vibrating cylinder with a single apple, (a) BV, (b) BA and (c) BD. Error bars indicate min/max for the experiments and $\mathrm{min} / \mathrm{max}$ clump size for the simulations 67 Figure 6.7: Comparison between the experiment and simulation of the vibrating cylinder with two apples, (a) BV, (b) BA and (c) BD. Error bars indicate min/max for the experiments and $\mathrm{min} / \mathrm{max}$ clump size for the simulations 68 Figure 6.8: Visual comparison between simulation and experiment with the rotating drum set at $200 \mathrm{~mm}$ depth, with spheres on the left, clumps in the centre and the experiment on the right 70 
Figure 6.9: Comparison between simulation and experiment with the rotating drum set at $200 \mathrm{~mm}$ depth, (a) BV, (b) BA and (c) maximum BD respectively. Error bars indicate 1 SD for 5 simulation repetitions 71

Figure 6.10: Comparison between simulation and experiment with the rotating drum set at $400 \mathrm{~mm}$ depth, (a) BV, (b) BA and (c) maximum BD respectively. Error bars indicate 1 SD for 5 simulation repetitions. 73

Figure 6.11: Qualitative comparison between simulation and experiment with structured packing in the vibration box, (a) at the start, (b) after 30 seconds and (c) after 60 seconds.

Figure 6.12: Qualitative comparison between simulation and experiment with random packing in the vibration box, (a) at the start, (b) after 30 seconds and (c)

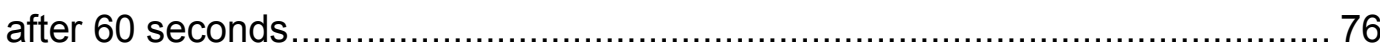
Figure 6.13: Comparison between simulation and experiment for the vibration box with structured packing, (a) BV, (b) BA and (c) maximum BD respectively ........ 77 Figure 6.14: Comparison between simulation and experiment for the vibration box with random packing (a) BV, (b) BA and (c) maximum BD respectively ............ 78 Figure 6.15: Bruise visualisation for the (a) apple drum and (b) vibration cylinder 80

Figure 6.16: Bruise distribution visualisation within the vibration box 81 Figure 6.17: Bruise visualisation comparison between spheres and clumps in the vibration box, (a) experimental apple specimen with visible bruise, (b) clump visualisation in $P F C^{3 D}$, (c) clump visualisation in post-processing, (d) sphere visualisation in post-processing. 82 Figure A.1: Force Calibration Curve 94 Figure A.2: Contour plot of grayscale image of impact test recorded using highspeed camera 95

Figure A.3: Comparison of the impact response between the load cell and impact hammer 96

Figure A.4: Basic outline of the error minimisation algorithm 97 Figure A.5: Combination effect of normal damping constant and normal elastic constant on the sum of residual errors...................................................... 97

Figure A.6: Effect of normal elastic constant on the sum of residual errors....... 98

Figure A.7: Effect of normal damping constant on the sum of residual errors .... 98

Figure A.8: Radius of curvature measurement 99 
Figure B.1: Correlation between peak force and BA for spherical and flat plate

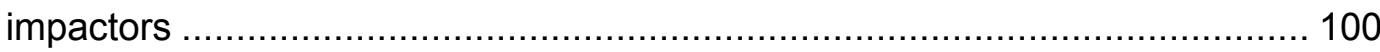

Figure B.2: Correlation between peak force and BD for spherical and flat plate impactors 100

Figure B.3: Correlation between peak force and BA for apple-apple contact ... 101 Figure B.4: Correlation between peak force and BD for apple-apple contact... 101 Figure B.5: Resulting BA from two impacts spaced $0-24 \mathrm{~h}$ apart. Error bars

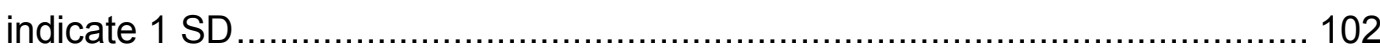
Figure B.6: Resulting BD from two impacts spaced $0-24 \mathrm{~h}$ apart. Error bars indicate $1 \mathrm{SD}$ 102

Figure B.7: Bruise area resulting from multiple impacts compared to single impact profile 103

Figure B.8: Bruise depth resulting from multiple impacts compared to single impact profile 103

Figure B.9: Comparison between the overlap formulation and experimental overlapping bruises for the resulting BA. Error bars indicate 1 SD for 9 repetitions 104

Figure B.10: Comparison between the overlap formulation and experimental overlapping bruises for the resulting BD. Error bars indicate 1 SD for 9 repetitions 104

Figure C.1: Correlation between impact energy and BV for spherical, flat plate and apple impactors 106

Figure C.2: Correlation between impact energy and BA for spherical, flat plate and apple impactors 106

Figure C.3: Correlation between impact energy and BD for spherical, flat plate and apple impactors 106

Figure C.4: Correlation between absorbed energy and BV for spherical, flat plate and apple impactors 107

Figure C.5: Correlation between absorbed energy and BA for spherical, flat plate and apple impactors 107

Figure C.6: Correlation between absorbed energy and BD for spherical, flat plate and apple impactors 107

Figure D.1: Basic algorithm outline for obtaining contact data 108

Figure D.2: Basic algorithm outline for assigning and removing single contacts 109 
Figure D.3: Basic algorithm outline implementing numerical overlap 110

Figure D.4: Resulting BA as visualised on the post-process level

Figure D.5: Basic algorithm outline depicting the bruise visualisation algorithm111

Figure E.1: Density measurement of apples

Figure F.1: Structured packing in the vibration box used for the quantitative comparison between simulation and experiment, (a) at the start, (b) after 30 seconds and (c) after 60 seconds 113

Figure F.2: Visual comparison between simulation and experiment with the rotating drum set at $400 \mathrm{~mm}$ depth. 114

Figure G.1: Sensitivity of the contact proximity radius for the rotating drum set at $200 \mathrm{~mm}$ depth, (a) BV, (b) BA and (c) maximum BD respectively 116

Figure G.2: Comparisons between the mean rotations of balls \& clumps for the vibration box

Figure H.1: Fruit \& vegetable representation in $P F C^{3 D}$ using clumps, (a) models a tomato, (b) models a pomegranate 121

Figure H.2: Fruit \& vegetable representation in $P F C^{3 D}$ using clumps, (a) models a banana, (b) models a pear, (c) models a potato ...................................... 121

Figure H.3: Apple box with packaging material represented in $P F C^{3 D} \ldots \ldots \ldots \ldots 122$

Figure H.4: Representation of an apple packing plant and water fluid conveyor 


\section{LIST OF TABLES}

Table 2.1: Leading apple producing countries for 2005 [tonnes] (Golding \& Jobling,

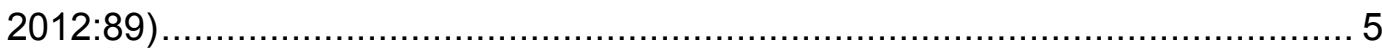

Table 3.1: Normal contact force model parameter values (mean and 1 SD) ...... 33

Table 3.2: Tangential contact force model parameter values (mean and 1 SD). 34

Table 4.1: Bruise damage correlations for the spherical impactor.................... 44

Table 5.1: Resulting contact model properties as specified in $P F C^{3 D} \ldots \ldots \ldots \ldots \ldots . . . . .51$

Table 6.1: Quantitative comparison of the bruise damage of the rotating drum . 74

Table 6.2: Quantitative comparison of the bruise damage of the vibrating box .. 79

Table A.1: Accelerometer calibration information ........................................ 95

Table G.1: Sensitivity of bruise damage for the rotating drum set at $200 \mathrm{~mm}$ depth with regards to the contact proximity radius. Percentage difference is given relative to the experiment.

Table G.2: Comparison between the reduction in number of contacts and bruise damage before and after the overlap formulation 118

Table G.3: Comparison between a $50 \%$ increase/decrease in contact parameter magnitudes 120 


\section{NOMENCLATURE}

\section{Roman Symbols}

c Damping Parameter

$\left[\mathrm{kg} /\left(\mathrm{m}^{1 / 2} \cdot \mathrm{s}\right)\right]$

$c_{n} \quad$ Normal Damping Constant

$\left[\mathrm{kg} /\left(\mathrm{m}^{1 / 2} \cdot \mathrm{s}\right)\right]$

$d_{b} \quad$ Full Depth of Bruise

[mm]

$d_{t} \quad$ Depth from Fruit Surface to Top of Bruise

[mm]

E Elastic Modulus

[Pa]

$E^{*} \quad$ Equivalent Elastic Modulus

[Pa]

$E_{1,2} \quad$ Elastic Moduli of the Two Impacting Bodies

[Pa]

$F_{e} \quad$ Conservative Elastic Part of the Contact Force

[N]

$F_{n} \quad$ Normal Contact Force

[N]

$F_{p} \quad$ Dissipative Plastic Deformation Part of the Contact Force

[N]

$F_{t} \quad$ Tangential Contact Force

[N]

$F_{v} \quad$ Dissipative Viscous Damping Part of the Contact Force

$[\mathrm{N}]$

$G \quad$ Apparent Shear Modulus

$[\mathrm{Pa}]$

$g \quad$ Acceleration Due to Gravity $=9.81$

$\left[\mathrm{m} / \mathrm{s}^{2}\right]$

$G^{*} \quad$ Equivalent Shear Modulus

[Pa]

$G_{1,2} \quad$ Shear Moduli of Impacting Spheres

[Pa]

$k_{n} \quad$ Normal Elastic Constant

$\left[\mathrm{N} / \mathrm{m}^{3 / 2}\right]$

$k_{t} \quad$ Tangential Elastic Constant

$[\mathrm{N} / \mathrm{m}]$

$\varnothing \quad$ Diameter

$[-]$

$R^{*} \quad$ Effective Radius of Curvature

[m]

$R_{1,2} \quad$ Radius of the Impacting Sphere

[m]

$V \quad$ Bruise Volume

$\left[\mathrm{mm}^{3}\right]$

$w_{1,2} \quad$ Bruise Widths Across Major \& Minor Axis

$[\mathrm{mm}]$ 


\section{Greek Symbols}

$\delta_{n} \quad$ Normal Deformation $\quad[\mathrm{m}]$

$\dot{\delta}_{n} \quad$ Normal Deformation Rate $\quad[\mathrm{m} / \mathrm{s}]$

$\delta_{t}$ Tangential Deformation [m]

$\mu_{d} \quad$ Dynamic Friction Coefficient [-]

$v \quad$ Poisson's Ratio [-]

$v \quad$ Effective Poisson's Ratio of Impacting Spheres [-]

$\bar{\omega} \quad$ Mean Particle Rotations (Global Coordinate System) [ $[\mathrm{rad} / \mathrm{s}]$

\section{Abbreviations}

$3 D$

Three-Dimensional

ASAE American Society of Agricultural Engineers

BA Bruise Area

BD Bruise Depth

BV Bruise Volume

DEM Discrete Element Modelling

GD Golden Delicious

PF Peak Force

PR Proximity Radius

$\mathrm{RH} \quad$ Relative Humidity

SD Standard Deviation 
INTRODUCTION

\section{INTRODUCTION}

World trade in fruit account for approximately $12 \%$ of the world's total annual production and the trade in fresh produce is estimated at $70 \times 10^{9}$ US $\$$ annually (Vigneault, Thompson, Wu, Hui, \& LeBlanc, 2009:2). The postharvest handling of this produce does however still introduce mechanical damage and Gonzalez (2009:1) states that 113 million US\$ is lost annually, in the United States alone, due only to apple bruising. Decreasing the amount of postharvest mechanical damage sustained by the fresh produce can consequently lead to large increases in profits for the various fruit cultivators and assist in increasing food security.

One of the most widely cultivated of all fruit is apples. Apples particularly appeal to customers as they have a relatively long postharvest life, have a distinct flavour and their nutritional value is widely known to offer great health benefits. Researchers have determined that the presence of mechanical damage does however accelerate the processes that lead to spoilage and a loss in nutritional value (Opara \& Pathare, 2014:9). As postharvest mechanical damage is present in a large variety of fresh produce it is impractical to investigate the damage mechanisms present in such an extensive range of fruit and vegetables. In addition, a detailed investigation into the damage mechanisms will not be feasible within the scope of this thesis. The investigation will thus be restricted to focusing exclusively on the bruising mechanisms present in apples whilst demonstrating the research process followed in sufficient detail to allow for easy implementation on various other agricultural products susceptible to mechanical damage.

The use of the Discrete Element Method (DEM) is increasingly spreading to uses outside of its original application - particularly the agricultural industry. This is due to the method's versatility as a numerical technique that simulates the dynamic behaviour of particles through collisions (Tijskens, Ramon, \& De Baerdemarker, 2003:494). This allows for the interaction between individual particles to be studied along with the behaviour of a system of particles.

DEM thus provides an excellent means for investigating various stages of the postharvest handling chain responsible for the bruising of apple tissue. DEM also allows for numerical simulation of the various sections of this handing chain and 
INTRODUCTION

can consequently be employed to pre-emptively identify problematic areas. These areas can subsequently be redesigned or eliminated before any physical processes, machines and infrastructure are established which may be shown to introduce bruising damage to the produce.

\subsection{Objectives}

Bruising damage is considered to be the principal form of mechanical damage present during the postharvest handling chain (Van Zeebroeck, Van Linden, Ramon, De Baerdemaeker, Nicolaï \& Tijskens, 2007:158). Impact models that are capable of successfully capturing the behaviour of the apple tissues under loading, impact, and vibration are thus of vital importance in predicting apple bruising.

In order to develop a contact force model that is capable of predicting bruising, the DEM requires two basic contact models namely a normal contact force model and a tangential contact force model. Mohsenin (1986:405) states that the concepts of viscoelasticity are typically applied to study plant and animal tissues. The normal contact force model for viscoelastic materials known as the Kuwabara and Kono (Brilliantov-Pöschel) model is considered by Van Zeebroeck, Tijskens, Van Liedekerke, Deli, De Baerdenaeker and Ramon (2003:472) to be the best representation of the normal behaviour of viscoelastic spheres. Dintwa (2006:49) states that the Mindlin-Deresiewicz tangential contact force model is regarded to be the most accurate, but too complex for the implementation in DEM models involving a large number of particles. Other tangential contact force models are also excluded due to inaccuracies or computational complexity (Dintwa, 2006:49). The Hertz-Mindlin contact force model that approximates the Mindlin-Deresiewicz model will thus be used as it is both accurate and implementable.

As such, the determination of the applicable parameters of the normal and tangential contact force models is a predominant aspect of this study. A bruise prediction model was to be developed and implemented in the DEM environment. The objectives of the study are stipulated below:

1. Determination of the appropriate mechanical properties needed to model the particular apple cultivar under investigation. 
2. Development, design and implementation of an experimental set-up to determine the contact model parameters and quantify bruise damage.

3. The development and implementation of a contact and bruise prediction model in the DEM environment, including bruise overlap.

4. The validation of the developed DEM model through physical and numerical simulation.

\subsection{Motivation}

Current research in the DEM field shows continual movement beyond the original intention of the method for its use in rock mechanics (Cundall \& Strack, 1979). The versatility of the method allows for its application in various scientific and engineering environments. Agricultural applications are of particular interest as a vast amount of the world's production is focused on the cultivation of fresh produce. An overview of DEM applications in the agricultural sector can be found in Tijskens et al. (2003), with more recent studies, applied in the field of fresh produce, being provided by Van Zeebroeck (2005), Dintwa (2006) and Coetzee \& Lombard (2013).

Successfully developing a bruise prediction model in the DEM environment opens up a vast new field of research into the postharvest handling chain of a range of fruit and vegetables. Novel bruise prediction models, applicable to fresh produce that are susceptible to postharvest mechanical damage, can subsequently be developed. Consequently, various phases of the postharvest handling chain can be investigated and refined. The optimisation of produce processing plants can be explored and machinery can be developed that greatly decreases the handling costs of fresh produce.

Stellenbosch University has the required skills, resources and expertise at its disposal to make a significant contribution to this global field. Fruit exports from the Western Cape account for almost $12 \%$ of the value of South Africa's agricultural exports (A growing industry [Agriculture], 2016). In 2008, R 8.5 billion worth of fruit was exported from the province with the majority of this intended for countries within the European Union (A growing industry [Agriculture], 2016). Additionally, auxiliary industries such as fruit canning employ up to 11000 workers (A growing industry [Agriculture], 2016). Some of the largest fruit-packaging companies in the 
world are situated in the Western Cape. Stellenbosch University lies within the heart of this powerful industry and is perfectly situated to develop new world leading engineering technologies within this district.

Achieving the objectives of this study, will allow for the identification of specific areas that are responsible for apple bruising within the apple's journey from tree to market. The developed bruise prediction model can subsequently be used to prevent apple bruising from occurring. This will in turn increase customer satisfaction whilst lowering the product costs as less wastage will occur. Consequently, economic growth and food security will increase in the Western Cape.

\section{LITERATURE REVIEW}

The literature review gives a synopsis of the knowledge areas required for the successful completion of this investigation. This work largely combined the knowledge of the DEM and the agricultural fields. The principles of DEM are accordingly presented, a summary of the knowledge which was required for the study of apples is given and the important aspects of past works are presented.

\subsection{Apples: Background and Bruising}

Golding and Jobling (2012:88) describe apples as being one of agriculture's most successful crops. The apple industry has a long developmental history progressing to the point where apples can be marketed all year round. This is due to the fruit's relatively long postharvest life that is one of its key qualities. Furthermore 1200 different varieties were recognised in 1826 by the Royal Horticultural Society of England (Golding \& Jobling, 2012:88). The astounding range of selections allows for harvesting and marketing dates to be spaced one to two weeks apart greatly extending the availably of apples to customers.

The global investment in the crop is continuing with the world producing close to 60 million tonnes of the fruit in 2005 (Golding \& Jobling, 2012:88). Table 2.1 presents the annual apple production for the main apple producing countries. 
Table 2.1: Leading apple producing countries for 2005 [tonnes] (Golding \& Jobling, 2012:89)

\begin{tabular}{c|ccc|cc}
\multicolumn{3}{c}{ Country } & Apple Production & \multicolumn{1}{c}{ Country } & Apple Production \\
\hline 1 & China & 20406500 & 11 & Chile & 1350000 \\
2 & US & 4474640 & 12 & Argentina & 1262440 \\
3 & Turkey & 2550000 & 13 & Japan & 870000 \\
4 & Iran & 2400000 & 14 & Brazil & 843919 \\
5 & France & 2246351 & 15 & Ukraine & 804000 \\
6 & Italy & 2192000 & 16 & Spain & 797700 \\
7 & Poland & 2050000 & 17 & South Africa & 778630 \\
8 & Russia & 2050000 & 18 & North Korea & 669000 \\
9 & Germany & 1600000 & 19 & Mexico & 580000 \\
10 & India & 1470000 & 20 & Egypt & 550000 \\
& & & & Other & 9646796 \\
& & & & Total & 59591967
\end{tabular}

Table 2.1 indicates that South Africa produces close to eight hundred thousand tonnes of apples per year. Close to seven hundred tonnes are produced in the Western Cape (A growing industry [Agriculture], 2016), which makes the province responsible for approximately $80 \%$ of the country's apple crop.

The apple is a Pome fruit and member of the Rosaceae family (Baugher, 2003:3) with a basic anatomy consisting of a core, flesh, skin, lenticels and cuticle. The anatomy of the apple is illustrated in Figure 2.1.

The core houses the pericarp surrounding the seeds and consists of parenchyma tissue and cartilaginous tissue. At least one seed is commonly located in an ovule and a strong relationship between seed numbers and long term storability has been found. Duly an increase in the amount and viability of seeds through preharvest factors, such as pollination, greatly enhances storability (Golding \& Jobling, 2012:89).

The cortex is the layer of flesh between the core and the skin. In mature fleshly cells, the parenchyma cells have large intercellular airspaces and very thin walls (Golding \& Jobling, 2012:90). These airspaces cause apples to be buoyant in water and are also one of the attributing factors in apple bruising. The skin is comprised of the epidermis and hypodermis and is 4-7 cells deep (Golding \& Jobling, 2012:90). These cells have thickened cell walls and corners to help protect the fruit from damage. 


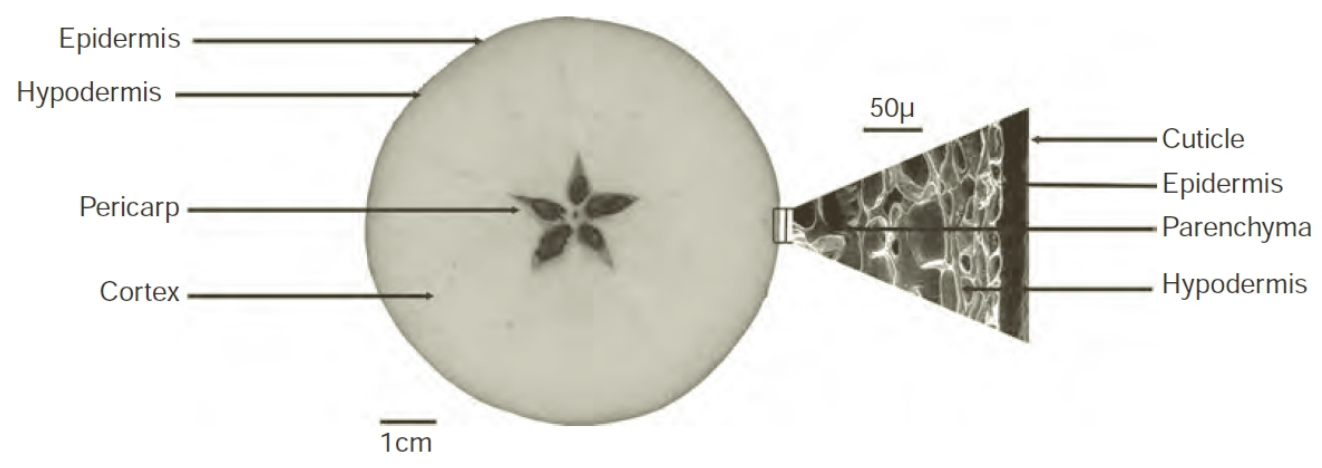

Figure 2.1: Transverse section of apple fruit with detailed peel section (Golding \& Jobling, 2012:89)

The main plant hormone responsible for the ripening of the apples is ethylene with a rapid increase in ethylene production occurring as ripening starts. As such, the control of ethylene is very important in controlling the storage life of apples. Ethylene also controls the softening of apple flesh by regulating the enzymes affecting cell wall degradation (Golding \& Jobling, 2012:91). The enzymes also play a role in bruising of apple flesh.

Preserving fruit quality from orchard to consumer is critical in the control of postharvest pathogens. Thus injury points, such as puncture marks and bruising, must be prevented as they create an access point for infection (Golding \& Jobling, 2012:91). Apple bruising occurs when the cell walls of the apple flesh burst due to impacts. These parenchyma cells are vulnerable as they are surrounded by relatively large intercellular airspaces (Mitsuhashi-Gonzalez, Pitts, Fellman, Curry \& Clary, 2010:509).

The parenchyma cells release various enzymes when bursting and oxidative browning consequently occurs when these enzymes encounter phenolics and the oxygen present within the airspace or from outer air if the skin is ruptured. Mitsuhashi-Gonzalez et al. (2010:510) references various authors who describe bruising as "the consequence of breakage of intercellular bonds, propagation of cell wall ruptures and/or cell deflation as a result of diffusion of cell fluid". It is this destruction of parenchyma cells along with the oxidative browning that consequently occurs that is perceived as bruising of the apple flesh. 
It should be noted that all apples are susceptible to bruising with some light coloured varieties making the perception of bruising more obvious. Apples with a highly blushed peel often conceal the bruised tissue very well. This leads to false preconceptions that these varieties are more resistant to bruising (Golding \& Jobling, 2012:99). Consequently, many researchers make use of the Golden Delicious (hence GD) apple cultivar to investigate apple damage as the bruises are clearly visible and more readily quantifiable. Thus, GD apples were used as the experimental test specimens throughout this study.

\subsection{Postharvest Areas Responsible for Apple Bruising}

Within the agricultural industry product damage from impacts and vibrations may occur during harvesting, transport in bulk bins, sorting, bulk storage, emptying of bulk bins, conveyoring, packaging and packaged transport. Produce with softer tissue may also experience puncture from stems or the environment.

In order to validate and illustrate the capability of the bruise prediction model developed here comparisons with real world scenarios were needed. Therefore, validation experiments were performed that allowed for the accuracy of the contact model application and bruise prediction model to be quantified. Laboratory set-ups that offered representative replications of the potential bruising damage endured during the postharvest handling chain were selected. Subsequently, future investigations into decreasing the bruising damage, which occurs during specific handling stages, can be performed.

Van Zeebroeck, Tijskens, Dintwa, Kafashan, Loodts, De Baerdemaeker and Ramon (2006:94) investigated frequencies experienced by apples during bulk bin transport in orchards. Vibrations below $5 \mathrm{~Hz}$ were found to cause unacceptable bruising damage with a vibration frequency of $4 \mathrm{~Hz}$ identified as critical for mechanical damage. The peak acceleration during the bulk transport was found to be $1.2 \mathrm{~g}$ (Van Zeebroeck et al., 2006:94). Apple arrangements subjected to these vibrations and accelerations can thus be simulated in DEM. Laboratory experiments with similar arrangements, vibrations and frequencies can also be performed. Comparisons between the apple bruising occurring during the 
simulated transport of boxes and the experiments replication of the transport of boxes can thus be made.

When studying mechanical damage during the postharvest handling chain, various researchers investigated fruit stacking heights and arrangements. Some authors suggest that fruit damage due to vibration increases from the bottom to the top of a fruit column (Mohsenin, 1986:560). However, this study was based on peaches and the findings were extrapolated to all fruit. Other researchers reported contradicting results stating that fruit damage increases from the top to the bottom (Fadiji, Coetzee, Chen, Chukwu \& Opara, 2016:117).

Van Zeebroeck et al. (2006:97) investigated the effect of stack height, vibration frequency and acceleration amplitude using DEM. He concluded that bruise damage increases from the top to the bottom layers, but for large unrealistic acceleration amplitudes ( $>1.3 \mathrm{~g}$ ), the opposite occurs. This is attributed to peak acceleration levels increasing and the weight pressure decreasing from the bottom to the top of the column, thus leading to larger contact forces occurring within the top layers. An increase in bruising from the bottom to the top, thus only occurs at acceleration levels that are not realistically experienced under field conditions. A similar study into the distribution of bruising in a fruit column for stack heights, acceleration amplitudes and vibration frequencies can thus be executed once a robust DEM model capable of predicting apple bruising has been developed and validated.

The role of fruit packaging during the handling phases was investigated by Fadiji, Coetzee, Pathare and Opara (2016). The authors reported that irrespective of package design, bruising incidence and susceptibility were highest at the bottom of the package. The successful completion of this study will also allow improved packaging to be designed, optimised and evaluated in the numerical environment before any prototype manufacturing is conducted.

\subsection{Material Properties of Biological Materials}

The mechanical responses of most agricultural products are time dependent and consequently their mechanical behaviour is characterised using the principle of 
viscoelasticity (Morrow \& Mohsenin, 1966:686). The variation and rather unique composition of biological materials naturally make them rather difficult to quantify in terms of mechanical behaviours. Additionally, the mechanical properties of biological materials will change with an increase in maturity level. Restricting the investigation and focusing on a single mature fruit, in this case apples, greatly simplifies the study while allowing for repetition.

Material properties for apple tissue are needed in order to develop contact force models that are capable of accurately representing apple tissue's load deformation behaviour. Primary among the apparent elastic properties are the Young's modulus and Poisson's ratio. The term "apparent properties" is important as biological materials generally violate the fundamental assumptions of homogeneity, isotropy and continuity and a "black-box" type approach is largely used when evaluating these properties (Morrow \& Mohsenin, 1966:688).

Dintwa, Van Zeebroeck, Ramon and Tijskens (2008:270) investigated treatment of apple tissue as a homogeneous substance and varying the elastic properties of the skin and core was shown to have no perceivable effect on the overall material properties. Hence showing that the material properties of the cortex dominate the apple tissue's elastic response.

Force deformation behaviour is typically used to determine the modulus of elasticity for agricultural products (Khodabakhshian \& Emadi, 2011:367). Three theories can be used for the determination of the modulus of elasticity; namely Hooke's law, Hertz's law and Boussinesq's theory. Hooke's law was reported as being inappropriate for agricultural products whereas Boussinesq's theory provides a good approximation when the specimen in question is loaded with a cylindrical plunger (Khodabakhshian \& Emadi, 2011:368).

Researchers most commonly use Hertz's law for the determination of the elastic modulus of agricultural products, as a good agreement between the theoretical and experimental force-deformation curve is achieved (Khodabakhshian \& Emadi, 2011:370). This theory is also less critical and more representative of the loading experienced under practical conditions. Consequently, Hertz's law will be used as a starting point to determine the elastic modulus of apple tissue. Various 
researchers have reported typical values for the elastic modulus $(E)$ of apple tissue ranging from 3 to $5 \mathrm{MPa}$ (Van Zeebroeck, 2005., Dintwa, 2006., Lewis et al., 2008 $\&$ Mohsenin, 1986). These values serve as a good substantiation of the modulus obtained through this study.

The determination of the Poisson's ratio $(v)$ is very difficult with few researches describing detailed investigations into the direct measurement of this ratio for agricultural materials (Morrow \& Mohsenin, 1966:691). Various authors (Van Zeebroeck, 2005., Dintwa, 2006., Lewis et al., 2008 \& Mohsenin, 1986) typically state this value to be in the vicinity of 0.35 . This is considered a good approximation of the materials' transverse elastic response when compressed or stretched. Furthermore, examination of the relationship describing the materials' response under compression reveals $v$ raised to the second power which consequently has a lessened influence on the magnitude of the elastic modulus (Morrow \& Mohsenin, 1978:693).

The dynamic coefficient of friction $\left(\mu_{d}\right)$ of the particles under investigation is also required for the development of a DEM model. The dynamic friction coefficient is used in calculating the tangential contact force experienced by the apples once slip occurs and the static tangential contact force has been overcome. The dynamic friction coefficient is rarely described in literature for fruit-on-fruit or fruit-on-wall contacts. Dintwa, Van Zeebroeck, Tijskens and Ramon (2005:325) investigated the dynamic friction coefficient for apple-on-apple contact and found it to be $0.26 \pm 0.03$. The friction coefficient for apple-on-aluminium contact was also investigated and found as $0.27 \pm 0.08$. These values are in close agreement with those reported in literature namely, Mohsenin (1986:797) who reports apple on galvanised steel contact as 0.28-0.36 for six different apple varieties. Consequently, it is reasonable to set the dynamic friction coefficient for GD apples contacting metal to 0.34 and apple-apple contact to 0.26 for the purposes of this study.

The apparent shear modulus $(G)$ is also needed for the implementation of the DEM model. The shear modulus is required to determine the tangential stiffness within the Hertz-Mindlin contact model used by $P F C^{3 D}$. Direct measurement of the shear modulus involves rheological experiments. The shear modulus can also be 
calculated by utilizing Young's modulus and Poisson's ratio. However, this is only an approximation as the relationship between shear modulus and elastic modulus is only described for isotropic materials - which apples are not.

Van Zeebroeck, Dintwa, Tijskens, Deli, Loodts, De Baerdemaeker and Ramon (2004:116) compared a calculated and experimentally determined shear modulus and found an average difference of $10 \% \pm 8 \%$. Keeping in mind that the Poisson's ratio will vary for individual apples; the use of a calculated shear modulus is adequate for implementation in the DEM model. Van Zeebroeck et al. (2004:117) gave measured values of 1-1.3 MPa for the comparison of the experimentally determined and calculated shear modulus. These serve as a good validation for the values calculated in this investigation.

\subsection{Investigations into the Bruising of Fruit and Vegetables}

As up to $40 \%$ of produce is affected by mechanical damage from harvest to market many researchers have investigated the topic (Opara \& Pathare, 2014:9). One of the most significant contributions to the field is that of Mohsenin (1986) who investigated the material properties needed for the engineering approach for a vast amount of agricultural materials. Biological materials are by their nature varying and consequently several scholars have tried different techniques to correlate bruising and physical parameters. These range from impact force, drop height, impact velocity, impact acceleration and absorbed energy (Opara \& Pathare, 2014:11).

Several researchers have reported a strong linear correlation between bruise volume (hence BV) and absorbed energy. This correlation has resulted in researchers defining a bruise resistance $\left(\mathrm{J} / \mathrm{m}^{3}\right)$ and its inverse bruise susceptibility $\left(\mathrm{m}^{3} / \mathrm{J}\right)$. Numerous other bruise indices quantifying bruise damage in produce have been put forth and are summarised by Opara and Pathare (2014:19). Mohsenin (1986:426) has also described the dynamic yield pressure at which bruising will commence but this only indicates the pressure at which bruising will start to occur and not the extent of the bruising. The use of these bruise quantification indices does not lend themselves to easy implementation in DEM. The relation between peak contact force and BV put forth by Van Zeebroeck (2005:119-144) is much 
better suited for implementation in DEM as impacts between objects of different material properties can be accounted for.

The identification of the areas responsible for mechanical damage to produce within existing postharvest handling chains has led to the development of devices known as instrumented spheres. These devices mimic the response and behaviours of the fruit in question whilst recording the static pressures or dynamic impacts endured by the device. These devices are beneficial for reducing the incidence of bruising on packing lines. However, they are only useful for investigating existing systems within the postharvest handling chain. New designs for packaging, machinery and transport systems must therefore first be prototyped before information of their potential to damage produce can be extracted. Furthermore, multiple repetitions will have to be executed before all the potential damaging areas can be identified. These limitations can be avoided through the use of DEM as areas responsible for bruising damage can be identified within virtual models before any physical prototypes are constructed.

Various methods have also been proposed to measure the BV. The selection of the most appropriate method for the types of impacts the produce receives is important in obtaining accurate BV estimates. A summary of the different methods is presented by Opara and Pathare (2014:13) and include the bruise thickness method, the full depth method, enclosed volume method, unbruised volume removed and the ellipsoid method. All of these methods presume that the bruise has an approximate spherical shape. The bruise thickness method is recommended for small impacts (Opara \& Pathare, 2014:15) and is also simpler to implement. This method is also proposed by Mohsenin (1986:608). The bruise thickness method is illustrated and presented in Chapter 4.

Experimental systems used when investigating the bruising of produce include pendulum impactors, drop testers, compression plates and vibration tables. Pendulum impactors are the most suited for obtaining the viscoelastic data necessary for the development of bruise prediction models in DEM. Pendulum impactors are also the most effective at replicating the typical collision times experienced by the produce. 
Compression plates are incapable of replicating responses that will typically occur during the fruit handling process (Dintwa et al., 2008:274). Drop testers do not offer the same amount of control and reproducibility as pendulum impactors. Vibration tables are capable of mimicking the types of loading and arrangements that produce is likely to experience during handling but offer little in terms of registering the responses of the individual particles.

\subsection{The Discrete Element Method}

The Discrete Element Method was originally developed to simulate the interactions of individual particles and consequently the motion of an assembly of particles within the field of rock mechanics (Tijskens et al., 2003:494). Newton's laws of motion are used to govern the behaviour of every particle within the assembly. Particles are usually spherical in shape but more detailed geometrical representations may be achieved through the use of polygons, implicit surfaces and parametric surfaces (Wachs et al., 2012., Fraige, et al., 2008., Hogue \& Newland, 1994 \& Lu et al., 2012).

Importantly, the use of the term particle within DEM refers to a body occupying a finite amount of space $\left(P F C^{3 D}, 2016\right)$ - unlike the customary definition within mechanics which refers to a body of negligible dimensions. Höhner, Wirtz and Scherer (2013:256) state that several researchers identify particle shape approximation as a difficulty. Therefore, a more detailed discussion on shape approximation is given in Sections 2.5.1 and 2.5.2.

As particles are represented in two or three dimensions (3D) they have rotational degrees of freedom. Additionally the contact forces present between the particles are not conservative in nature due to the presence of frictional forces and the inelastic nature of the collisions (Tijskens et al., 2003:494). In order to apply the appropriate relations governing the resulting forces, DEM requires a contact detection algorithm where particles are approximated as rigid bodies and contacts are modelled as point contacts. As this does not allow the particles to be deformable they are allowed to overlap slightly. This is known as the virtual overlap and the soft particle approach. 
The fundamental building blocks of DEM are the accurate shape representation of the particles, the contact detection algorithm, the contact force models and the time integration. The contact force models govern the interactions of the particles and are a function of the virtual overlap and its rate of change. They are comprised of normal and tangential constituents. Various normal contact force models are described in literature with each having their own advantages and disadvantages.

Depending on the materials being modelled and the objectives of the investigation, the most appropriate model is chosen. Every model fundamentally incorporates an elastic stiffness component and a damping component. The stiffness and damping components in turn depend on the material properties of the particles namely, Young's modulus, Poisson's ratio and the bulk modulus. Normal contact force models describing the behaviour of viscoelastic materials are customarily used to model agricultural produce. Subsequently, they will be explored in this investigation.

Tangential contact force models are employed when oblique collisions occur or when particles are rotating. Accurately describing all the mechanisms involved in tangential contact is very difficult (Zhu \& Yu, 2006:123). Subsequently, only simplified models have been developed in literature. These models have however produced realistic results and proved to be effective when modelling the motion of particles with DEM and are readily employed in commercial software packages.

The simplest tangential contact force model is the Coulomb friction model or linear tangential contact force model. This model only accounts for Coulomb friction and switches from a static friction force to a dynamic friction force once the dynamic regime is entered. Consequently, the dynamic friction coefficient of the particles is required. In the static regime, the static friction force compensates exactly for the external tangential force. The external tangential force is of course unknown so the static friction force is usually determined using a fitted tangential stiffness parameter along with the tangential displacement. In order to avoid the discontinuities present in this model a viscous term may be added (Tijskens et al., 2003:502). This model is the most elementary to implement but does not always accurately capture the tangential behaviour of the particles. 
Various other tangential contact force models are described by Dintwa (2006:49), each offering its own difficulties. The Mindlin-Deresiewicz tangential contact force model is considered to be the most phenomenologically accurate but far too complex to implement in DEM especially for simulations involving a large number of particles. Other models also display problems with accuracy or complexity.

Fortunately, the commercial software code $P F C^{3 D}$ from Itasca Consulting Group, Inc. contains a Hertz-Mindlin Contact force model that approximates the MindlinDeresiewicz model while being much simpler to implement. As this software package is at the disposal of this study, this model was employed as the tangential contact force model for the investigation. For a detailed description of the method, the original theory can be found in Mindlin and Deresiewicz (1953).

Other features of DEM include bonding models that allow particles to adhere to one another and separate when the normal or tangential force exceeds the maximum allowable bonding force. These bonds can also be formed between the particles and the surfaces. Other dedicated models focusing on behaviours such as rolling resistance and creep mechanisms are also available within DEM. As apples and other fruit typically do not adhere to one another, like wet sand for example, bonding and specialist models were thus not required for the purposes of this study.

\subsubsection{Shape Representation Approaches}

Simple shape representations are most commonly used during Discrete Element simulations. The use of circular disks and spheres greatly simplifies the contact detection schemes required and consequently reduces computational complexity. Unfortunately, the representation of the dynamic behaviour of real particles is greatly compromised as spherical particle rotations are only constrained by frictional forces. The rotations of non-spherical shapes are significantly influenced by geometrical interlocking which will also influence the resulting frictional forces (Favier, Abbaspour-Fard, Kremmer, \& Raji, 1999:468).

When studying the bulk behaviour of a system of many granular particles the frictional constant is typically adjusted (subsequently the frictional forces opposing 
the particle's rotations are modified) until a particular simulation's bulk behaviour matches that of a similar physical step-up. Consequently, results, information and conclusions can only be drawn from the system as a whole and not from individual particle-particle interactions. This is referred to as the Bulk Calibration Approach (Coetzee, 2017:123). As this study requires an accurate knowledge of the individual forces between particles simple shape representations cannot be used to represent the particles in question. A highly accurate geometrical representation of the fruit in question is thus required.

Three approaches to achieving relatively accurate geometric representations of particles in DEM are described by Tijskens et al. (2003:497) namely, implicit surfaces, parametric surfaces and polygon models. Implicit surfaces are most often used in DEM as they automatically satisfy the requirement of closed manifolds by their description of a surface with an equation. The simplistic representation of spheres also allows for easy contact detection between pairs. The virtual overlap of the sphere can also easily be determined and it is closely connected to analytical contact force models.

Adversely the use of parametric surfaces for particle shape representation does not always guarantee that they will have closed manifolds. Additionally, contact detection algorithms become more cumbersome than for simple shapes. The use of polygon models to represent surfaces is widely used within the computing environment but their non-smooth nature, relatively complex detection procedure and no guarantee of closed manifolds result in them not being used to represent particles in DEM.

Accordingly, the implicit surface method's description of a sphere through a simple equation allows for straightforward contact detection algorithms to be used and the virtual overlap can easily be related to analytical contact force models. This makes the implicit surfaces description of a sphere the method of choice when representing particles in DEM. Complex particle shapes are thus typically composed of many overlapping spheres in the DEM environment. This rational is also followed by the software package used in this study namely, $P F C^{3 D}$. 


\subsubsection{Shape Representation within $P F C^{3 D}$}

In order to represent surfaces with a complex surface description $P F C^{3 D}$ allows the user to create so-called "clumps". These "clumps" are composed of numerous overlapping spheres referred to as "pebbles" and will behave as rigid solids. These compositions may be specified manually or through the use of the built-in "Bubble Pack" algorithm defined by Taghavi (2011). The "Bubble Pack" method was developed within $P F C^{3 D}$ to allow users to automatically create clumps that approximate arbitrary shapes. The method calculates the mid-surface (the threedimensional extension of the medial axis) of arbitrary objects through Delaunay tetrahedralization. The mid-surface is then used as the locus for the placement of spheres starting with the largest and terminating when the sphere radius falls under a tolerance specified by the user.

This algorithm makes use of a surface description provided by the user and automatically calculates the inertial attributes of the particles required for the DEM simulation. Consequently, a simple geometric file of the particles under investigation can be generated and passed to $P F C^{3 D}$ where an accurate composition of overlapping spheres can be rendered for use within the DEM process.

\subsection{Contact Force Models for Viscoelastic Materials}

The specification of the appropriate contact force model is considered one of the most important elements in DEM (Zhu \& Yu, 2006:122). The contact force model governs the resulting forces that occur when two particles make contact. The contact force model is composed of two constituents, namely a normal and a tangential contact force model.

Various authors made use of mechanical models to represent the viscoelastic behaviour of engineering materials. Agricultural products with a high water content were shown to behave very similarly to the actual mechanical models as the cells behave in a nearly elastic manner whereas the cellular fluids are liquids characterising the viscous component (Morrow \& Mohsenin, 1966:691). Apple flesh, along with other fruit, exhibits this viscoelastic behaviour as well as 
elastic-plastic behaviour above a certain critical impact force (Van Zeebroeck, 2005:36). Models are available which are capable of modelling the impact of elastic-plastic spheres in three phases, namely below yield pressure, above yield pressure and during unloading (Thornton \& Ning, 1997). However, if apple tissue could be modelled as purely viscoelastic, i.e., ignoring the plastic component, the prediction of apple bruising could be somewhat simplified.

Various authors (Van Zeebroeck, 2005., Dintwa, 2006., Lewis et al., 2008. and Mohsenin, 1986) made use of a purely viscoelastic contact force model when investigating apple bruising. This is acceptable as the energy dissipation due to plastic deformation could be accounted for by applying a larger damping parameter $\left(c_{n}\right)$. The increased damping parameter accounted for the energy dissipation occurring due to viscous and plastic deformation.

The accepted normal contact force model for modelling viscoelastic materials is that of Kuwabara and Kono (Dintwa, 2006:62) and is presented by equation 2.1. Dintwa (2006:53) states that through variational calculus analysis from first principles, the equation takes the form shown in 2.2. The elastic constant and the damping constant depend on the material properties of the particles in contact. The elastic constant can be determined directly from Hertz's law but the damping constant is analytically very difficult to determine and is thus customarily established through experimental means.

$$
\begin{aligned}
& F_{n}=k_{n} \delta_{n}{ }^{3 / 2}+ c_{n} \delta_{n}{ }^{1 / 2} \dot{\delta}_{n} \\
& F_{n}=k_{n} \delta_{n}{ }^{3 / 2}+\frac{3}{2} c_{n} k_{n} \delta_{n}{ }^{1 / 2} \dot{\delta}_{n} \\
& \text { with } \quad F_{n}-\text { Normal contact force }[\mathrm{N}] \\
& k_{n}-\text { Normal elastic constant }\left[\mathrm{N} / \mathrm{m}^{3 / 2}\right] \\
& c_{n}-\text { Normal damping constant }\left[\mathrm{kg} /\left(\mathrm{m}^{1 / 2} \cdot \mathrm{s}\right)\right] \\
& \delta_{n}-\text { Normal deformation }[\mathrm{m}] \\
& \dot{\delta}_{n}-\text { Normal deformation rate }[\mathrm{m} / \mathrm{s}]
\end{aligned}
$$

The elastic constant may be obtained directly from Hertz's law as follows: 


$$
\begin{aligned}
k_{n}=\frac{4}{3} \sqrt{R^{*}} E^{*} & \\
\text { with } \quad & E^{*}=\left(\frac{\left(1-v_{1}^{2}\right)}{E_{1}}+\frac{\left(1-v_{2}^{2}\right)}{E_{2}}\right)^{-1}-\text { Equivalent elastic modulus of impacting bodies, } \\
& E_{1} \& E_{2}[\mathrm{~Pa}] \text { are the elastic moduli of the two impacting bodies and } v_{1} \& v_{2} \text { the Poisson's } \\
& \text { ratio of the two bodies } \\
& R^{*}=\left(\frac{1}{R_{1}}+\frac{1}{R_{2}}\right)^{-1}-\text { Effective radius of curvature, } R_{1} \& R_{2} \text { are } \\
& \text { the radii of the impacting bodies [m] }
\end{aligned}
$$

The damping constant $\left(c_{n}\right)$ can be expressed as a mixture of elastic and two viscous constants namely, the steady shear viscosity and the volumetric viscosity (Dintwa et al., 2008:263). The determination of the two viscous constants is however difficult as the volume viscosity is only known for simple Newtonian fluids. Consequently, the damping constant is typically determined experimentally as it must additionally account for energy dissipation due to plastic deformation.

The appropriate tangential contact force model for viscoelastic materials is more challenging. The simple linear contact force model was mentioned in Section 2.5 and has been extended to simulations involving viscoelastic materials by adding a damping constant (Van Zeebroeck, 2005:45). This model does however have weaknesses, as the stiffness and damping constant are only fitted parameters with no physical basis, which compromises the model's accuracy.

As stated in Section 2.5 the Mindlin-Deresiewicz tangential contact force model is the most accurate and was extended by Van Zeebroeck (2005:46) for applications involving viscoelastic materials. However, computational complexity makes this model very difficult to implement and consequently Van Zeebroeck (2005:182) only implemented a linear Coulomb friction tangential contact model.

For the purposes of this investigation, the DEM software's $\left(P F C^{3 D}\right)$ built-in tangential contact force model was used. This model is based on the Mindlin-Deresiewicz tangential model, but is simplified for implementation in $P F C^{3 D}$. The model is presented by equation 2.4 . 


$$
\begin{aligned}
& F_{t}=-\min \left(\left|k_{t} \delta_{t}\right|,\left|\mu_{d} F_{n}\right|\right) \\
& \text { with } \quad F_{t}-\text { Tangential contact force }[\mathrm{N}] \\
& k_{t}-\text { Tangential elastic constant }[\mathrm{N} / \mathrm{m}] \\
& \mu_{d}-\text { Dynamic friction coefficient }[-] \\
& \delta_{t}-\text { Tangential deformation }[\mathrm{m}] \\
& F_{n}-\text { Normal contact force }[\mathrm{N}]
\end{aligned}
$$

Equation 2.4 has the same form as the linear tangential contact model, but the tangential elastic constant is defined in equation 2.5 and varies as the normal contact force varies (PFC $3 D, 2003)$.

$$
\begin{aligned}
& k_{t}=\left(\frac{2(1-v)}{2-v}\right)\left(\frac{3}{2} k_{n}\right)\left|F_{n}\right|^{1 / 3} \\
& \text { with } \\
& \qquad \begin{aligned}
& v=\frac{4 G^{*}-E^{*}}{2 G^{*}-E^{*}}-\text { Effective Poisson's ratio of impacting bodies [-] } \\
& F_{n}-\text { Normal Contact Force [N] } \\
& \qquad G^{*}=\left(\frac{\left(2-v_{1}\right)}{G_{1}}+\frac{\left(2-v_{2}\right)}{G_{2}}\right)^{-1}-G_{1} \& G_{2} \text { is the shear moduli of impacting bodies [Pa] }
\end{aligned}
\end{aligned}
$$

\subsection{Limitations of Previous Studies}

The only significant investigation into the use of DEM for the prediction of apple damage (and other fruit and vegetables in general) is that of Van Zeebroeck (2005). He made use of in-house software named DEMeter++. The use of a commercial code, such as $P F C^{3 D}$, offers several advantages when researching a problem such as the one at hand whilst additionally offering a greater appeal towards the potential commercial applications of such research. The extensive and detailed nature of the study conducted by Van Zeebroeck (2005) thus served as a starting point for this project. Consequently, potential areas of improvement were identified and are discussed further.

The previous study by Van Zeebroeck only made use of simple spheres to represent the apples. According to literature, using better shape representations in general, will lead to an improvement in the modelled dynamics of the particles at both a single particle level and at a bulk level involving a large number of particles (Höhner, Wirtz \& Scherer, 2013:256). Compared to spherical particle, a much improved particle shape representation is possible with the use of clumps (multisphere particles) in $P F C^{3 D}$. 
The study by Van Zeebroeck (2005:182) implemented a simplified tangential contact force model based on the Coulomb friction model. As discussed in Section 2.6 PFC $3{ }^{3 D}$ 's tangential contact force model based on the Mindlin-Deresiewicz model was used. This model is believed to be superior to the simplified model used by Van Zeebroeck (Dintwa, 2006:49).

The lack of a local particle coordinate system in the study by Van Zeebroeck; made it impossible to calculate the BV or bruise area (hence BA) as a result of multiple or overlapping impacts. Van Zeebroeck could calculate the BV, BA and bruise depth (hence BD) for each impact (based on the peak force). However, as soon as multiple impacts occurred in the same position on the apple, or bruises overlapped to some degree, the resultant BV and BA could not be predicted. Thus, Van Zeebroeck could only make use of the maximum BD to compare his results to experimental measurements.

The use of $P F C^{3 D}$ allows for access to a particle local (principal) coordinate system and consequently the effect of multiple overlapping impacts could be accounted for. To this end, an experimental and numerical investigation into the management of multiple overlapping impacts was performed. A post-process algorithm was implemented where more realistic BV's and BA's could be determined from simulation results. Additionally, the type of contact (apple-apple or apple-wall) and the global contact coordinates, could be recorded and analysed during postprocessing. This allowed for bruise damage results to be quantified in terms of $\mathrm{BV}$, $\mathrm{BA}$ and BD, and not only the maximum BD as was the case in Van Zeebroeck's (2005) study.

For the validation experiments performed by Van Zeebroeck (2005:178) the same statistical correlations between peak force and bruise damage were used for impacts involving apple-wall and apple-apple impacts. The correlations for bruise damage were also derived using a spherical metal impactor (Ø50 mm). However, in this study, statistical correlations were obtained for each contact type using a flat impactor as well as an apple-apple impactor. 
CONTACT MODEL AND PARAMETER ACQUIREMENT

\section{CONTACT MODEL AND PARAMETER ACQUIREMENT}

In this chapter, the experimental set-ups and procedures used to obtain the contact model parameter values are presented, together with a detailed discussion of the resultant parameter values.

\subsection{The Contact Model}

Contact force models take the form of spring damper models typically found in engineering vibrational analysis and system modelling. As an example the linear contact model, available in $P F C^{3 D}$, is presented in Figure 3.1. The goal of these models is to use the fundamental kinematical quantities (displacement, velocity and acceleration) to describe the motion of a particle (Inman, 2014:17). Typically, the tangential contact model requires some information from the normal contact model. Accurate modelling of the normal force is thus of crucial importance.

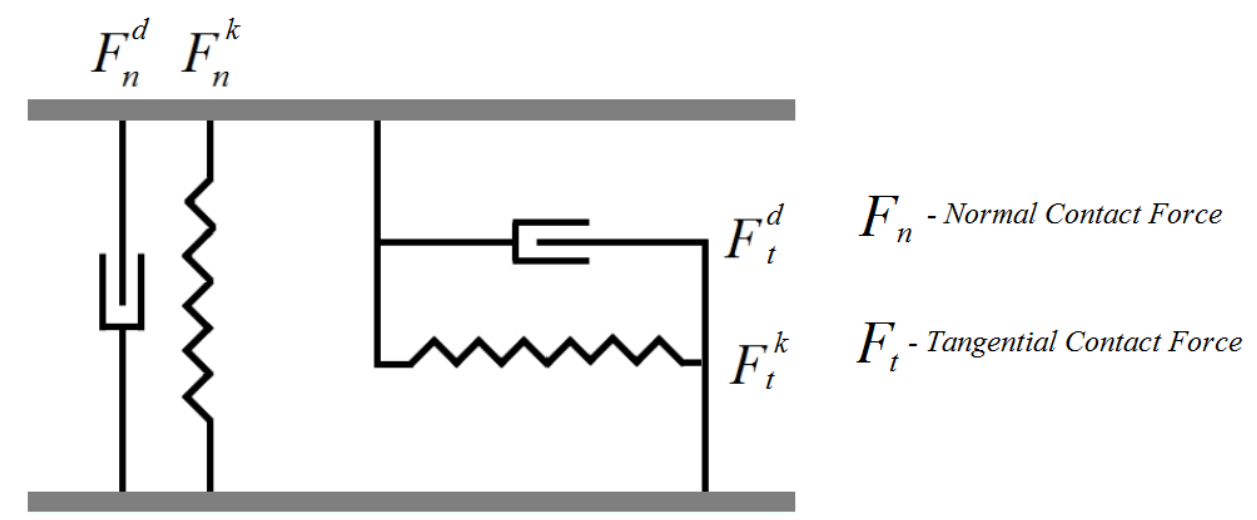

Figure 3.1: A schematic representation of a linear contact model (adapted from $\left.P^{2} C^{3 D}, 2016\right)$

The general form of the force-deformation relationship is presented by Faik and Witteman (2000:5) in equation 3.1. The contact mechanics approach defines three constituents comprising the total force experienced by two particles in contact. The first is the elastic component that accounts for the energy conserved during an impact. Energy lost during an impact is divided between viscous dissipation and plastic deformation. The dissipative viscous damping component is related to the rheological properties of the bodies in contact. The dissipative plastic deformation component is determined from the materials stress-strain curve. 


\section{CONTACT MODEL AND PARAMETER ACQUIREMENT}

$$
\begin{aligned}
F=F_{e}(\delta)+F_{v} & (\delta, \dot{\delta})+F_{p}(\delta, \dot{\delta}) \\
\text { with } \quad & F-\text { Contact force }[\mathrm{N}] \\
& F_{e}-\text { Conservative elastic part of the contact force }[\mathrm{N}] \\
& F_{v}-\text { Dissipative viscous damping part of the contact force }[\mathrm{N}] \\
& F_{p}-\text { Dissipative plastic deformation part of the contact force }[\mathrm{N}] \\
& \delta-\text { Contact deformation }[\mathrm{m}] \\
& \dot{\delta}-\text { Normal deformation rate }[\mathrm{m} / \mathrm{s}]
\end{aligned}
$$

A thorough review of the various normal contact force models is presented by Van Zeebroeck (2005:26-44). Van Zeebroeck's collection and description of the various models is exceedingly useful for anyone who embarks on a DEM investigation where a more detailed description of the contact model is demanded. Nonetheless, the Hertz contact model is presented by equation 3.2 as it is one of the most important relationships in contact mechanics. In the American Society of Agricultural Engineers (ASAE) standards, presented by Mohsenin (1986:825-841), the analytical model shown for determining the modulus of elasticity (Young's modulus) is also based on the Hertz contact theory.

$$
\begin{aligned}
F_{n}=k_{n} \delta_{n}^{3 / 2} & \\
\text { with } & F_{n}-\text { Normal contact force [N] } \\
& k_{n}-\text { Normal elastic constant }\left[\mathrm{N} / \mathrm{m}^{3 / 2}\right] \\
& \delta_{n}-\text { Normal deformation }[\mathrm{m}]
\end{aligned}
$$

Above a certain yield point, in the force-deformation curve, both viscous and plastic energy dissipation will occur for apparent viscoelastic bodies like apples. This "bioyield" point is defined by Mohsenin (1986:135) and describes the loads that apples can withstand without fracture of the cell structure leading to plastic deformation. Ideally, a contact force model that addresses both the viscoelastic and plastic components of the impact is needed. The development of such a model has been addressed by Diels et al. (2016) by combining a viscoelastic and an elastoplastic model, but has as yet not been thoroughly implemented in the DEM environment nor established on a wider scale.

Van Zeebroeck (2005:35) presents two normal contact force models that are most applicable to accurately describe the true impact behaviour of apples. The first of which is referred to as the Kuwabara and Kono model (Kuwabara \& Kono, 1987) 


\section{CONTACT MODEL AND PARAMETER ACQUIREMENT}

where the only energy dissipated is through viscous loss. The second is referred to as the Thornton model (Thornton \& Ning, 1997) where the only energy dissipation mechanism is through plastic deformation. Van Zeebroeck (2005:39) concluded that the Kuwabara and Kono model is best for describing the impact behaviour of apples. This model is in line with spring damper models that have become customary in DEM and treats apples like viscoelastic bodies. Mohsenin (1986:476) showed the viscoelastic nature of fruit and vegetables and stated that agricultural products can in effect be taken to be viscoelastic when analysing them.

The Kuwabara and Kono model mathematically only describes a hysteresis forcedeformation curve and the damping parameter makes no distinction between viscous and plastic energy dissipation. The hysteresis curves typical of fruit and vegetables were also demonstrated by Mohsenin (1986:260). As a result, a higher damping parameter may be used to capture viscous and plastic dissipation - even though this is not physically correct (Van Zeebroeck, 2005:39). The Kuwabara and Kono viscoelastic contact force model is given by equation 2.2 but is repeated for convenience in equation 3.3.

$$
\begin{aligned}
& F_{n}=k_{n} \delta_{n}{ }^{3 / 2}+\frac{3}{2} c_{n} k_{n} \delta_{n}{ }^{1 / 2} \dot{\delta}_{n} \\
& \text { with } \quad F_{n}-\text { Normal contact force }[\mathrm{N}] \\
& k_{n}-\text { Normal elastic constant }\left[\mathrm{N} / \mathrm{m}^{3 / 2}\right] \\
& c_{n}-\text { Normal damping constant }\left[\mathrm{kg} /\left(\mathrm{m}^{1 / 2} \cdot \mathrm{s}\right)\right] \\
& \delta_{n}-\text { Normal deformation }[\mathrm{m}] \\
& \dot{\delta}_{n}-\text { Normal deformation rate }[\mathrm{m} / \mathrm{s}]
\end{aligned}
$$

\subsection{Experimental Set-Up and Procedures}

Obtaining the contact model parameters formed a fundamental component of this project. As stated in Chapters 2.6 and 3.1 a normal elastic constant $\left(k_{n}\right)$ and normal damping constant $\left(c_{n}\right)$ were needed.

\subsubsection{Quasi-Static Compression Tests}




\section{CONTACT MODEL AND PARAMETER ACQUIREMENT}

Determining the elastic constant began with quasi-static compression tests using a texture analyser (Stable Micro Systems, Ltd, TA.XTPlus). These tests slowly $(<1 \mathrm{~mm} / \mathrm{s})$ compressed an apple specimen to produce a force-deformation curve. A typical curve is presented in Figure 3.2 and the general trend is very similar to that found in literature (Mohsenin, 1986:826., Diels et al., 2016:161. \& Holt \& Schoorl, 1983:2021).

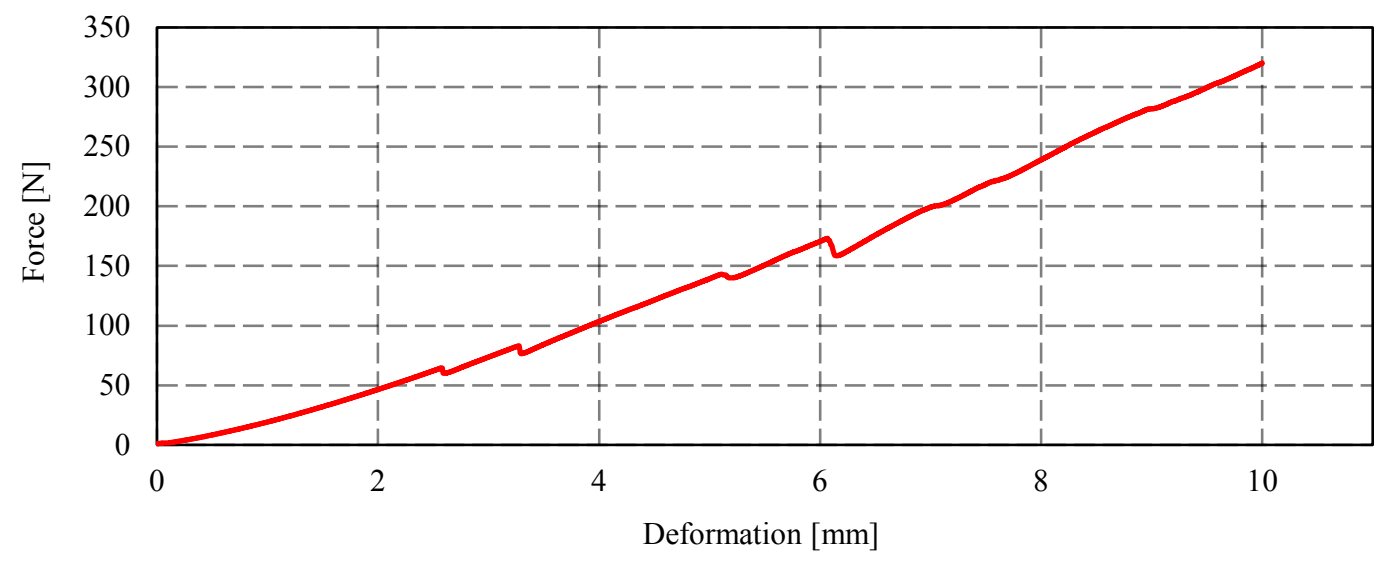

Figure 3.2: Force-deformation curve typical of a quasi-static compression test

From Figure 3.2 the bioyield points are clearly visible where an increase in deformation resulted in a decrease or no change in the force. Furthermore, these compression tests, for food materials of a convex shape, are given by ASAE as the standard for determining the mechanical attributes of food textures, resistance to mechanical injury and force-deformation behaviour. The ASAE method was used to determine the modulus of elasticity for a sample of $20 \mathrm{GD}$ apples and found to be 5.17 ( \pm 0.66$) \mathrm{MPa}$. Once the modulus of elasticity was found and the radius of curvature measured, as described in Appendix $A$, the normal elastic constant $\left(k_{n}\right)$ could theoretically be determined from equation 2.3. Predicting the contact parameters in this way does however assume that the normal deformation rate $\left(\dot{\delta}_{n}\right)$ tends to zero as quasi-static conditions are assumed. Consequently, equation 3.3 reduces to equation 3.2 with dissipative viscous damping not contributing to the total contact force. This leads to a normal elastic constant that is unrealistic for dynamic impacts. This is expected as it is established that the mechanical properties of viscoelastic materials are strain rate dependent (Mohsenin, 1986:523). Accordingly, a compound pendulum was designed for determining the contact model parameters. 
CONTACT MODEL AND PARAMETER ACQUIREMENT

\subsubsection{Pendulum Device}

Many researches have made use of a pendulum device to evaluate the dynamic behaviour of fruit and vegetables during impact (Mohsenin, 1986., Diels et al., 2016., Abedi \& Ahmadi, 2013. \& Opara \& Pathare, 2014). This allows for the material properties of fruit and vegetables to be evaluated under the loading rates and impact conditions that are of interest within the respective investigations. Consequently, an experimental pendulum device was developed as illustrated in Figure 3.3. The use of a compound pendulum allows the location, force/energy magnitude and impact velocities to be controlled with good repeatability. The length of the pendulum's arm was $500 \mathrm{~mm}$. A force transducer was attached to the pendulum tip along with an accelerometer. This allowed for the contact force, displacement rate and displacement, from which the contact model parameters could be derived, to be measured during impact. The specifications and the calibration data for the force transducer (load cell) and accelerometer are detailed in Appendix A.
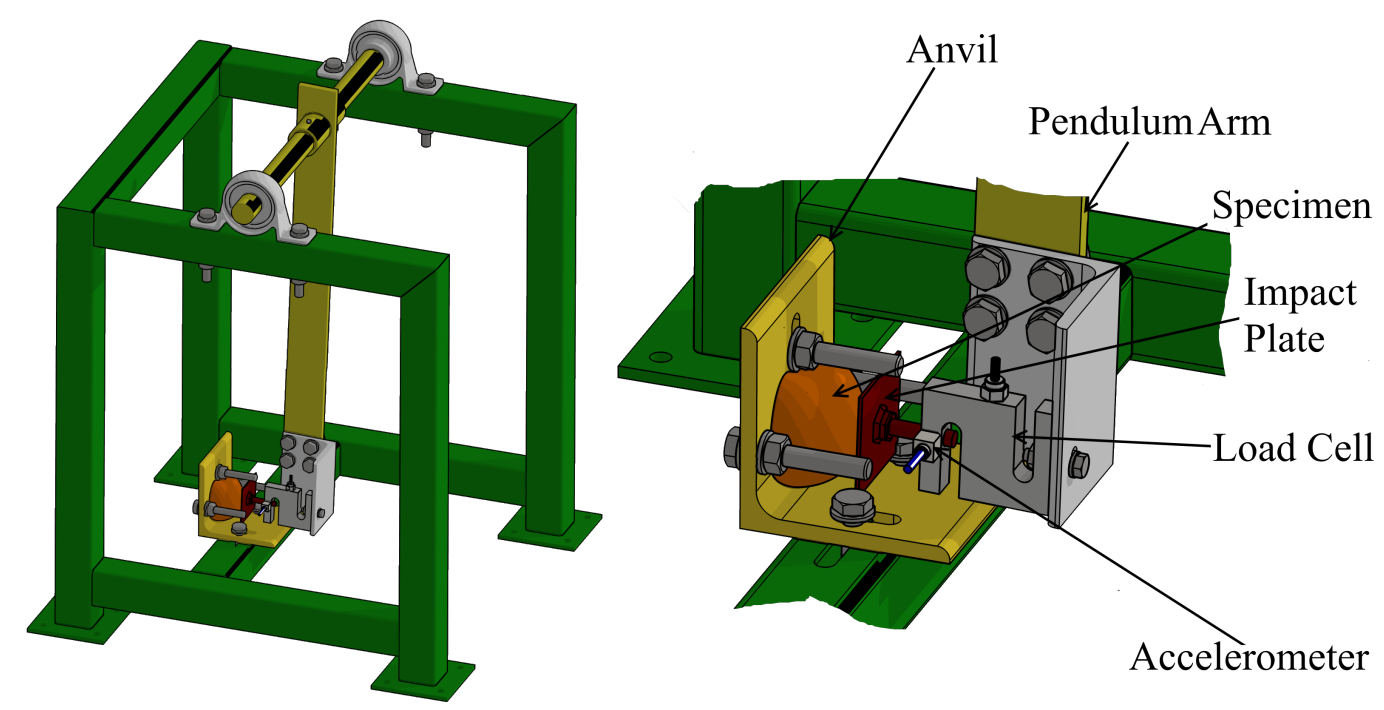

Figure 3.3: Compound pendulum experimental set-up for measuring the contact model parameters and the bruise damage

Three impactors were developed to represent the potential impact conditions that apples are exposed to under realistic conditions. Apples were cut in half, in order to minimise potential deformation between the apple specimen and the anvil, and rigidly strapped to the anvil. The pendulum was rigid enough to minimise any 


\section{CONTACT MODEL AND PARAMETER ACQUIREMENT}

unwarranted vibration and deflection. The three impactors used are illustrated in Figure 3.4.

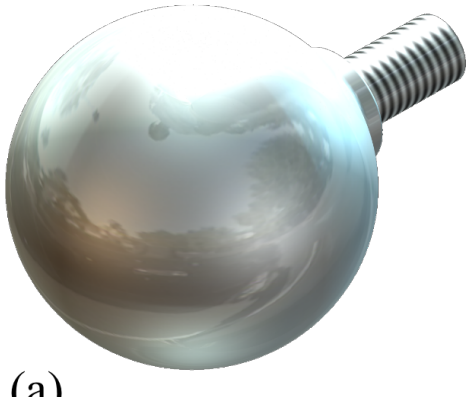

(a)
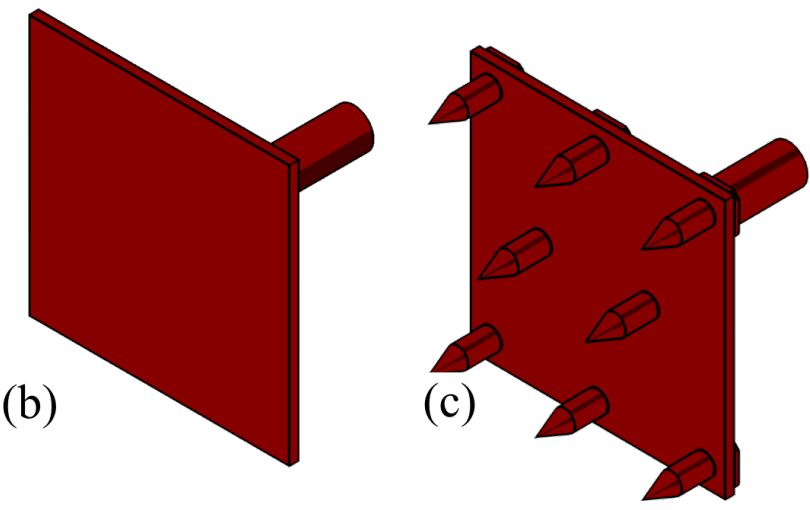

Figure 3.4: Graphic of the impactors used for the compound pendulum: (a) depicts the spherical impactor, (b) the plate impactor and (c) the apple impactor

The first of the impactors was a solid spherical aluminium impactor of $39 \mathrm{~mm}$ in diameter. This allowed very precise bruise measurements to be taken while the impact position could be precisely controlled. Consequently, overlapping impacts and bruise correlations could be effectively investigated. The second impactor (Figure 3.4b) made use of a flat steel plate that was representative of apple-wall contact. The third impactor (Figure 3.4c) employed a spiked steel plate on which half an apple was rigidly mounted. This allowed for apple-apple impacts to be successfully investigated.

Consequently, force-time curves could be recorded for each of the impactors with the sampling frequency of the load cell set at $19200 \mathrm{~Hz}$ using a HBM Quantum X universal amplifier (MX840B) with default Anti-Alias filtering selected (Bessel: $2000 \mathrm{~Hz}$ ). Although the sample rate was quite high, which could lead to elevated noise levels, such a fast sampling rate was required in order to capture the transition point (point of maximum deflection) of the force-deformation curve. Additionally, the accuracy of the load cell and sampling frequency was verified by comparing the impact response of the load cell to an Impulse Force Test Hammer (PCB Piezotronics, MTS Systems Corporation. PCB MD 086C01) with identical response curves measured (refer to Appendix A.1, Figure A.3 for comparison). An example of the force-time curves recorded for the impact tests is given in Figure 3.5 


\section{CONTACT MODEL AND PARAMETER ACQUIREMENT}

using an apple impactor. From this curve, maximum impact force, contact duration and impulse could be determined.

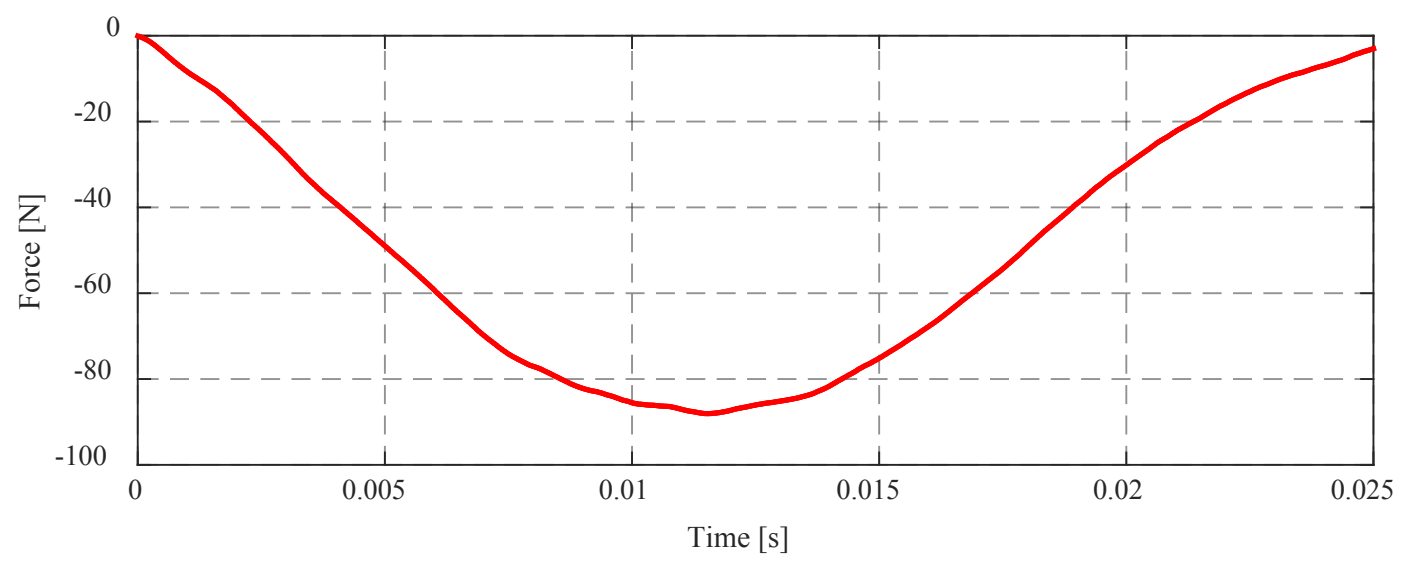

Figure 3.5: Typical force-time curve recorded during pendulum impact tests

The derivation of the contact model parameters also required the measurement of the displacement and the displacement rate. To this end, the accelerometer signal was simultaneously sampled with that of the load cell. Integration and double integration of the accelerometer signal allowed for the displacement rate along with the displacement to be found respectively. An example of the displacement rate curve and displacement curve is given in Figure 3.6 and Figure 3.7 respectively.

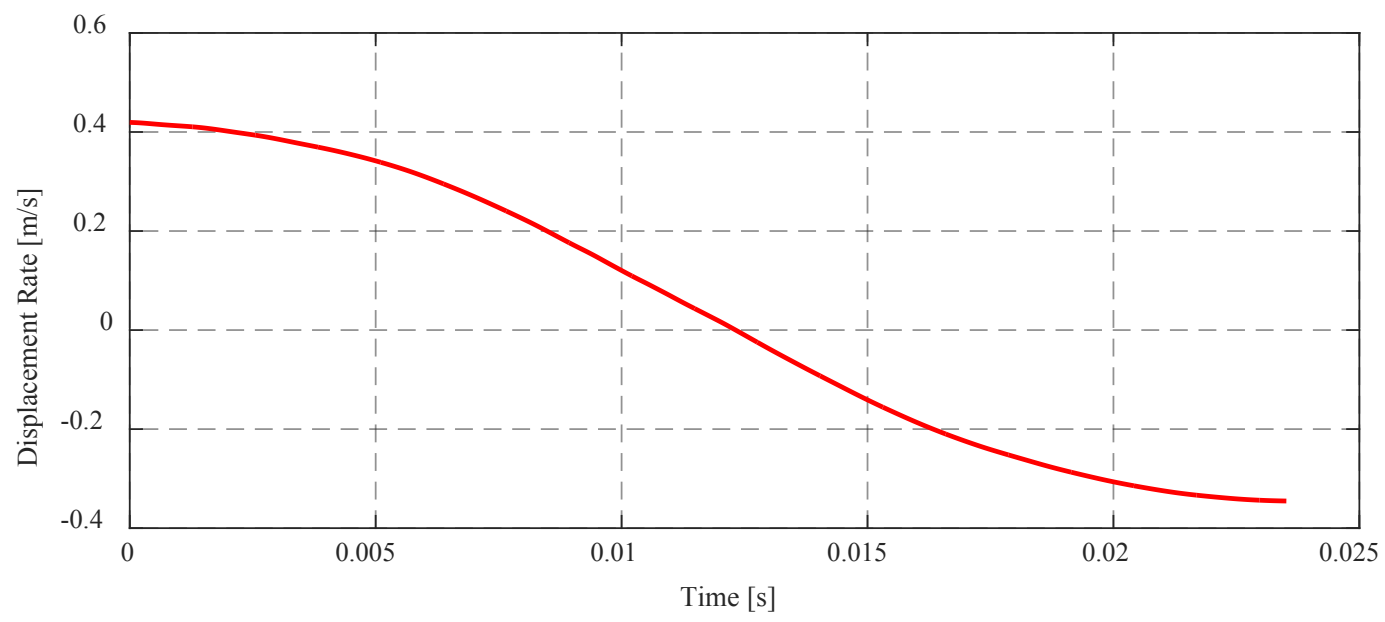

Figure 3.6: Typical displacement rate curve recorded during pendulum impact tests 
CONTACT MODEL AND PARAMETER ACQUIREMENT

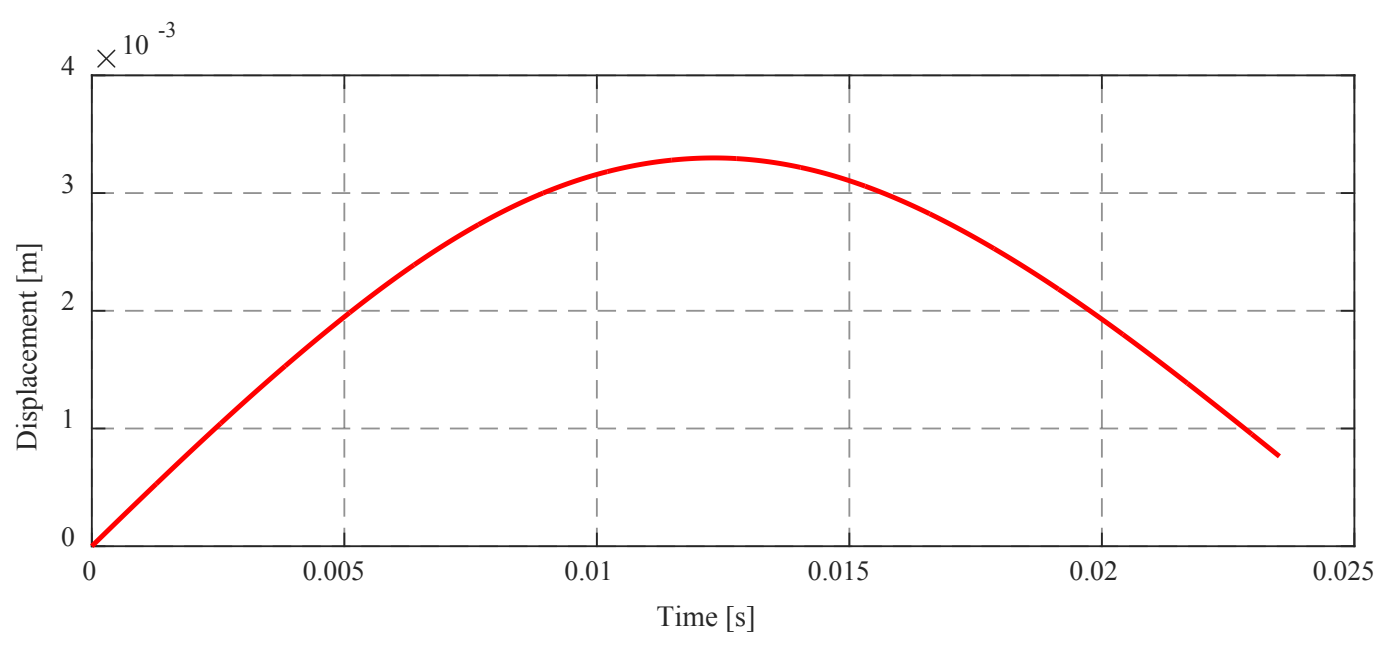

Figure 3.7: Typical displacement curve recorded during pendulum impact tests

Although the use of an accelerometer attached to the pendulum for measuring the displacement and displacement rate, has been described in literature, there are inaccuracies that may be present from such numerical integration schemes (Van Zeebroeck et al, 2003:471). The inaccuracies occur due to the uncertainty of the integration constants and the integration of low-frequency noise. However, accurate measurements are feasible for short time intervals (like impacts) while integration of longer signals ( $>10 \mathrm{~s}$ ) is rather unsuccessful (Abir, J., Longo, S., Morantz, P \& Shore, P. 2016:2). Considering the short time durations during the experiments $(<0.035 \mathrm{~s})$ the use of numerical integration was feasible. The signals were sampled and integrated with the amplifier's (HBM Quantum X) built-in integration function. Combining a force-time curve and a displacement-time curve, a force-deformation curve could be produced with a typical example given in Figure 3.8.

The integration of the accelerometer signal was further verified with the use of a high-speed camera (Olympus, i-SPEED 3 TR). Five tests were performed where the impact between the spherical impactor and the specimen was recorded at 3000 frames per second. Subsequently, an algorithm was programmed in MATLAB (2015) which tracked the pixels of a coloured dot on the spherical impactor over the total duration of impact (a depiction of the method is given in Figure A.2 in Appendix A). Thus, a displacement-time curve could be constructed from the images. This curve could then be synchronised with the force-time curve recorded 


\section{CONTACT MODEL AND PARAMETER ACQUIREMENT}

by the load cell. The force-deformation curve resulting from the high-speed analysis could be compared to the force-deformation curve obtained for the same impact with the use of the accelerometer. The comparison between a typical highspeed and accelerometer force-deformation curve is presented in Figure 3.9.

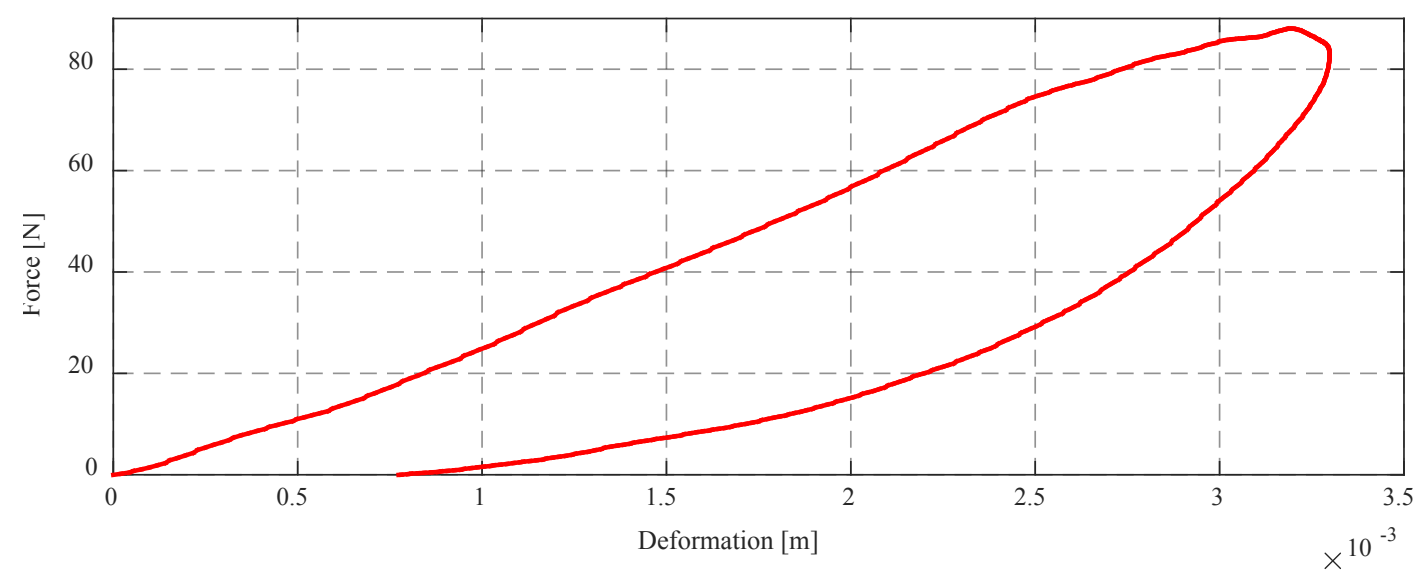

Figure 3.8: Typical force-deformation curve resulting from the pendulum impact tests

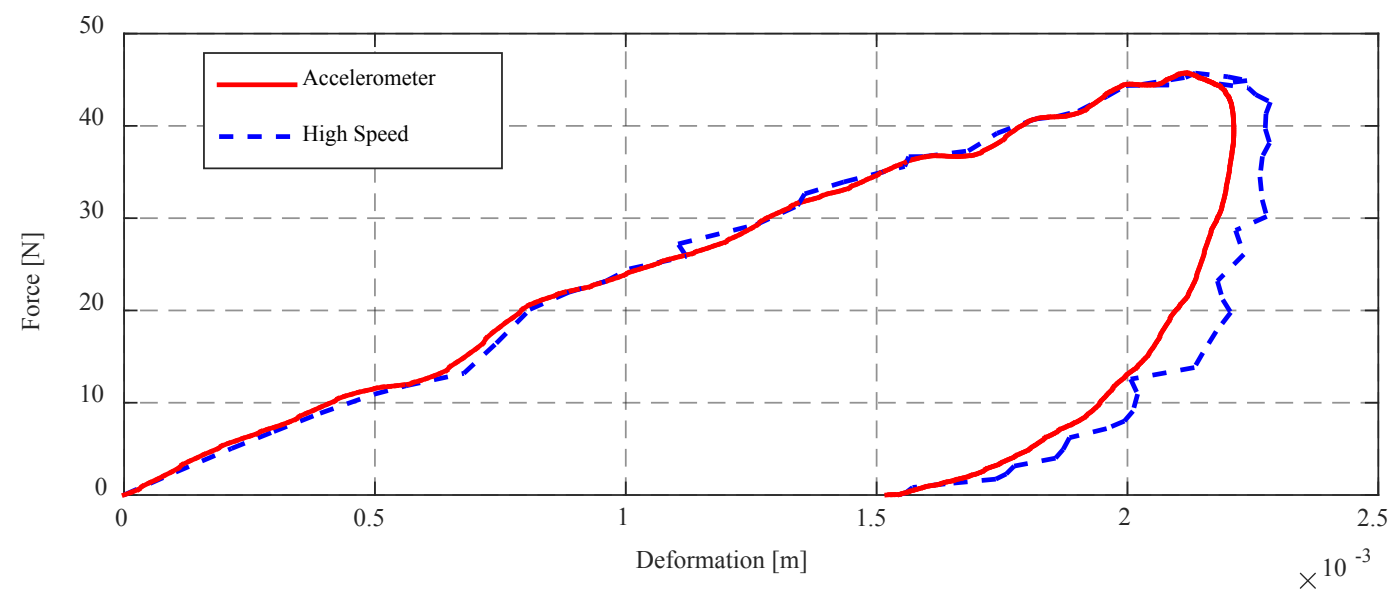

Figure 3.9: Force-deformation curve comparison between the accelerometer and high-speed camera

From Figure 3.9, an excellent agreement is observed between the high-speed and accelerometer force-deformation curves. No significant difference between the initial impact velocity or total deformation was found (correlation coefficients ranged from 0.83 to 0.97 between the high-speed camera and accelerometer for the deformation-time curves). When comparing Figure 3.8 and Figure 3.9 it should be noted that two different tests are given, Figure 3.8 experienced a larger peak 


\section{CONTACT MODEL AND PARAMETER ACQUIREMENT}

force and made use of the apple-apple impactor. Figure 3.9 made use of the spherical impactor and had a smaller peak force which produced a curve that is less smooth than that of Figure 3.8. Hence, the use of the accelerometer to measure the deformation and deformation rate was deemed suitable for the purposes of this study.

\subsection{Parameter Fitting}

Once force-deformation curves for the various impact conditions were obtained, their respective contact model parameters were acquired. To this end, a minimisation algorithm was developed which minimised the sum of the squares of the residuals between the measured data and the estimated values obtained from equation 3.3. Similar methods were used in literature (Van Zeebroeck, 2005 \& Horabik et al., 2017). The values measured for the deformation $\left(\delta_{n}\right)$ and deformation rate $\left(\dot{\delta}_{n}\right)$ were utilised together with estimated search boundaries for the normal damping constant $\left(c_{n}\right)$ and the normal elastic constant $\left(k_{n}\right)$ of equation 3.3. The sum of the squares of the residuals between the resulting normal contact force $\left(F_{n}\right)$ and the measured impact force was calculated. An overview of the algorithm along with depictions of the error minimisation is presented in Appendix A.

For each impact test the optimal combination of $k_{n}$ and $c_{n}$ was found, along with a correlation coefficient, which resulted in the best fit between the experimental data and the Kuwabara and Kono viscoelastic contact force model (equation 3.3). An example of the resulting contact model curve matched to the experimental data is presented in Figure 3.10 for the force-deformation curve and Figure 3.11 for the force-time curve. A good correlation between the experimental data and contact model was found with the correlation coefficient $\left(R^{2}\right)$, for all tests conducted ranging from 0.82 to 0.99 .

Each of the impact conditions made use of 30 test specimens (each impacted once) with the use of the impactors illustrated in Figure 3.4 for a total of 90 tests. Importantly the bruise prediction models, described in Chapter 4, were derived from the same impact test data used for the contact model parameter acquirement. This served to reduce the inherent biological variability of the samples as the same 


\section{CONTACT MODEL AND PARAMETER ACQUIREMENT}

test data used to derive the contact model parameters was used to derive the bruise correlations. The impact force ranged from $20 \mathrm{~N}$ to $160 \mathrm{~N}$ along with the initial impact velocity from 0.3 to $1.0 \mathrm{~m} / \mathrm{s}$.

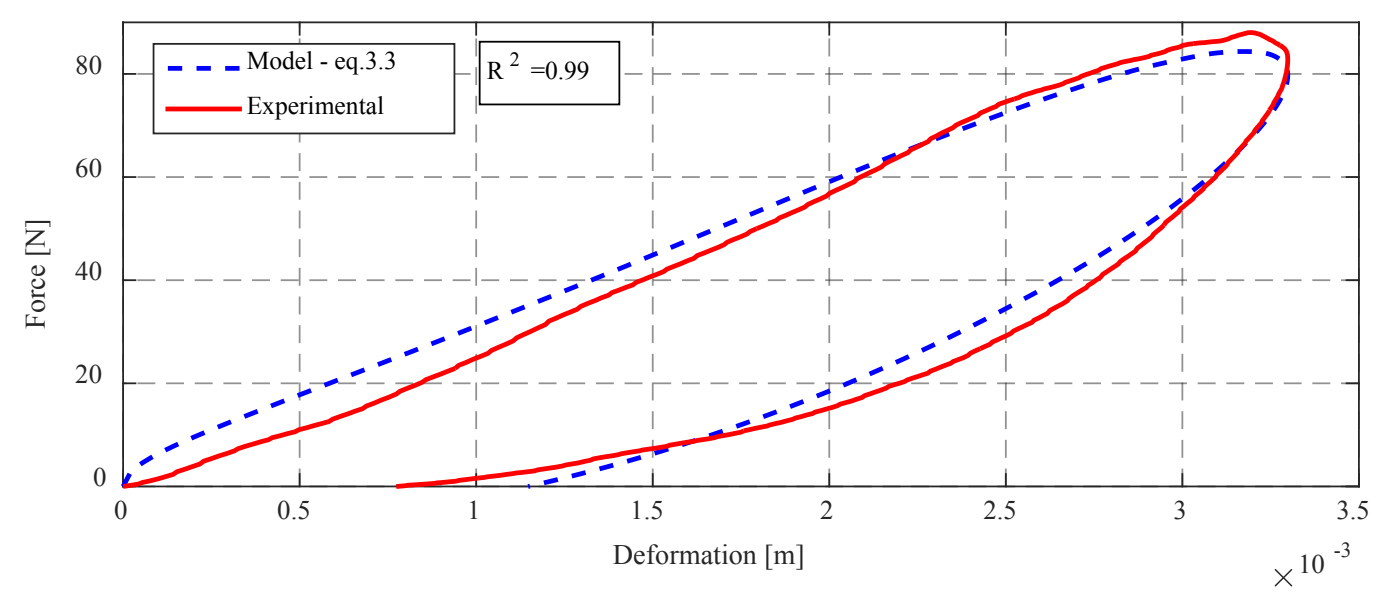

Figure 3.10: Comparison between the experimental and contact model forcedeformation curve

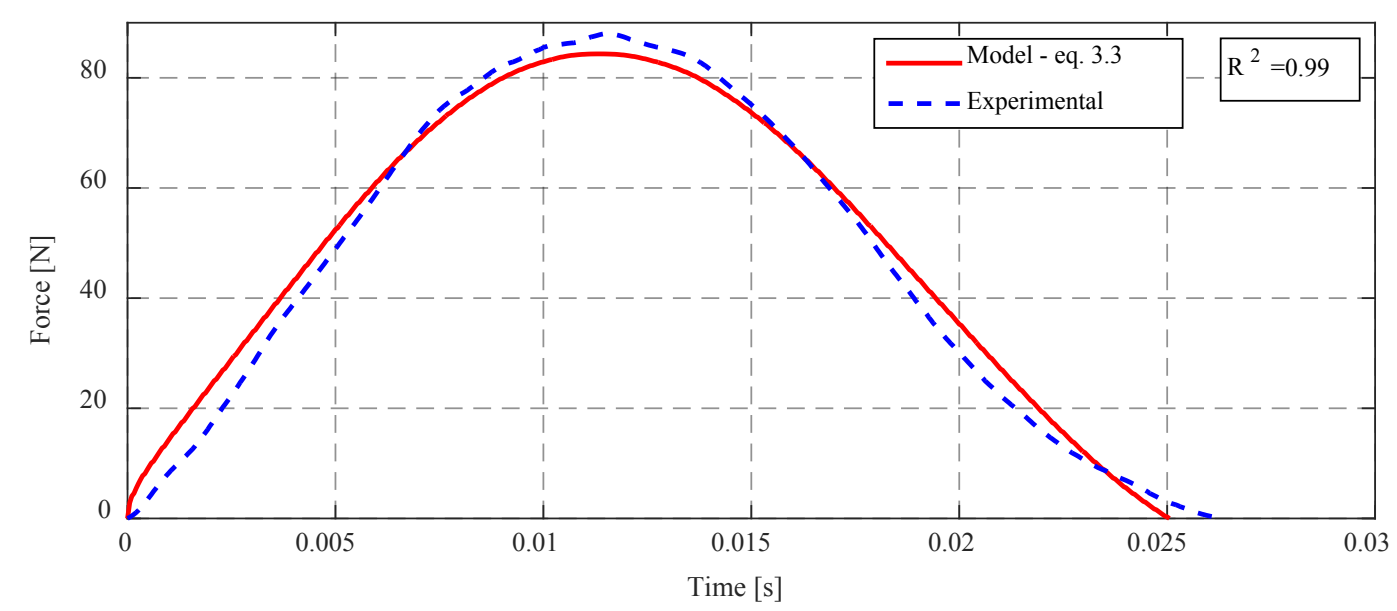

Figure 3.11: Comparison between the experimental and contact model force-time curve

The mean normal elastic constant $\left(k_{n}\right)$ and normal damping constant $\left(c_{n}\right)$ for each of the contact situations were subsequently found. Each test sample's equivalent elastic modulus $\left(E^{*}\right)$ was calculated with the use of the sample's effective radius of curvature as shown in equation 2.3. Additionally, the (apparent) elastic modulus 


\section{CONTACT MODEL AND PARAMETER ACQUIREMENT}

of the apples could be calculated for the two contacting situations with the use of each impacting body's Poisson's ratio. The Poisson's ratio for apples was taken equal to 0.35 as described in Chapter 2.3.

\subsection{Apples Used}

For the determination of the optimal combination of $k_{n}$ and $c_{n}$ and bruise prediction models for the apple-wall and apple-apple impacting conditions; 500 'Golden Delicious' apples were obtained from the Betko Fresh Produce packhouse, Villiersdorp, Western Cape, South Africa (335 59' 46.2408" S, $19^{\circ} 17^{\prime} 2.8248 "$ E). Apples were picked in March 2017 and stored for \pm 4 months in cold storage at \pm $1{ }^{\circ} \mathrm{C}, 93 \%$ relative humidity (hence $\mathrm{RH}$ ). Apples were transported to the test laboratory and were stored at the optimal conditions $\left(3^{\circ} \mathrm{C}, 85 \% \mathrm{RH}\right)$ as stated by Van Zeebroeck, Van Linden, Darius, De Ketelaere, Ramon and Tijskens (2007:11). All the test specimen's radii of curvature were measured as described in Appendix A.

\subsection{Results}

The resulting contact model parameters for the normal contact force are presented in Table 3.1 along with the standard deviation (hence SD) of the parameters.

Table 3.1: Normal contact force model parameter values (mean and $1 \mathrm{SD}$ )

\begin{tabular}{c|cccc} 
Description & Symbol & Unit & Apple-Wall & Apple-Apple \\
\hline $\begin{array}{c}\text { Mean Elastic } \\
\text { Constant }\end{array}$ & $\left(\overline{k_{n}}\right) \pm \sigma_{k_{n}}$ & {$\left[\mathrm{kN} / \mathrm{m}^{3} / 2\right]$} & $675 \pm 236$ & $365 \pm 143$ \\
$\begin{array}{c}\text { Mean Effective } \\
\text { Damping } \\
\text { Constant }\end{array}$ & $\left(\overline{c_{n}^{*}}\right) \pm \sigma_{c_{n}}$ & {$\left[\mathrm{~kg} /\left(\mathrm{m}^{1 / 2} \cdot \mathrm{s}\right)\right]$} & $3.51 \pm 1.69$ & $3.50 \pm 2.08$ \\
$\begin{array}{c}\text { Mean Equivalent } \\
\text { Elastic Modulus }\end{array}$ & $\left(\overline{E^{*}}\right) \pm \sigma_{E^{*}}$ & $\mathrm{MPa}$ & $2.87 \pm 0.99$ & $2.18 \pm 0.87$ \\
$\begin{array}{c}\text { Mean Apparent } \\
\text { Elastic Modulus }\end{array}$ & $\left(\bar{E}_{\text {apple* }}\right) \pm \sigma_{E_{\text {apple }}}$ & $\mathrm{MPa}$ & $2.52 \pm 0.87$ & $3.83 \pm 1.52$
\end{tabular}

In addition to the acquirement of the appropriate normal contact force parameters the tangential contact force model also required the attainment of the shear moduli 


\section{CONTACT MODEL AND PARAMETER ACQUIREMENT}

of the impacting bodies. As stated in Chapter 2.5 PFC ${ }^{3 D}$ 's built-in Mindlin-Deresiewicz tangential contact force model was used. Consequently, the mean elastic modulus of the apples, for each impacting case, was used to determine the (apparent) shear modulus of the apples. The shear modulus was determined using equation 3.4 .

$$
G=\frac{E}{2(1+\nu)}
$$

The use of the equation 3.4 only connects the elastic modulus to the shear modulus for isotropic materials, but as discussed in Chapter 2.3, Dintwa et al. (2008:270) demonstrated that the cortex governs the apple tissue's elastic response. Accordingly, the use of equation 3.4 would permit an accurate indication of the apple tissue's shear modulus for a given contact situation. The effective Poisson's ratio of the impacting bodies was calculated as described in $P F C^{3 D}$ 's Mindlin-Deresiewicz tangential contact formulation as shown in equation 2.5. The resulting mean effective shear modulus, shear modulus of the apples and effective Poisson's ratio for the derived contact model are presented in Table 3.2:

Table 3.2: Tangential contact force model parameter values (mean and 1 SD)

\begin{tabular}{l|cccc}
\multicolumn{1}{c}{ Description } & Symbol & Unit & Apple-Wall & Apple-Apple \\
\hline $\begin{array}{c}\text { Mean Equivalent } \\
\text { Shear Modulus }\end{array}$ & $\left(\overline{G^{*}}\right) \pm \sigma_{G^{*}}$ & $\mathrm{MPa}$ & $0.565 \pm 0.195$ & $0.429 \pm 0.171$ \\
Mean Apparent \\
$\begin{array}{l}\text { Shear Modulus } \\
\text { Mean Effective } \\
\text { Poisson's Ratio }\end{array}$ & $\left(\overline{G_{\text {apple }}}\right) \pm \sigma_{G_{\text {apple }}}$ & $\mathrm{MPa}$ & $0.902 \pm 0.36$ & $1.42 \pm 0.57$ \\
& $(\bar{v}) \pm \sigma_{v}$ & & & \\
& & {$[-]$} & 0.35 & 0.35
\end{tabular}

The validity of the use of the mean contact model parameters summarized in Table 3.1 and Table 3.2 was verified in Chapter 6.2 with the use of drop tests. An excellent correlation between the experimental and predicted impact force for the apple-wall contact was observed, as shown in Figure 6.1. The apple-apple contact did however indicate the sensitivity of the effective normal damping constant for the apple-apple contact case. Consequently, the test data for the apple-apple contact's normal damping constant was re-examined as a correlation between the 


\section{CONTACT MODEL AND PARAMETER ACQUIREMENT}

damping parameter and initial impact velocity had been described in literature (Lankarani \& Nikravesh, 1990.371).

A correlation between initial impact velocity and the damping constant was found (no such correlation was observed for the apple-wall case). The sensitivity of the apple-apple contact to impact velocity can be attributed to the higher degree of viscous dissipation and plastic deformation that is present when two apples impact as opposed to only one apple in the apple-wall case. For the drop tests described in Chapter 6.2, the damping constant for the apple-apple tests was consequently programmed as a linear function to the initial velocity, as observed in Figure 3.12, in the range of $0-1 \mathrm{~m} / \mathrm{s}$.

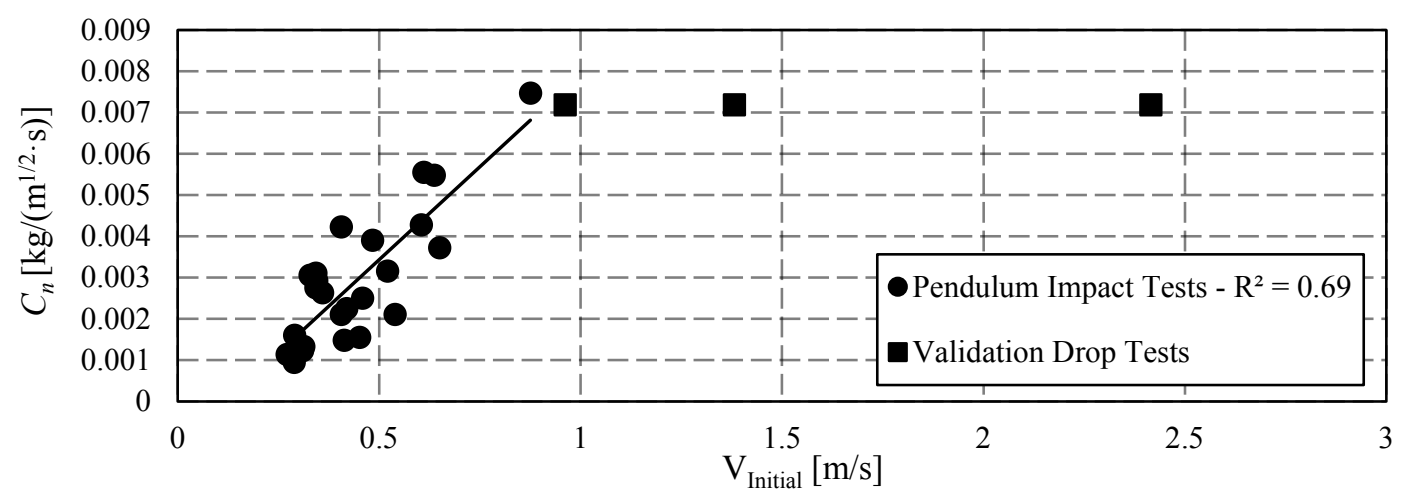

Figure 3.12: Effect of initial impact velocity on the damping constant for appleapple impacts

For the larger initial impact velocities $>1 \mathrm{~m} / \mathrm{s}$ (tested within the drop tests in Chapter 6.2) an upper limit was placed on the damping constant corresponding to the maximum derived damping constant for the apple-apple contact case, namely, $7.18 \times 10^{-3}$. Subsequently, the verification drop tests were repeated for the appleapple case with an excellent correlation as shown in Figure 6.3. The resulting damping constant used in the contact model for the apple-apple case is described by equation 3.5 .

$$
\begin{gathered}
C_{n}{ }^{*}=\left\{\begin{array}{cc}
0 & \text { for } V_{i} \leq 0.13 \mathrm{~m} / \mathrm{s} \\
0.009 V_{i}-0.0011 & \text { for } 0.13<V_{i}<0.92 \mathrm{~m} / \mathrm{s} \\
7.18 \times 10^{-3} & \text { for } V_{i} \geq 0.92 \mathrm{~m} / \mathrm{s}
\end{array}\right. \\
\text { with } \quad C_{n}{ }^{*}-\text { Effective normal damping constant }\left[\mathrm{kg} /\left(\mathrm{m}^{1 / 2} \cdot \mathrm{s}\right)\right]
\end{gathered}
$$




\subsection{Discussion}

The contact parameters for the implementation of the Kuwabara and Kono viscoelastic contact force model and Mindlin-Deresiewicz tangential contact force model, representing the force-deformation behaviour of apples were successfully determined. The elastic modulus of apples under realistic dynamic conditions was lower than the elastic modulus found with the ASAE proposed method for determining the modulus of elasticity for food materials of a convex shape under quasi-static conditions. The ASAE method resulted in an elastic modulus of 5.17 $( \pm 0.66) \mathrm{MPa}$ and the elastic moduli derived from the pendulum tests gave 2.52 ( \pm $0.87) \mathrm{MPa}$ for apple-wall and $3.82( \pm 1.57) \mathrm{MPa}$ for apple-apple contacts. Although the pendulum test resulted in elastic moduli that were lower than that of the quasistatic tests, the order of magnitude of the elastic moduli was comparable. Furthermore, the values were in the same range as those reported in literature with Mohsenin (1986:793) describing elastic moduli of 3.8 to $7.0 \mathrm{MPa}$ for GD apples and Lewis, Yoxall, Marshall, and Canty, (2008:39) reporting 4.0 MPa. The relatively high standard deviations were expected due to the inherent biological variability of the material in question. No relationship between the elastic modulus and the initial impact velocity could be found. A fair correlation between initial impact velocity and normal damping constant for apple-apple contacts was observed. The forces resulting from the contact model parameters were validated and are presented in Chapter 6.2. The derivation of the contact model parameters, using a nonlinear least squares error minimisation algorithm and a pendulum impactor, was shown to be suitable for the representation of apple tissue's response during impact.

\section{BRUISE PREDICTION MODEL}

In order to predict the bruise damage, a relationship between the impact force (predicted by the contact force model) and subsequent bruising was needed. This related the impact characteristics and the fruit properties to the bruising damage.

\subsection{Quantification of Bruising}

Numerous investigations into apple bruising have been performed and are named in Chapter 2.4. These investigators have measured absorbed energy, force, impact 
velocity, acceleration and drop height when studying bruising damage. Comparing and interpreting data from different studies is however difficult resulting in them being of little use to apple traders when trying to reduce bruise losses (Lewis et al., 2008:37). Equipping DEM software with the capability of quantifying bruise damage will result in forthright identification of ways to reduce bruise losses. Relating bruise damage and peak contact force is the most suited for implementation in DEM as impacts between objects of different material properties can be accounted for (Van Zeebroeck et al., 2007:16).

Fruit quality is typically judged by appearance with a bruise threshold of $100 \mathrm{~mm}^{2}$ used in industry for determining when apples should be discarded (Lewis et al., 2008:37). This has led to results being quantified in terms of BA rather than BV. However, recording bruise severity is rather subjective and has made it difficult to compare results. Many researchers report a strong linear correlation between BV and impact energy (Pang et al., 1992., Schoorl \& Holt, 1980., Opara \& Pathare, 2014., Lewis et al., 2008. and Mohsenin, 1986). Bruise volume measurement additionally gives a better idea of the total impact damage as BV can vary independently of bruise surface area (Pang, Studman \& Ward, 1992:233). Calculation of the BV was made using the bruise thickness method presented by Opara and Pathare (2014:13) and provides the best estimates for small impacts, equation 4.1. An illustration of the measurement technique is given in Figure 4.1.

$$
\begin{aligned}
& V=\frac{\pi\left(d_{b}-d_{t}\right)}{24}\left(3 w_{1} w_{2}+4\left(d_{b}-d_{t}\right)^{2}\right) \\
& \text { with } \quad \begin{array}{l}
V-\text { Bruise volume }\left[\mathrm{mm}^{3}\right] \\
d_{b}-\text { Full depth of bruise [mm] } \\
d_{t}-\text { Depth from fruit surface to top of bruise }[\mathrm{mm}] \\
w_{1}, w_{2}-\text { Bruise widths across major \& minor axis }[\mathrm{mm}]
\end{array}
\end{aligned}
$$
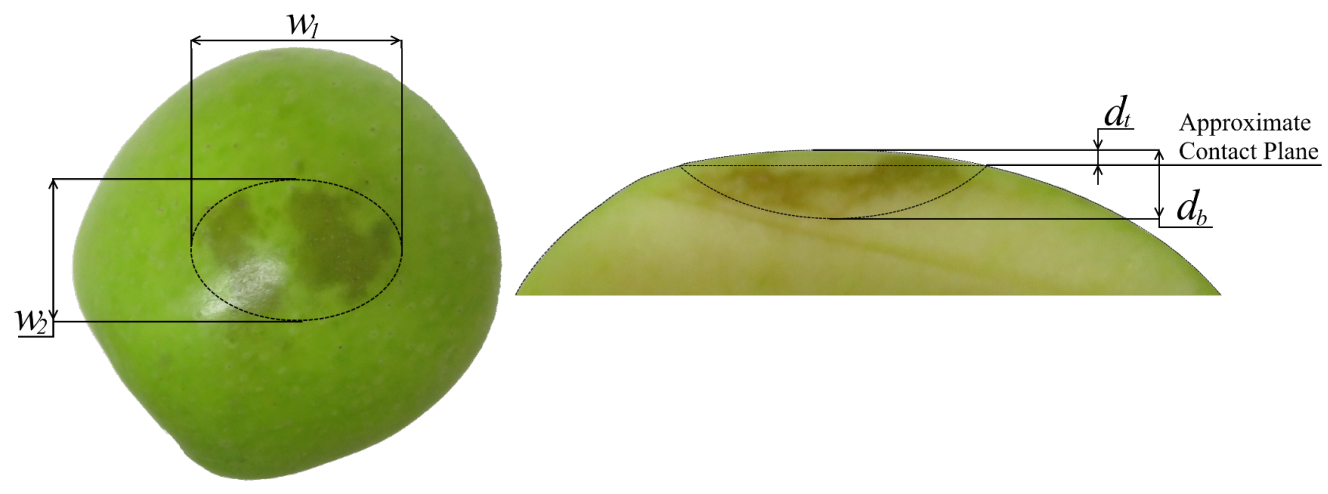

Figure 4.1: Bruise volume measurement 


\section{BRUISE PREDICTION MODEL}

\subsection{Experimental Set-Up and Procedure}

Pendulum experiments were conducted in which a relationship between peak impact force and BV could be found. The experimental set-up consisted of the same pendulum arrangement used when attaining the contact model parameters and is shown in Figure 3.3. The impact force ranged from $20 \mathrm{~N}$ to $160 \mathrm{~N}$ with the initial impact velocities ranging from 0.3 to $1.0 \mathrm{~m} / \mathrm{s}$. Apples were stored at the optimal conditions $\left(3^{\circ} \mathrm{C}, 85 \% \mathrm{RH}\right)$ as stated by Van Zeebroeck et al. (2007:11) for 24 hours before tests were conducted. Each case made use of 30 specimens. Bruises take up to 12 hours to become observable (Opara \& Pathare, 2014:10) which mean they may not be visible until they reach the consumer. Consequently, in this investigation impacted apples were allowed 24 hours (at room temperature) for their bruises to fully develop as carried out by Pang et al. (1992:229). Consistency demanded that the apples were sliced through the centre of the bruise region perpendicular to the bruise surface (Schoorl \& Holt, 1980:392).

Bruise area was quantified by assuming the area to be that of an ellipse and by making use of the major and minor widths as shown in Figure 4.1. These measurements were taken using a Vernier calliper. Bruise depth was determined by making use of the full depth of the bruise minus the depth from the fruit surface to the top of the bruise. Thus, three measures of bruising damage were recorded. Pendulum impact tests were conducted with the use of the same three impactors described in Chapter 3.2.2.

Quantification of the bruise damage due to apple-apple impacts proved to be more problematic. The same method used in Chapter 3 for studying apple-apple impacts was employed. When studying the bruises formed between two apples in contact it was observed that the impacting apple, the apple placed on the anvil or a combination of the two would bruise. This behaviour seemed to occur randomly amid apples tested on themselves or various other apple size combinations. No correlation indicating which apple or to what extent the bruise was shared between the two halves was observed. The same phenomenon was noted by Pang et al. (1992:233) who found that bruising between apple-apple contacts was, "distributed at random between the two apples, so that generally bruising was more severe on one of the two apples involved in the impact". Although the experimental set-up 
used by Pang et al. (1992:231) differed from the one used in this study and a different apple cultivar was used the same observations were made. In both studies, it was very rare for the same amount of damage to occur on both apples. However, the total amount of bruising recorded between the two apple halves in contact was found to have a good correlation with the peak force. Pang et al. (1992:233) also reported strong linear correlations between the total bruising and the absorbed energy during impact. The total amount of bruise energy required to achieve a certain level of bruising should be the same regardless of the impactor profile. The relations between impact/absorbed energy and the resulting bruise damage was thus further investigated and is detailed in Appendix $C$.

\subsection{Results}

The relationship between peak contact force and the BV is depicted in Figure 4.2 for the spherical and flat plate impactors respectively. The relations obtained for the other measures of bruise damage namely, bruise area and bruise depth are given in Appendix B (see Figure B.1 and Figure B.2) for the spherical and flat plate impactors. For the spherical impactor a strong second order trend was observed whilst for the flat plate impactor a strong linear trend was obtained. This is due to the difference in the geometrical profile of the impactor. Additionally, the onset of bruising was earlier for the spherical impactor. This was due to the larger pressure that occurs when contact commences between the spherical impactor and the specimen, as there was less surface area over which the contact was divided.

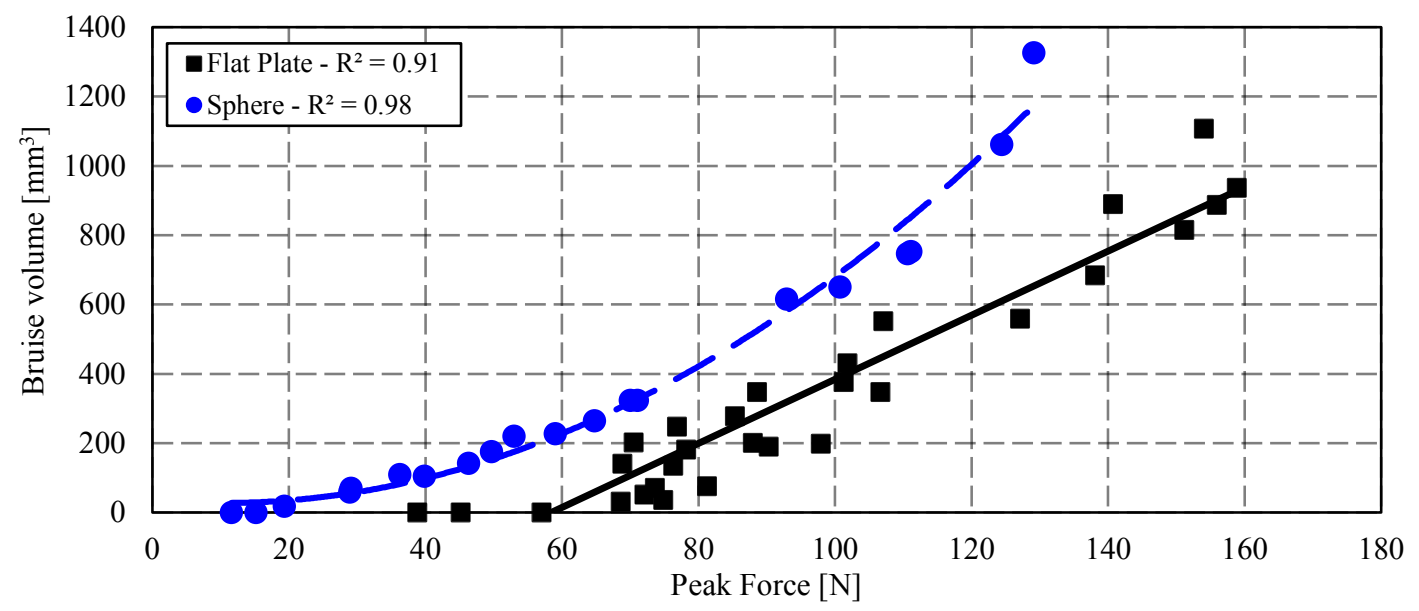

Figure 4.2: Correlation between peak force and BV for spherical and flat plate impactors 
Bruise damage correlations for the apple-apple impactor are depicted is Figure 4.3 for the BV, (see Figure B.3 for the BA and Figure B.4 for BD) with a strong linear correlation observed. In the DEM bruise prediction model, the total BV, BA and BD predicted at a contact between two apples was always split equally between the two apples. Although this meant that individual apples might show slightly different bruise levels, compared to the measurements, the total BV, BA and BD of all the apples combined should be comparable.

Good correlation coefficients were achieved as shown in Figure 4.2 and Figure 4.3, along with Figure B.1 through Figure B.4, with the BV producing the strongest correlation with peak contact force. This is in agreement with Pang et al. (1992:233) and Van Zeebroeck (2005:179).

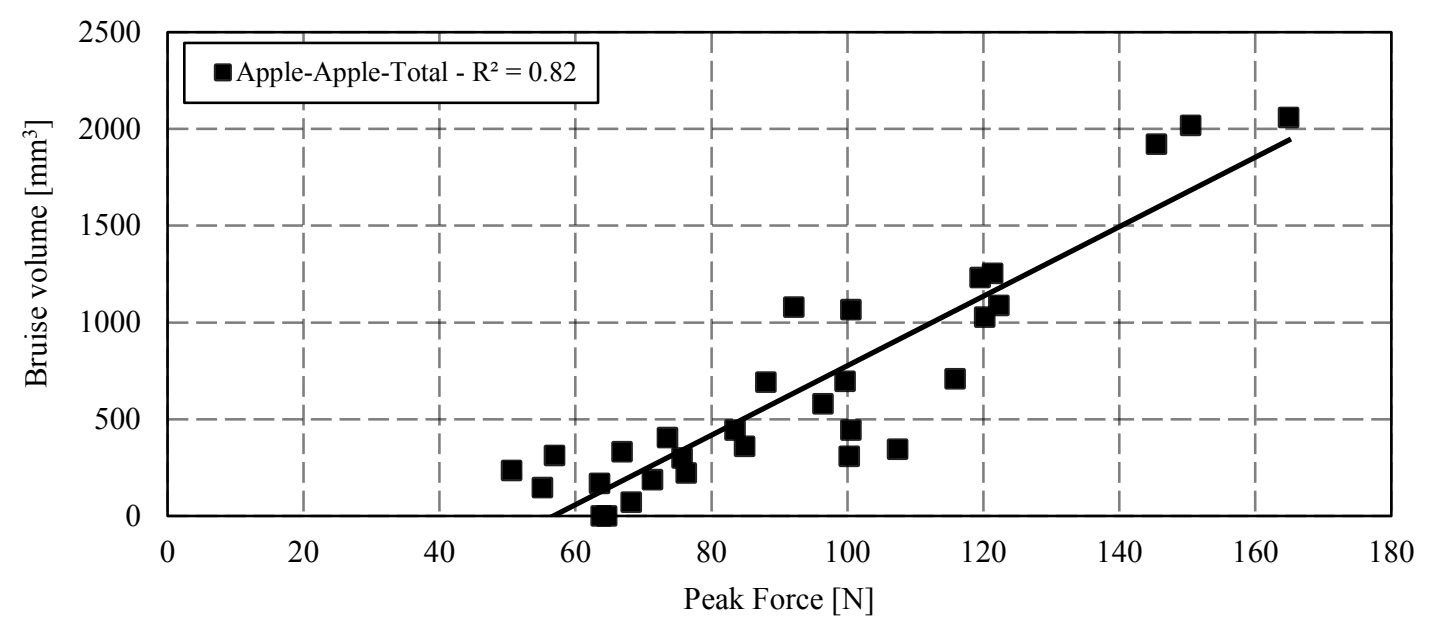

Figure 4.3: Correlation between peak force and BV for apple-apple contact

It is important to note that the use of the spherical impactor is of an academic interest. It does not represent a physically realistic contact situation that will occur to fresh produce. However, it provides an accurate means to study bruising and the influence of the radius of curvature on the contact parameters and potential bruising (as the resulting damaged area is always circular). Additionally, when a significant difference in the wall hardness or deformability is expected the applewall bruise damage correlations should be found specifically for the combination of impacting materials. This is however not required for the apple-apple correlations unless apples of different ripeness, cultivars, temperatures, et cetera are expected to be in frequent contact. 
BRUISE PREDICTION MODEL

It should be noted that for the purposes of this investigation the effect of harvest date, apple temperature, apple mass and apple radius of curvature were not considered in the development of a bruise model. Although these effects provide detailed information about the potential mechanisms present when bruises occur they would provide an unnecessary degree of complexity when incorporated into a DEM model; especially when such strong correlations between peak force and bruise damage are observed. A summary of their influence can be found in Van Zeebroeck et al. (2007:10-19).

\subsection{Onset of Apple Bruising}

Apple tissue is capable of bearing a certain amount of pressure before any permanent damage is experienced by the underlying cells. A detailed study on this behaviour was conducted by Mohsenin (1986:426-437) who defined a bioyield point below which no browning of the apple tissues can be detected. This point was defined as the first observable break in a force-deformation curve when loading apple flesh. This break in the curve is indicative of fractures within the cell structure. Furthermore, a bioyield pressure is described where the force, at the bioyield point, is taken per unit surface area. An example of the breaks (bioyield point) present in the force-deformation curve is illustrated in Figure 3.2.

This behaviour needed to be replicated in the DEM apple damage model. Consequently, a yield force was needed below which no damage would be assigned to the apple particles in the model. Mohsenin (1986:434) reported a value of $\approx 25 \mathrm{~N}$ for the yield force of Golden Delicious apples. From Figure 4.2 a similar yield force was observed for the spherical impactor. For the larger surface areas of the flat plate and the apple impactors a yield force of 50 - $60 \mathrm{~N}$ was established. This is in agreement with the yield force observed in Figure 3.2 from the quasistatic tests which also made use of a flat plate plunger.

Mohsenin (1986:426) made use of a $0.635 \mathrm{~cm}(\varnothing)$ plunger. The agreement of the spherical impactor with the values reported by Mohsenin illustrates the effect of the contacting surface area on the yield force and the use of the more universal yield pressure to describe the onset of apple bruising. However, the determination of the pressure at which the apple tissues will begin to yield is difficult as the surface area 
is always increasing as the deformation increases for realistic impacts resembling apple-wall and apple-apple impacts. Additionally, the use of yield pressures in the DEM model is also impractical and the use of a yield force specific to each contact condition is proficient. Consequently, the yield force beyond which the apples would damage was set between $50-60 \mathrm{~N}$, depending on the contact type.

\subsection{Effect of Multiple Contacts and Time between Impacts}

The developed bruise prediction model was applied to realistic apple bruising situations where the same apple may be impacted multiple times either on the same position, a new position or some overlap of old and new positions. No detailed information on the potential effect of multiple impacts on the same position on a specimen could be found in literature. Additionally, it was unknown how much damage would be present when a portion of the newly damaged area overlapped with a previously damaged area. Furthermore, the effect of the time between two impacts, with some overlapping portions, on the total damage was unknown.

Experiments were conducted by making use of the pendulum impactor and the spherical impactor described in the aforementioned sections. For each test case, nine apple samples were prepared as before. The resulting bruise damage was measured using the method described in Section 4.1. Firstly, the effect of time between two successive impacts on the same position was investigated. Nine specimens were struck up to 24 hours apart with a peak force of approximately $60 \mathrm{~N}$. The resulting BV is illustrated in Figure 4.4 which reveals that there was no discernible effect on the resulting bruise damage from impacts of the same magnitude spaced up to 24 hours apart. The resulting BA and BD are displayed in Figure B.5 and Figure B. 6 and followed the same trend.

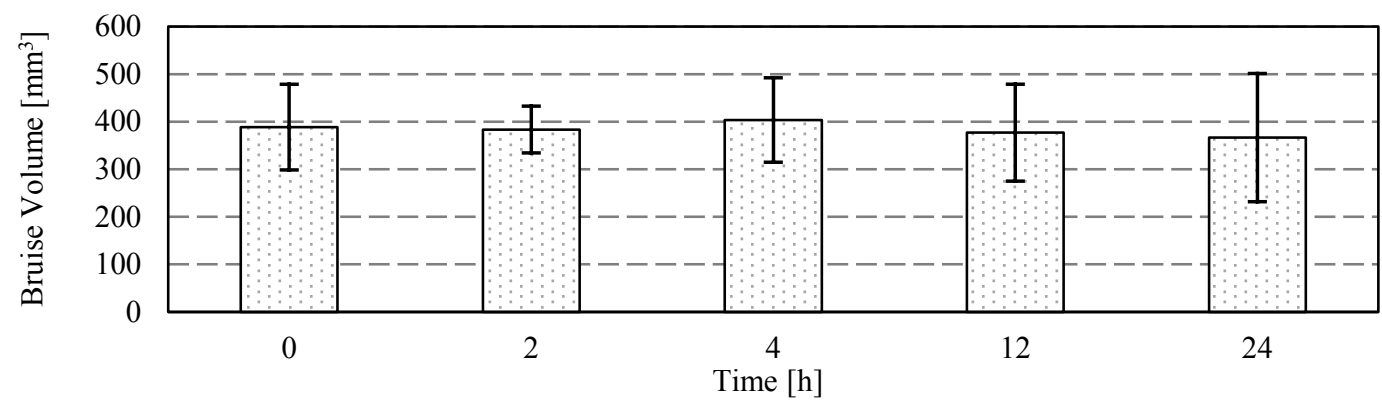

Figure 4.4: BV from two impacts spaced $0-24 \mathrm{~h}$ apart. Error bars indicate $1 \mathrm{SD}$ 
Secondly, the effect of successive immediate impacts of different magnitudes on the same position was investigated. The experiment explored the accumulative effect of multiple impacts either with an increasing force magnitude or with a decreasing force magnitude. Specimens were struck four times with the peak force increasing from $\approx 20 \mathrm{~N}$ to $\approx 100 \mathrm{~N}$ and then also decreasing with the same increments. Additionally, specimens were struck with impacts of approximately the same magnitude $(60 \mathrm{~N})$ four times. The resulting bruise volumes are shown in Figure 4.5 with the BA in Figure B.7 and the BD in Figure B.8. For comparative purposes, the results from a single impact on 16 specimens from the same batch are also shown.

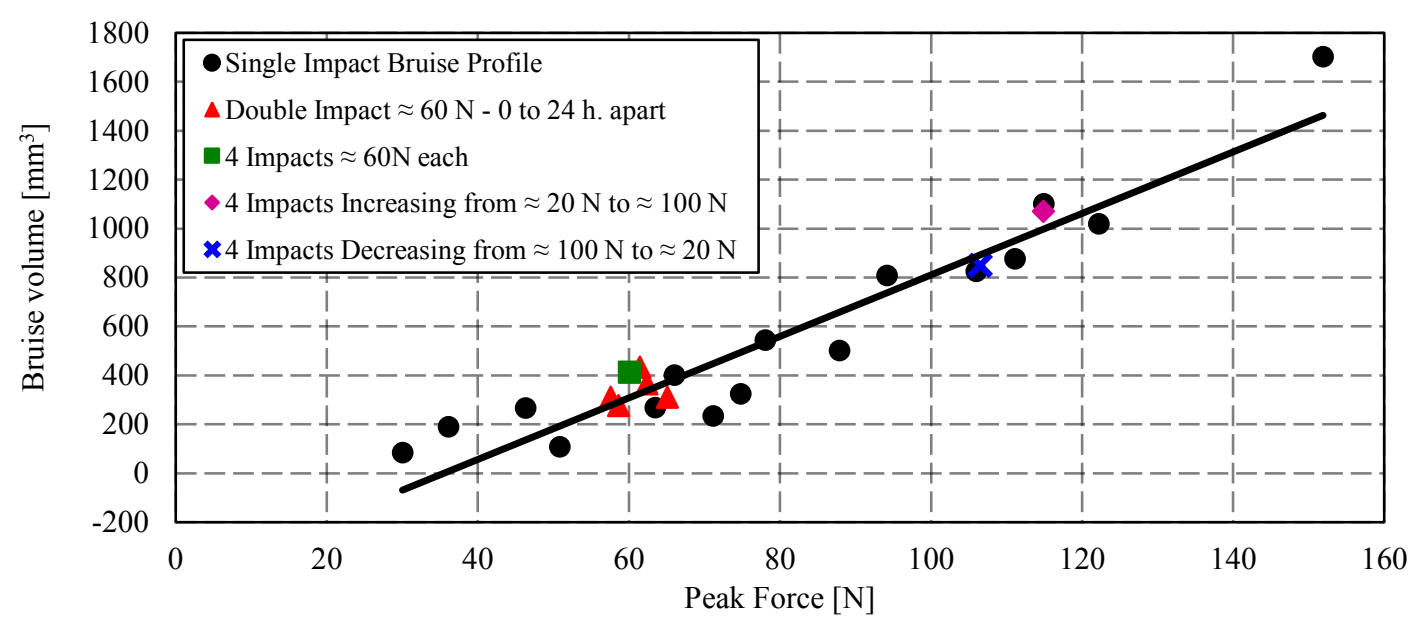

Figure 4.5: Bruise volume resulting from multiple impacts compared to single impacts

No significant difference in the bruise damage resulting from a single impact of a certain magnitude and the maximum impact amongst the range of impacts could be found. Thus, the final bruise damage corresponded to the damage associated with the largest impact within a range of multiple impacts, independent on whether the largest impact was first or last in the series. The effect of time between multiple impacts of different magnitudes was also explored with no significant effect observed (similar to the results in Figure 4.5, Figure B.7 and Figure B.8).

Consequently, the DEM bruise model could make use of the damage correlations obtained from a range of single impacts specific to the two surfaces in contact with bruise damage from multiple impacts not being accumulative. The resulting bruise 
damage would correspond to the maximum impact force on a definite location for a range of impacts. Time effects were also shown not to have an influence on the resulting damage. Finally, impacts of various sizes could occur which were not on the same precise position but overlapped to some degree. A formulation capable of accounting for such effects was explored and implemented on a post-process level. A detailed explanation of this formulation is given in Chapter 5 .

\subsection{Resulting Bruise Damage Models}

Verification of the relationships derived here was conducted in Chapter 6. The drop tests used to verify the correlations showed the need for an upper limit to be placed on the amount of bruise damage that could occur. This is more realistic to the bruise damage behaviour that would occur in practice. It is sensible to expect that a point will be reached, during loading of the apple tissues, where the force will increase but the measurable bruise damage will not significantly increase. This occurrence would not be captured unless an upper limit was placed on the amount of damage derived from the bruise damage correlations. Consequently, this upper limit was placed at $160 \mathrm{~N}$, the maximum force tested for the bruise model correlations, along with its associated bruise damage.

The resulting equations relating bruise volume, bruise area and bruise depth for the aluminium spherical impactor are given in Table 4.1. The bruise damage equations for the impacts with the flat steel plate are presented in equations 4.2 through 4.4. The resulting bruise damage correlations for apple-apple contacts are presented in equations 4.5 through 4.7 .

Table 4.1: Bruise damage correlations for the spherical impactor

\begin{tabular}{l|lc}
\multicolumn{1}{c}{$\begin{array}{c}\text { Bruise Damage } \\
\text { Measure }\end{array}$} & \multicolumn{1}{c}{ Regression Equation } & $\begin{array}{c}\text { Correlation } \\
\text { Coefficient } \\
{\left[\mathbf{R}^{2}\right]}\end{array}$ \\
\hline Bruise Volume $\left[\mathrm{mm}^{3}\right]$ & $B V=0.0813 P F^{2}-1.685 P F+36.155$ & 0.9807 \\
Bruise Area $\left[\mathrm{mm}^{2}\right]$ & $B A=-0.0156 P F^{2}+8.3406 P F-29.764$ & 0.9364 \\
Bruise Depth $[\mathrm{mm}]$ & $B D=-0.0002 P F^{2}+0.0932 P F-1.0082$ & 0.9799
\end{tabular}

Good correlation coefficients are found and given in Figures 4.2 and 4.3 along with Figures B.1 through B.4. The relationships found for the apple-plate impacts have superior correlations when compared to those of the apple-apple impacts. This is 
as expected when keeping in mind the higher degree of variability associated with two biological materials in contact.

The developed bruise prediction model was subsequently programmed (with the use of $\mathrm{C++}$ ) as an intrinsic within the $P F C^{3 D}$ programme. The use of custom intrinsics within $P^{3} C^{3 D}$ offer serval distinct advantages most pertinently execution speeds of 10 to 100 times faster as well as direct access to internal data structures and methods not necessarily available through the predefined interface $\left(P F C^{3 D}, 2016\right)$. The resulting bruise damage models are given in equations 4.2 through 4.7:

Apple - Wall:

$$
\begin{aligned}
& B V=\left\{\begin{array}{cl}
0 & \text { for } P F \leq 59 \mathrm{~N} \\
9.2418 P F-540.01 & \text { for } 59 \mathrm{~N}<P F<160 \mathrm{~N} \\
938.678 & \text { for } P F \geq 160 \mathrm{~N}
\end{array}\right. \\
& B A=\left\{\begin{array}{cl}
0 & \text { for } P F \leq 51 \mathrm{~N} \\
10.176 P F-487.82 & \text { for } 51 \mathrm{~N}<P F<160 \mathrm{~N} \\
1140.34 & \text { for } P F \geq 160 \mathrm{~N}
\end{array}\right. \\
& \text { for } P F \geq 160 \mathrm{~N} \\
& B D=\left\{\begin{array}{cl}
0 & \text { for } P F \leq 51 \mathrm{~N} \\
4.6662 \ln P F-17.194 & \text { for } 51 \mathrm{~N}<P F<160 \mathrm{~N} \\
1140.34 & \text { for } P F \geq 160 \mathrm{~N}
\end{array}\right.
\end{aligned}
$$

Apple-Apple:

$$
\begin{gathered}
B V=\left\{\begin{array}{cl}
0 & \text { for } P F \leq 57 \mathrm{~N} \\
\frac{1}{2}(17.955 P F-1018.9) & \text { for } 57 \mathrm{~N}<P F<160 \mathrm{~N} \\
926.95 & \text { for } P F \geq 160 \mathrm{~N}
\end{array}\right. \\
B A=\left\{\begin{array}{cl}
0 & \text { for } P F \leq 51 \mathrm{~N} \\
\frac{1}{2}(17.049 P F-712.56) & \text { for } 51 \mathrm{~N}<P F<160 \mathrm{~N} \\
1007.64 & \text { for } P F \geq 160 \mathrm{~N}
\end{array}\right. \\
B D=\left\{\begin{array}{cl}
0 & \text { for } P F \leq 51 \mathrm{~N} \\
\frac{1}{2}(0.0792 P F-1.6944) & \text { for } P F \geq 160 \mathrm{~N} \\
5.4888 & \\
B V-\text { Bruise Volume }\left[\mathrm{mm}^{3}\right] \\
B A-\text { Bruise Area }\left[\mathrm{mm}^{2}\right]
\end{array}\right. \\
B D-\text { Bruise Depth }\left[\mathrm{mm}^{2}\right]
\end{gathered}
$$


NUMERICAL IMPLEMENTATIONS

\section{NUMERICAL IMPLEMENTATIONS}

The DEM model predicting apple damage was next constructed within the numerical environment. The appropriate mechanical parameters were described in Chapter 3. The coupling between the forces obtained through DEM and the bruise damage is presented in Chapter 4 . The resulting bruise prediction tool was subsequently implemented in the commercial DEM programme $P F C^{3 D}$ from Itasca Consulting Group, Inc. $\left(P F C^{3 D}, 2016\right)$. In this chapter, apple shape and size representation, implementation of the contact model and the management of overlapping bruises are presented.

\subsection{User Defined $P F C^{3 D}$ Model Components}

$P F C^{3 D}$ gives the user control over the model through an embedded programming language called $\mathrm{FISH}$. Through $\mathrm{FISH}$ the user has access to manipulate almost all data and processes within the time cycle operations. Pre-defined call-backs are available to execute a user-defined $F / S H$ function in response to a specific event, for example when a contact is created or deleted, directly before or after the contact forces are updated, or at the end of the current time step $\left(P F C^{3 D}, 2016\right)$. $P F C^{3 D}$ additionally allows users to create their own contact models permitting researchers to simulate contact interactions as they require. To this end, $P F C^{3 D}$ provides a contact model framework that is common to all contact model definitions which the user's model must follow in order to function correctly within the existing programme structure. These user-defined contact models are programmed in $\mathrm{C}++$ and loaded as a dynamic-link library (DLL) plug-in.

\subsection{Representation of Particle Shape and Size}

Accurate representation of a particle's geometrical profile in DEM plays an important role in correctly capturing the dynamic behaviour of real particles. The correct representation of a particle's shape is also of vital importance for accurate contact detection and the precise computation of contact forces (Favier et al., 1999:467). Consequently, shape representation will greatly influence the ability of DEM to predict the impact damage and fruit bruising which are derived from contact force interactions. 
Practically, apples have an infinite amount of geometrical profiles if individuals are studied on a sufficiently detailed level. However, when observed on a larger macroscopic scale a clear well-defined unique shape can always be identified with different cultivars often exhibiting particular profiles. This notion is true for almost all of the natural particles typically studied and modelled using DEM with manufactured particles (i.e. pharmaceuticals, plastic-pellets, et cetera) being the exception. Additionally, a few distinguished recurring shapes can usually be identified for the particles under examination.

Consequently, ten apple profiles were selected at random and captured using a 3D laser scanner (NextEngine, Model 2020i - dimensional accuracy \pm 300 micron). As the reoccurring apple cultivar investigated in literature is the 'Golden-Delicious', ten distinct 'Golden-Delicious' shapes were recorded. Six of the mesh shapes characteristic of the profiles generated are shown in Figure 5.1. The profiles were exported in stereolithography (STL) format with the number of triangular facets per apple ranging between 22660 and 26398 resulting in all the necessary details of the profiles being captured.
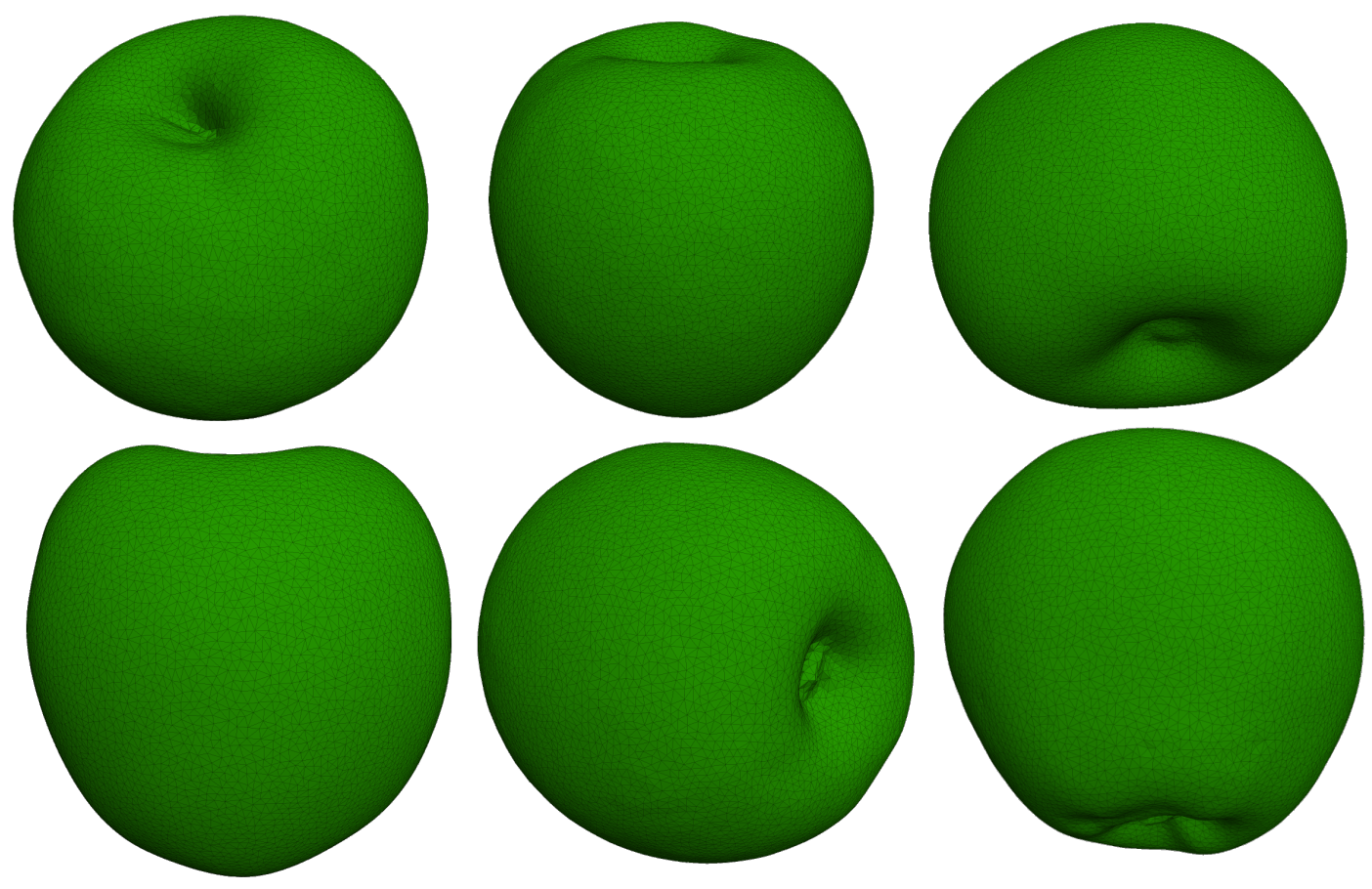

Figure 5.1: Mesh profile of six apples obtained through laser scanning 
The recorded profiles could be used throughout the investigation to generate the particle shapes required in the DEM environment. The exact volumetric size of the apple profiles was not as imperative since the $P F C^{3 D}$ software package is capable of scaling the profile to a volume equivalent diameter of a sphere. Additionally, a size range could be specified in which different profiles could be scaled to different equivalent diameter sizes. The natural variation of apple sizes that typically occurs within a given batch could therefore easily be replicated. Hereafter, the "Bubble Pack" procedure, described in Section 2.5.2, was followed to generate the "clump templates" of the apples. A clump template is based on an imported STL file of the particle profile and was generated only once for each of the ten apple profiles. The templates were then easily imported, scaled and used, in equal proportion, to generate the number of apples required for a simulation.

The desired precision of the generated clump relative to the given surface description can additionally be specified in the Bubble Pack algorithm. This is defined as an angular measure of smoothness where $0^{\circ}$ corresponds to the roughest measure of smoothness and $180^{\circ}$ smoothest or least rough (Taghavi, 2011:6). The closer the angle is defined to the upper limit of $180^{\circ}$ the smoother the pebble distribution and consequently the greater the number of pebbles within a clump.

Unfortunately as the number of pebbles per clump increased (and accordingly the smoothness of the clump surface) so too did the computational effort required to perform a given simulation. The influence of the number of pebbles per clump on the computational time required for a computational cycle was thus investigated.

A simple simulation was created where an apple clump would be dropped from a fixed height and allowed to settle until a static equilibrium state was achieved. The contact model was set to the default linear model and the computational time step size was fixed. Various measures of increasing smoothness were specified and the resulting number of seconds required per cycle (a single time step increment) determined. The increase in the number of seconds necessary to complete the calculation of one cycle is depicted in Figure 5.2 as a function of the smoothness parameter. 


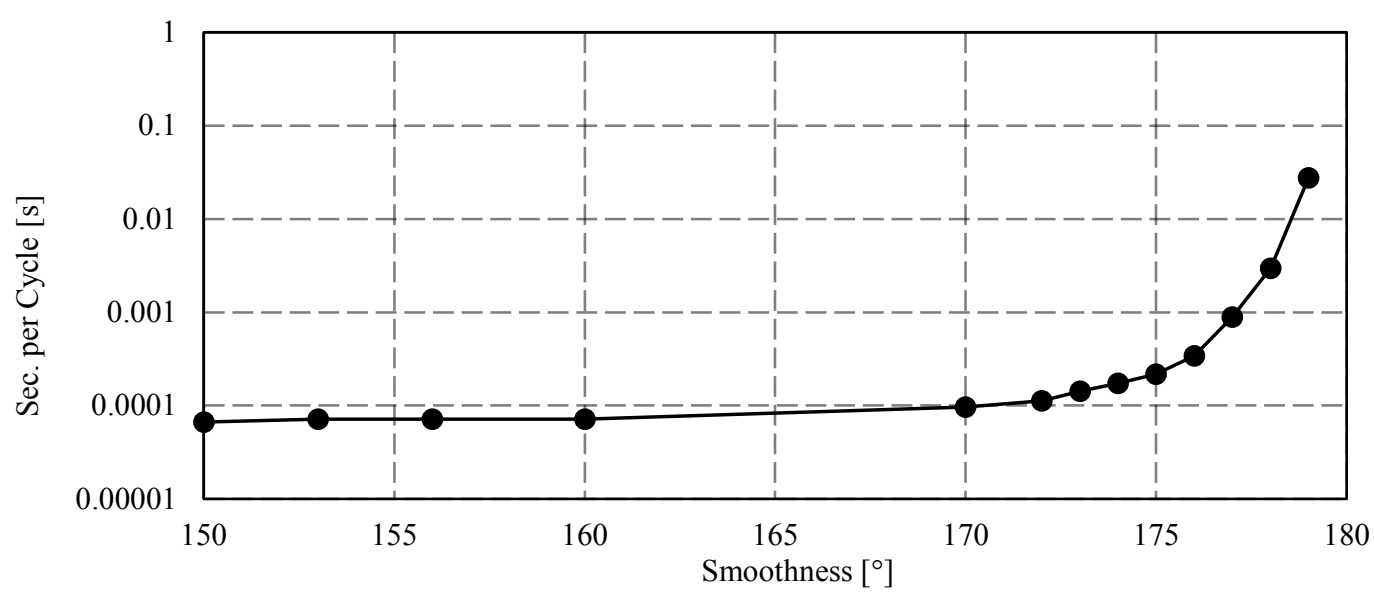

Figure 5.2: Computational time required with varying clump angle of smoothness

The results illustrate the exponential increase in computation time as the smoothness factor reached its upper limit. Limiting this increase in calculation time would greatly expedite all ensuing simulations that needed to be performed. This was further compounded when the computational time exponentially increased as the number of clumps in a given simulation increased. This is illustrated in Figure 5.3.

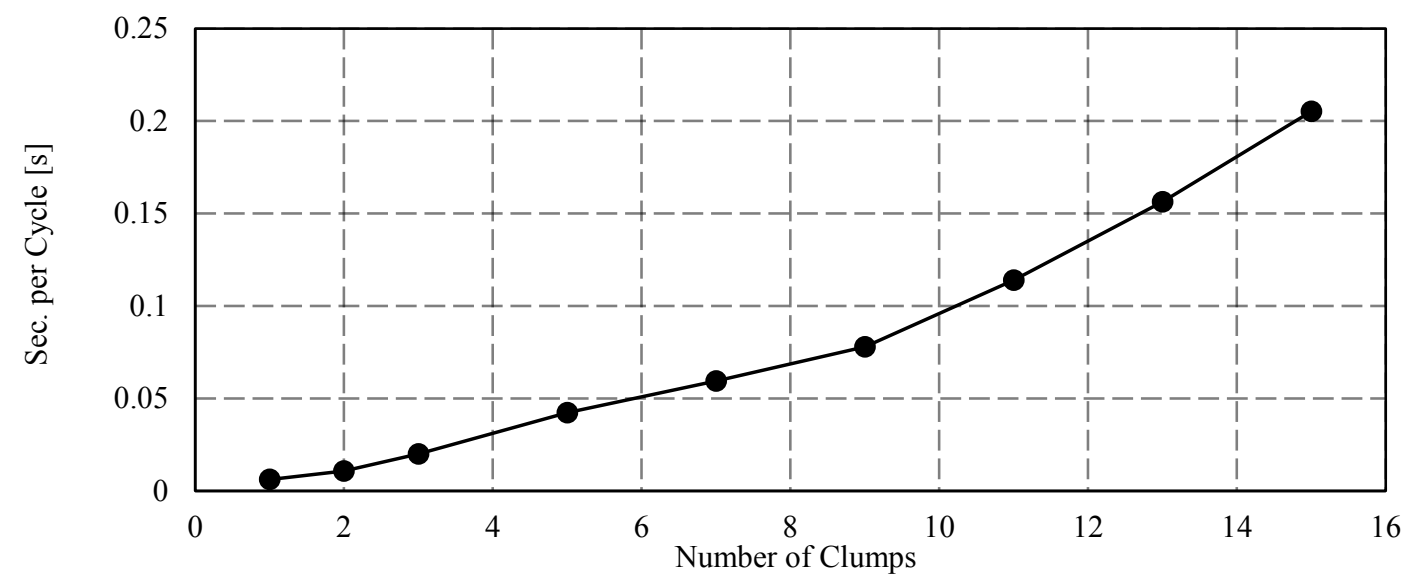

Figure 5.3: Computational time required with an increasing number of clumps in a simulation

As a consequence, an angular measure of smoothness of $170^{\circ}$ was specified for the generation of the ten apple clump templates. This smoothness angle offered the most accurate shape representation of the true apple surface before the sudden sharp increase in computational requirements, shown in Figure 5.2. One of the resulting apple clump templates is illustrated in Figure 5.4. 


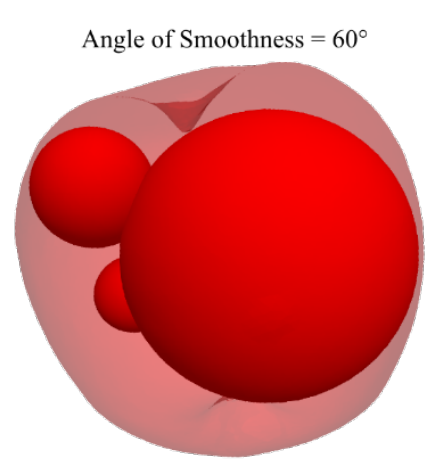

Angle of Smoothness $=170^{\circ}$

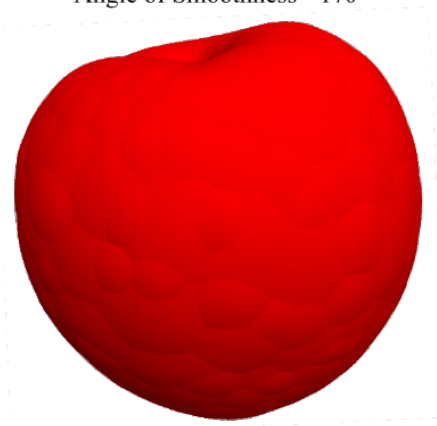

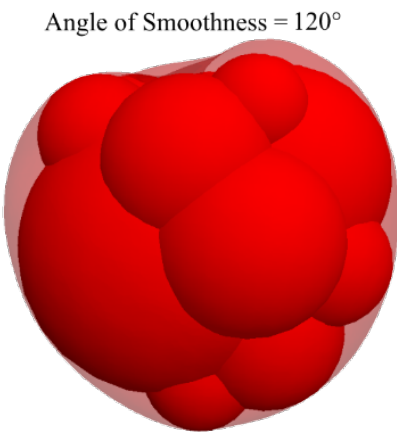

Angle of Smoothness $=179^{\circ}$

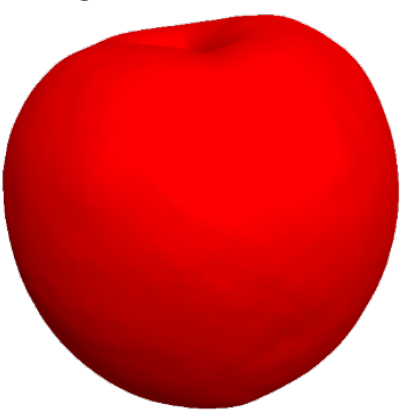

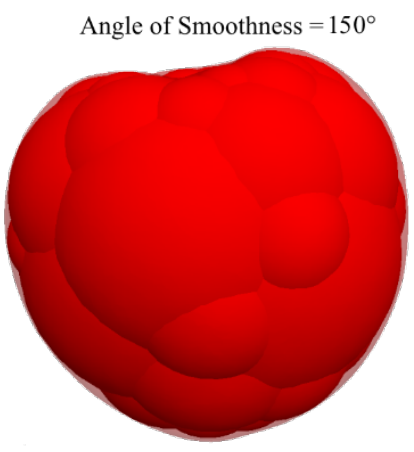

Equivalent Scanned Mesh File

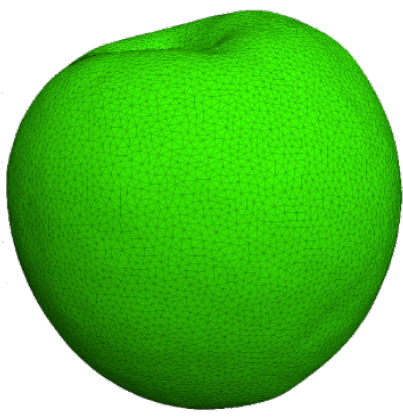

Figure 5.4: Comparison of clumps with differing angles of smoothness $\left[^{\circ}\right]$

Here, a comparison between the chosen angle of smoothness with the maximum angle of $179^{\circ}$ and the scanned mesh profile is also illustrated. The clump with this maximum allowable angle of smoothness almost exactly matched the scanned mesh file but contained 14668 overlapping spheres (pebbles) whereas the clump with an angle of smoothness of $170^{\circ}$ still achieved an agreeably accurate shape representation whilst making use of only 375 pebbles.

As a result, the clump of $179^{\circ}$ made use of almost eight times more overlapping spheres to represent a single apple clump which greatly increased computational effort. An angle of smoothness of $170^{\circ}$ offered the best accommodation of both accurate shape representation and computational time.

\subsection{Contact Model Implementation}

The Kuwabara and Kono normal viscoelastic contact force model, presented by equation 3.3, was subsequently programmed in $C++$ and loaded as a plug-in $D L L$ file into the $P F C^{3 D}$ programme. The use of $P F C^{3 D}$ 's implementation of the 
Mindlin-Deresiewicz tangential contact force model, equation 2.4, was incorporated into the resulting model as discussed in Section 2.6.

The contact model was written in a generic way. In other words, the appropriate normal elastic stiffness was automatically calculated by the contact model algorithm and incorporated the radius of curvature of the impacting objects. Thus, only the elastic (Young's) modulus was specified for the computation of the normal elastic constant. The radius of curvature of the impacting body was determined from the volume equivalent radius of a sphere for the clump representing the whole apple. Note that this is different from the default implementation in $P F C^{3 D}$ where the radius of the contacting pebble would be used as the radius of curvature. The radii of curvature of the plane walls was taken as infinite $\left(R_{\text {wall }} \rightarrow \infty\right)$. The resulting input parameters for the contact model representing the mechanical behaviour of apples are presented in Table 5.1. The derivation of the material constants is given in Chapter 3.3 and the description of the dynamic friction coefficients is given in Chapter 2.3.

Table 5.1: Resulting contact model properties as specified in $P F C^{3 D}$

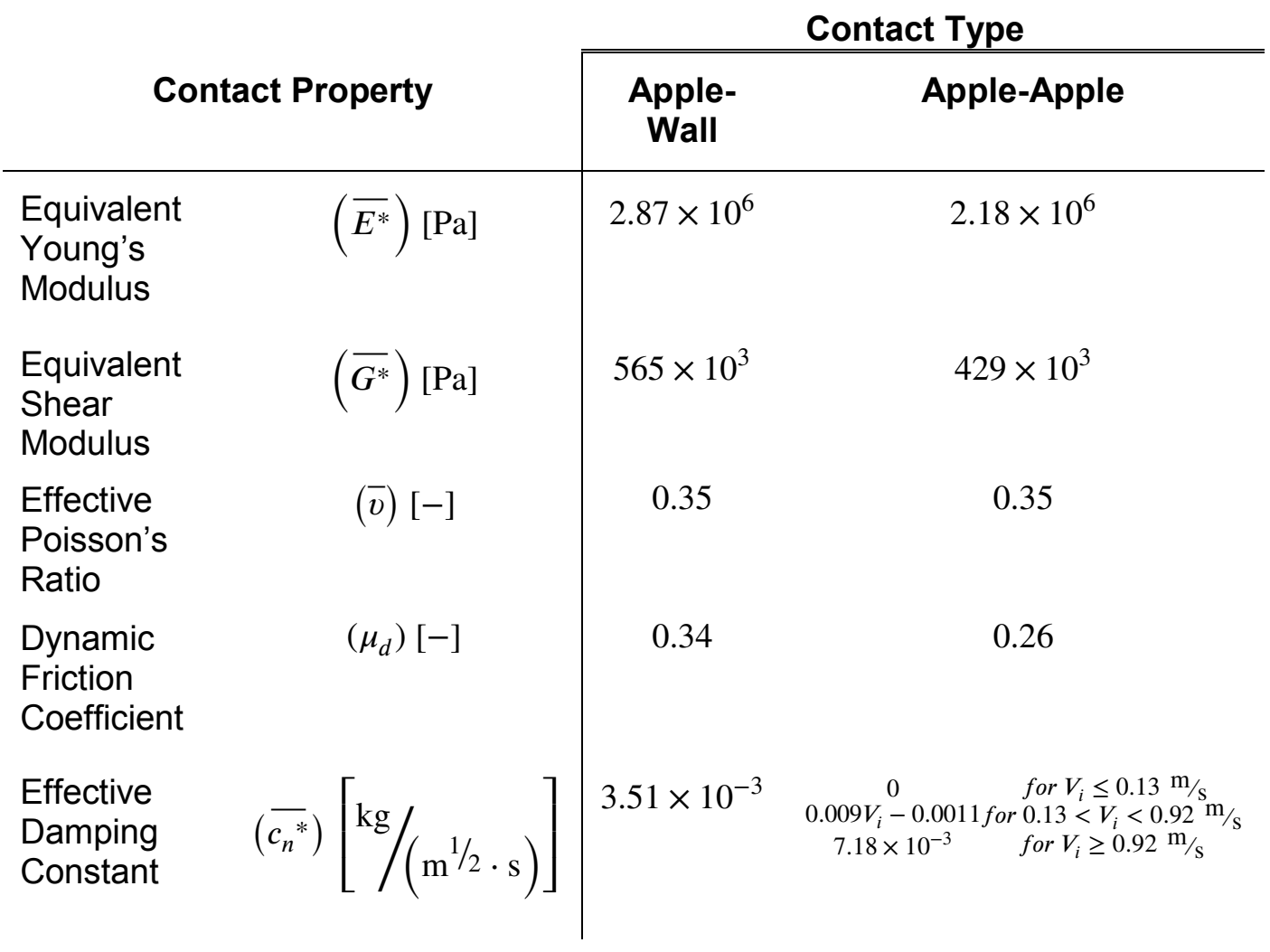


NUMERICAL IMPLEMENTATIONS

\subsection{Single Point Contact Assignment}

Within $P F C^{3 D}$ particles are donated as bodies with three types of bodies occurring: balls, clumps and walls. Bodies are made up of one or more pieces. Balls comprise a single piece called a sphere. Clumps can consist of many spheres called pebbles, which are allowed to overlap to model arbitrary shaped bodies. Walls are composed of multiple triangles called facets. New contacts are automatically formed when overlap is detected between the following pieces: ball-ball, ballpebble, pebble-pebble, ball-facet or pebble-facet. Clumps with a very accurate shape representation (containing a large number of pebbles) will result in a large number of overlapping pebbles. Consequently, a large number of contact forces will be registered in close proximity to each other as overlap occurs between pieces. An example of this is shown in Figure 5.5a where a single apple makes contact with a facet (wall). This is a well-known disadvantage of using clumps. Kruggel-Emden et al. (2008) and Höhner et al. (2011) showed that the contact behaviour of a clump (general spherical shape) dropped onto a wall with multiple contacts was different from that of a single ball with the same properties and a single contact force.

In this study the contact properties and bruise correlations were obtained for appleapple and apple-facet contacts using the pendulum device (referred to as the Direct Measuring Approach, Coetzee, 2017). During the experiments, it was assumed that a single point contact occurred, even if the physical contact occurred over a finite area. In order to model this behaviour more accurately, the number of contacts on a clump had to be reduced using a so-called single point contact algorithm, specifically developed for this purpose. Although initial test runs showed that the dynamic bulk behaviour of a number of apples (in a rotating drum for example) was not expressively altered by allowing multiple contacts, it had a significant effect on the predicted bruising. The BV was related to the peak contact force and with multiple contacts allowed, it was possible that none of the contact forces was above the threshold where damage started to occur. In other words, multiple contacts would be registered, but the apple will show no signs of bruising in the simulation. On the other hand, if the multiple contacts were to be replaced by a single contact, the force magnitude would be such that the correct BV would be registered. 
In experimental and numerical terms, there can only be a single contact between two spherical particles or between a spherical particle and a flat wall. However, apples are not perfectly spherical and in physical terms, more than one contact is possible between two apples or between an apple and a wall. For example if the apple is resting on its top or bottom on a wall, as depicted in Figure 5.5. In order to model this accurately, the number of contacts between two apples or an apple and wall should be reduced, but not limited to a single contact. For this purpose a contact proximity radius was defined. When two pieces made contact for the first time, that contact was registered and contact forces allowed to be generated. All subsequent contacts that formed between these two pieces were checked against existing contacts. If the new contact was within the proximity radius of an existing contact, the new contact was given a null material model which would result in zero contact forces. However, if the new contact fell outside the proximity radius of all existing contacts, it was assigned to the default (viscoelastic) contact model. This is illustrated in Figure 5.5b where the number of contacts was reduced from 14 to 4 between the apple and the wall. The contact proximity radius was based on the mean bruise width measured in the experiments (Chapter $4, \bar{w}=13.7 \mathrm{~mm}$ ) and set to $7.5 \mathrm{~mm}(\varnothing 15 \mathrm{~mm}$ ). A sensitivity study showed this to be a meaningful assumption (Appendix $G$ ) with significant variations of the proximity radius only producing slight deviations in the damage results and no significant change in the dynamic behaviour. A comparison between the bruise damage resulting from simulations involving clumps and simulations involving balls (where a single point contact is registered by default) is given in Appendix $G$ with vastly superior results witnessed for the clumps.
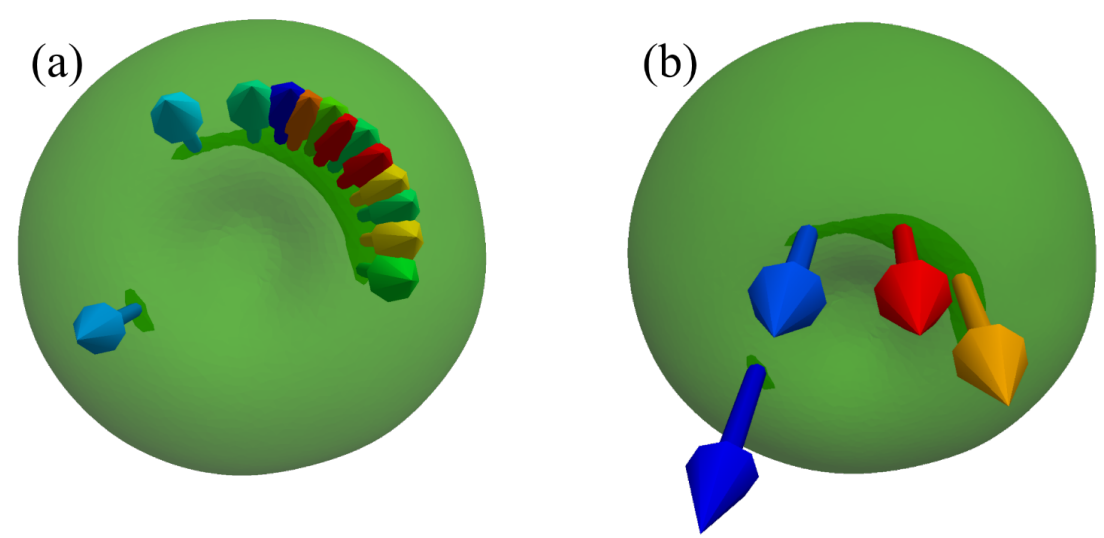

Figure 5.5: Comparison of contact forces (a) with and (b) without single point contact assignment 
When a new model was generated, two three-dimensional matrices were initialised namely, ClumpClumpTracker(ncl,ncl,nct) and ClumpWallTracker(ncl,nwl,nct) where the dimensions were the number of clumps in the model $(n c l)$, the number of walls in the model $(n w l)$, and the expected number of contacts between any two pieces in each case $(n c t)$. These matrices were used to keep track of active contacts between two contacting pieces, either clump-clump or clump-wall. For example the entry ClumpClumpTracker $(4,5,1)=21$ would indicate that the first active contact between clump 4 and clump 5 had a unique ID of 21 . ClumpClumpTracker $(4,5,2)=98$ would indicate that the second active contact between these two clumps had the ID of 98 ( $P F C^{3 D}$ automatically assigns the contact ID when a new contact forms).

For each contact, $P F C^{3 D}$ provided the pointers to the two contacting pieces. However, the user has no control over the assignment of pointers, and in this case, $P F C^{3 D}$ might provide clump 4 as piece 1 and clump 5 as piece 2, or vice versa. For this reason, the data stored in the matrix should be symmetric such that ClumpClumpTracker $(4,5, z)=$ ClumpClumpTracker $(5,4, z)=21$, for example. This meant that when a new contact formed between these two pieces, the existing contacts could be checked for proximity at either of the two entries above. Contacts between clumps and walls were stored in ClumpWallTracker and followed the same logic.

The basic outline of the single contact assignment and remove algorithms is shown in Figure 5.6 through pseudocode; additionally a flowchart illustrating the programme structure is given in Figure D.2 in Appendix D. The Single Contact Assignment function was registered as a call-back and automatically called each time a new contact with ID $i$ was formed by $P F C^{3 D}$ 's contact detection algorithm.

The contact type was checked to be either pebble-pebble (clump-clump) or pebblefacet (clump-wall). In each case the appropriate ID's (pointers) were obtained for the two contacting pieces. Next, the local coordinates of the contact were obtained. The for loop ran through all the existing active contacts between these two pieces and checked if the newly formed contact was within the proximity radius of any of the existing contacts. If this was the case, the contact would be assigned the "null" material model which would not develop contacting forces. If the newly formed 
contact was not within the proximity radius of any existing active contact, it would be assigned the viscoelastic contact model by default with the appropriate parameter values depending on the contact type. In this case, it would also be registered as an active contact between the two pieces by entering the contact ID into the first available open slot in either the ClumpClumpTracker or the ClumpWallTracker matrix as appropriate.

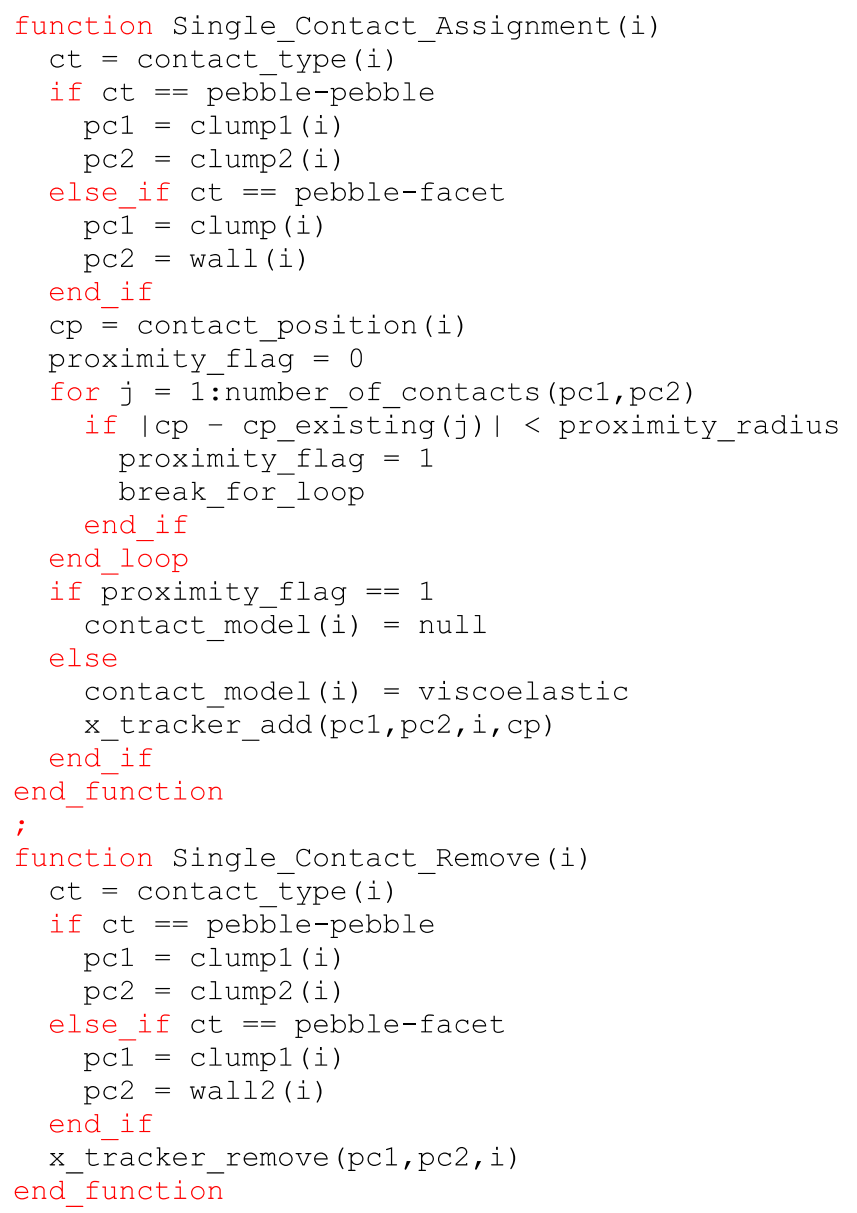

Figure 5.6: Pseudocode outlining the single contact assignment and remove algorithms

The Single Contact Remove function was registered as a call-back and automatically called each time a contact with ID $i$ was deleted by $P F C^{3 D}$. The contact type was checked and in each case the appropriate ID's were obtained for the two contacting pieces. The contact ID was then removed from the appropriate tracker matrix. 
NUMERICAL IMPLEMENTATIONS

\subsection{Force and Bruise Damage Coupling}

Once the contact model was incorporated into the $P F C^{3 D}$ programme the bruise damage formulations were coupled to the resulting peak forces. This was accomplished in two stages: first the contact data for each clump was stored during the simulation run and then written to text files as output and secondly a postprocessing algorithm was used to determine bruise overlap. The first step is described in this section.

FISH allows the user to add an extra variable to the existing clump class (and other classes). Using this feature, a matrix called the damage matrix, was assigned to each clump in the model. This matrix had a number of columns to store the unique ID, type, global position, local position, maximum force, BV, BA and BD for each contact in a row.

At the start of the simulation, a damage matrix was initialised for each clump as described above. Using a call-back, the function Contact_Data was automatically executed during each time step, immediately after the contact forces were updated. The basic algorithm of this function is shown in Figure 5.7 through pseudocode, additionally a flowchart illustrating the programme structure is given in Figure D.1 in Appendix D. The outer for loop ran over all the clumps in the model, each with a unique identification number $i$. The inner for loop ran over all the contacts $j$ surrounding clump $i$ at this point in time. Using the contact ID, the contact type (either pebble-pebble or pebble-facet) and normal force could be obtained. Note that in the algorithm presented here, contact and clump numbers were used for illustration purposes, in the code - pointers were used to access and update specific contact and clump parameter values. Based on the contact type and normal force, the BV was then calculated using the experimentally obtained correlation. Depending on the correlation, if the normal force was below a certain value, no bruising would occur and if this was the case, the algorithm skipped to the next contact. However, if a BV larger than zero was registered, the BA and BD were calculated based on the normal force and the contact type. The ID of the contact under investigation was then searched in the damage matrix. If it was found, it meant that this was an existing contact for this clump and the maximum force, $\mathrm{BV}, \mathrm{BA}$ and $\mathrm{BD}$ stored in the matrix were updated if it increased in 
magnitude. If the contact was not found in the matrix, it was a new contact and it's ID, global position (model coordinates), local position (clump principal axes), normal force, $\mathrm{BV}, \mathrm{BA}$ and $\mathrm{BD}$ were stored as a new entry in the damage matrix.

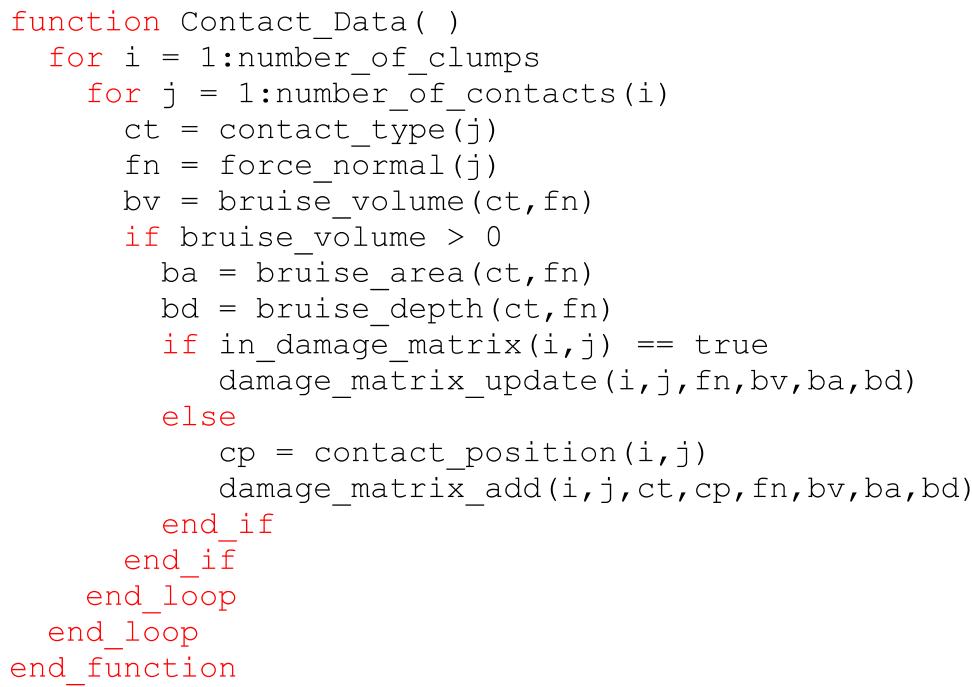

Figure 5.7: Pseudocode outlining the contact data algorithm

This function was automatically executed during each time step and at the end of the simulation run, the damage matrix of each clump was written to a text file for post-processing.

\subsection{Overlap Formulation}

When apples are involved in realistic contact situations it is inevitable that they will be impacted on positions that are in close proximity to one another. This will lead to a degree of overlapping of the resulting bruise damage. In order to quantify the resulting bruise damage, a formulation was developed that could account for the total damage that was present when bruises overlapped. From Chapter 4.5 it was observed that the resulting bruise damage corresponded to the maximum impact amongst a range of impact forces.

Accordingly, a numerical method was developed for comparing bruises with a degree of overlap, where the largest bruise was completely preserved. The basic algorithm of this function is shown in Figure 5.8 through pseudocode, additionally a flowchart illustrating the programme structure is given in Figure D.3 in 
Appendix D. The overlap area between two bruises in question was calculated by making use of the lens area of two overlapping circles (the BA was always assumed to be a circle). The resulting BA for the smaller bruise was subsequently reduced by subtracting the overlap area from the total original area of the bruise. Subsequently, a ratio between the total and reduced area was determined. This ratio was in turn used to calculate the reduced BV. For the BD the maximum depth was always conserved.

The overlap formulation was implemented on a post-process level within MATLAB (2015). To this end, all the bruises and their locations were recorded within $P F C^{3 D}$ as they occurred. This data was subsequently imported into MATLAB where the clumps' bruise data, associated STL files (scanned surface mesh), volumes and inertial axes were synchronised.

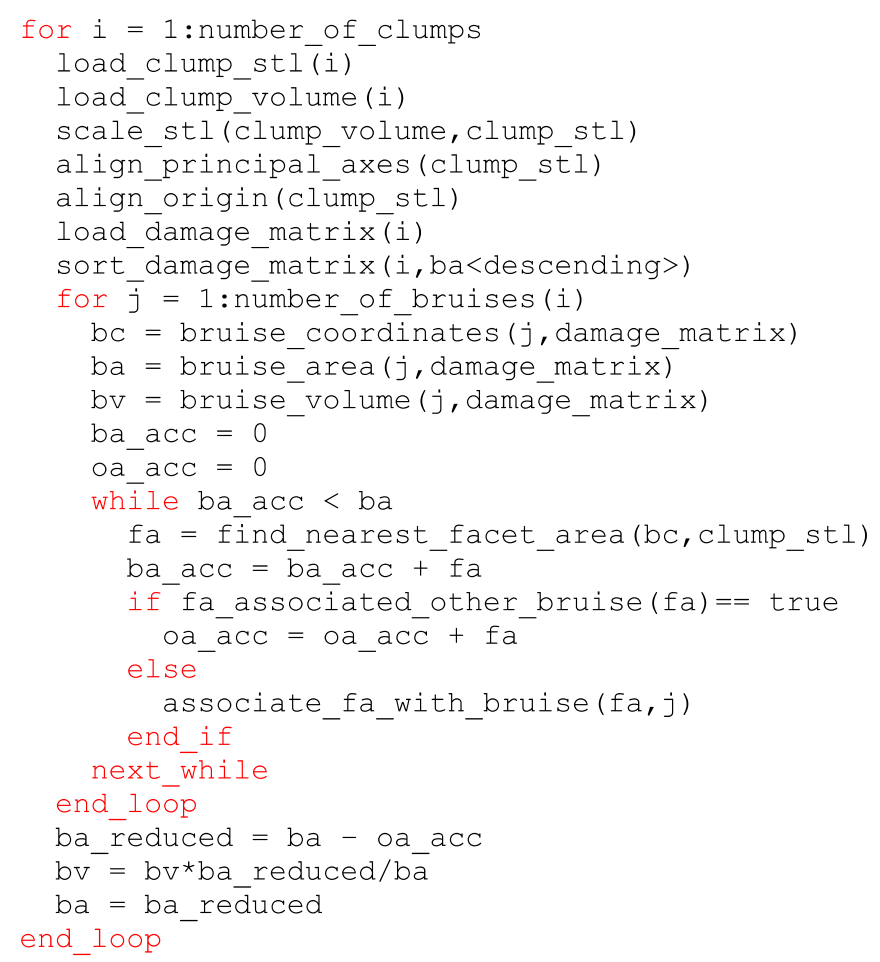

Figure 5.8: Pseudocode outlining the bruise overlap algorithm

The bruise overlap algorithm cycled through all the clumps in the model and for each loaded the appropriate STL file and the clump volume from a data file. The original STL mesh was based on the scanned apple and had to be scaled geometrically to fit the size of the specific clump. This was achieved using the 
clump volume and the volume of the original STL mesh. The next step was to rotate the STL mesh so that the principal axes of the clump (as calculated by PFC ${ }^{3 D}$ and exported in a data file) aligned with the coordinate system in MATLAB. This had to be done since the coordinates of all contacts (bruises) were recorded in the clump local principal axes. The last step in manipulating the STL mesh, was to set the centre of gravity of the clump equal to the origin of the coordinate system in MATLAB, Figure 5.9.

The bruise damage matrix was then loaded for the clump under investigation. It was shown experimentally that with overlapping bruises the characteristics of the largest bruise should be retained. Therefore, the damage matrix was sorted with the bruise areas in descending order. The inner for loop (Figure 5.8) then cycled over all the bruises registered for this clump. For each bruise, the coordinates were extracted from the damage matrix. The accumulated BA and accumulated overlap area were reset and the while loop cycled over all the STL facets until the accumulated area was equal or greater than the BA. This was achieved by finding the nearest facet to the bruise coordinates and its area. The facet was also checked if it was already assigned to another (larger) bruise. If this was the case, the overlap area was accumulated by adding the facet area to it, otherwise it was associated to the current bruise. After the while loop executed, the reduced BA could be calculated by subtracting the overlap area from the original BA. Using the ratio of the reduced $B A$ to the original area, the $B V$ was reduced. The BD was simply kept as the maximum depth for the bruise. An illustration of this facet-wise bruise implementation is given in Figure 5.9. The visualisation of apple STL meshes comprising of multiple bruises (typically found within a simulation) in the post-processing environment is given in Figure D.4 in Appendix D.
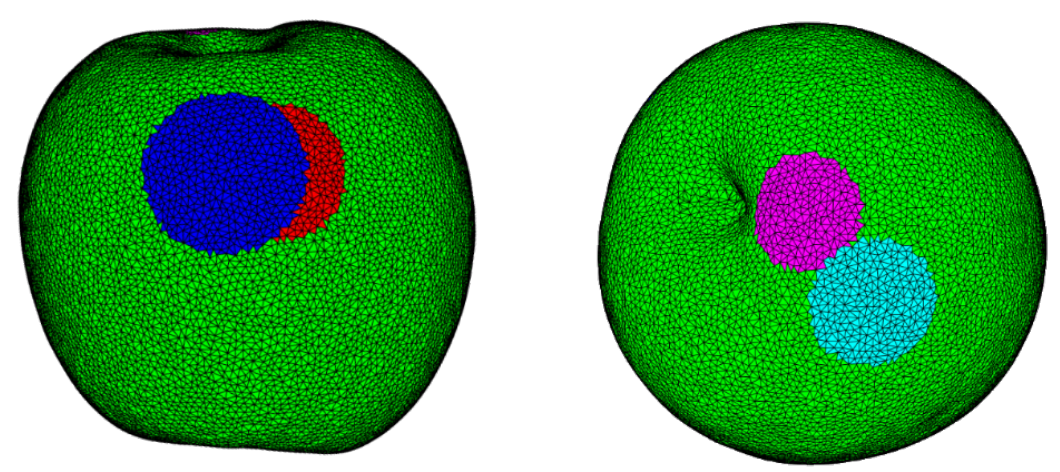

Figure 5.9: Depiction of the facet-wise bruise overlap implementation 
The accuracy of the method was tested through experiments where apple specimens were struck with the spherical impactor mentioned in Chapter 3.2.2. Each specimen was struck twice with overlap occurring on half of the original BA and the force of each impact recorded. The resulting damage was measured as specified in Chapter 4. The bruise damage predicted through the numerical correlations in Chapter 4.6 were used to obtain the bruise data corresponding to each of the measured impacts. This bruise data corresponding to the measured impacts was subsequently used for testing the calculation method of the overlap formulation. Comparisons between the bruise damage measured experimentally and predicted numerically is given in Figure 5.10 for the BV, Figure B.9 for the BA and Figure B.10 for the BD. The tests were repeated nine times for different impacting scenarios with either, two impacts of approximately the same force (60$60 \mathrm{~N})$, one where the second force was greater $(60-120,30-110 \mathrm{~N})$ and one where the first force was greater $(130-60,120-30 \mathrm{~N})$. The comparisons show a close agreement between the measured bruise damage and what was predicted numerically.

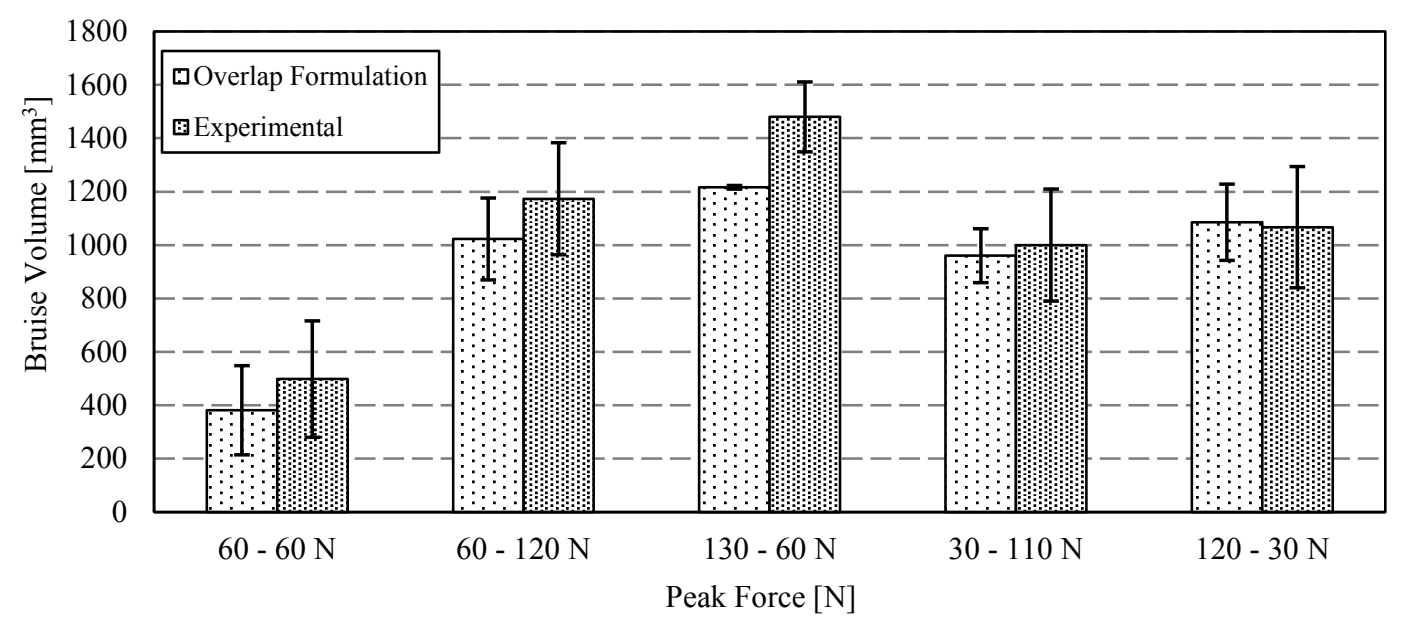

Figure 5.10: Comparison between the overlap formulation and experimental overlapping bruises for the resulting BV. Errors bars indicate 1 SD for 9 repetitions

The use of the facet-wise bruise overlap implementation for a large number of bruises was validated and is presented in Chapter 6. Good agreement between the bruise damage predicted by the overlap formation and that measured from the experiments was achieved. An illustration of the multiple bruises displayed from the post-process formulation is given in Figure D.4 in Appendix D. 


\section{EXPERIMENTAL VALIDATION OF THE DEM BRUISING MODEL}

\section{EXPERIMENTAL VALIDATION OF THE DEM BRUISING MODEL}

When constructing a predictive model it is of importance to access and reckon the predictive capability of the derived model. This provides a degree of confidence when the DEM model is used to predict a situation encountered in practice. It is customary for initial assessments to make use of a visual, qualitative comparison followed by a quantitative comparison between the simulation and the experimental results.

\subsection{Validation Procedure}

For the validation of the DEM model, capable of predicting apple damage during handling, four experiments were performed, each increasing in complexity. Bruise damage served as the primary means of model validation. The three mechanisms by which bruise damage was quantified are those discussed in Chapter 4 namely, bruise volume, bruise area and maximum bruise depth. To this end the first experiment consisted of a simple drop test, the second made use of a vibrating cylinder with a single apple placed inside or a couple of apples with one fixed, the third involved testing apples within a rotating drum and the fourth vibrated specimens in a box. Qualitative visual comparisons were significant for the last two experiments with bruise damage assessments being significant throughout.

The particles were given a uniform random size distribution between a minimum diameter of $55 \mathrm{~mm}$ to a maximum diameter of $76 \mathrm{~mm}$, which corresponded to the minimum and maximum apple diameters measured during the experiments. The only outstanding particle attribute was the material density that was required to compute the mass properties and integrate the equations of motion in DEM. The mean density of the test apples was subsequently determined and is detailed in Appendix E. The mean density for the GD apples was found as $778 \mathrm{~kg} / \mathrm{m}^{3}$.

\subsection{Drop Tests}

Model validation commenced with simple drop tests. These served to test the accuracy of the contact model along with the coupling of the peak force, produced by the contact model, and the bruise prediction model. Importantly, no repeated 


\section{EXPERIMENTAL VALIDATION OF THE DEM BRUISING MODEL}

impacts occurred in a single drop test thus model accuracy, without the influence of overlapping damage regions, was tested. This minimised the inevitable bruise measurement inaccuracies that occurred when testing a large sample of apples scattered with single, multiple and overlapping damage regions.

Two contact situations were tested namely, apple-wall and apple-apple. Ten specimens were dropped from various heights onto the same force transducer specified in Chapter 3.2.2 with the resulting damage measured as outlined in Chapter 4.1. The apples were all dropped on their major diameter, as described by Mohsenin (1986:542), whilst for the apple-apple impacts the bottom apple was fixed to allow for impact on its major diameter. For the apple-plate case, the specimen was dropped on the plate impactor described in Chapter 3.2.2. For the apple-apple case, the bottom apple was fixed using the apple impactor described in Chapter 3.2.2, Figure 3.4. The drop test was simulated in $P F C^{3 D}$ for the same heights and repeated for the minimum, maximum and median apple sizes established for the simulations. The results of the peak impact force for the appleplate case are depicted in Figure 6.1.

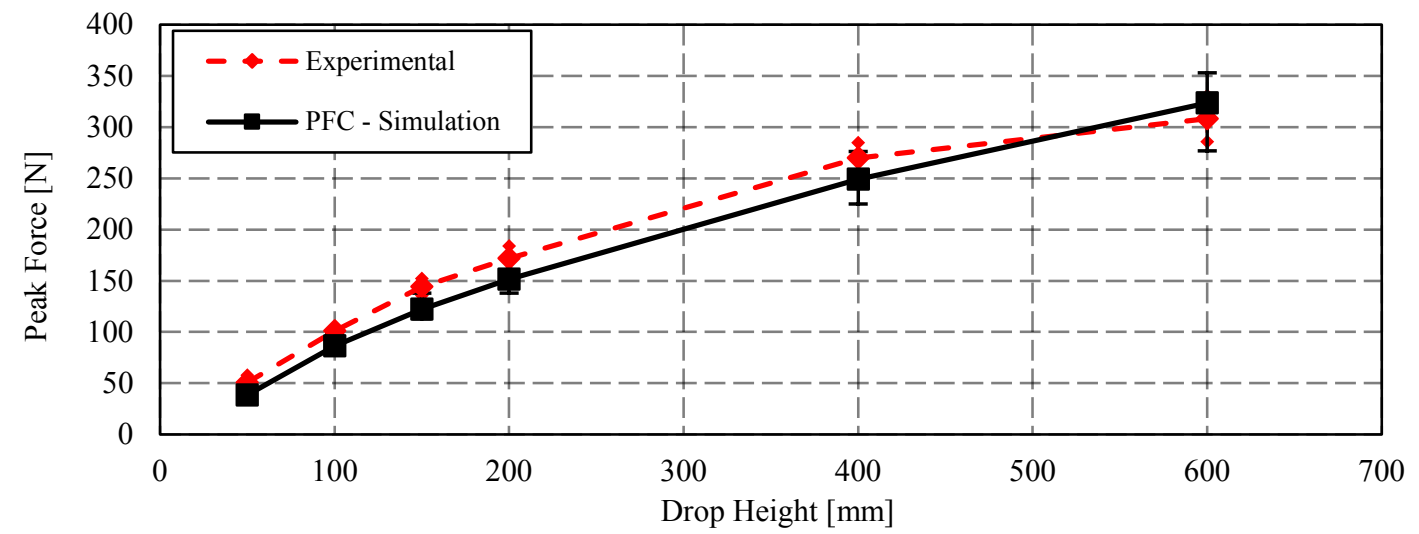

Figure 6.1: Apple-plate drop test comparing peak forces. Error bars indicate 1 SD for the experiment and $\mathrm{min} / \mathrm{max}$ clump size for the simulation

A good agreement between the simulated apple drops and the experiments were achieved. The peak force increased non-linearly with an increase in drop height, very similar to the results obtained by Mohsenin (1986:530). This shows that the contact model was capable of capturing the increase in the damping component of the contact force present as the deformation rate and plastic deformation increased for the increased drop heights. 


\section{EXPERIMENTAL VALIDATION OF THE DEM BRUISING MODEL}

An accurate calculation of the peak contact force also resulted in an accurate prediction of the associated bruise damage for $\mathrm{BV}, \mathrm{BA}$ and $\mathrm{BD}$. The results of the apple-plate drop test for the bruise damage measurements are shown in Figure 6.2. A good agreement between the predicted bruise damage and the experimental results was observed. Consequently, the clump-wall prediction model was cable of accurately predicting apple-plate damage for a single contact situation.
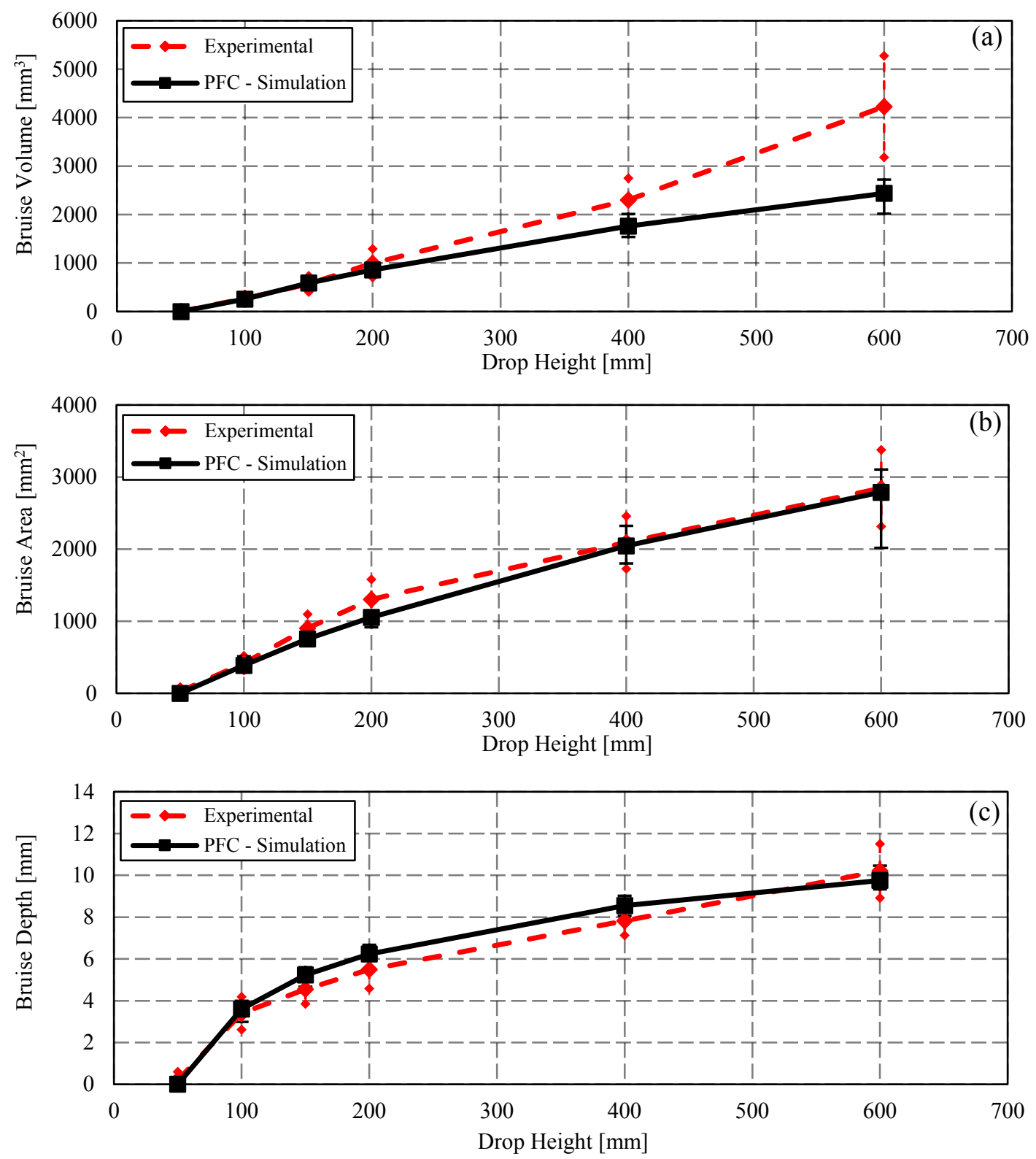

Figure 6.2: Apple-plate drop tests comparing bruise damage, (a) BV, (b) BA and (C) BD. Error bars indicate $1 \mathrm{SD}$ for the experiment and $\mathrm{min} / \mathrm{max}$ clump size for the simulation 


\section{EXPERIMENTAL VALIDATION OF THE DEM BRUISING MODEL}

In the case of the apple-apple drop tests, a good agreement was found between the experimental peak contact force and the predicted contact force, shown in Figure 6.3. Both the experimental and the simulated peak force were shown to be less for the apple-apple case when compared to the apple-wall case. This is in agreement with literature (Mohsenin, 1986:547) and the observations of this study as a higher degree of energy dissipation is present.

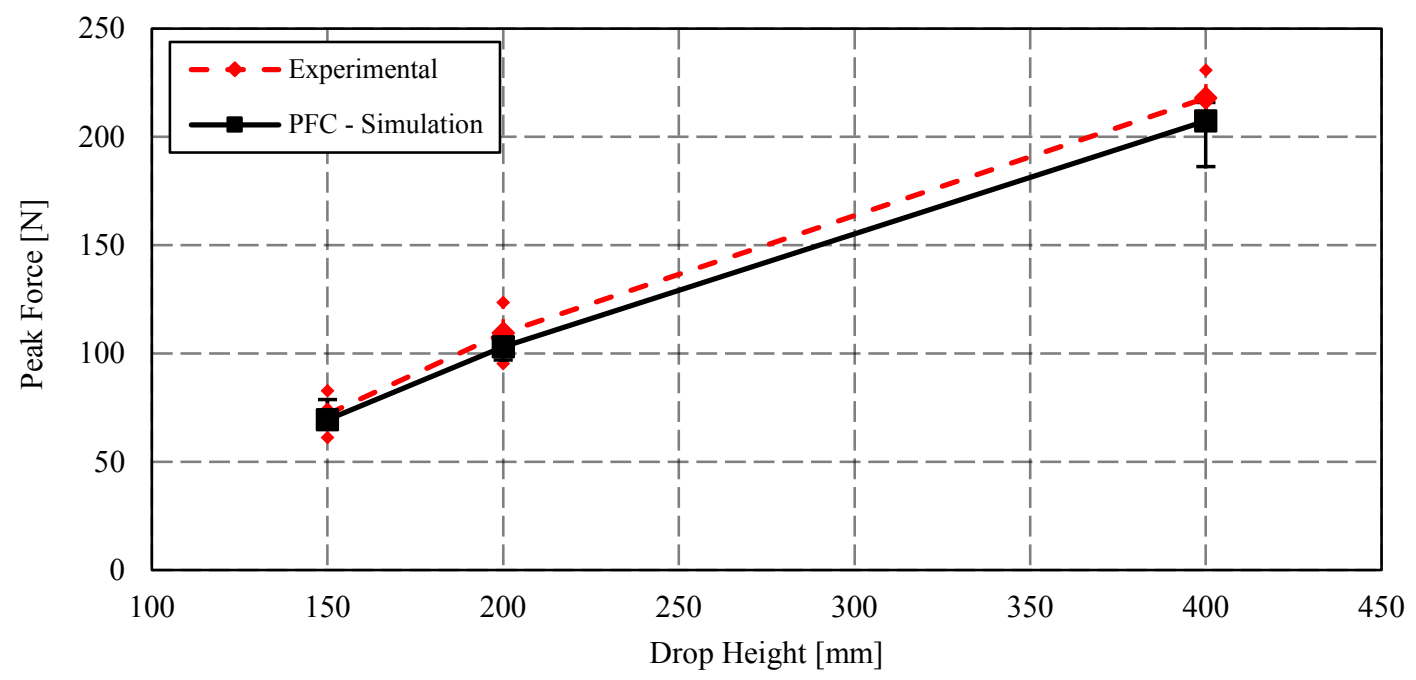

Figure 6.3: Apple-apple drop tests comparing peak force. Error bars indicate 1 $\mathrm{SD}$ for the experiments and $\mathrm{min} / \mathrm{max}$ clump size for the simulations

Bruise damage resulting from apple-apple contact was also found to be in good agreement with that predicted by the simulation. As described in Chapter 4.1 the resulting bruise damage of the falling and stationary apple was summed for comparison between experiment and simulation. The simulation's resulting bruise damage was equal for both the stationary and falling apple as the peak force was equivalent for both apples. The bruise damage predicted by the simulation between the falling and stationary apple was accordingly also summed. The comparison is shown in Figure 6.4. A higher degree of discrepancy between the experimental and simulated apple-apple bruise damage was observed when compared to the apple-plate contacts in Figure 6.2. This is due, in part, to the higher degree of natural biological variability present when two apples are in contact as opposed to only one. Additionally, a greater degree of measurement uncertainty is present when two apples' bruise damage is quantified by the bruise estimation technique. Furthermore, two apples' bruise damage is correlated by the 


\section{EXPERIMENTAL VALIDATION OF THE DEM BRUISING MODEL}

bruise prediction model, leading to a greater degree of uncertainty in the simulation. Good comparisons between the experiment and simulation were however still achieved.
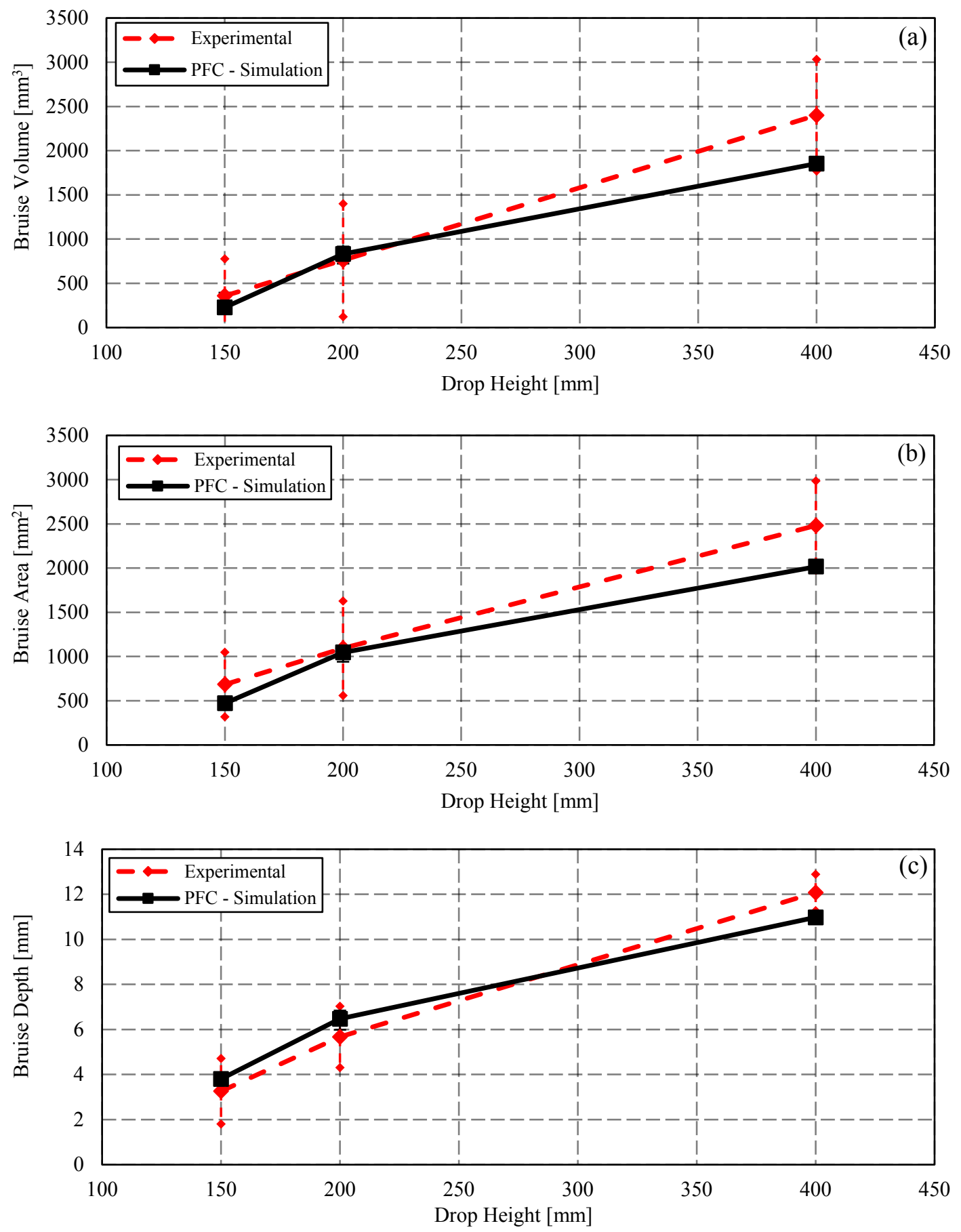

Figure 6.4: Apple- apple drop tests comparing bruise damage, (a) BV, (b) BA and (c) BD. Error bars indicate $1 \mathrm{SD}$ for the experiments and $\mathrm{min} / \mathrm{max}$ clump size for the simulations 


\section{EXPERIMENTAL VALIDATION OF THE DEM BRUISING MODEL}

\subsection{Vibrating Cylinder}

The second validation experiment made use of a vibrating cylinder (Ø $140 \mathrm{~mm}$ ) and a comparison between the developed DEM model and an equivalent experimental set-up. A vibrating cylinder allowed individual specimens to be tested under multiple random impacts. The use of a cylinder, as opposed to a box, minimised the randomness of the wall contacts when comparing experimental and simulation results. The cylinder was vibrated vertically following a sine wave with a frequency of $8.5 \mathrm{~Hz}$ with peak accelerations of approximately $3 \mathrm{~g}$. The specimens were shaken for a period of 10,20 and 30 seconds respectively. Each test was repeated three times and the bruise damage recorded as specified in Chapter 4.1. The analysis was first performed for a single apple within the cylinder along with its DEM equivalent. Secondly, the analysis was performed with two apples with the bottom apple fixed to the cylinder's base using a lag bolt. A depiction of the experiment and the simulation is provided in Figure 6.5
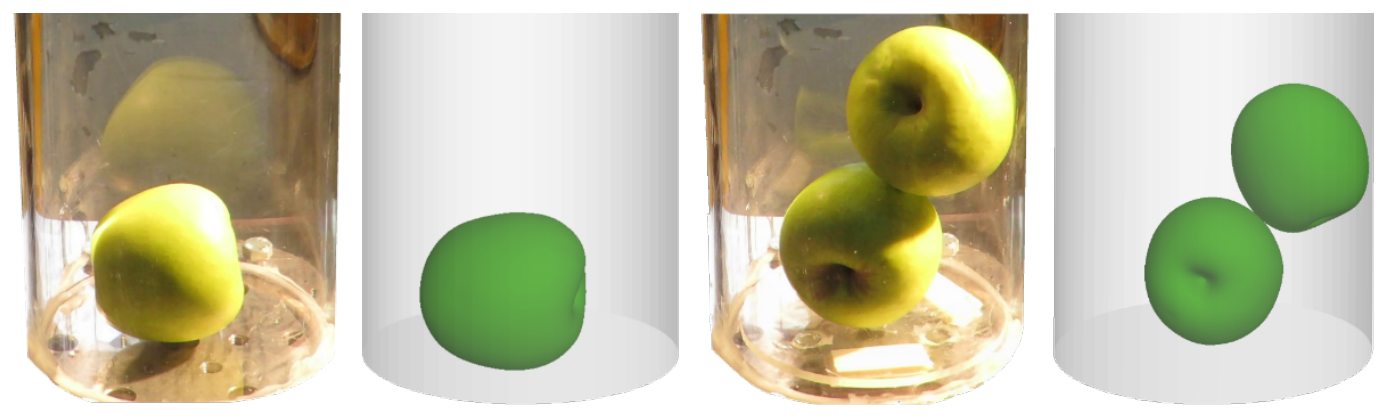

Figure 6.5: Experimental set-up and DEM model for the vibrating cylinder

The resulting $\mathrm{BV}, \mathrm{BA}$ and maximum $\mathrm{BD}$ for the single apple is depicted in Figure 6.6. An acceptable agreement is observed. Very good results were found at the shortest run time of 10 seconds whilst a run time of 20 seconds also produced simulation results close to that of the experiment. With the run time increasing, the amount of randomness in both the experiment and simulation increased. Accordingly, a larger difference between the amount of damage occurring due to single bruises or overlapping bruises would occur. Consequently, the deviation between the experimental and simulation results would increase. However, both the experimental and simulation results still correlated well. 
EXPERIMENTAL VALIDATION OF THE DEM BRUISING MODEL
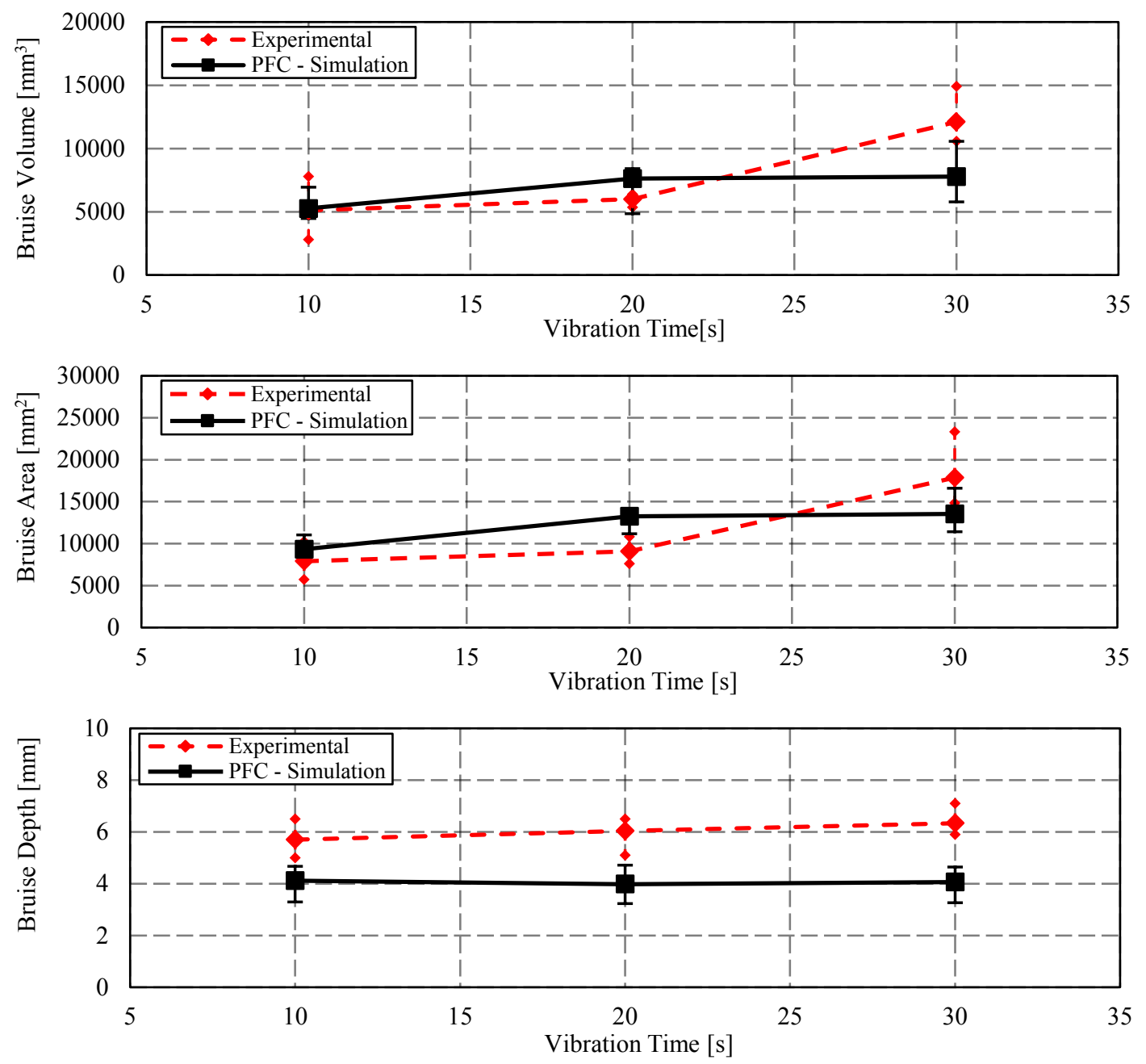

Figure 6.6: Comparison between the experiment and simulation of the vibrating cylinder with a single apple, (a) BV, (b) BA and (c) BD. Error bars indicate $\mathrm{min} / \mathrm{max}$ for the experiments and $\mathrm{min} / \mathrm{max}$ clump size for the simulations

The results for the vibrating cylinder with two apples, with the bottom one fixed to the cylinder, are given in Figure 6.7. Acceptable agreement at the shortest run time of 10 seconds is observed. For larger run times the effect of the randomness of the impact locations and the difference between single impacts, multiple impacts and overlapping impacts was compounded when two apples were involved. This inevitably led to larger differences in both the measured and simulation results. Furthermore, a higher degree of uncertainty is present when two apples are involved in contact, as discussed in Section 6.2, thus larger differences between the simulation and experiment for the longer run times would be expected. The simulation was still cable of predicting BV's, BA's and maximum BD's that were less than those found for the single apple (both experimentally and numerically). 
EXPERIMENTAL VALIDATION OF THE DEM BRUISING MODEL
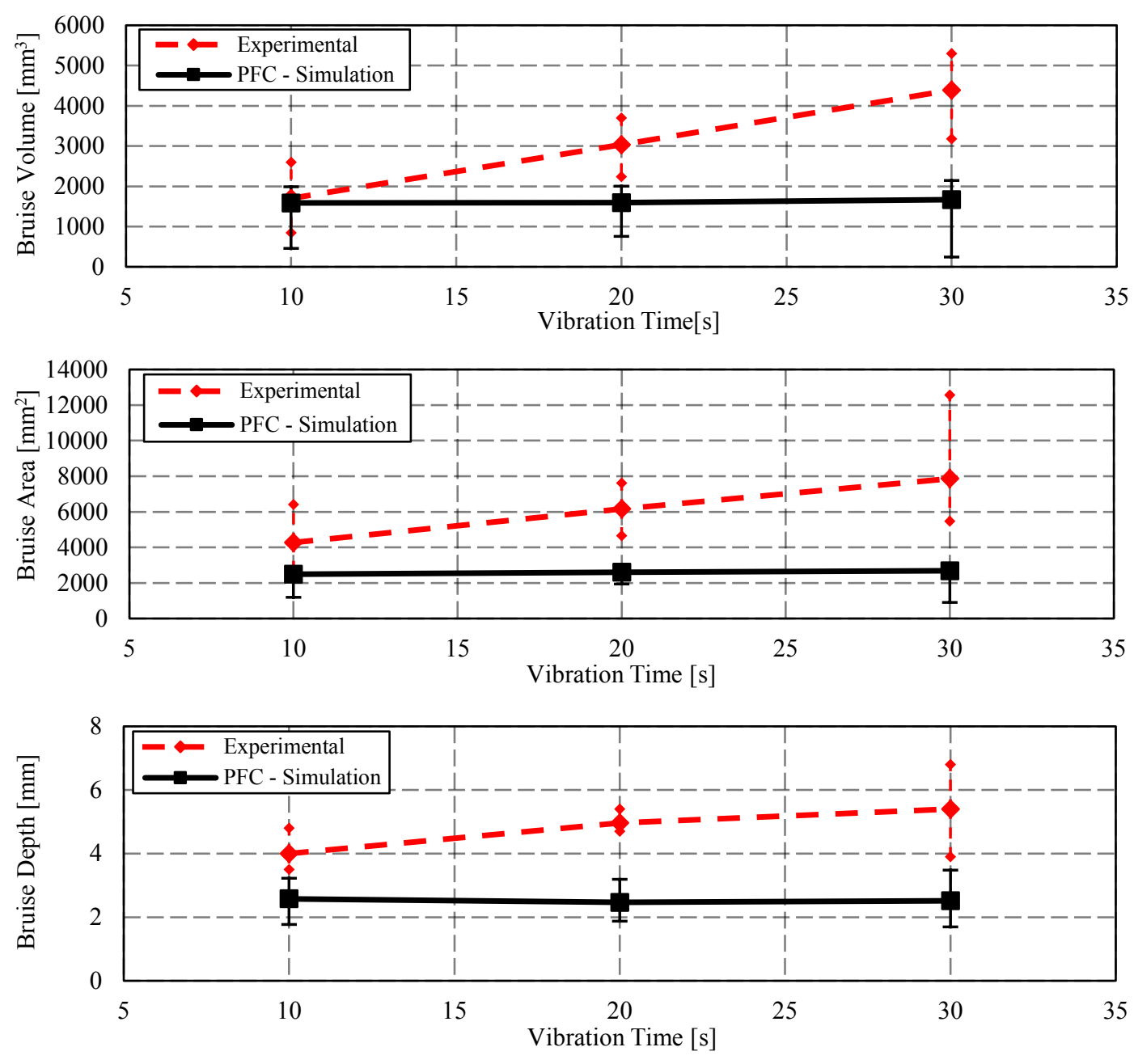

Figure 6.7: Comparison between the experiment and simulation of the vibrating cylinder with two apples, (a) BV, (b) BA and (c) BD. Error bars indicate min/max for the experiments and $\mathrm{min} / \mathrm{max}$ clump size for the simulations

\subsection{Rotating Drum}

The third validation experiment involved the use of a rotating drum typically used in the DEM field to analyse the dynamics of granular flows (Coetzee, 2017., Yang et al., 2017., Sarracino et al., 2004., Mellmann, 2001. \& Höhner et al., 2013). The use of the drum allowed for a relatively large number of particles to easily be simulated and the conditions between the experiment and simulation to be readily matched. A similar drum of smaller dimensions was used in a previous investigation into apple bruising, but no DEM modelling was conducted (Kuang, 1998:31). Furthermore, the depth of the drum could be varied to control whether particle-particle or particle-wall contacts were dominant. The rotation speed of the 


\section{EXPERIMENTAL VALIDATION OF THE DEM BRUISING MODEL}

drum could also easily be changed to allow the impact forces to be altered. Consequently, good qualitative visual comparisons between the experiment and the simulation could be produced.

Two different scenarios were analysed involving the rotating drum. For the first, the drum's depth was set at $200 \mathrm{~mm}$ and for the second the depth was set at $400 \mathrm{~mm}$. The drum had a diameter of $1.0 \mathrm{~m}$ and six equally spaced semi-circular lifters, each with a radius of $50 \mathrm{~mm}$, which prevented sliding motion. Both experiments made use of 50 specimens. Accordingly, the number of apple-apple contacts was greater for the shallower depth and the deeper depth had a larger number of apple-wall contacts. The rotation of the shallower drum was set at $3.5 \mathrm{rpm}$ whilst the deeper drum was set at $4 \mathrm{rpm}$. Both scenarios were run for two and a half rotations of the drum. The qualitative comparison for the $200 \mathrm{~mm}$ drum is given in Figure 6.8. Additionally, the simulation was performed comprising only simple spheres (balls) with the same size distribution as the apples. The comparison between the balls and the more detailed shape approximation of the clumps (discussed in Chapter 5.2) allowed for the importance of an accurate shape approximation to be evaluated. Especially since crude simulations, where the shapes are only represented by spheres, allow for much faster computation times.

An excellent qualitative agreement between the simulation and experiment is observed in Figure 6.8. From Figure 6.8a, the fill height reached by both the simple ball simulation and that of the clumps and the experiment was equal. Within Figure $6.8 \mathrm{~b}$, the height which the clumps and the experiment reached on the side of the drum was equal but the balls tended to roll over one another and struggled to reach the column height of the experiment. The detailed approximation of the apples by the clumps captured the stacking observed during the experiments. This was due to the concave shape of the apple's top and bottom. The simple spheres could not mimic this behaviour as they tended to roll over one another. Consequently, the breakaway angle that was observed for the experiment and the clump simulation, in Figure 6.8c, was equal but could not be replicated by the balls. In Figure $6.8 \mathrm{~d}$, a higher degree of cascading is observed for both the clump simulation and experiment where the balls are shown to roll over one another. Consequently, a superior comparison for the simulation involving the clumps was 


\section{EXPERIMENTAL VALIDATION OF THE DEM BRUISING MODEL}

achieved with the true behaviour and dynamics of the experiment being closely replicated by the simulation.
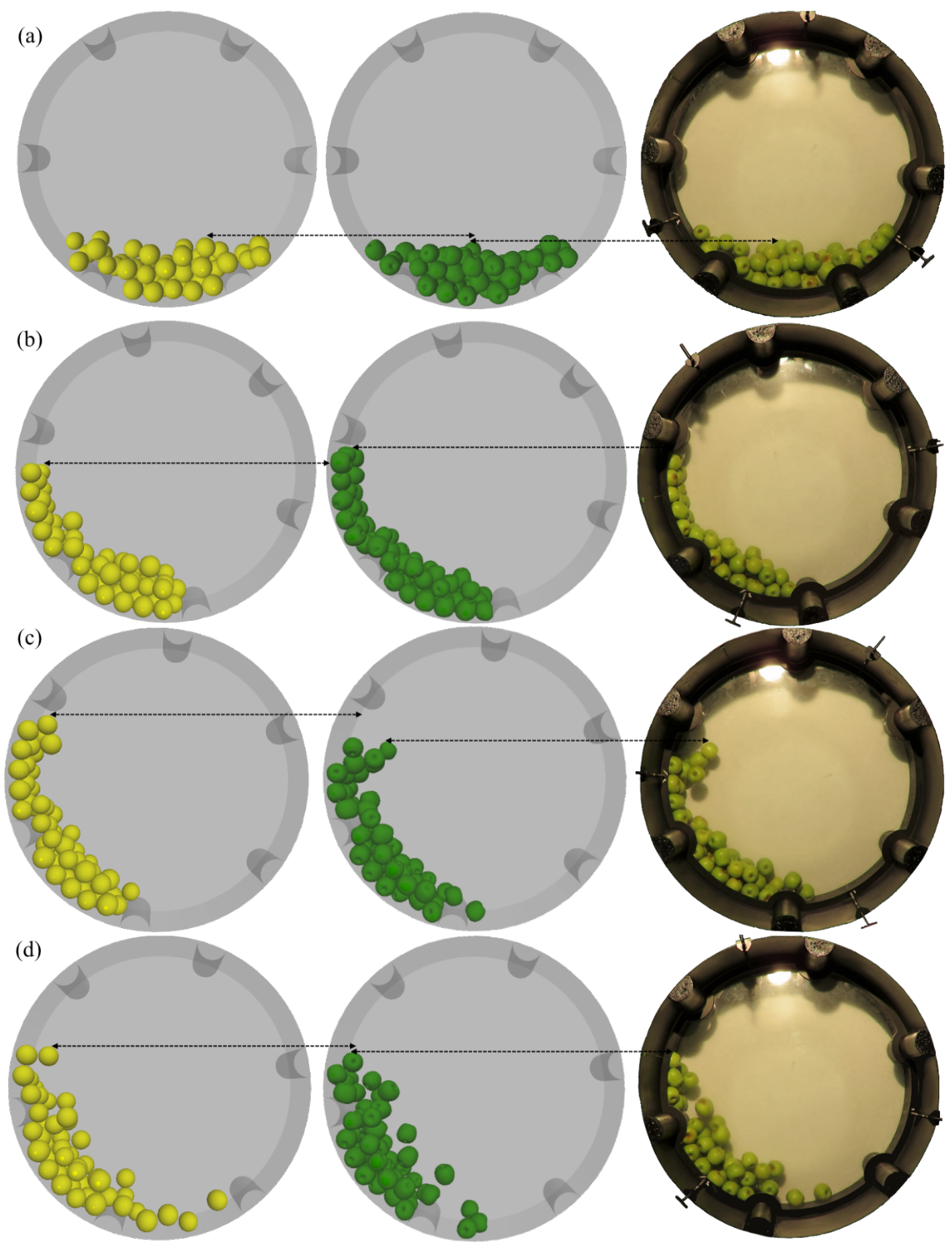

Figure 6.8: Visual comparison between simulation and experiment with the rotating drum set at $200 \mathrm{~mm}$ depth, with spheres on the left, clumps in the centre and the experiment on the right 
EXPERIMENTAL VALIDATION OF THE DEM BRUISING MODEL
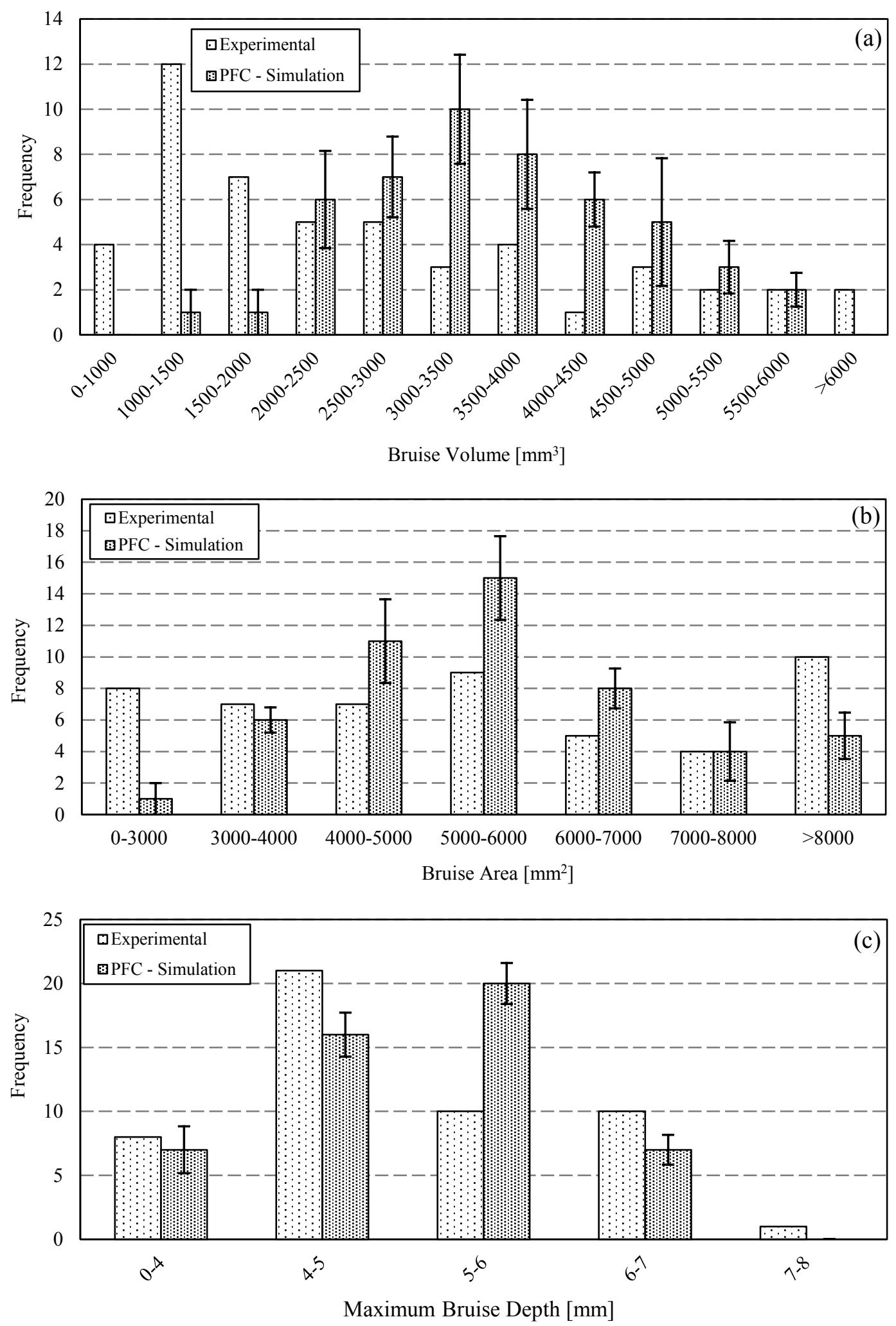

Figure 6.9: Comparison between simulation and experiment with the rotating drum set at $200 \mathrm{~mm}$ depth, (a) BV, (b) BA and (c) maximum BD respectively.

Error bars indicate 1 SD for 5 simulation repetitions 


\section{EXPERIMENTAL VALIDATION OF THE DEM BRUISING MODEL}

The resulting bruise damage comparison is illustrated in Figure 6.9. A comparison between the total amount of BV, BA and maximum BD, present per apple, in the experiment and that predicted by the simulation was made. The amount of bruise damage present, for the $\mathrm{BV}, \mathrm{BA}$ and $\mathrm{BD}$, was divided into different classifications. The frequency at which the amount of bruise damage fell into one of the classes for the experiment and simulation for the 50 specimens was recorded.

A reasonable match was achieved between the simulation and experiment. Similar bruise damage distributions were observed for the BV, BA and maximum BD. The simulations' distribution tended to be slightly more in the shape of a natural distribution, most likely due to the use of the mean contact parameters established in Chapter 3. Additionally, from Chapter 4.3, it was discussed that apple-apple bruise damage was distributed at random between two impacting specimens. The DEM model assigned the same amount of total bruise damage but distributed it equally between two clumps in contact. This also reduced the allocations of the smallest and largest amounts of bruising possible with a more medial amount allocated between two clumps in contact. Consequently, the DEM model was capable of accurately predicting bruising damage in a simulation with a realistic number of particles/apples.

For the second scenario, with the rotating drum's depth set at $400 \mathrm{~mm}$ the qualitative comparison is given in Figure F.2 in Appendix F. The same qualitative agreement observed for the drum set at a depth of $200 \mathrm{~mm}$ was achieved. The bruise damage resulting from the drum set at $400 \mathrm{~mm}$ is given in Figure 6.10. A good agreement between the simulation and experiment is observed. The simulation did however predict bruise damage that was slightly larger than the experiment for the $400 \mathrm{~mm}$ depth case. The bruise damage resulting from the rotating drum set at a deeper depth did however result in a rather extreme case of apple damage both experimentally and numerically. This can be detected when comparing the bruise damage present for the drum set at a depth of $200 \mathrm{~mm}$ and the bruise damage present for the vibration box in the subsequent section. The larger degree of damage present also led to a larger amount of experimental bruising to be quantified and a larger amount of overlapping bruises in the simulation. Van Zeebroeck et al. (2006:89) also found this problem of ambiguous measurement methods with regards to large bruise surface areas. 
EXPERIMENTAL VALIDATION OF THE DEM BRUISING MODEL
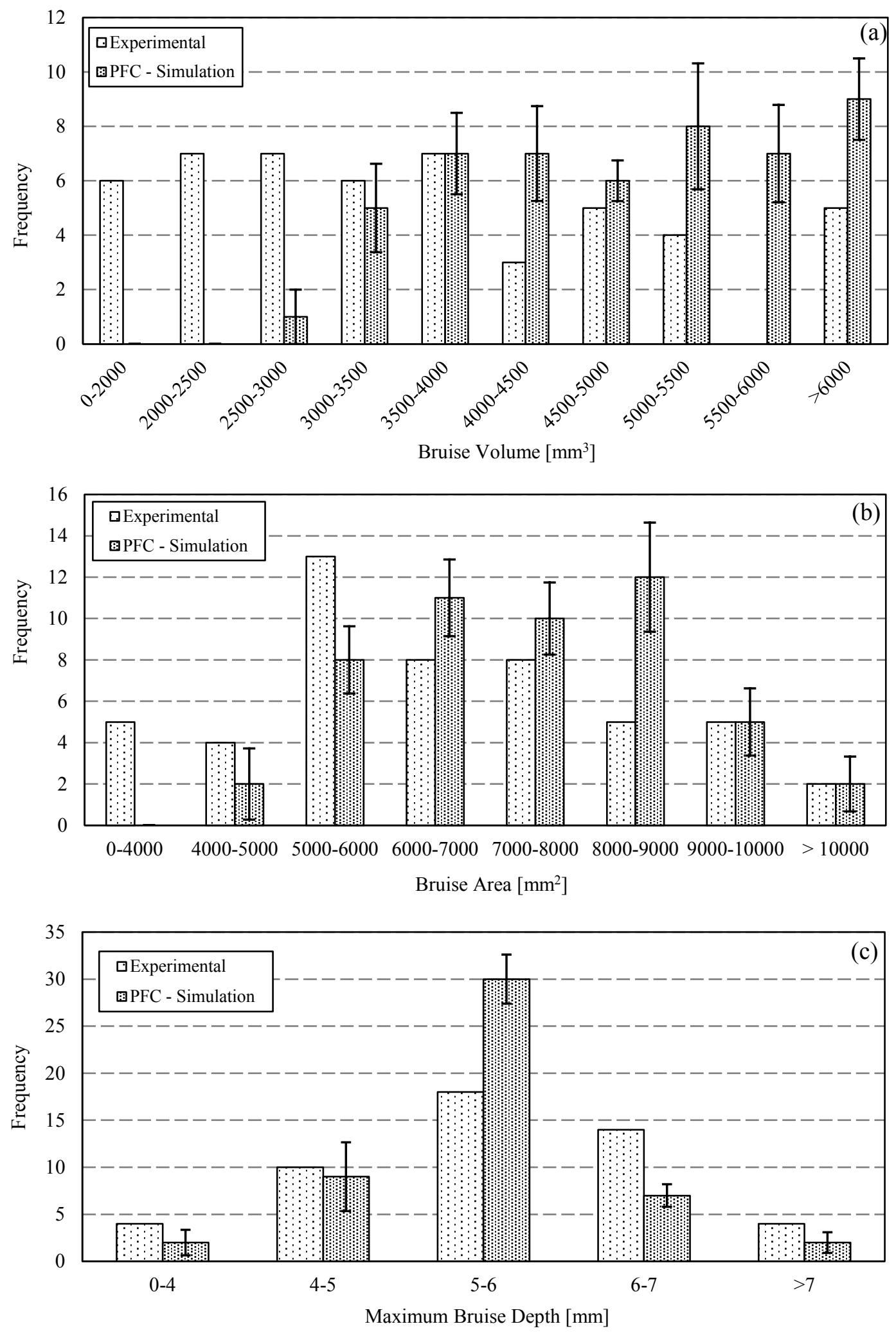

Figure 6.10: Comparison between simulation and experiment with the rotating drum set at $400 \mathrm{~mm}$ depth, (a) BV, (b) BA and (c) maximum BD respectively. Error bars indicate 1 SD for 5 simulation repetitions 


\section{EXPERIMENTAL VALIDATION OF THE DEM BRUISING MODEL}

Consequently, a larger degree of bruise uncertainty is present for both the experimental bruise estimation technique and the simulation's overlap consideration scheme. However, the DEM model still predicted the amount of bruise damage present for the rotating drum set at $400 \mathrm{~mm}$ quite well.

The amount of bruise damage present for the rotating drum's two states was further quantified. To this end, the accuracy of the DEM model's capability of predicting apple damage was identified. Accordingly, the average bruise damage per apple of the experiments and simulations was compared. This gave a good indication of the difference between the simulation and the experiment. The results of the mean differences in bruise damage are given in Table 6.1.

Table 6.1: Quantitative comparison of the bruise damage of the rotating drum

\begin{tabular}{|c|c|c|c|}
\hline Bruise Damage & Experiment $[\bar{x}]$ & Simulation $[\bar{x}]$ & \% Difference \\
\hline $200 \mathrm{~mm}$ & & & \\
\hline $\mathrm{BV}\left[\mathrm{mm}^{3}\right]$ & 2728 & 3583 & $31 \%$ \\
\hline $\mathrm{BA}\left[\mathrm{mm}^{2}\right]$ & 5837 & 5518 & $-5.5 \%$ \\
\hline $\begin{array}{l}\mathrm{BD}[\mathrm{mm}] \\
\mathbf{4 0 0} \mathbf{~ m m}\end{array}$ & 4.9 & 5.1 & $4.1 \%$ \\
\hline $\mathrm{BV}\left[\mathrm{mm}^{3}\right]$ & 3725 & 4785 & $28 \%$ \\
\hline $\mathrm{BA}\left[\mathrm{mm}^{2}\right]$ & 6775 & 7369 & $8.8 \%$ \\
\hline $\mathrm{BD}[\mathrm{mm}]$ & 5.6 & 5.5 & $-1.8 \%$ \\
\hline
\end{tabular}

It is observed that no significant difference was present for the BA and BD with the rotating drum set at $200 \mathrm{~mm}$ depth. The BV predicted by the simulation was fairly larger than the experiment. For the $400 \mathrm{~mm}$ depth case, no significant difference for the BD measurement was observed. The BA was shown to be slightly larger for the simulation whilst the BV was also fairly larger. The close agreement of the BD indicates that the impact force magnitudes predicted by the simulation were accurate. The higher BV's and BA's predicted by the simulation could be attributed to the higher degree of uncertainty present when the BV's and BA's were quantified experimentally. This is reflected by the number of measurements required for BV (four) and BA (two). Consequently, more assessments were required for the BV and BA. Additionally, the presence of multiple overlapping impacts made it problematic to identify the bruise's boundaries. It can be concluded that the DEM model is capable of accurately reflecting the amount of bruise damage present in a simulation with an over-prediction, in the most extreme case, of $31 \%$ for the BV, $8.8 \%$ for the BA and $4.1 \%$ for the BD. 


\section{EXPERIMENTAL VALIDATION OF THE DEM BRUISING MODEL}

\subsection{Vibration Box}

The last validation experiment made use of a vibration box $(0.386 \times 0.282 \times 0.27 \mathrm{~m})$. A vibration box allows for plenty of randomness in terms of apple size, position of contacts, types of contact, number of contacts and simultaneous contacts to be simulated. The initial conditions between the experiment and simulation could also be readily replicated. Accordingly, a shaking box with a vertical sinusoidal displacement was simulated. A structured and random packing fill was compared. The qualitative comparison of the structured packing is given in Figure 6.11.

(a)
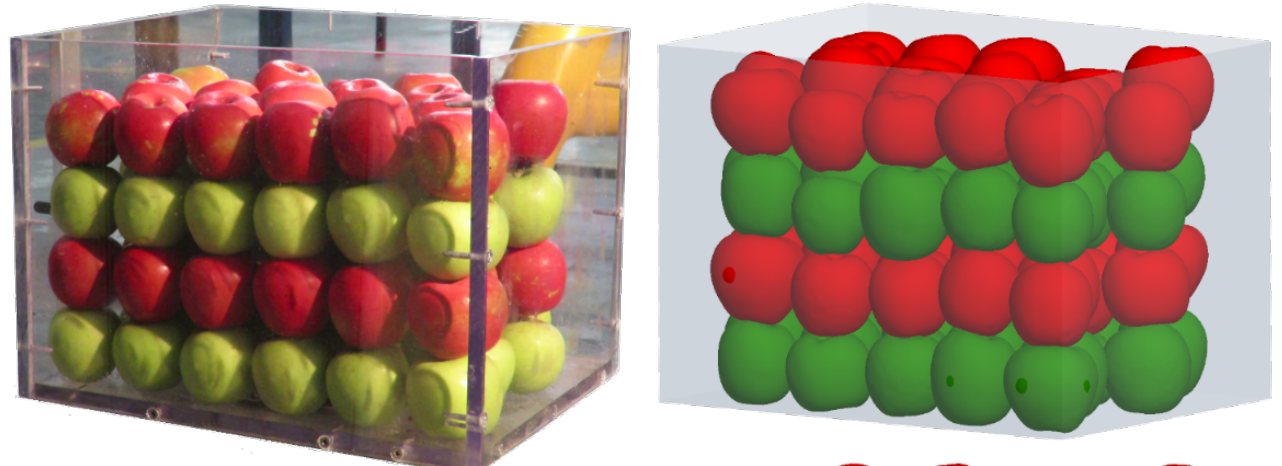

(b)
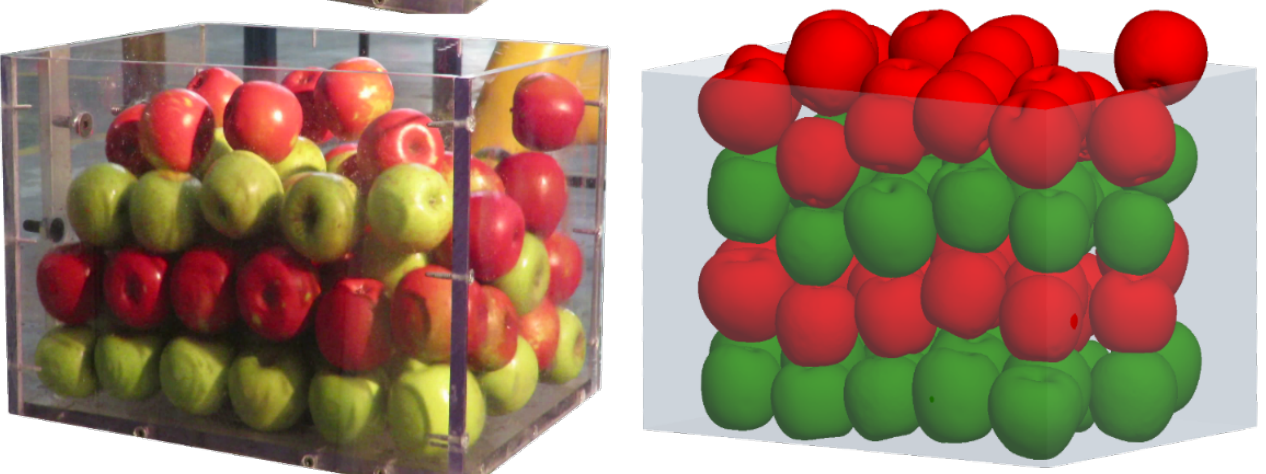

(c)
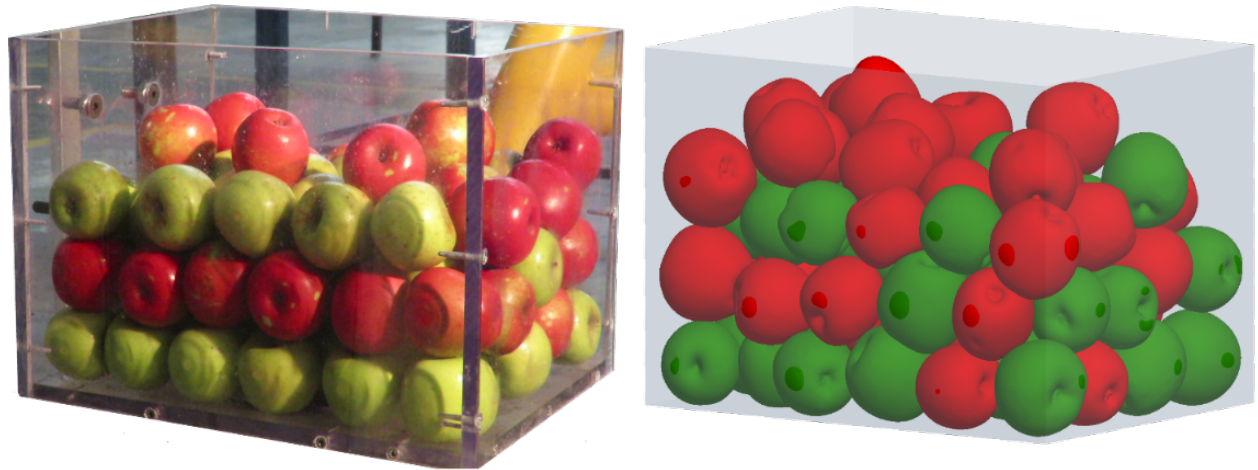

Figure 6.11: Qualitative comparison between simulation and experiment with structured packing in the vibration box, (a) at the start, (b) after 30 seconds and (c) after 60 seconds 


\section{EXPERIMENTAL VALIDATION OF THE DEM BRUISING MODEL}

For the two cases a vibration signal of $8.5 \mathrm{~Hz}$ with a $3 \mathrm{~g}$ magnitude was applied in the vertical direction for one minute. The structured arrangement made use of 80 specimens in four rows whereas the random packing made use of 90 specimens. The structured arrangement shown in Figure 6.11 was used only for qualitative visual comparison, with alternating layers of apples (layers two and four from the bottom) painted a different colour (red). Subsequently, the bruise damage could not be measured. The extent of the influence of the paint on the apple properties was unknown and could slightly alter the frictional characteristics and consequently the shear contact model. Accordingly, the vibration test comprising the structured packing arrangement was repeated with regular apple specimens for quantitative comparison purposes. Additional illustrations of the structured packing vibration tests with regular test apples are given in Appendix F in Figure F.1

(a)

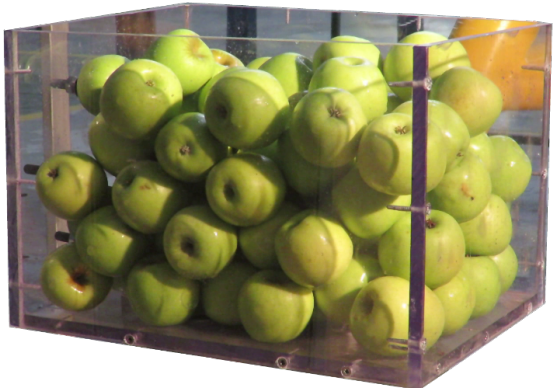

(b)

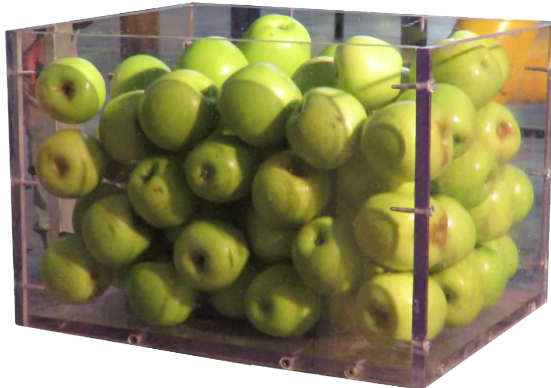

(c)

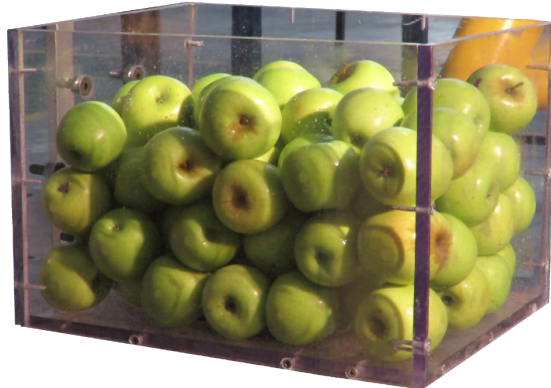

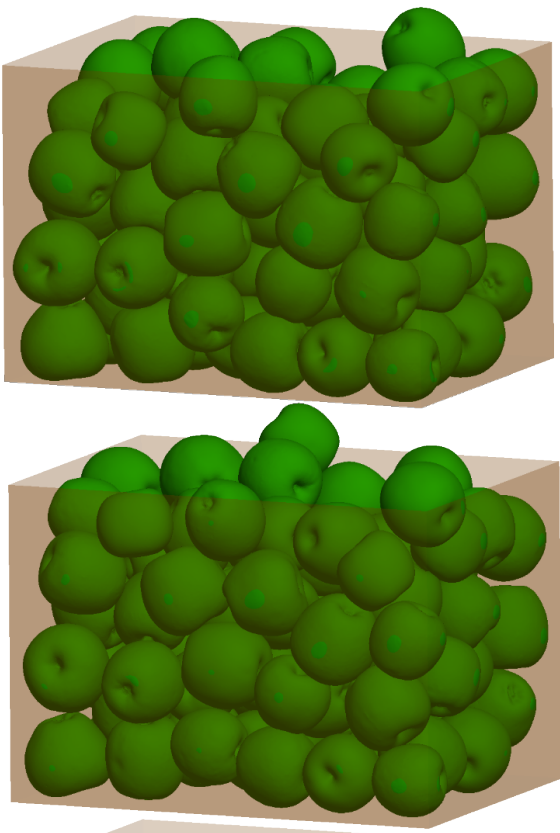

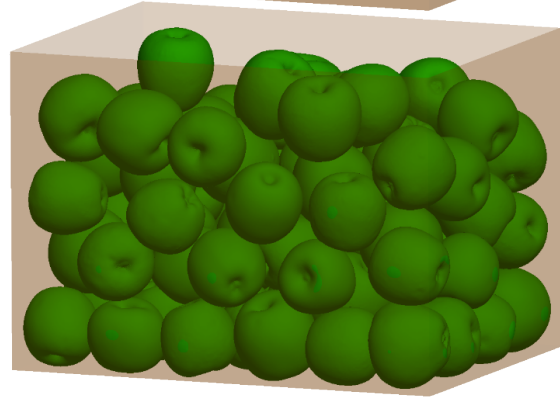

Figure 6.12: Qualitative comparison between simulation and experiment with random packing in the vibration box, (a) at the start, (b) after 30 seconds and (c) after 60 seconds 


\section{EXPERIMENTAL VALIDATION OF THE DEM BRUISING MODEL}

The qualitative visual comparison for the random packing is given in Figure 6.12. A respectable qualitative agreement between the simulation and experiment is observed for both the structured and random case. The resulting bruise damage for the structured case is revealed in Figure 6.13 and the bruise damage for the random case is shown in Figure 6.14.
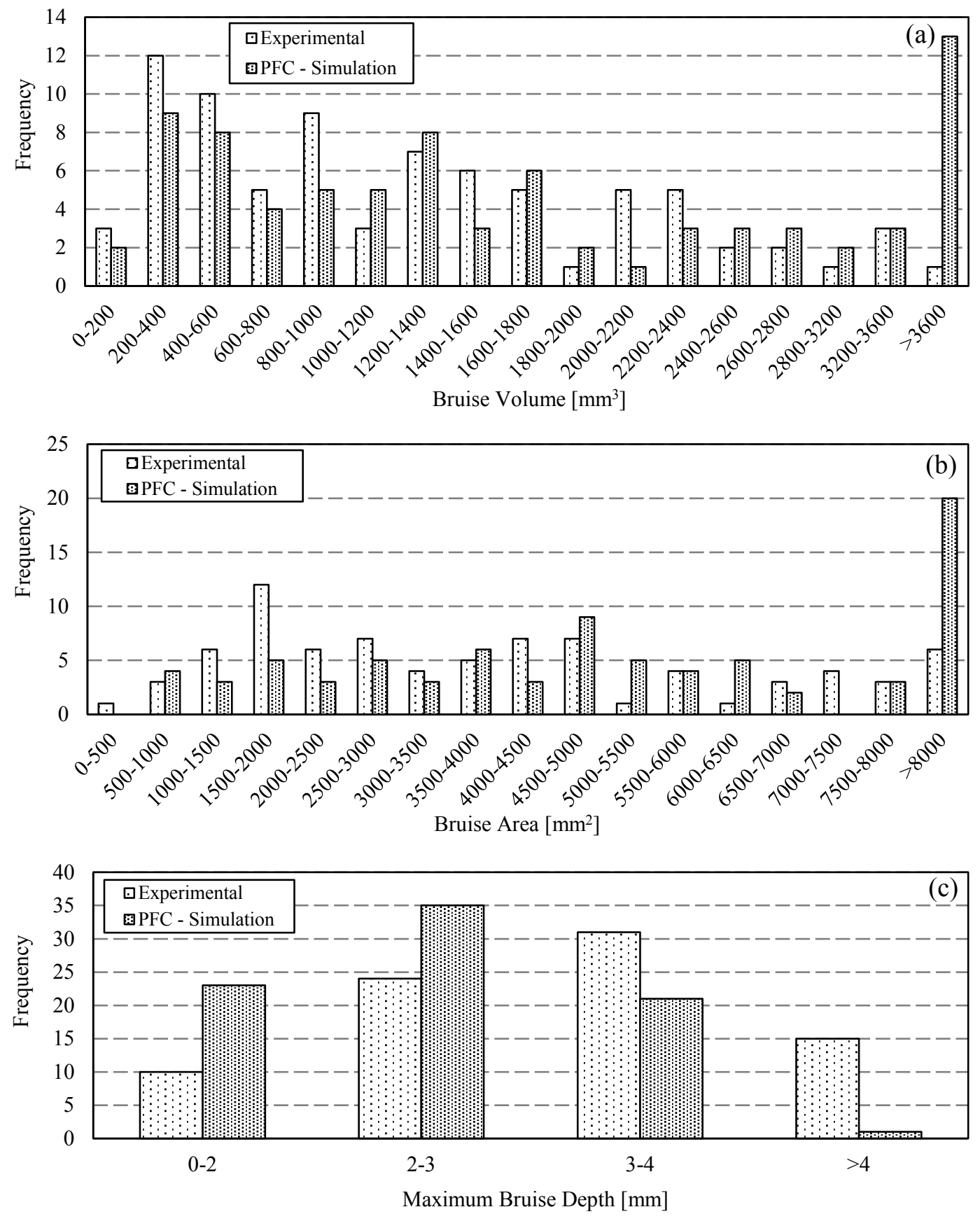

Figure 6.13: Comparison between simulation and experiment for the vibration box with structured packing, (a) BV, (b) BA and (c) maximum BD respectively 
EXPERIMENTAL VALIDATION OF THE DEM BRUISING MODEL
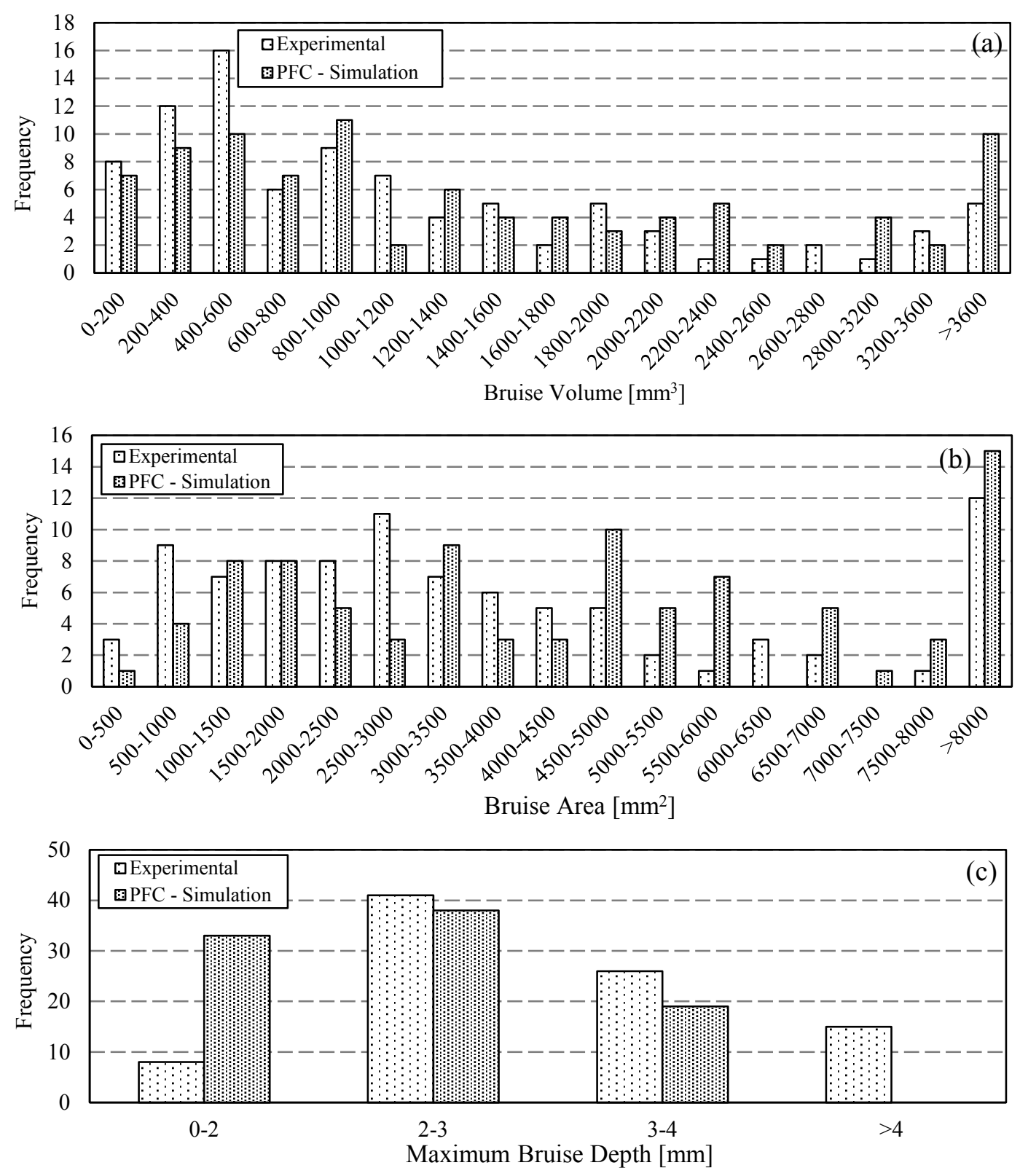

Figure 6.14: Comparison between simulation and experiment for the vibration box with random packing (a) BV, (b) BA and (c) maximum BD respectively

A fairly good match was achieved between the experiment and simulation for the $\mathrm{BV}, \mathrm{BA}$ and maximum $\mathrm{BD}$ in both the structured and random packing case. The minor differences that occurred in the distribution plots of Figure 6.13 and Figure 6.14 may also have been influenced by the simulation's allocation of appleapple damage as discussed in Section 6.4 and measurement uncertainty. Similar distributions of bruise damage were achieved for both the random and experimental case. 


\section{EXPERIMENTAL VALIDATION OF THE DEM BRUISING MODEL}

The damage resulting from the vibration box consisted of bruises that were much shallower than those found in the rotating drum. This is reflected when comparing Figure 6.9 and Figure 6.10 with Figure 6.13 and Figure 6.14. BV's and BD's of half of those present for the rotating drum were found from the vibrating box whilst BA's for the vibrating box were about $65 \%$ the size of those of the rotating drum. No significant difference between the structured and random packing for both the experiment and simulation was observed.

Subsequently, the amount of bruise damage present for the vibrating box's two packing states was quantified and compared with that predicted by the simulation. Accordingly, the average bruise damage per apple in the experiments and simulations was compared. The results of the mean differences in bruise damage are given in Table 6.2.

Table 6.2: Quantitative comparison of the bruise damage of the vibrating box

\begin{tabular}{c|ccc} 
Bruise Damage & Experiment $[\bar{x}]$ & Simulation $[\bar{x}]$ & \% Difference \\
\cline { 1 - 1 } Structured & & & \\
\cline { 1 - 1 } BV [mm $\left.{ }^{3}\right]$ & 1273 & 1869 & $47 \%$ \\
BA [mm²] & 4235 & 5421 & $28 \%$ \\
BD [mm] & 3.5 & 2.5 & $-29 \%$ \\
\cline { 1 - 1 } Random & 1197 & 1626 & \\
\cline { 1 - 1 } BV [mm 3 ] & 3791 & 4697 & $36 \%$ \\
BA [mm²] & 3.0 & 2.4 & $-20 \%$ \\
BD [mm] & & &
\end{tabular}

From Table 6.2 it is evident that the simulation tended to over-predict the BV's and BA's whilst under-predicting the BD's. This could be due to the large degree of randomness involved in the vibration box. Furthermore, the vibrating test set-up made use of a feedback system to supply the specified vibration signal. Consequently, a signal with a slightly higher and slightly lower amplitude may be supplied to the experiment whereas the simulation provided a constant uniform signal. The measurement uncertainty discussed in Section 6.4 could also be involved in the vibrating boxes' experimental results. It can be concluded that the DEM model was capable of predicting the amount of bruise damage present in a simulation with a large number of particles under realistic conditions with an overprediction, in the most extreme case, of $47 \%$ for the BV, $35 \%$ for the BA and an under-prediction $29 \%$ for the BD. 


\section{EXPERIMENTAL VALIDATION OF THE DEM BRUISING MODEL}

\subsection{Bruise Visualisation and Shape Modelling}

The use of $P F C^{3 D}$ for the DEM simulation allowed for the bruising damage to be visualised through the pebbles making up the clumps. Subsequently, a bruise visualisation algorithm was programmed. This allowed for the maximum bruise damage present for a pebble to be recorded and visualised with the use of a colour map. The flow chart for the bruise visualisation is given in Figure D.5 in Appendix D. Accordingly, the bruise position on the clumps could be visualised during simulation runtime along with the bruise distribution in a system of particles. This allows for potential regions responsible for postharvest mechanical damage to be identified during runtime and thus expedites the design process as they do not have to be identified during post-processing.

The bruise distribution in the rotating drum and the vibrating cylinder is presented in Figure 6.15. From Figure 6.15a it is observed that the bruises occurred at random positions on the specimens. This was as expected and is attributed to the vigorous mixing that occurred in the drum. For the vibrating cylinder (Figure 6.15b) the fixed clump is shown to only bruise on the top side of the clump and the loose clumps' bruises were spread randomly across its surface. Exactly the same bruising distribution was observed in the experiment.

(a)

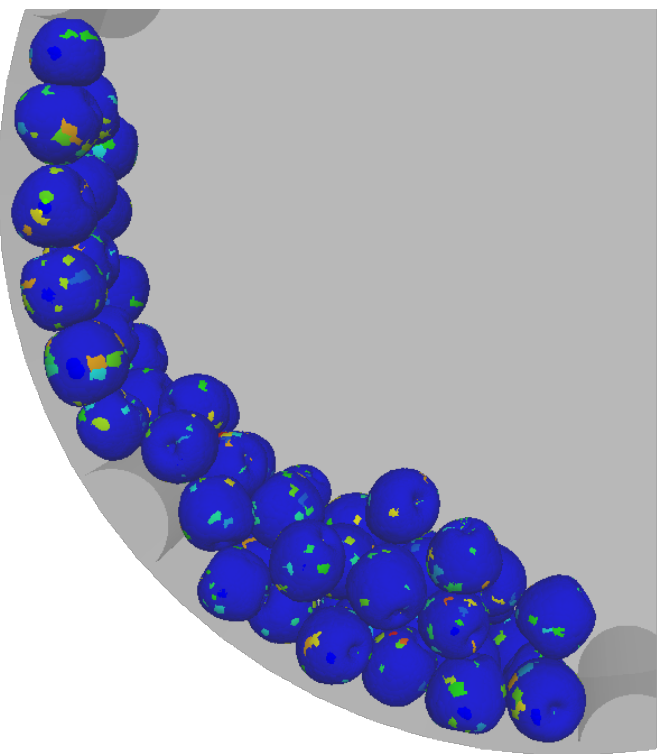

(b)

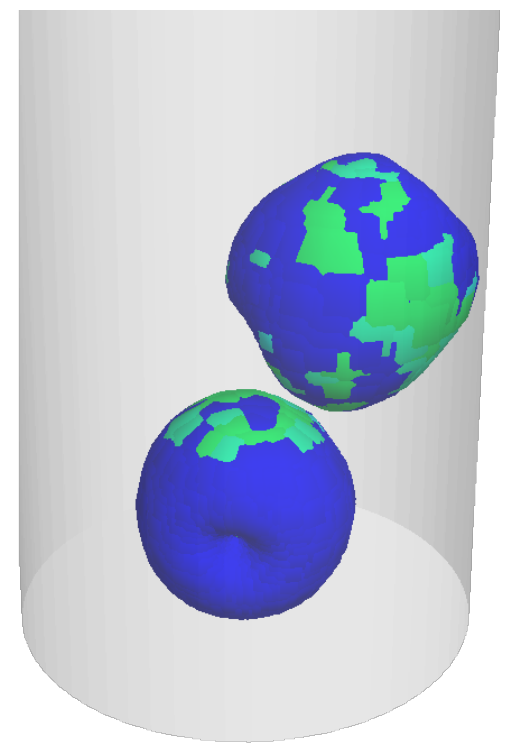

Figure 6.15: Bruise visualisation for the (a) apple drum and (b) vibration cylinder 


\section{EXPERIMENTAL VALIDATION OF THE DEM BRUISING MODEL}

In Figure 6.16 the bruise distribution in the vibration box could be visualised both as bruising occurred throughout the box but also as bruises were distributed across a clump's surface. The use of the bruise visualisation function indicated that the bruise damage increased towards the bottom of the vibration box. A similar trend was witnessed in the experiments.
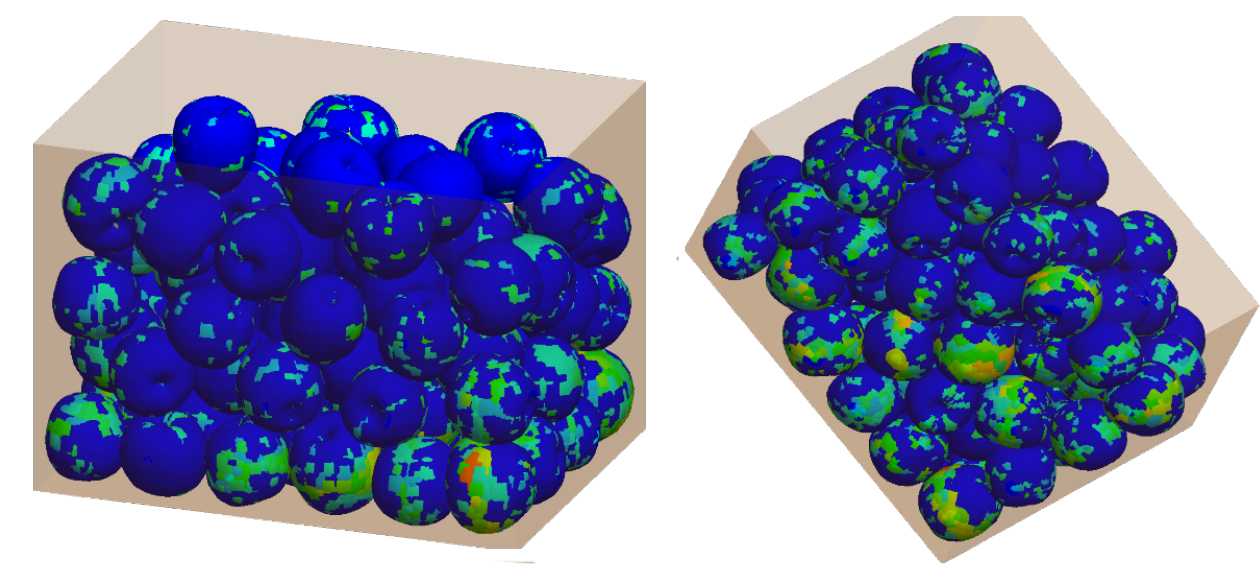

Figure 6.16: Bruise distribution visualisation within the vibration box

For the vibration box it was observed that the apples tend to settle and rotate around their major equatorial axes during extended continuous vibration. Consequently, overlapping bruises were observed to occur in a band around the apple's major axes. A depiction of this occurrence, for an apple from the experiment, is presented in Figure 6.17a. This behaviour was successfully replicated in the model and the bruise visualisation in $P F C^{3 D}$ also showed bruising to occur in a band around the clump's major equatorial axes, as shown in Figure $6.17 \mathrm{~b}$. In the post-processing environment, the full extent of the bruising areas could be visualised and corresponded with the visualisation in $P F C^{3 D}$ and the experiment, as shown in Figure 6.17c.

The observation that apples tend to settle and rotate around their major axis in the vibration box lead to an investigation into the use of single spheres in the vibration box. Accordingly, a simulation with spheres was conducted for the random packing state and compared to the equivalent simulation making use of clumps. It was observed that the single sphere particles (balls) were incapable of replicating this behaviour as the particles did not settle and rotate around only one axis. Figure $6.17 \mathrm{~d}$ shows the bruise distribution across a sphere for the equivalent 


\section{EXPERIMENTAL VALIDATION OF THE DEM BRUISING MODEL}

vibration box simulation with spherical particles. As bruising was shown to occur more randomly for the spheres, the mean rotations of the clumps and the spheres (around the global coordinate system) were compared and are presented in Figure G.2 in Appendix G. It was found that clumps tend to have approximately $20 \%$ fewer rotations than that of the spheres.

(a)

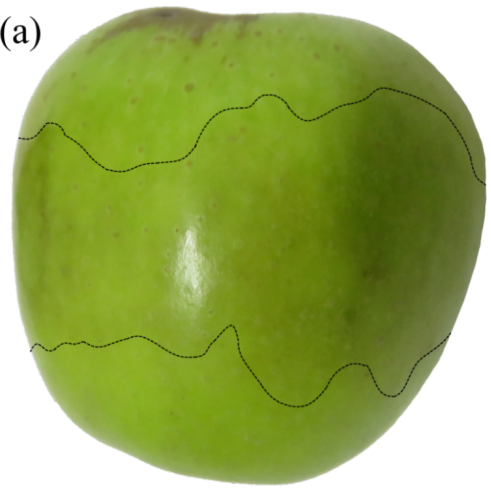

(c)

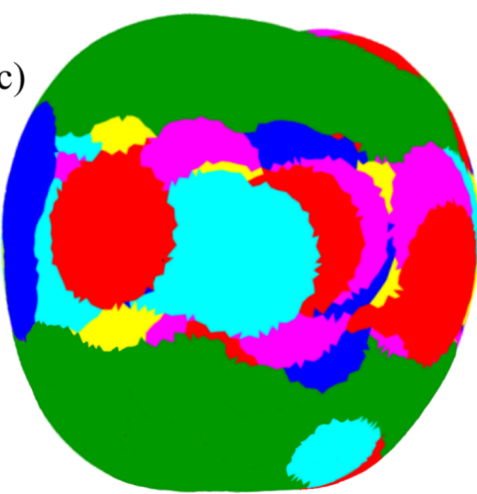

(b)

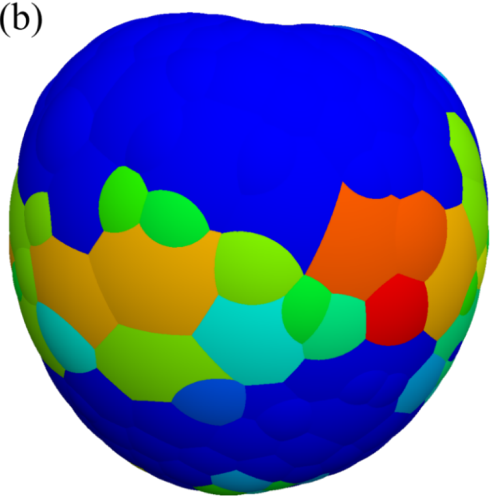

(d)

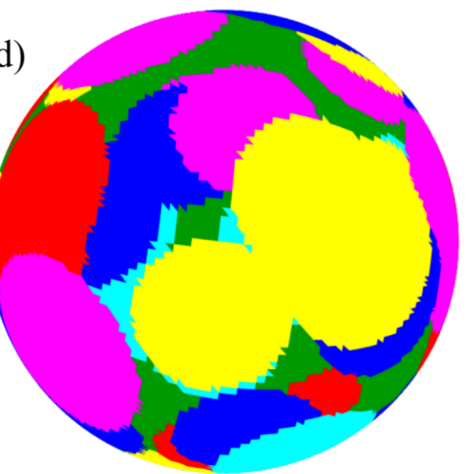

Figure 6.17: Bruise visualisation comparison between spheres and clumps in the vibration box, (a) experimental apple specimen with visible bruise, (b) clump visualisation in $P F C^{3 D}$, (c) clump visualisation in post-processing, (d) sphere visualisation in post-processing

The amount of bruise damage predicated by the spheres was compared to that of the clumps (given in Table 6.2) and is presented in Table G.2 in Appendix G. It was found that spheres greatly over-predicted the bruise damage present in the vibration box with BV over-predicted by $780 \%$, BA over-predicted by $300 \%$ and BD over-predicted by $67 \%$. This over-prediction was attributed to the random scattering of bruises which occurred across the particle surface; this lead to a decrease in the number of overlapping contacts and accordingly the total amount of bruising after accounting for overlap was greater for spheres than for clumps. 


\section{EXPERIMENTAL VALIDATION OF THE DEM BRUISING MODEL}

Consequently, the use of clumps allowed for more accurate replication of the dynamic behaviour of the particles, more accurate contact positioning and more accurate bruise damage results as bruise overlap was accounted for more precisely. Accurate shape modelling proved to influence the accurate prediction of bruise positions. A sensitivity study into the effect of the contact proximity radius (Section 5.4) is presented in Appendix $G$ and found that accurate shape modelling had no effect for the rotating drum but had a large effect for the vibration box. The sensitivity of accurate particle shape representation is normally not known when analysing a system of particles using DEM. The use of clumps provided the most accurate results regardless of the system's sensitivity to particle shape modelling. Consequently, accurate shape representation with the use of clumps is preferable when investigating systems involving fresh produce.

\subsection{Validation Discussion}

The DEM model for predicting apple damage during handling was validated. The model validation was performed with four experiments, each of increasing complexity. A good qualitative and quantitative correlation between the experiment and simulation was obtained. The use of DEM allows for bruise damage to be predicted, processed and quantified in an accurate way where overlapping damage is accounted for. The experimental measurement of a large quantity of bruises resulting from multiple impacts and overlapping impacts is challenging. When considering the accuracy with which the model could predict the apple damage for the experiments, a $47 \%$ over-prediction of the BV was observed, which may seem large but considering a mean quantitative difference of roughly $600 \mathrm{~mm}^{3}$ or $0.6 \mathrm{~cm}^{3}$ illustrates that this difference was actually quite small. This is also true for the BA with a difference of $900 \mathrm{~mm}^{2}$ or $9 \mathrm{~cm}^{2}$ and BD with a difference of roughly $1 \mathrm{~mm}$. The sensitivity of the bruise damage with regards to the elastic moduli and damping constants was investigated and is presented in Appendix G.2. The model was relatively robust regarding large variations in the contact parameters. Considering, the large degree of biological variability, measurement uncertainty, the use of damage estimation techniques and overlapping impacts, it will be difficult to achieve more precise results. Additionally, the use of DEM allows for apple damage to be visualised during runtime. 


\section{CONCLUSION AND RECOMMENDATIONS}

The use of the Discrete Element Method (DEM) to investigate the bruising of fresh produce, outside of its original purpose, was investigated. The production and processing of fresh produce account for a significant proportion of the world's annual production. Mechanical damage during the postharvest handling phase still accounts for a substantial amount of produce losses. One of the most commonly encountered forms of mechanical damage is bruising. Consequently, a DEM model capable of predicting apple damage during handling was successfully developed. The developed model comprised the establishment of an applicable contact force model, development of a bruise prediction model, the numerical implementation of the derived models, accounting for bruise overlap during post-processing and validating the resulting model. Conclusions are subsequently drawn relating to the research objectives along with a discussion of additional future recommendations.

\subsection{Contact Model Outcomes}

A user-defined contact force model that is capable of replicating the behaviour of apple tissue was effectively implemented in the DEM software. A significant amount of literature describing the material parameters needed in order to institute a DEM investigation into fresh produce was found. However, these parameters were measured and obtained out of a purely scientific point of view with little to no attention given to their use in a practical implementation. The corresponding apparent Young's modulus (used in the formulation for the elastic stiffness of the viscoelastic contact force model in this study) was found to be lower at 2.52 3.82 $\mathrm{MPa}$ when compared to the values (3.78 to $7 \mathrm{MPa}$ ) reported in literature. This demonstrated the dependence of the contact parameters on loading rates. Definite calculation of impact forces was accordingly more reliant on the accompanying viscous component in a contact model representative of fluid rich materials like fruit and vegetables. This is even more important when the behaviour of two viscous materials in contact was replicated, as larger and more accurate effective damping parameters were needed when compared to contacts with hard surfaces. Furthermore, the use of mean material properties was sufficient for the efficient implementation of a contact model representative of biological materials. 


\subsection{Bruise Damage Investigation}

A bruise development model competent in describing the formation of bruises resulting from multiple and overlapping impacts was successfully developed. The effect of time between repeated contacts and bruise development was also investigated and found to be of little consequence. Additionally, bruise damage was shown to correspond to the maximum impact force for a range of multiple impact forces. Both these aspects have not been described in literature before. The geometrical shape of the impacting bodies was of great importance when correlating peak force with bruise damage. Bruise damage correlations should be drawn between the exact impacting situations and material conditions expected for the practical situations in question. When apple specimens contacted each other, a completely random allocation of bruising damage was present between the two specimens. However, the total damage shared amongst the two specimen's correlated well with the peak impact force. For the purposes where the damage between two apple specimens needed to be quantifiably predicted, it was acceptable to assume an equal distribution of the total damage present between the bruises.

\subsection{Numerical Implementation and Validation}

The implementation of the bruise damage correlations in the DEM programme was possible with the use of its inherent programming language. Due to simulation time considerations, it was more efficient to account for overlapping bruise damage on a post-process level. Accurate shape (clumps) and contact representation resulted in admirable correlations between experimental bruise damage observations and numerical bruise damage predictions when compared to spherical particles. DEM readily provides the user with contact force information and the dynamic behaviour of a group of particles. The use of a contact model that accurately represented the behaviour of viscoelastic materials allowed DEM to correctly predict the impact forces of viscoelastic materials to within $11 \%$. Coupling between impact forces and damage models allowed for accurate damage predictions to be made in systems involving a large number of dynamic contacts under realistic conditions. DEM could additionally replicate the bulk dynamic behaviour of fresh produce. The position of damage on the fruit could be visually identified as it occurred and 
quantified on a post-process level to within a confidence of roughly $50 \%$ for BV's, $35 \%$ for BA's and $30 \%$ for BD's. In future, evaluations of situations responsible for postharvest damage can readily be identified and eliminated. It is of greater importance to identify and eliminate damage to produce than it is to quantify the resulting damage. However, the developed model has the capability to predict the quantity of damage present to within reasonable levels.

\subsection{Recommendations}

The usefulness of the derived model may be extended by investigating the accuracy with which it can predict postharvest mechanical damage for other fruit and vegetables. Fruit and vegetables whose material properties vary greatly depending on the region of the specimen body could be of interest. The capability of $P F C^{3 D}$ to access the surface coordinates of a contact position, as it occurs, allows for varying properties or even varying contact models to be applied depending on the position of impact. This same capability can be used to alter the material properties if a specimen is impacted on the same position and a significant change in properties resulting from multiple impacts is expected.

\subsection{Conclusion}

The DEM model developed here has the capability for predicting the incidence of apple bruising in the postharvest handling chain. The objectives of this investigation were successfully achieved. The most suitable contact model for DEM simulations indicating mechanical damage to fruit and vegetables was found. The appropriate mechanical parameters for predicting damage relating to apple fruit under dynamic impact conditions were established. Bruise damage correlations were successfully coupled to impact forces, equipping DEM with the capability to predict bruise damage. Numerical models simulating the dynamic mechanical behaviour, contact representations and responses were proficiently implemented. Validation of the developed model was achieved through physical experimentation and comparisons to numerous numerical simulations with good correlations achieved. The accomplishment of the investigation and presentation of the formulated model allows for fluent implementation of the method to other fruit and vegetables. 


\section{REFERENCES}

A growing industry [Agriculture]. 2016. [Online]. Available: http://western cape.top300.co.za/a-growing-industry-agriculture.html [2016, June 17].

Abedi, G. \& Ahmadi, E. 2013. Design and Evaluation a Pendulum Device to Study Postharvest Mechanical Damage in Fruits: Bruise Modelling of Red Delicious Apple. Australian Journal of Crop Science, 7(7):926-968.

Abir, J., Longo, S., Morantz, P. \& Shore, P. 2016. Optimized Estimator for RealTime Dynamic Displacement Measurement using Accelerometers. Mechatronics, 39:1-11.

Baugher, T.A. Anatomy and Taxonomy, in Baugher, T.A. \& Singha, S (eds.). 2003. Concise Encyclopaedia of Temperate Tree Fruit. New York: Food Products Press, 3-7.

Coetzee, C.J. \& Lombard, S.G. 2013. The Destemming of Grapes: Experiments and Discrete Element Modelling, Biosystems Engineering, 114(3):232-248.

Coetzee, C.J. 2017. Review: Calibration of the Discrete Element Method, Powder Technology, 310:104-142.

Cundall, P.A. \& Strack, O.D.L. 1979. Discrete Numerical Model for Granular Assemblies. Geotechnique, 29:47-65.

Diels, E., Odenthal, T., Keresztes., J., Vanmaercke., S., Verboven., P., Nicolaï., B., Saeys, W., Ramon, H. \& Smeets, B. 2016. Development of a ViscoElastoplastic Contact Force Model and its Parameter Determination for Apples. Postharvest Biology and Technology, 120:157-166.

Dintwa, E. 2006. Development of Accurate Contact Force Models for use with Discrete Element Method (DEM) Modelling of Bulk Fruit Handling Processes. PhD Thesis. Leuven: Katholieke Universiteit Leuven. 
REFERENCES

Dintwa, E., Van Zeebroeck, M., Ramon, H. \& Tijskens, E. 2008. Finite Element Analysis of the Dynamic Collision of Apple Fruit. Postharvest Biology and Technology, 49:260-276.

Dintwa, E., Van Zeebroeck, M., Tijskens, E. \& Ramon, H. 2005. Determination of Parameters of a Tangential Contact Force Model for Viscoelastic Spheroids (Fruits) using a Rheometer Device. Biosystems Engineering, 91(3):321-327.

Fadiji, T., Coetzee, C.J., Chen, L., Chukwu, O. \& Opara, U.L. 2016. Susceptibility of Apples to Bruising inside Ventilated Corrugated Paperboard Packages during Simulated Transport Damage. Postharvest Biology and Technology, 118:111119.

Fadiji, T., Coetzee, C.J., Pathare, P. \& Opara, U.L. 2016. Susceptibility to Impact Damage of Apples Inside Ventilated Corrugated Paperboard Packages: Effects of Package Design. Postharvest Biology and Technology, 111:286-296.

Faik, S. \& Witteman, H. 2000. Modelling of Impact Dynamics: A Literature Survey. Paper presented at the 2000 International ADAMS User Conference.

Favier, J.F., Abbaspour-Fard, M.H. Kremmer, M. \& Raji, A.O. 1999. Shape Representation of Axi-Symmetrical, Non-Spherical Particles in Discrete Element Simulation using Multi-Element Model Particles. Engineering Computations, 16(4): $67-480$.

Fraige, F.Y., Langston, P.A., Matchett, A.J. \& Dodds, J. 2008. Vibration Induced Flow in Hoppers: DEM 2D Polygon Model. Particuology, 6:455-466.

Golding, J. \& Jobling, J. Appels, in Rees, D., Farrell, G. \& Orchard, J (eds.). 2012. Crop Post-Harvest: Science and Technology - Perishables. Oxford, UK Malden, MA: Blackwell Science. 88-103.

Gonzalez, K.M. 2009. Bruising Profile of Fresh "Golden Delicious" Apples. PhD Thesis. Pullman, Washington: Washington State University. 
Hogue, C. \& Newland, D. 1994. Efficient Computer Simulation of Moving Granular Particles. Powder Technology, 78:51-66.

Höhner, D., Wirtz, S., Kruggel-Emden, H. \& Scherer, V. 2011. Comparison of the Multisphere and Polyhedral Approach to Simulate Non-Spherical Particles within the Discrete Element Method: Influence on Temporal Force Evolution for Multiple Contacts. Powder Technology, 208(3):643-656.

Höhner, D., Wirtz, S. \& Scherer, V. 2013. A study on the Influence of Particle Shape and Shape Approximation on Particle Mechanics in a Rotating Drum using the Discrete Element Method. Powder Technology, 253:256-265.

Holt, J. E. \& Schoorl, D. 1977. Bruising and Energy Dissipation in Apples. Journal of Textures Studies, 7:421-432.

Holt, J. E. \& Schoorl, D. 1983. Fracture in Potatoes and Apples. Journal of Materials Science, 18:2017-2028.

Horabik, J., Beczek, M., Mazur, R., Parafiniuk, P., Ryżak, M. \& Molenda, M. 2017. Determination of the Restitution Coefficient of Seeds and Coefficients of Visco-Elastic Hertz Contact Models for DEM Simulations. Biosystems Engineering, 161:106-119.

Inman, D.J. 2014. Engineering Vibration. Boston: Pearson Education.

Khodabakhshian, R. \& Emadi, B. 2011. Determination of the Modulus of Elasticity in Agricultural Seeds on the Basis of Elasticity Theory. Middle-East Journal of Scientific Research, 7(3):367-373.

Kruggel-Emden, H. Rickelt, S. Wirtz, S. \& Scherer, V. 2008. A Study on the Validity of the Multi-Sphere Discrete Element Method. Powder Technology, 188(2):153-165.

Kuang, M. 1998. Evaluation of Alternative Methods of Bruise Measurements in Apple Fruit. Masters Thesis. Palmerson North, New Zealand: Massey University. 
Kuwabara, G. \& Kono, K. 1987. Restitution Coefficient in a Collision between Two Spheres. Japanese Journal of Applied Physics, 26(8):1230-1233.

Lankarani, H.M. \& Nikravesh, P.E. 1990. A Contact Force Model with Hysteresis Damping for Impact Analysis of Multibody Systems. Journal of Mechanical Design, 112:369-376.

Lewis, R. Yoxall, A. Marshall, M.B. \& Canty, L.A. 2008. Characterising Pressure and Bruising in Apple Fruit. Wear, 264:37-46.

Lu, G., Third, J.R. \& Müller, C.R. 2012. Critical Assessment of Two Approaches for Evaluating Contacts between Super-Quadric Shaped Particles in DEM Simulations. Chemical Engineering Science, 78:226-235.

MATLAB Version 8.5.0, 2015, Computer Software, The MathWorks Inc., Natick, Massachusetts.

Mellmann, J. 2001. The Transverse Motion of Solids in Rotating Cylinders Forms of Motion and Transition Behaviour. Powder Technology, 118(3): 251270.

Mindlin, R. D. \& Deresiewicz, H. 1953. Elastic Spheres in Contact under Varying Oblique Forces. Journal of Applied Mechanics, 20:327-344.

Mitsuhashi-Gonzalez, K., Pitts, M.J., Fellman, J. K., Curry, E.A. \& Clary, C.D. 2010. Bruising Profile of Fresh Apples Associated with Tissue Type and Structure. Applied Engineering in Agriculture, 26(3):509-517.

Mohsenin, N.N. 1986. Physical Properties of Plant and Animal Materials. New York: Gordon and Breach Science Publishers.

Morrow, C.T. \& Mohsenin, N.N. 1966. Consideration of Selected Agricultural Products as Viscoelastic Materials, Pennsylvania Agricultural Experiment Station, 686-698. 
REFERENCES

Opara, U.L., Al-Ghafri, A., Agzoun, H., Al-Issai, J. \& Al-Jabri, F. 2007. Design and Development of a New Device for measuring Susceptibility to Impact Damage of Fresh Produce. New Zealand Journal of Crop and Horticultural Science, 35:245251.

Opara, U. L. \& Pathare, P. B. 2014. Bruise Damage Measurement and Analysis of Fresh Horticultural Produce - A Review. Postharvest Biology and Technology, 91:9-24.

Pang, W., Studman, C.J. \& Ward, G.T. 1992. Bruising Damage in Apple-to-Apple Impact, Journal of Agricultural Engineering Research, 52:229-240.

PFC ${ }^{3 D}$ Version 3.0, 2003, Computer Software. ITASCA Consulting Group, Inc., Minneapolis, Minnesota.

PFC ${ }^{3 D}$ Version 5.0, 2016, Computer Software. ITASCA Consulting Group, Inc., Minneapolis, Minnesota.

Sarracino, R.S., McBride, A.T. \& Powell, M.S. 2004. Using Particle Flow Code to Investigate Energy Dissipation in a Rotary Grinding Mill, in Shimizu, Y., Hart, R. \& Cundall. (eds.). Numerical Modelling in Micromechanics via Particle Methods. London: Taylor \& Francis Group. 111-118.

Schoorl, D. \& Holt, J. E. 1977. The Effects of Storage Time and Temperature on the Bruising of Jonathan, Delicious and Granny Smith Apples. Journal of Texture Studies, 8:409-416.

Schoorl, D. \& Holt, J. E. 1980. Bruise Resistance Measurement in Apples. Journal of Texture Studies, 11:389-394.

Taghavi, R. 2011. Automatic Clump Generation Based on Mid-Surface. Minneapolis, MN: Itasca Consulting Group, Inc.

Thornton, C. \& Ning, Z. 1997. A theoretical Model for the Stick/Bounce Behaviour of Adhesive, Elastic-Plastic Spheres. Powder Technology, 99:154-162. 
REFERENCES

Tijskens, E., Ramon, H. \& De Baerdemarker, J. 2003. Discrete Element Modelling for Process Simulation in Agriculture. Journal of Sound and Vibration, 266:493-514.

Van Zeebroeck, M. 2005. The Discrete Element Method (DEM) to Simulate Fruit Impact Damage during Transport and Handling. PhD Thesis. Leuven: Katholieke Universiteit Leuven.

Van Zeebroeck, M., Dintwa, E., Tijskens, E., Deli, V., Loodts, J., De Baerdemaeker, J. \& Ramon, H. 2004. Determining Tangential Contact Model Parameters for Viscoelastic Materials (Apples) using a Rheometer. Postharvest Biology and Technology, 33:111-125.

Van Zeebroeck, M., Tijskens, E., Dintwa, E., Kafashan, J., Loodts, J., De Baerdemaeker, J. \& Ramon, H. 2006. The Discrete Element Method (DEM) to Simulate Fruit Impact Damage during Transport and Handling: Case Study of Vibration Damage during Apple Bulk Transport. Postharvest Biology and Technology, 41:92-100.

Van Zeebroeck, M., Tijskens, E., Dintwa, E., Kafashan, J., Loodts, J., De Baerdemaeker, J. \& Ramon, H. 2006. The Discrete Element Method (DEM) to Simulate Fruit Impact Damage during Transport and Handling: Model Building and Validation of DEM to Predict Bruise Damage of Apples. Postharvest Biology and Technology, 41:85-91.

Van Zeebroeck, M., Tijskens, E., Van Liedekerke, P., Deli, V., De Baerdemaeker, J. \& Ramon, H. 2003. Determination of the Dynamical Behaviour of Biological Materials during Impact Using a Pendulum Device. Journal of Sound and Vibration, 266:465-480.

Van Zeebroeck, M., Van Linden, V., Darius, P., De Ketelaere, B., Ramon, H. \& Tijskens, E. 2007. The Effect of Fruit Factors on the Bruise Susceptibility of Apples. Postharvest Biology and Technology, 46:10-19. 
REFERENCES

Van Zeebroeck, M., Van Linden, V., Ramon, H., De Baerdemaeker, J., Nicolaï. B.M. \& Tijskens, E. 2007. Impact Damage of Apples during Transport and Handling. Postharvest Biology and Technology, 45:157-167.

Vigneault, C. Thompson, J, Wu, S. Hui, K.P.C. \& LeBlanc, D. I. 2009.

Transportation of Fresh Horticultural Produce, Benkeblia, N. (ed.). Postharvest Technologies for Horticultural Crops. Trivandrum, Kerala, India: Research Signpost. 2:1-24.

Wachs, A., Girolami, L., Vinay, G. \& Ferrer, G. 2012. Grains3D, a Flexible DEM Approach for Particles of Arbitrary Convex Shape - Part I: Numerical Model and Validations. Powder Technology. 224:374-389.

Yang, S., Sun, Y., Zhang, L. \& Chew, J.W. 2017. Segregation Dynamics of a Binary-Size Mixture in a Three-Dimensional Rotating Drum. Chemical Engineering Science, 172:652-666.

Zhu, H.P. \& Yu, A.B. 2006. A Theoretical Analysis of the Force Models in Discrete Element Method. Powder Technology, 161:122-129. 


\section{APPENDIX A: CONTACT MODEL PARAMETER ACQUISITION}

The experimental work of Chapter 3 and Chapter 4 required the use of a force transducer and accelerometer. The calibration data for the measuring instruments is accordingly presented. Supplementary information detailing the parameter acquisition of Chapter 3.3 is also presented. Additionally, the method for determining the radius of curvature is described.

\section{A.1. Calibration of Instrumentation}

To obtain accurate results when performing the pendulum experiments, a comprehensive calibration of the force transducer was completed. A static calibration was done where the magnitudes of the known input weights were measured against the output voltage. Weights corresponding to the loads that the force transducer would experience were used. The force calibration curve is shown in Figure A.1 with the correlation equation presented.

The load cell was manufactured by the Vishay Precision Group, Inc. (VPG). A revere model 363 multi-purpose nickel-plated S-Type load cell was used. The load cell featured a $50 \mathrm{~kg}$ Capacity and a sensitivity of $0.73315 \mathrm{mV} / \mathrm{V}$.

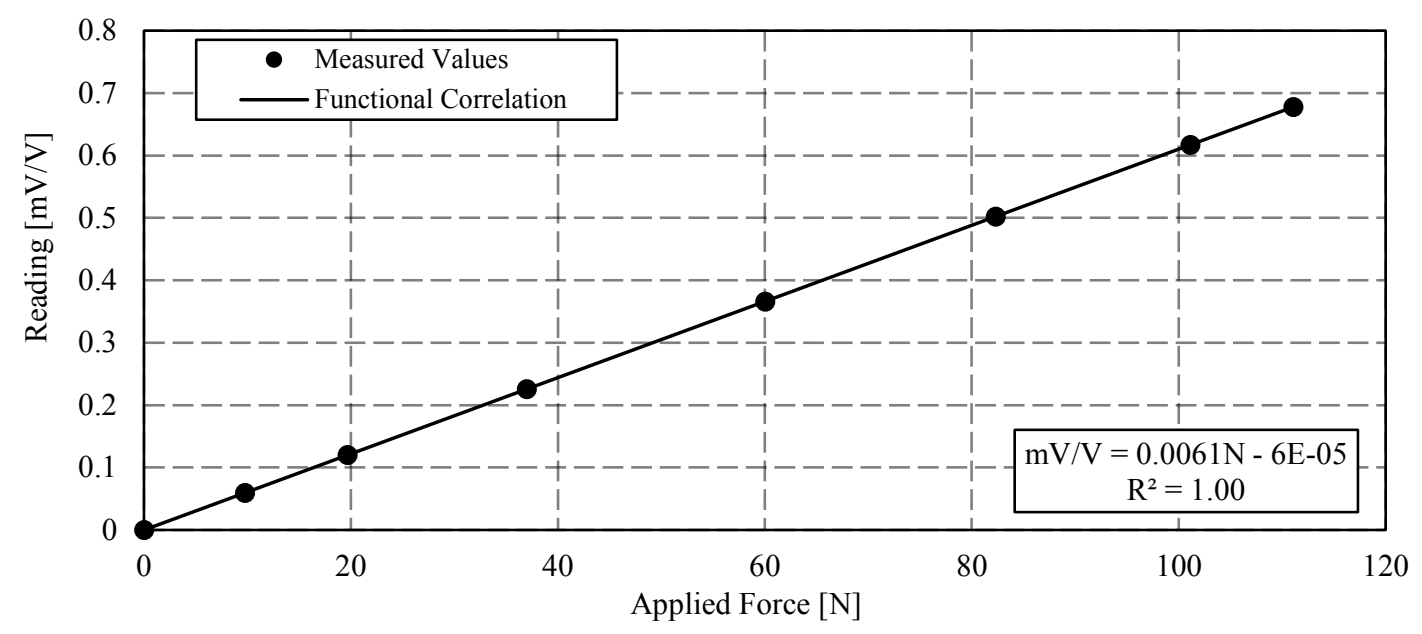

Figure A.1: Force Calibration Curve

The calibration data card for the accelerometer used for the attainment of the deformation and deformation rate is given in Table A.1. 
APPENDIX A: CONTACT MODEL PARAMETER ACQUISITION

Table A.1: Accelerometer calibration information

\begin{tabular}{l|l}
\hline \multicolumn{2}{c}{ SHEAR ACCELEROMETER } \\
\hline Model: & $\# 333 \mathrm{~B} 32$ \\
Serial: & $\# 18857$ \\
Sensitivity: & $98.9 \mathrm{mv} / \mathrm{g}$ \\
Range: & $0.5-3000 \mathrm{~Hz}$ \\
Bias Level: & $10.9 \mathrm{~V}$ \\
\hline
\end{tabular}

PCB PIEZOTRONICS: Vibration Division, 3425 Walden Ave. Depew, NY 14043

The verification of the displacement-time curves obtained with the use of an accelerometer is discussed in Section 3.2.2. Tests were performed where impacts from the spherical impactor were simultaneously recorded with the accelerometer and a high-speed camera. The images produced by the high-speed camera were subsequently imported into MATLAB and a contour plot produced which could identify a white dot marked on the spherical impactor as shown in Figure A.2. This dot was subsequently tracked for every time frame and together with the pixel scale $(0.147 \mathrm{~mm} /$ pixel $)$, force-time curves were produced from the high-speed camera.

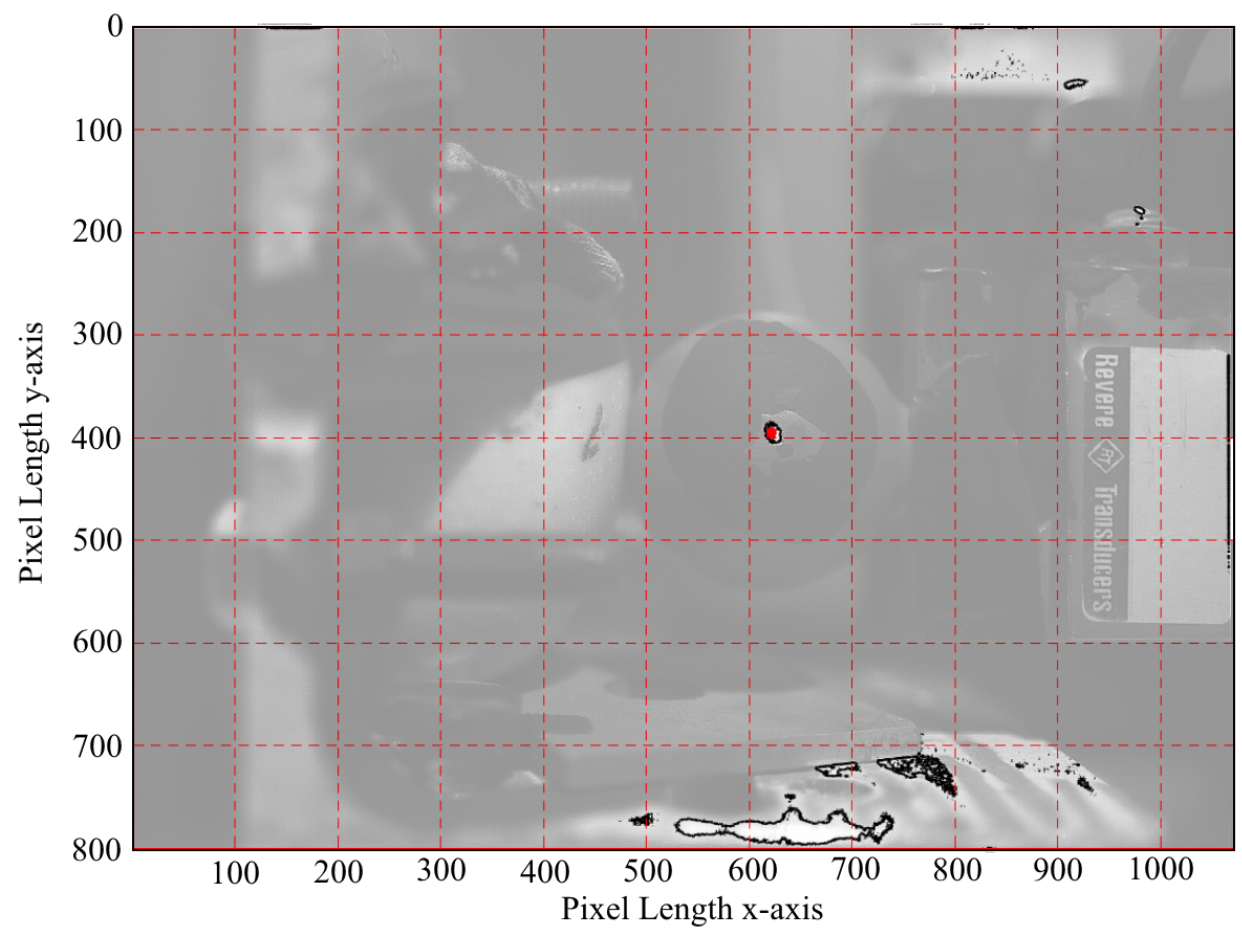

Figure A.2: Contour plot of grayscale image of impact test recorded using highspeed camera 


\section{APPENDIX A: CONTACT MODEL PARAMETER ACQUISITION}

As discussed in Chapter 3.2.2 an Impulse Force Test Hammer (PCB Piezotronics, MTS Systems Corporation. PCB MD 086C01) was used to confirm the accuracy of the load cells response to impact. The load cell was accordingly excited with the use of the impact hammer (at the sampling frequencies detailed in Chapter 3.2.2) and the response of the load cell and impulse hammer compared as shown in Figure A.3. Excellent agreement between the load cell and the impulse hammer was achieved.

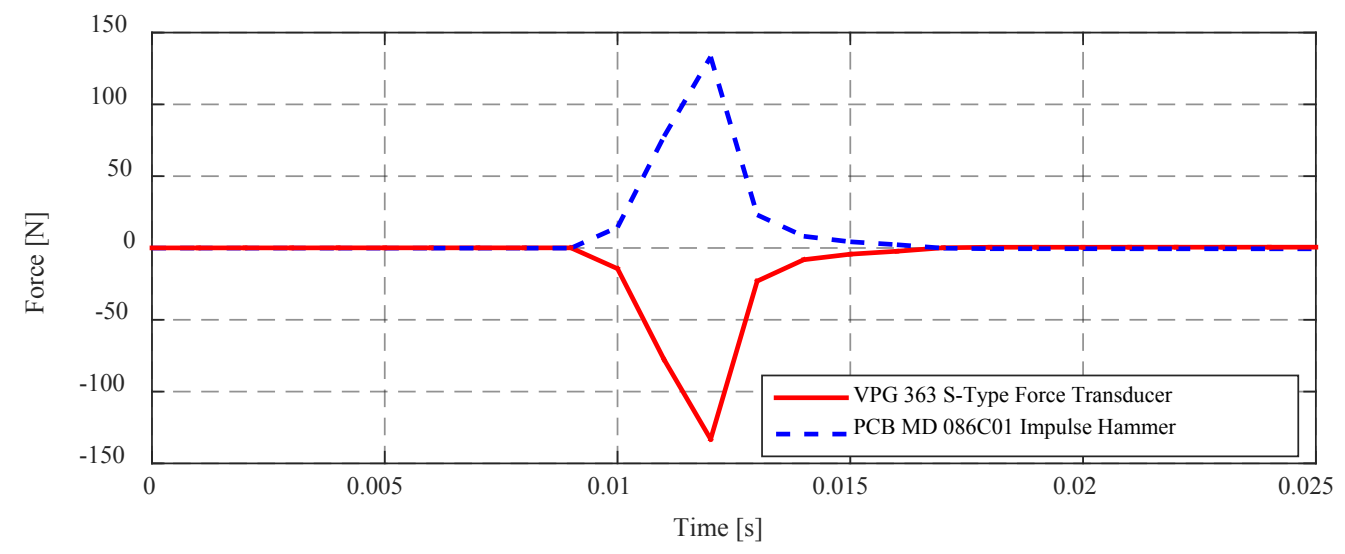

Figure A.3: Comparison of the impact response between the load cell and impact hammer

\section{A.2. Error Minimisation}

This section presents supplementary information for the error minimisation algorithm discussed in Chapter 3.3. An overview of the algorithm's procedure and order of actions is graphically represented with the use of a flowchart in Figure A.4.

The algorithm made use of each impact test's measured data. The algorithm started by finding the impact interval within the measured data. Hereafter, a search region for the normal elastic constant and normal damping constant was set. An equal step size for the elastic constant and damping constant was determined and together with the deformation and deformation rate vectors, a force vector was calculated with the use of equation 3.3.

Next, the sum of the residual errors between the calculated and measured force vectors was determined. Subsequently, the elastic constant and damping constant 


\section{APPENDIX A: CONTACT MODEL PARAMETER ACQUISITION}

corresponding to the minimum sum of residual errors was found. Lastly, the correlation coefficient was determined for the optimal constant pairing. Surface plots showing the effect of the normal elastic constants and normal damping constants on the residual errors are given in Figure A.5 through Figure A.7.

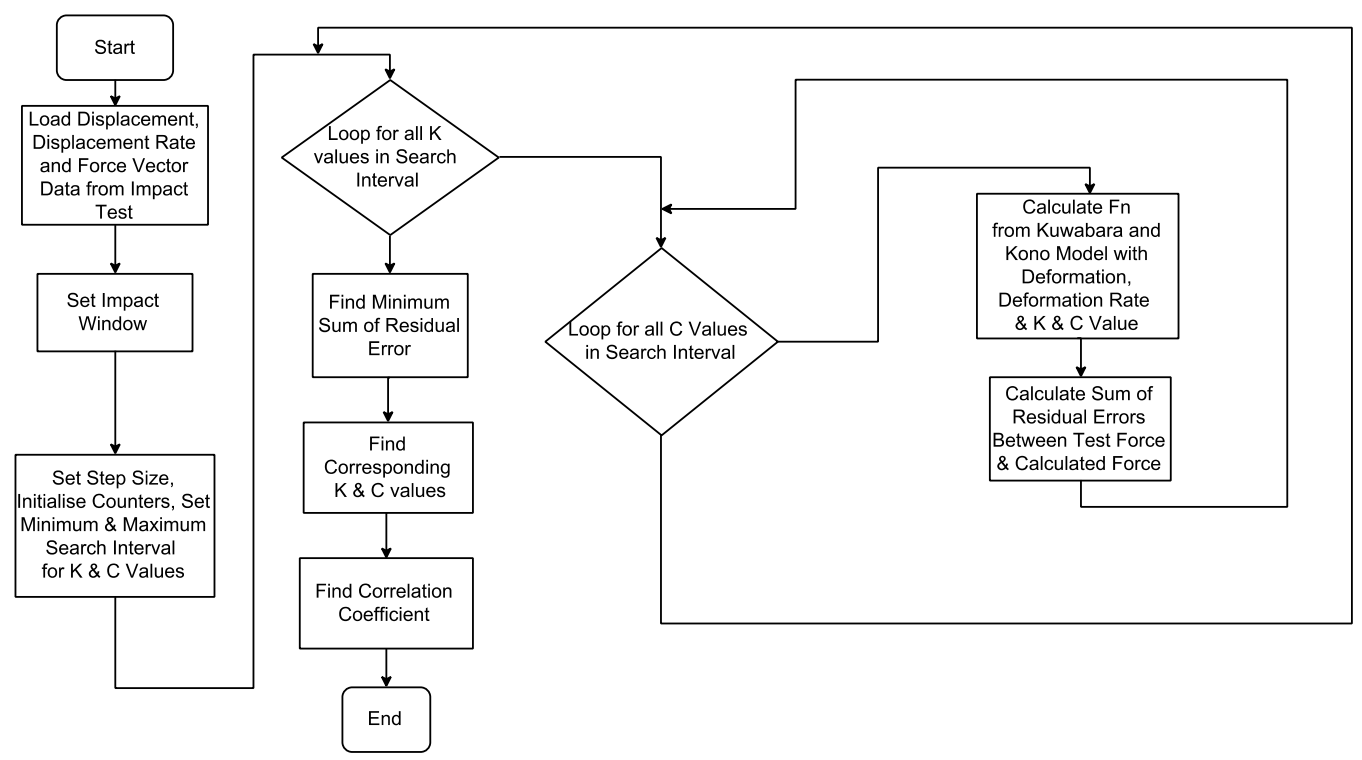

Figure A.4: Basic outline of the error minimisation algorithm

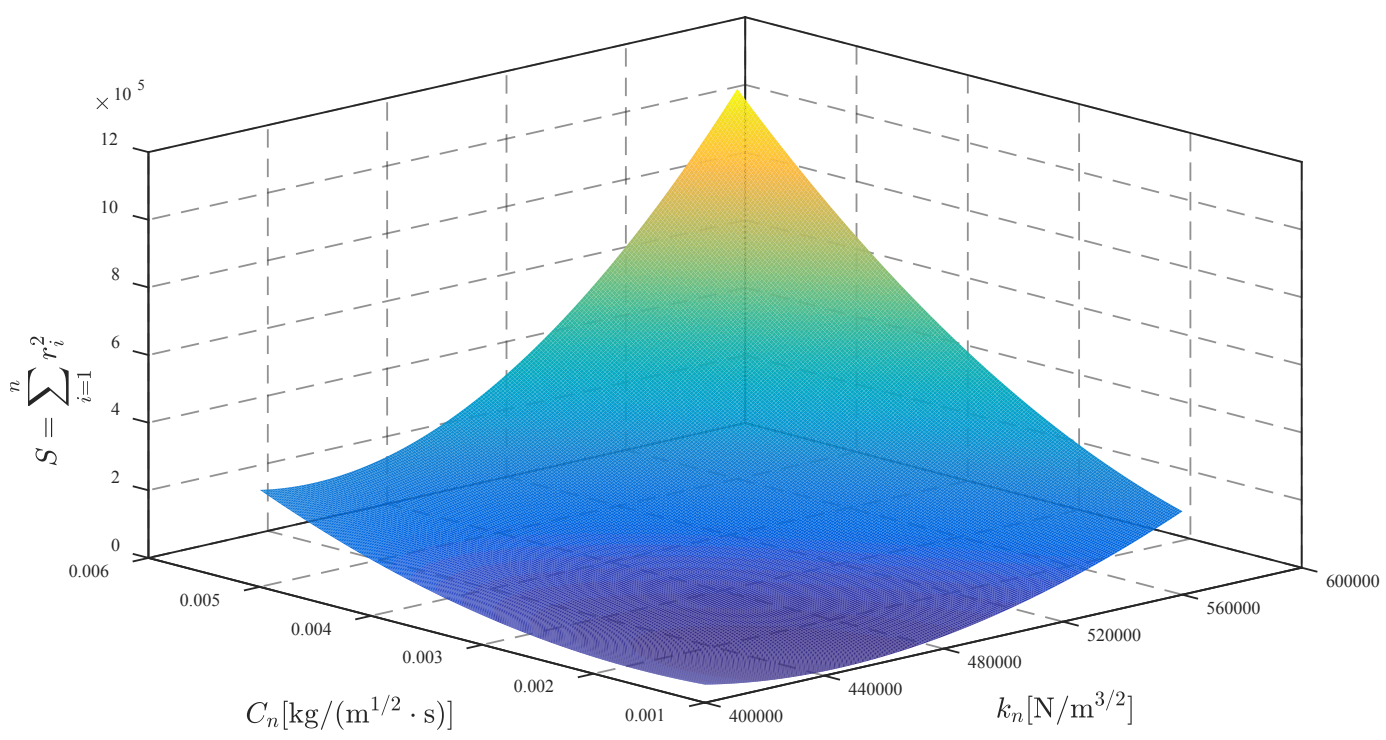

Figure A.5: Combination effect of normal damping constant and normal elastic constant on the sum of residual errors 
APPENDIX A: CONTACT MODEL PARAMETER ACQUISITION

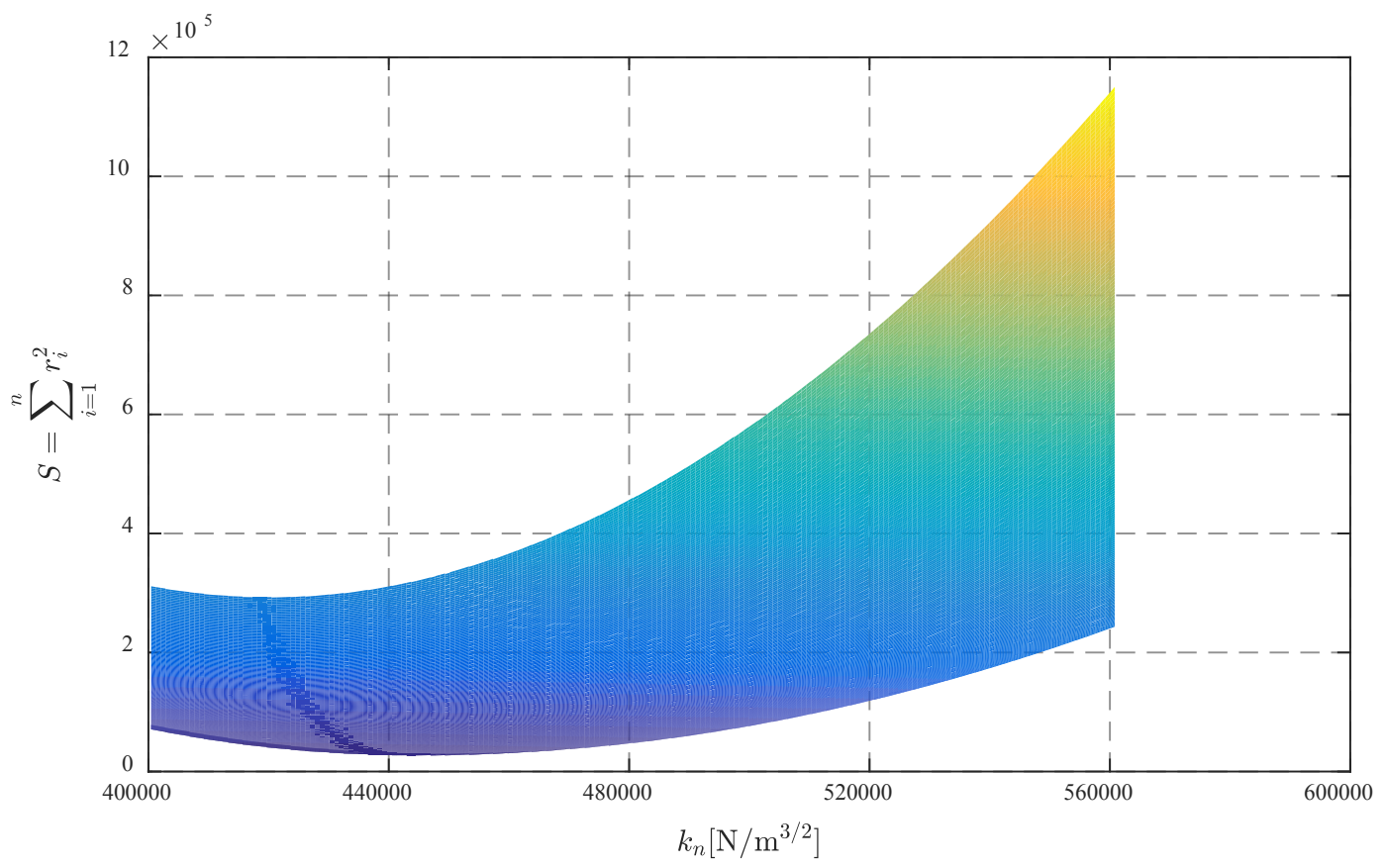

Figure A.6: Effect of normal elastic constant on the sum of residual errors

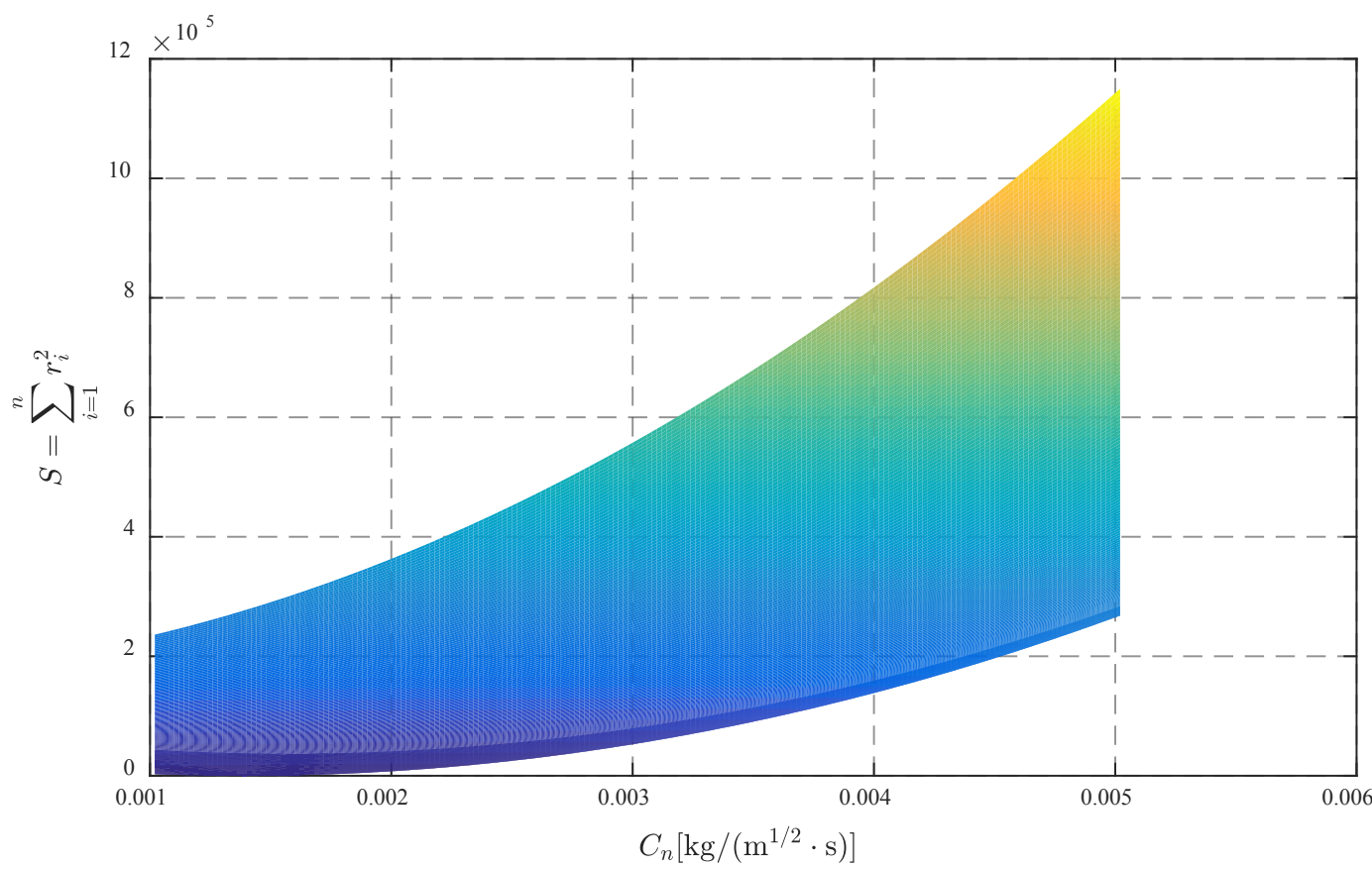

Figure A.7: Effect of normal damping constant on the sum of residual errors 


\section{APPENDIX A: CONTACT MODEL PARAMETER ACQUISITION}

\section{A.3. Radius of Curvature Measurement}

When applying the Discrete Element Method (DEM) to materials in the agricultural sector it is important to have an accurate and accepted way to obtain properties related to their shape and size. Materials that do not occur naturally, typically go through some kind of manufacturing process and therefore result in very regular well-defined shapes. The ever-expanding application of DEM into the agricultural sector will typically mean biological materials will have to be investigated. The complete theoretical specification of the form of irregularly shaped agricultural products would require an infinite number of measurements. (Mohsenin, 1986:81).

Many engineering parameters require knowledge of the dimensional characteristics of the objects under investigation. Fortunately, on a practical level, a shape can be defined by measuring several mutual perpendicular axes with the measurements increasing with particle irregularity (Mohsenin, 1986:81). For the purposes of a DEM application, the object's dimensions will typically be mandatory for investigations into the elastic and damping parameters involved in the contact model. This investigation required accurate knowledge of the effective radius of curvature of the apples at the contact location. The effective radius of curvature has a significant effect on the elastic constant (shown in equation 2.3). Accordingly, the radius of curvature was obtained using the method described by Mohsenin (1986:833) and is given by equation A. 1 with an illustration of the measurements given in Figure A.8. Each apple half tested in Chapter 3 was accordingly measured in this way with the use of a Vernier calliper. Subsequently, the radius of curvature of the apple halves were calculated using equation A.1.

$$
R^{\prime}=\frac{(A C)^{2}}{8(B D)}+\frac{B D}{2}
$$

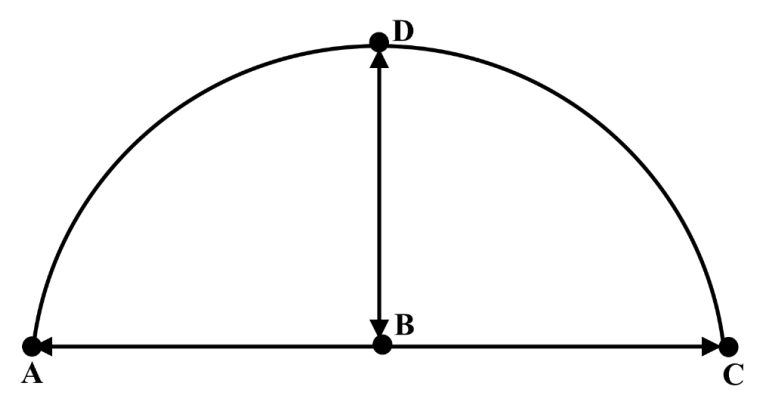

Figure A.8: Radius of curvature measurement 


\section{APPENDIX B: BRUISE DAMAGE CORRELATIONS}

\section{APPENDIX B: BRUISE DAMAGE CORRELATIONS}

The derivation of the bruise damage correlations for the determination of the bruise prediction model is presented in Chapter 3 . In order to streamline the main body of the text, the bruise damage correlations accompanying those presented in Chapter 3 are presented in Appendix B. More detailed information regarding the $\mathrm{BA}$ and $\mathrm{BD}$ relations to the peak impact force and the respective impactors used is given in Figure B.1 through Figure B.4.

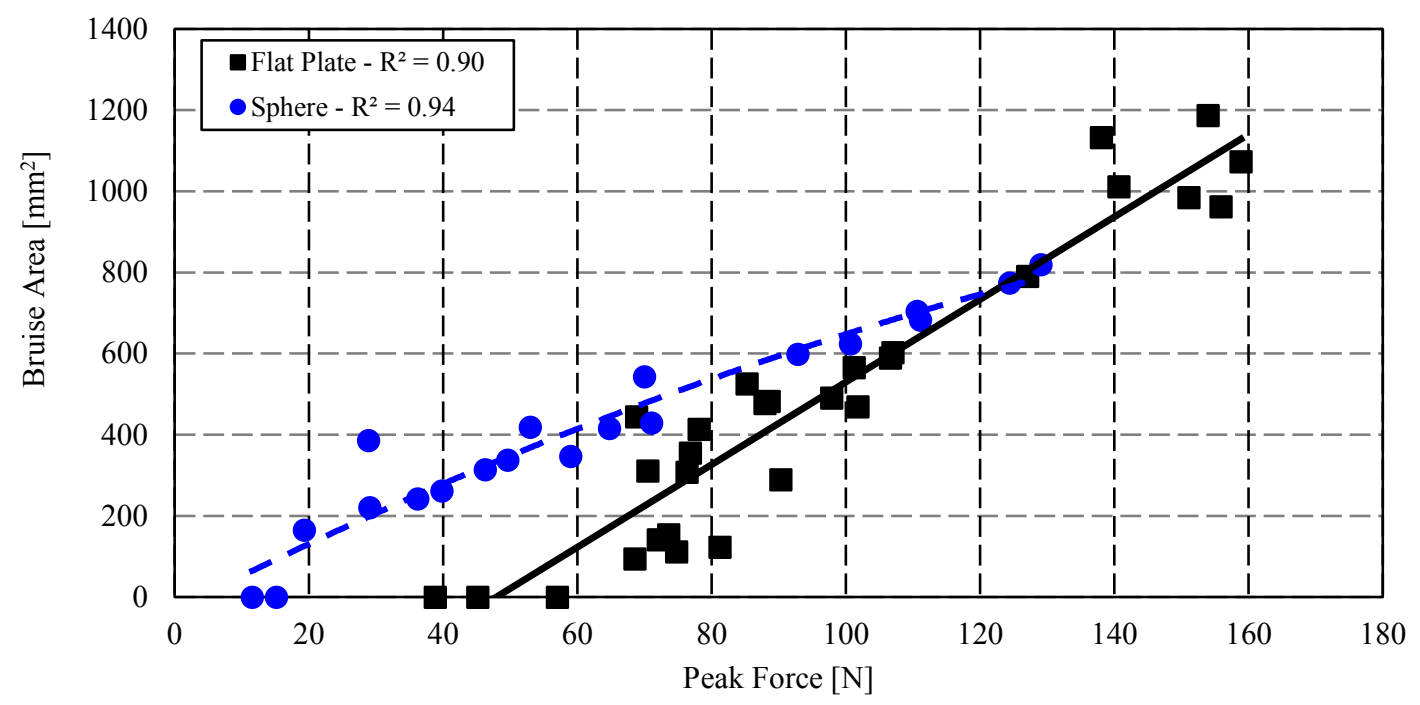

Figure B.1: Correlation between peak force and BA for spherical and flat plate impactors

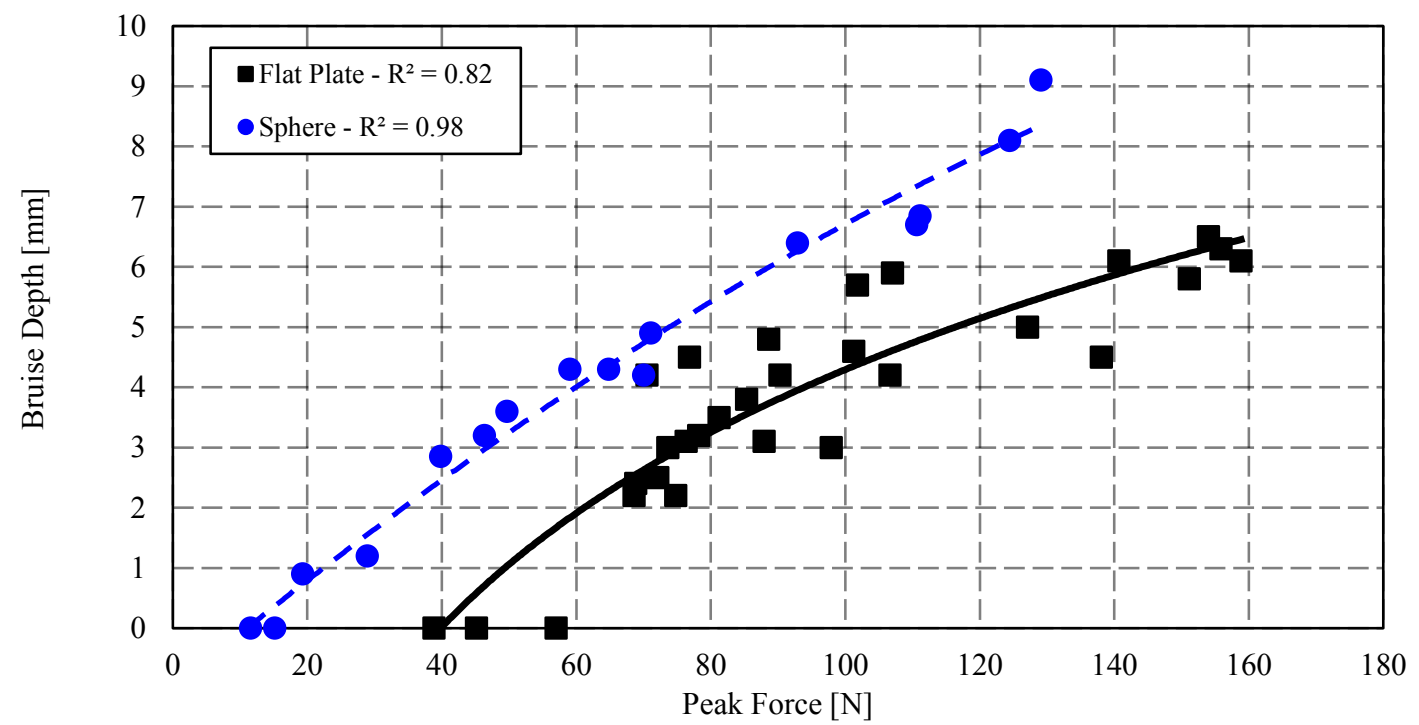

Figure B.2: Correlation between peak force and BD for spherical and flat plate impactors 
Figure B.1 and Figure B.2 supplement the data between peak force and bruise damage for Figure 4.2 and Figure B.3 and Figure B.4 supplements the data of Figure 4.3.

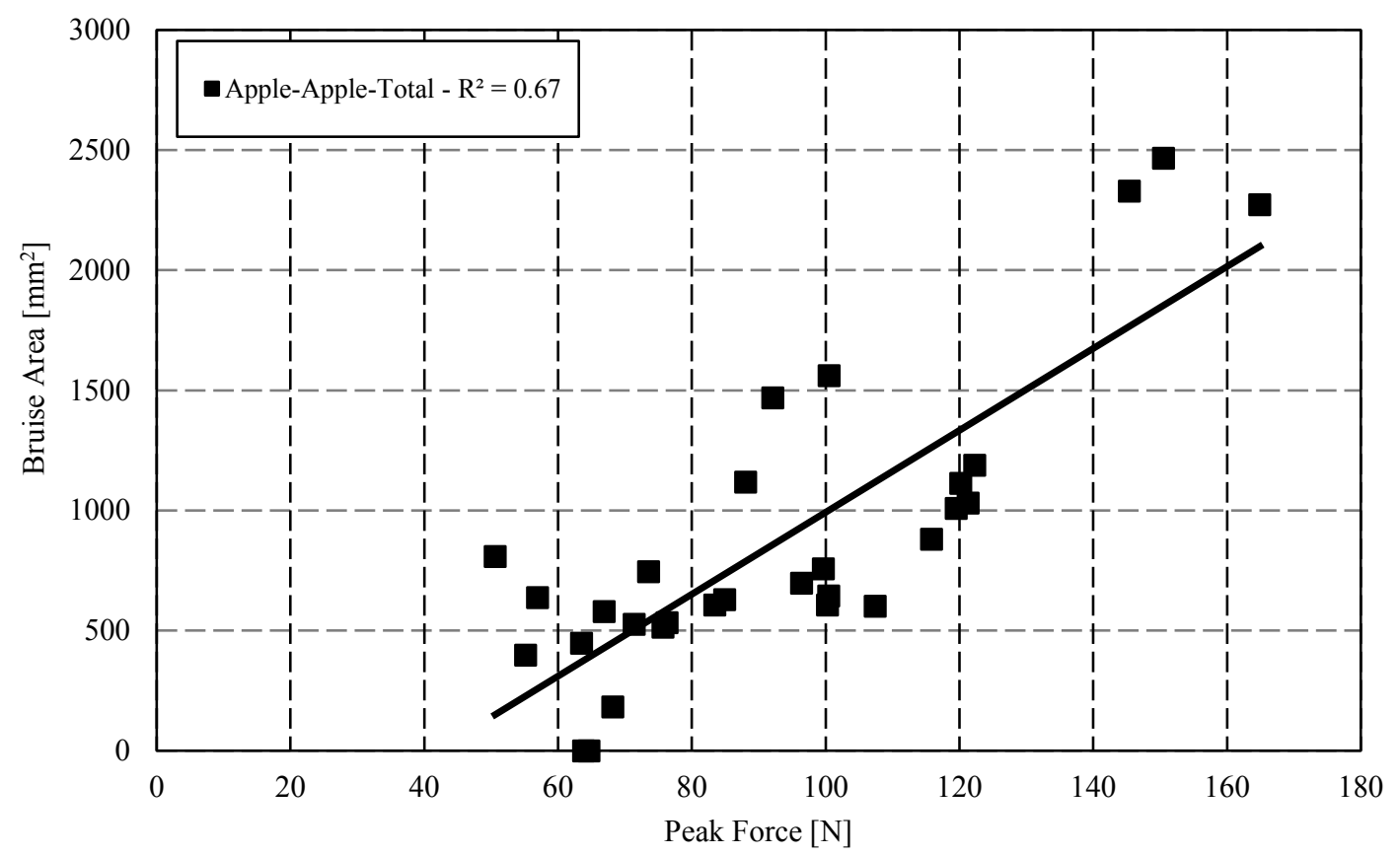

Figure B.3: Correlation between peak force and BA for apple-apple contact

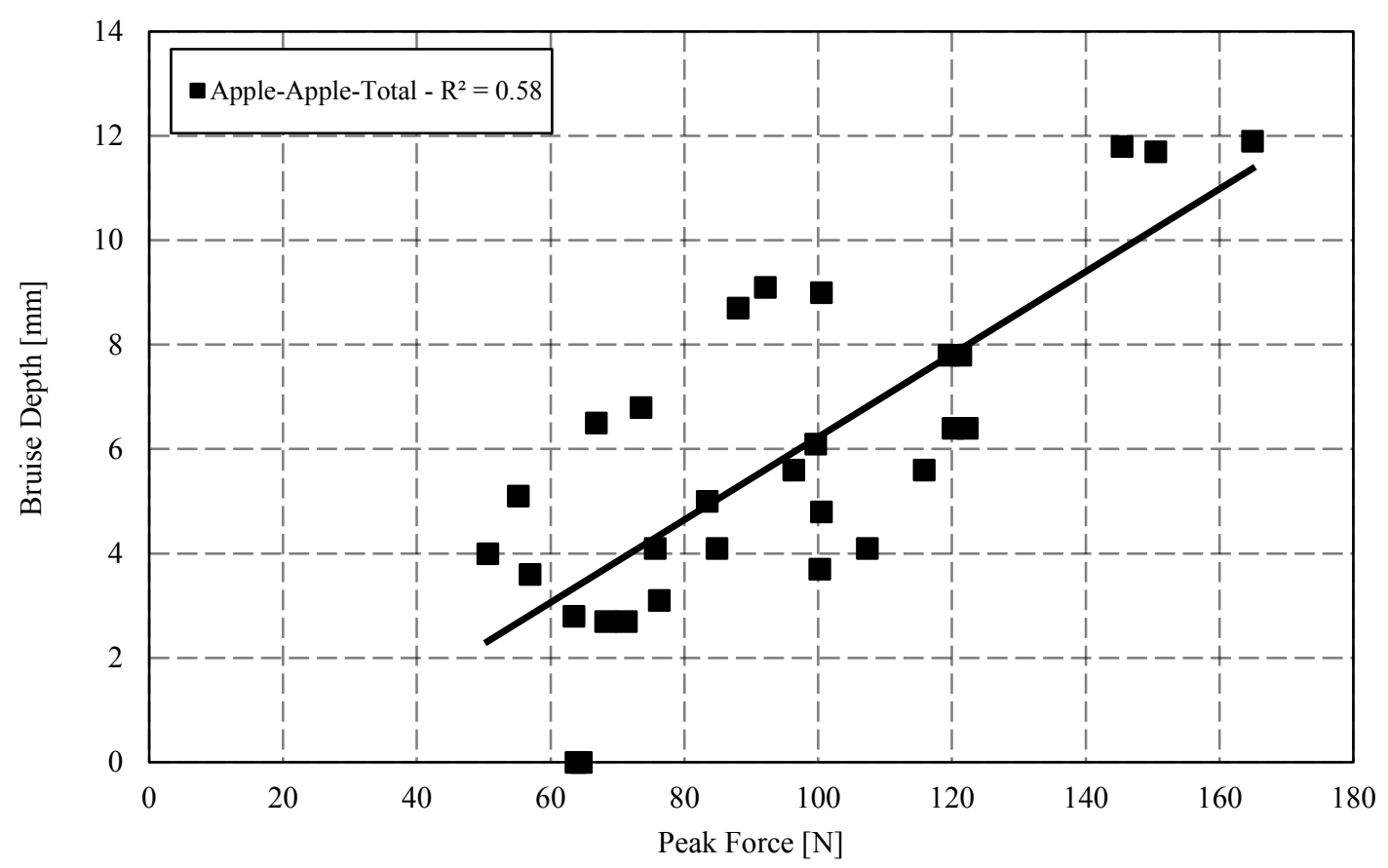

Figure B.4: Correlation between peak force and BD for apple-apple contact 
The effect of time between impacts was investigated in Chapter 4.5. The BV resulting from two impacts on the same position spaced $0-24$ hours apart is presented in Figure 4.4. The BA and BD data acquired from the same impacts of Figure 4.4 is presented in Figure B.5 and Figure B.6 respectively.

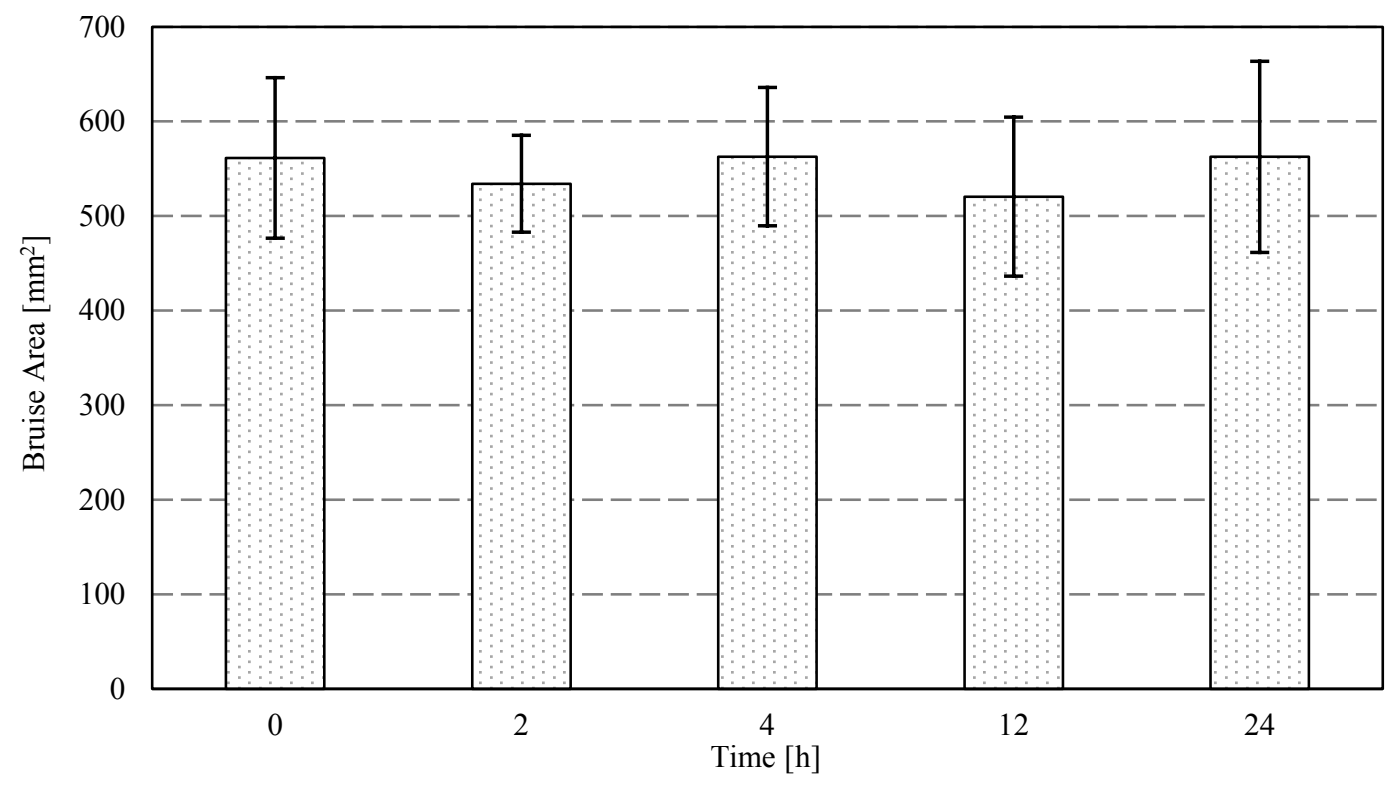

Figure B.5: Resulting BA from two impacts spaced $0-24 \mathrm{~h}$ apart. Error bars indicate $1 \mathrm{SD}$

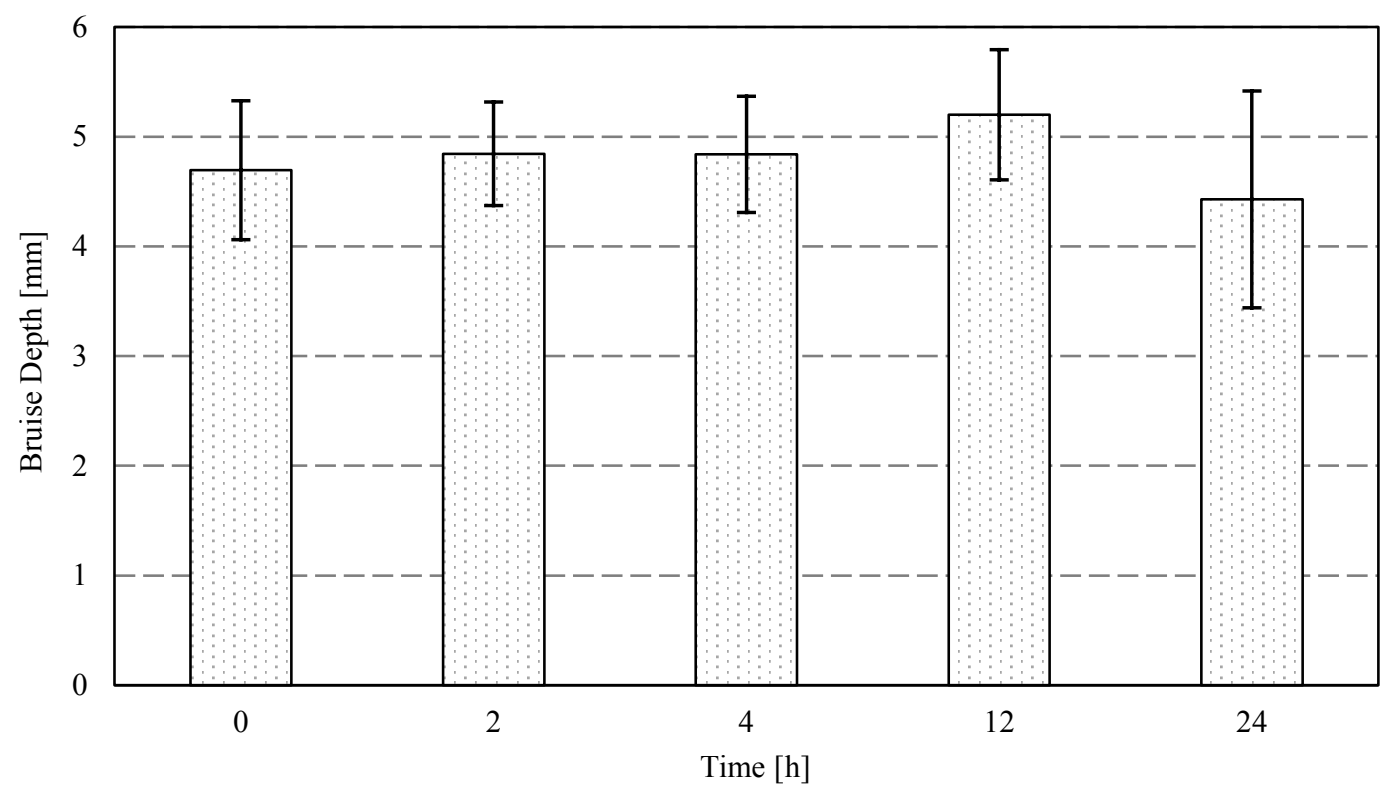

Figure B.6: Resulting BD from two impacts spaced $0-24 \mathrm{~h}$ apart. Error bars indicate $1 \mathrm{SD}$ 


\section{APPENDIX B: BRUISE DAMAGE CORRELATIONS}

The effect of successive immediate impacts of different magnitudes on the same position was also explored in Chapter 4.5. The BV's resulting from the experiments are given in Figure 4.5. Figure B.7 and Figure B.8 supplement the data set and show the resulting $B A$ and $B D$ of the same impacts as those of Figure 4.5.

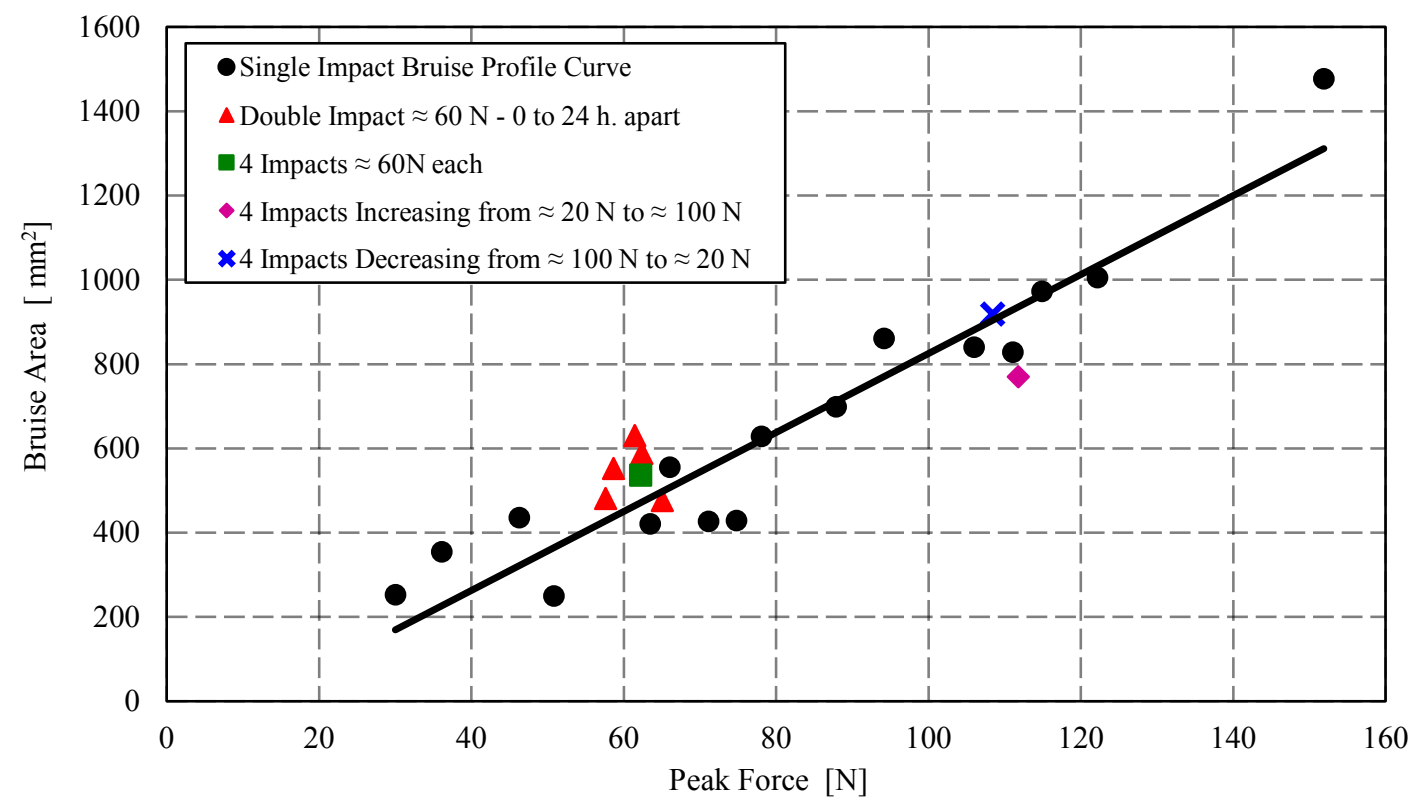

Figure B.7: Bruise area resulting from multiple impacts compared to single impact profile

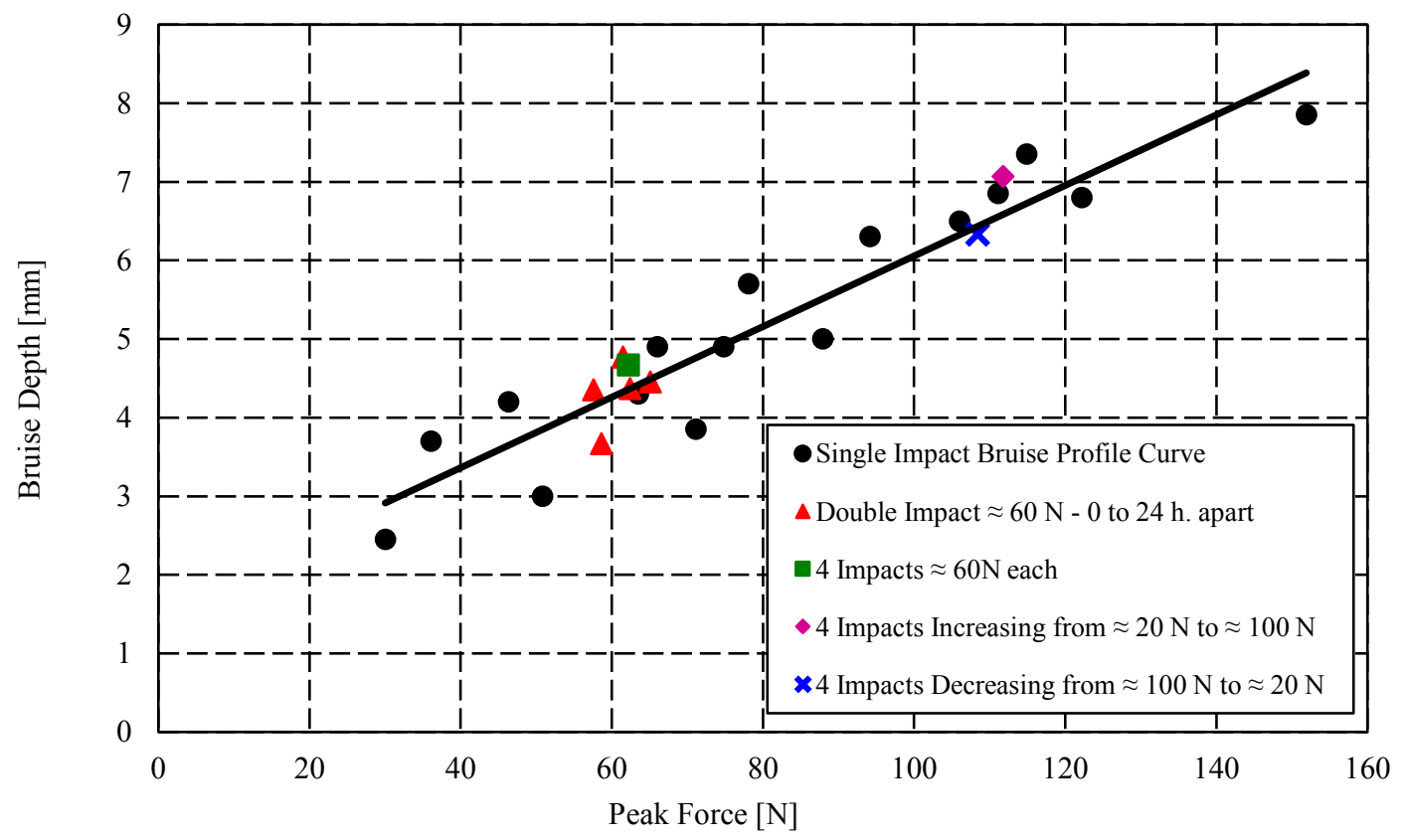

Figure B.8: Bruise depth resulting from multiple impacts compared to single impact profile 


\section{APPENDIX B: BRUISE DAMAGE CORRELATIONS}

In Chapter 5.6 the accuracy of the overlap formulation was tested. Comparisons between the BV measured experimentally and predicted numerically are given in Figure 5.10. Figure B.9 and Figure B.10 supplement the data set of Figure 5.10 and show the corresponding $\mathrm{BA}$ and $\mathrm{BD}$ respectively.

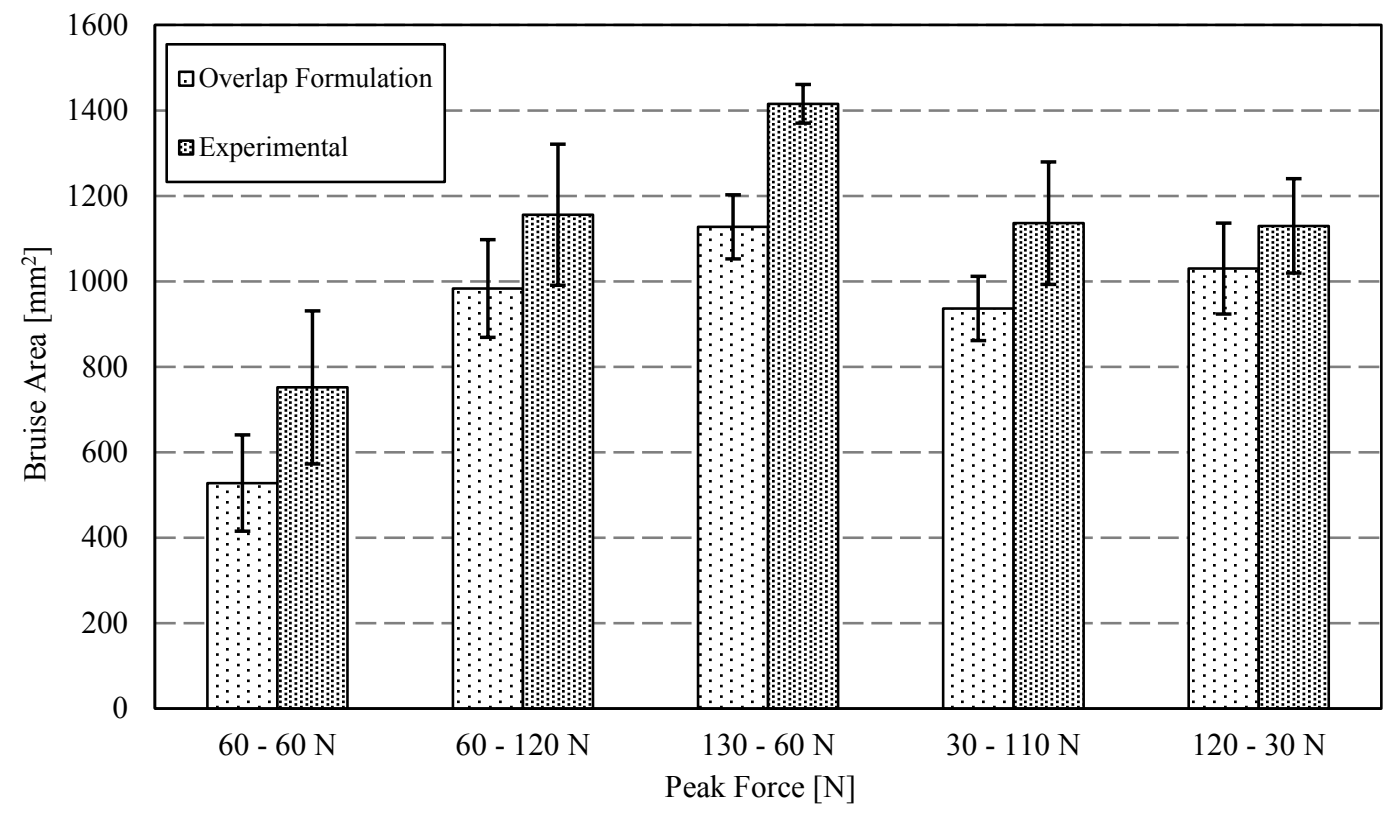

Figure B.9: Comparison between the overlap formulation and experimental overlapping bruises for the resulting BA. Error bars indicate 1 SD for 9 repetitions

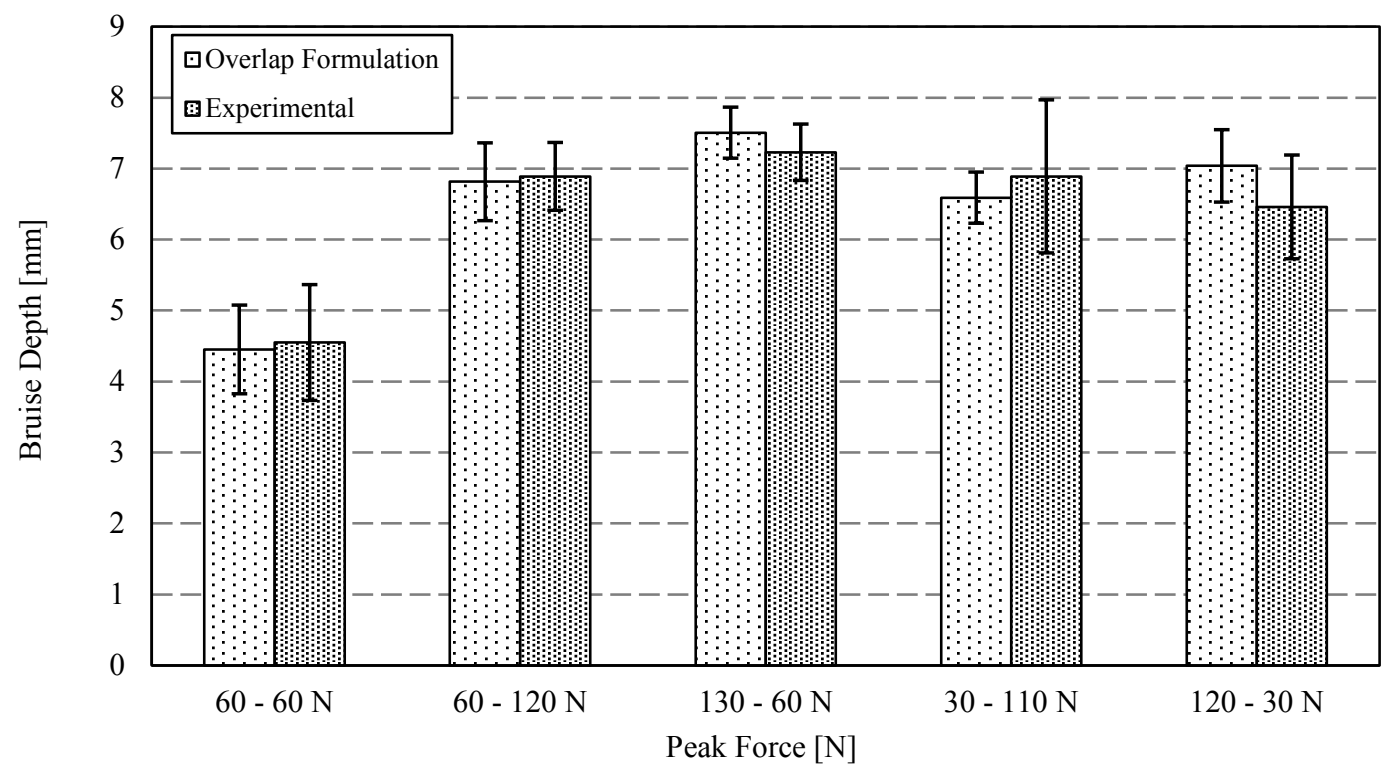

Figure B.10: Comparison between the overlap formulation and experimental overlapping bruises for the resulting BD. Error bars indicate 1 SD for 9 repetitions 


\section{APPENDIX C: IMPACT/ABSORBED ENERGY RELATIONS}

\section{APPENDIX C: IMPACTIABSORBED ENERGY RELATIONS}

Various researchers have made use of impact or absorbed energy to investigate bruising damage to apples (Holt \& Schoorl, 1977., Mohsenin, 1986., Schoorl \& Holt, 1977., Schoorl \& Holt, 1980., Opara et al., 2007. \& Pang et al., 1992). These studies made use of drop tests, falling masses, pendulums or impact rams to correlate either the impact energy or absorbed energy to bruise damage. Researchers made use of the principles of potential or kinetic energy to determine the energy with which a specimen was impacted and the energy absorbed during an impact. Relating bruise damage to energy does not lend itself to easy implementation in DEM. Additionally, contact properties specific to the contact situation are not always accounted for when relating energies to bruise damage. From Chapter 4.3 it is shown that bruise damage was strongly dependant on the geometric and material properties of the contact situation with unique bruise damage correlations found for each impactor described in Chapter 3.2.2. When correlated with impact energy it was expected that the same order of bruise damage was present regardless of the impactor used.

Accordingly, 13 test samples for each impactor were prepared as detailed in Chapter 3.4. The bruise damage was measured as before. In order to determine the impact energy, the force-deformation curves (detailed in Chapter 3.2.2) were used to calculate the work energy by numerically integrating the force-deformation graph until the point of maximum loading was reached. In order to determine the absorbed energy, the unloading path of the hysteresis curve was numerically integrated. Subsequently, the loading and unloading work energy was subtracted from one another. The numerical integration was performed in MATLAB by making use of the trapezoidal numerical integration method. The correlation between impact energy and BV is given in Figure C.1, BA is given in Figure C.2 and BD correlation is given in Figure C.3. From Figure C.1, Figure C.2 and Figure C.3 it is clear that there was no discernible difference between the impact energy and bruise damage with regards to the impactor used. The absorbed energy correlation for BV is given in Figure C.4, for BA in Figure C.5 and for BD in Figure C.6. Again no discernible difference was observed. Furthermore, the order of the BV and absorbed energy relation of Figure C. 4 is in agreement with that reported by Holt and Schoorl (1977:427) for dynamic impacts. 


\section{APPENDIX C: IMPACT/ABSORBED ENERGY RELATIONS}

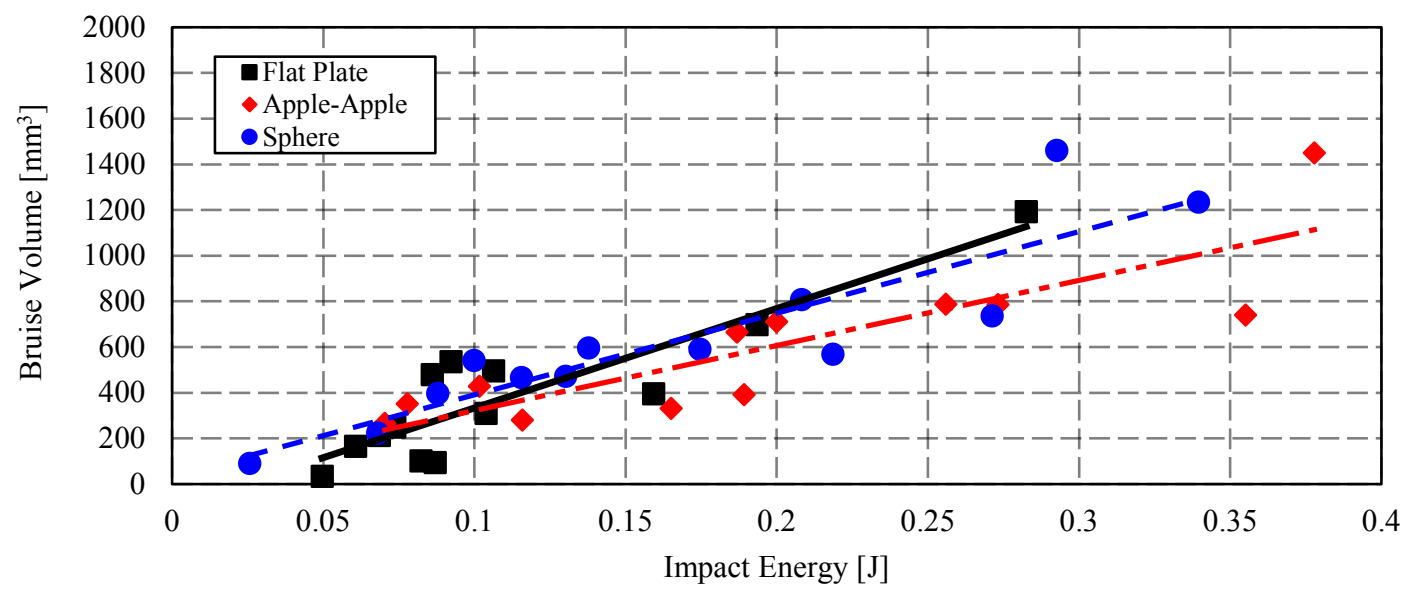

Figure C.1: Correlation between impact energy and BV for spherical, flat plate and apple impactors

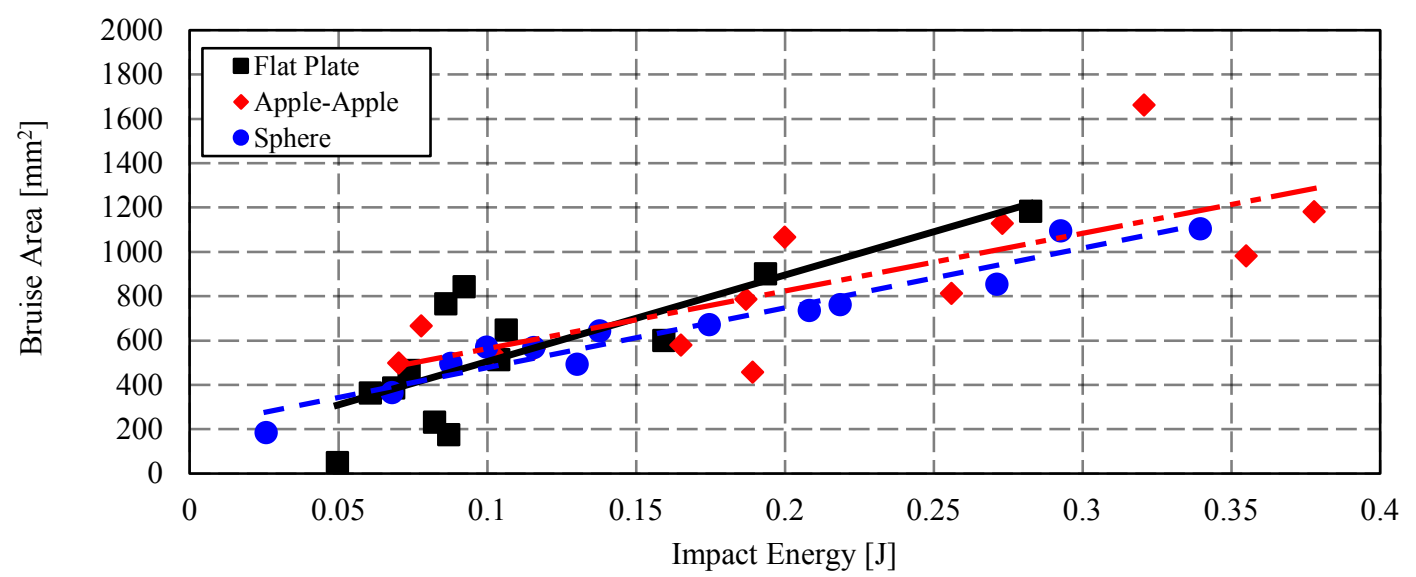

Figure C.2: Correlation between impact energy and BA for spherical, flat plate and apple impactors

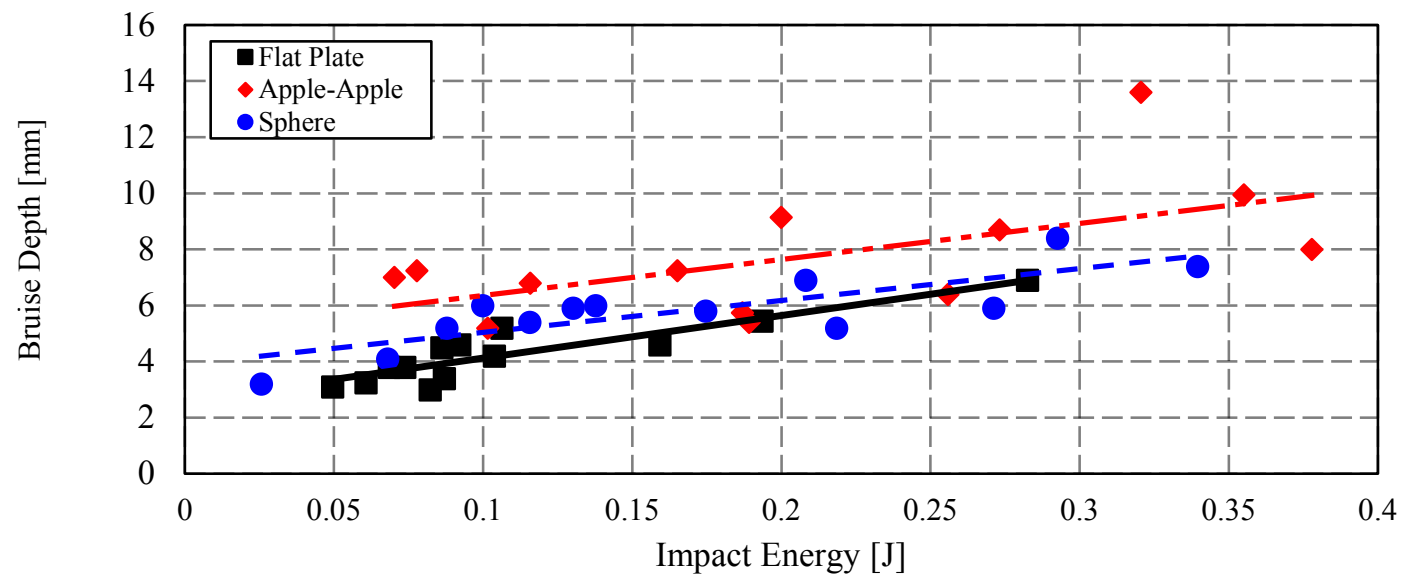

Figure C.3: Correlation between impact energy and BD for spherical, flat plate and apple impactors 
APPENDIX C: IMPACT/ABSORBED ENERGY RELATIONS

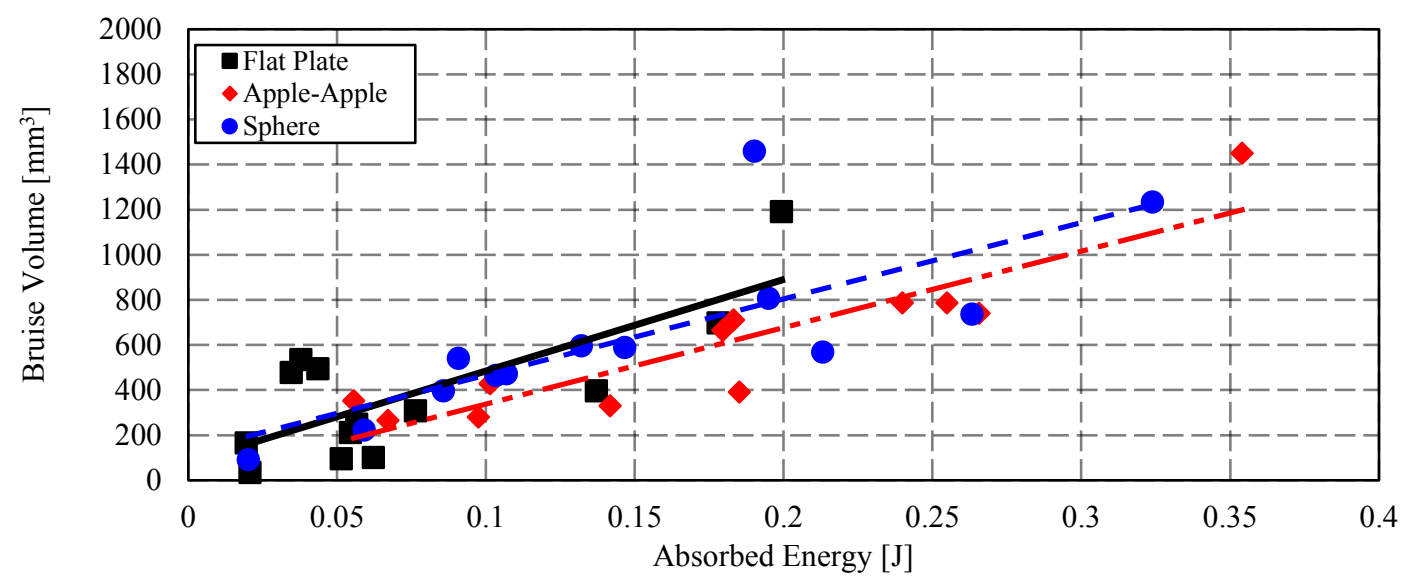

Figure C.4: Correlation between absorbed energy and BV for spherical, flat plate and apple impactors

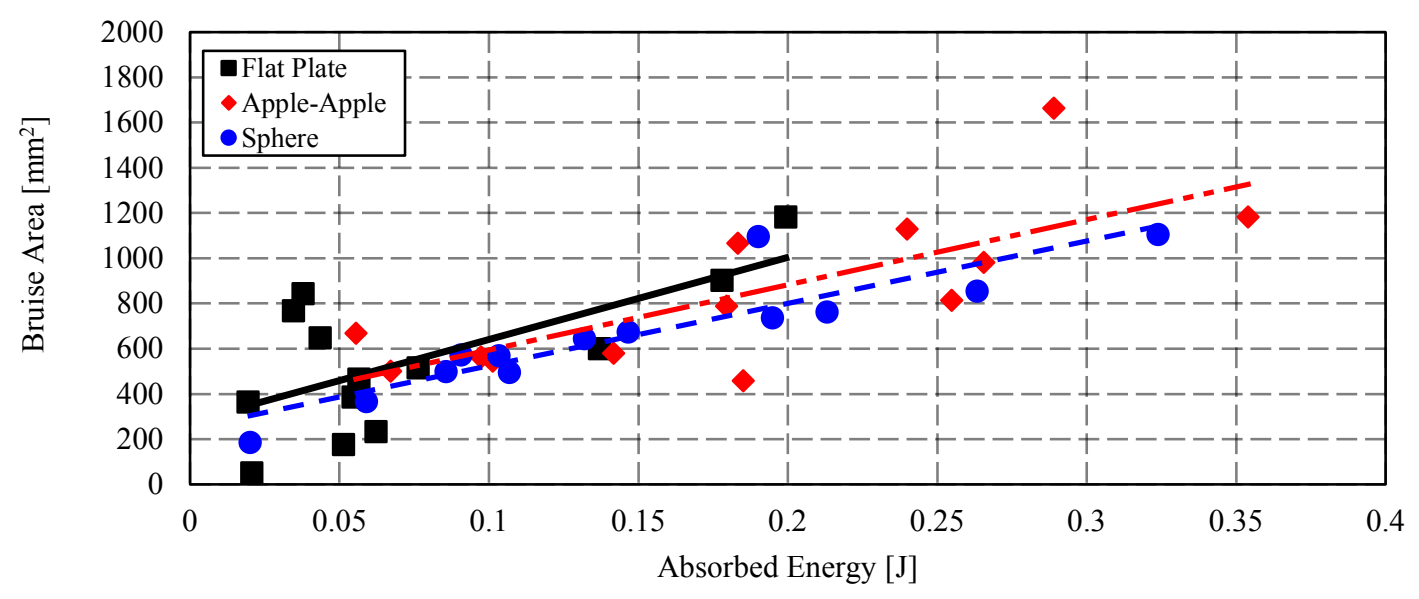

Figure C.5: Correlation between absorbed energy and BA for spherical, flat plate and apple impactors

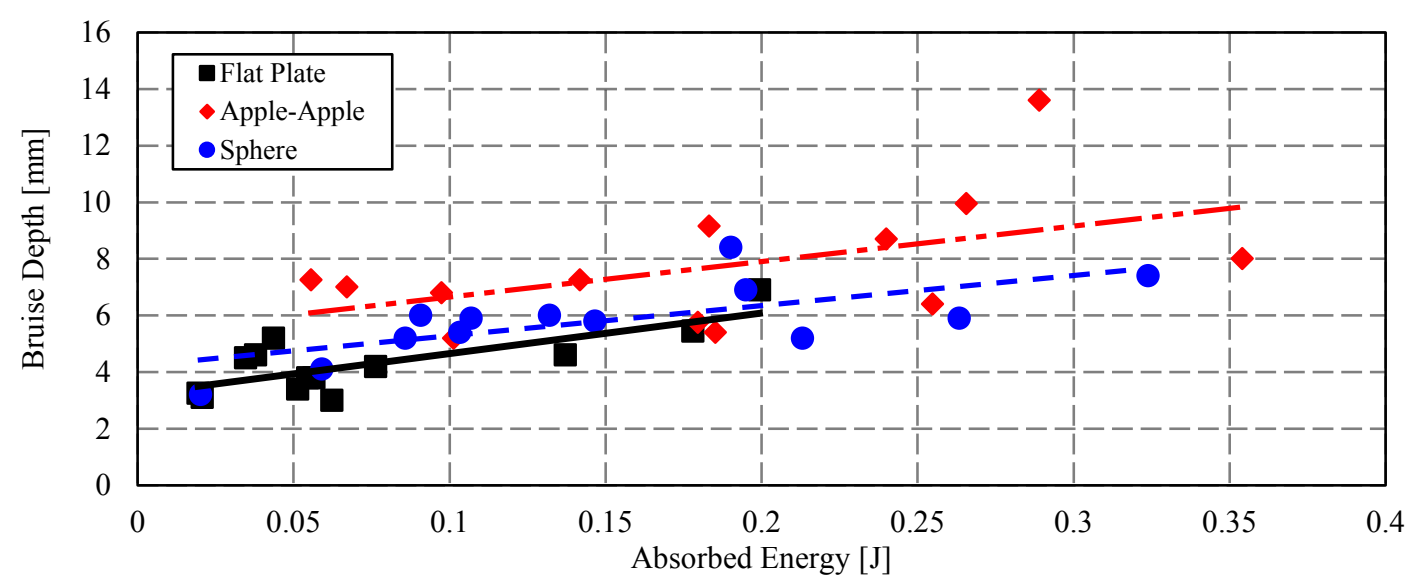

Figure C.6: Correlation between absorbed energy and BD for spherical, flat plate and apple impactors 


\section{APPENDIX D: ALGORITHM FLOW CHARTS}

In order to support the development of the algorithms discussed in Chapter 5 additional flow charts are presented in this chapter. The use of flow charts to graphically represent algorithms or portions of algorithms assists in the development and clarification of programme control structures and functionality. The flow charts shown in this chapter accompany the programme pseudocode given in Chapter 5 .

The flow chart presented in Figure D. 1 is paired to the pseudocode presented Figure 5.7 for the coupling of the impact force to the resulting bruise damage in the DEM programme. Figure D.2 accompanies the pseudocode given in Figure 5.6 which details the single contact assignment algorithm. The basic outline for the implementation of the bruise overlap formulation is presented with pseudocode in Figure 5.8. The programme sequence, control and selection structure is further clarified graphically in Figure D.3. The development of a bruise visualisation algorithm is presented in Chapter 6.6. The algorithm is relatively straightforward and makes use of the extra variables that $\mathrm{FISH}$ allows the user to access within the existing pebble class structure. The algorithm structure is graphically presented in Figure D.5 and is similar to the algorithm operation described in Chapter 5.5

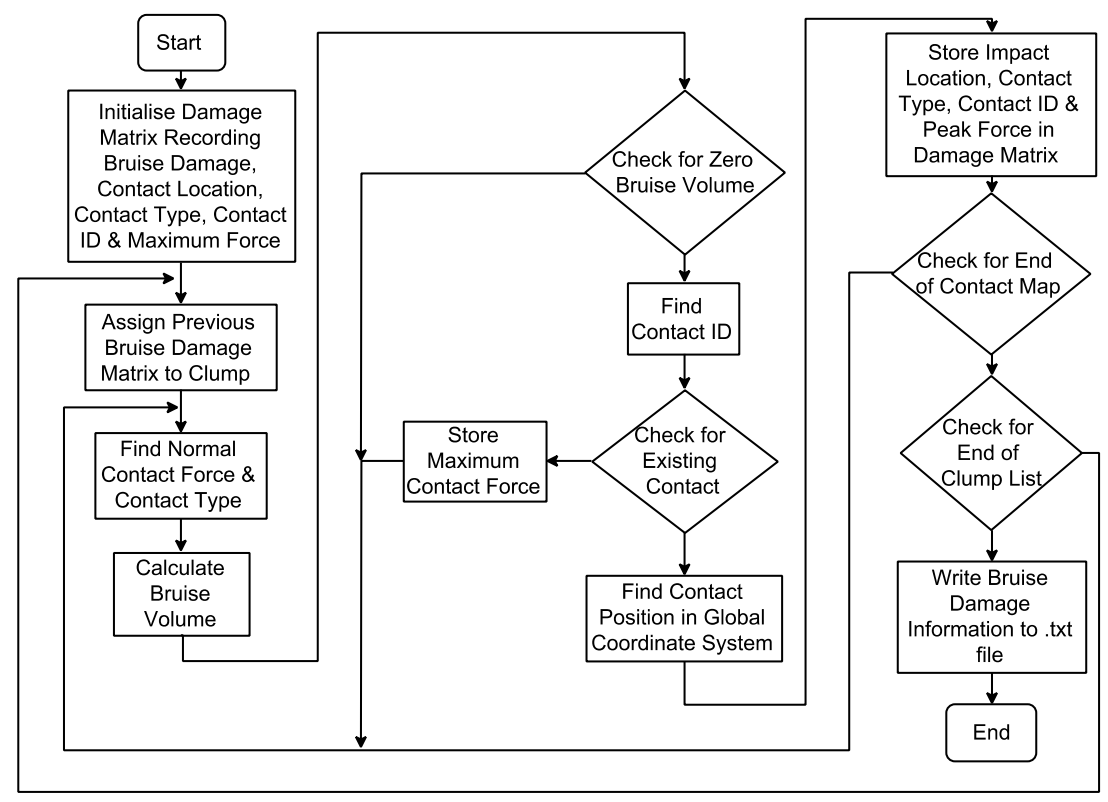

Figure D.1: Basic algorithm outline for obtaining contact data 


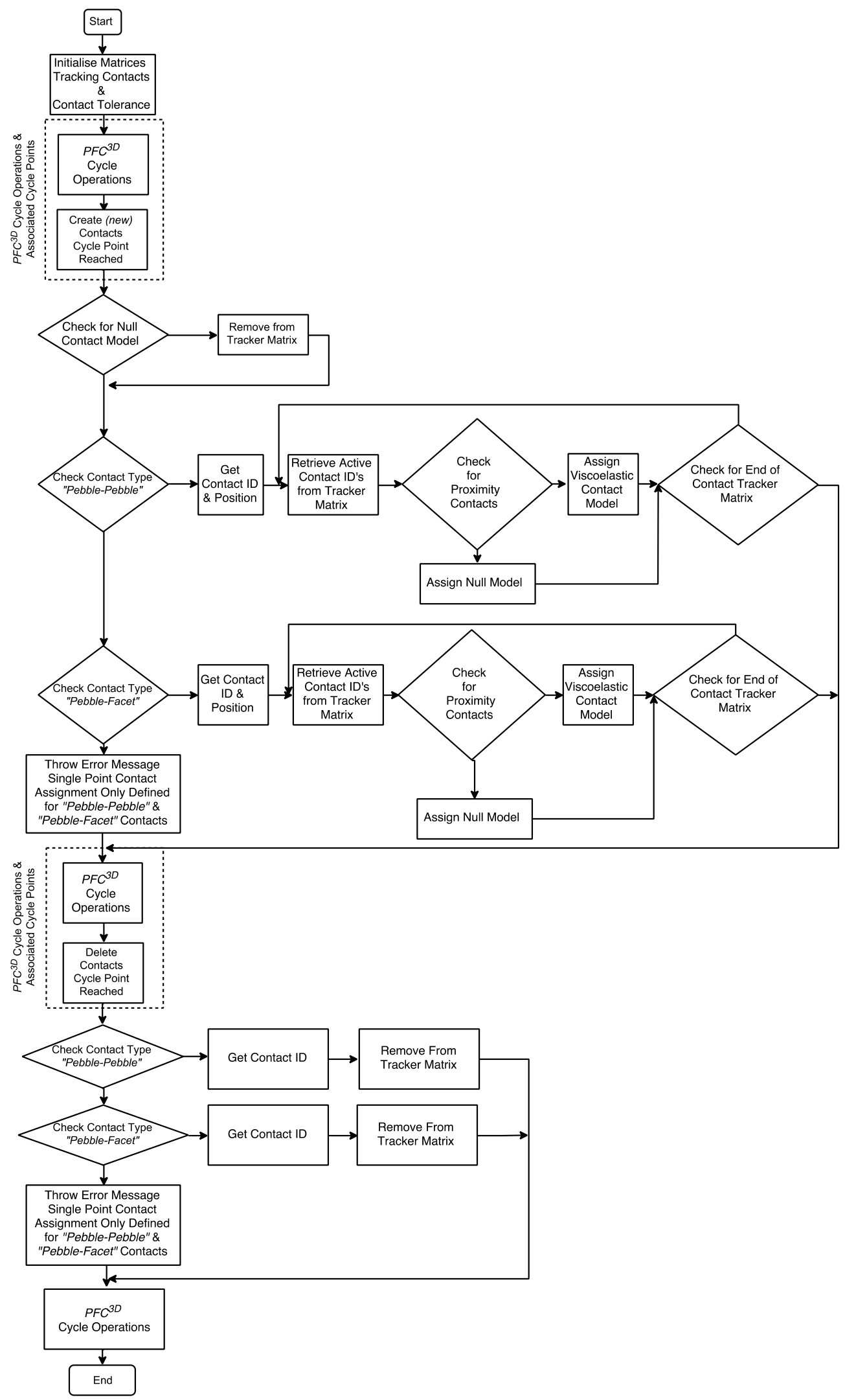

Figure D.2: Basic algorithm outline for assigning and removing single contacts 


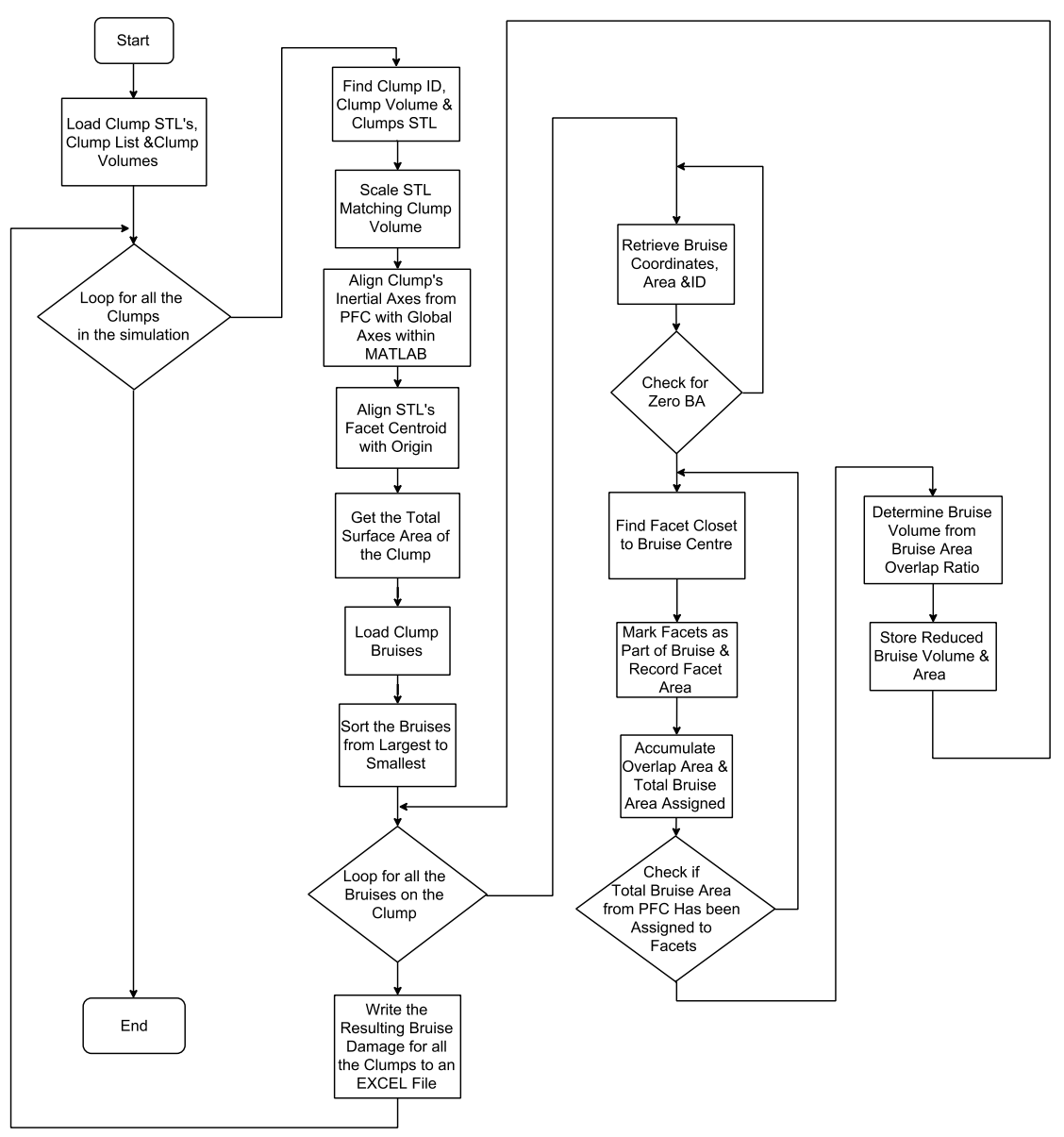

Figure D.3: Basic algorithm outline implementing numerical overlap

The bruise overlap formulation is detailed in Section 5.6. Figure D.3 shows the accompanying flow chart for the pseudocode of the bruise overlap formulation given in Figure 5.8. Additionally, the visualisation of apple STL meshes, comprised of multiple bruises, as depicted in the post-process environment is given in Figure D.4.
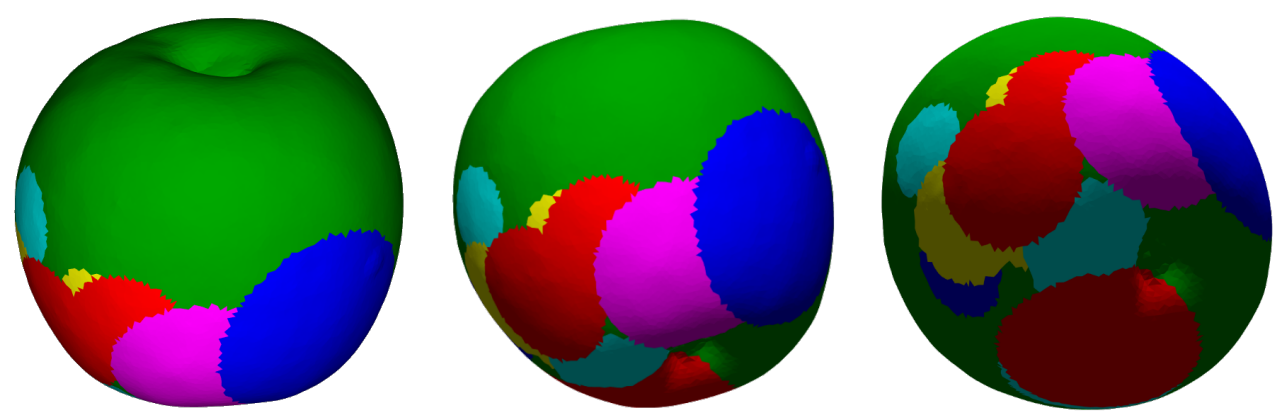

Figure D.4: Resulting BA as visualised on the post-process level 


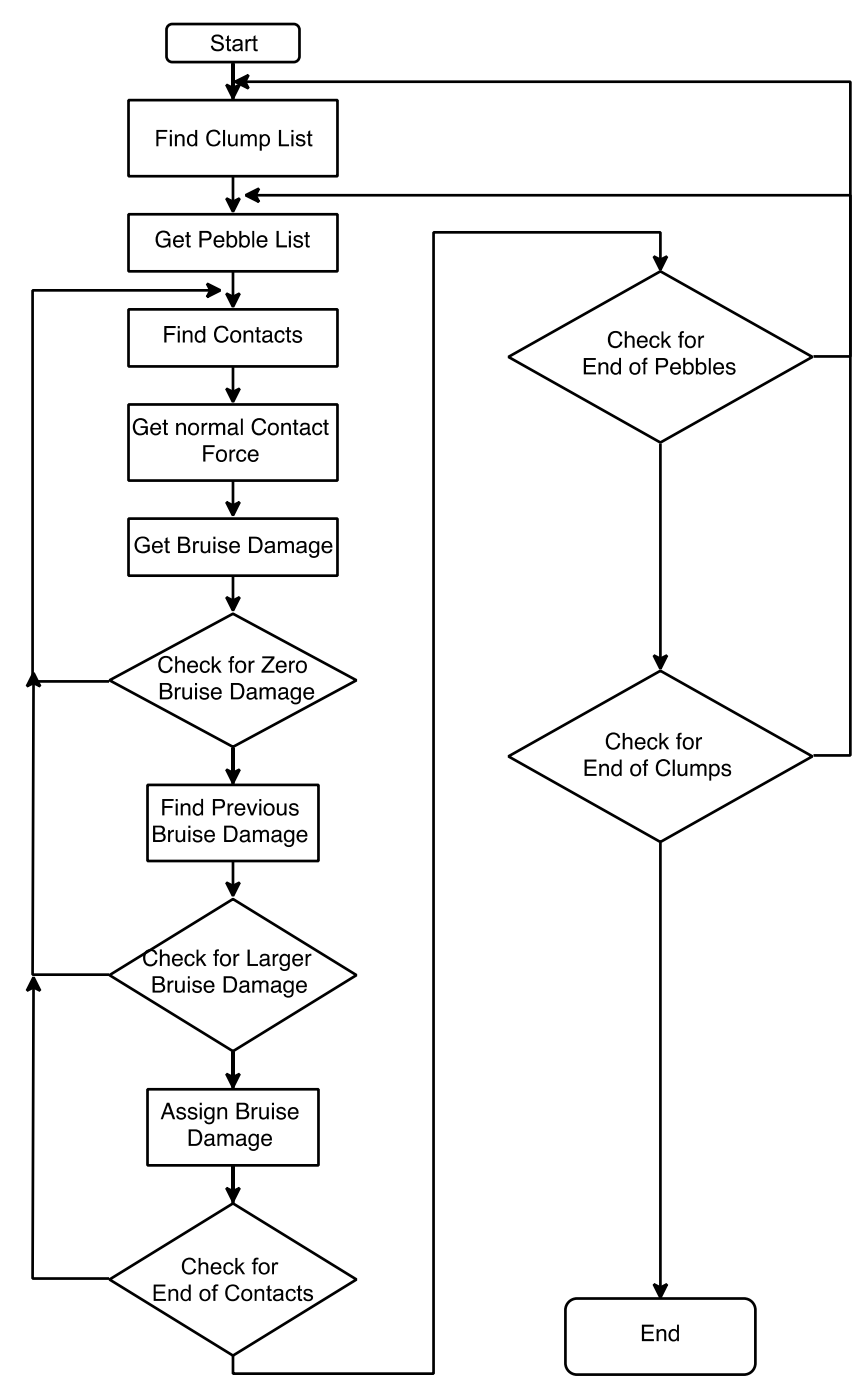

Figure D.5: Basic algorithm outline depicting the bruise visualisation algorithm

\section{APPENDIX E: DENSITY ACQUISITION}

Accurate attainment of the density and specific gravity of materials involved in the agricultural sector is important for applications within the food industry along with the ever-expanding application of DEM into the agricultural sector. DEM requires precise knowledge of the density to define the mass properties of the particles. Typically a uniform density is specified which is used in combination with the surface description of the particle to determine the mass, centroid position and inertia tensor. To this end, a method that is capable of correctly determining the density of irregular granular shaped products is required. 
Most agricultural products are of a highly irregular shape. This makes the precise calculation of their volume impossible. Accordingly, the method of water displacement, first described by Archimedes, was used. Thirty apple specimens were first weighed on a calibrated scale (accurate to within $0.0002 \mathrm{~g}$ ). Subsequently, they were completely submerged in a $90 \times 90 \times 180 \mathrm{~mm}$ acrylic box using a sinker rod. The resulting increase in the water level $(\Delta \mathrm{H})$ was measured with a Vernier gauge and the displaced water volume could be found. Consequently, the density could be determined by dividing the apple mass by the displaced volume. The method is similar to that described by Mohsenin (1986:95) and an illustration of the experimental set-up is given in Figure E.1. The resulting mean density of the thirty GD apple specimens was found as $778 \mathrm{~kg} / \mathrm{m}^{3}$. This is in accordance with the values reported in literature of $750-840 \mathrm{~kg} / \mathrm{m}^{3}$ for GD apples by Mohsenin (1986:787).

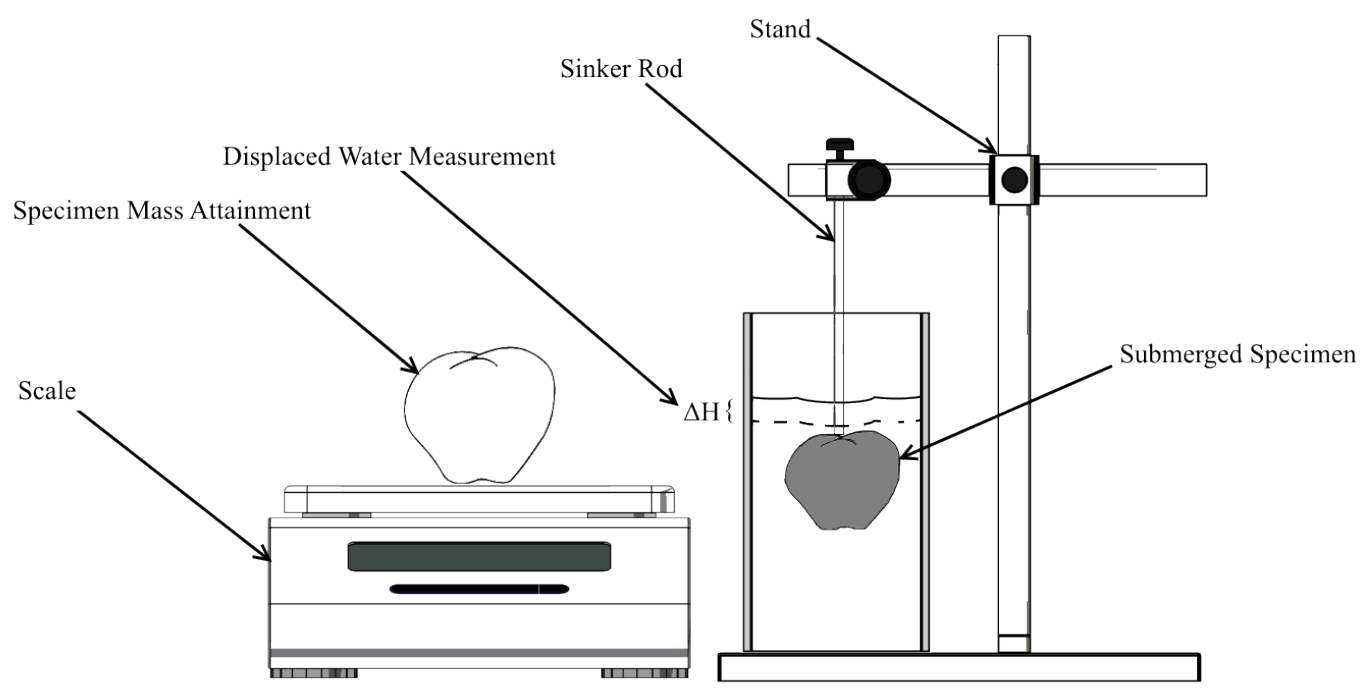

Figure E.1: Density measurement of apples

\section{APPENDIX F: MODEL VALIDATION OUTPUTS}

The validation of the derived contact model is conducted in Chapter 6. The nature of DEM allows for the dynamic mechanical behaviour of various particle systems to be animated. Consequently, the validation procedure produced many visual outputs. In order to streamline the structure of Chapter 6, supplementary model outputs supporting the model validation are presented. 


\section{APPENDIX F: MODEL VALIDATION OUTPUTS}

The qualitative visual comparison for the structured packing arrangement, in the vibration box, is presented in Figure 6.11. The technique illustrated Figure 6.11 did not allow for quantitative comparisons between experimental and simulation bruise damage to be made. Accordingly, the structured packing arrangement was repeated with regular apples, as shown in Figure F.1, which was used for the quantitative comparison.

(a)
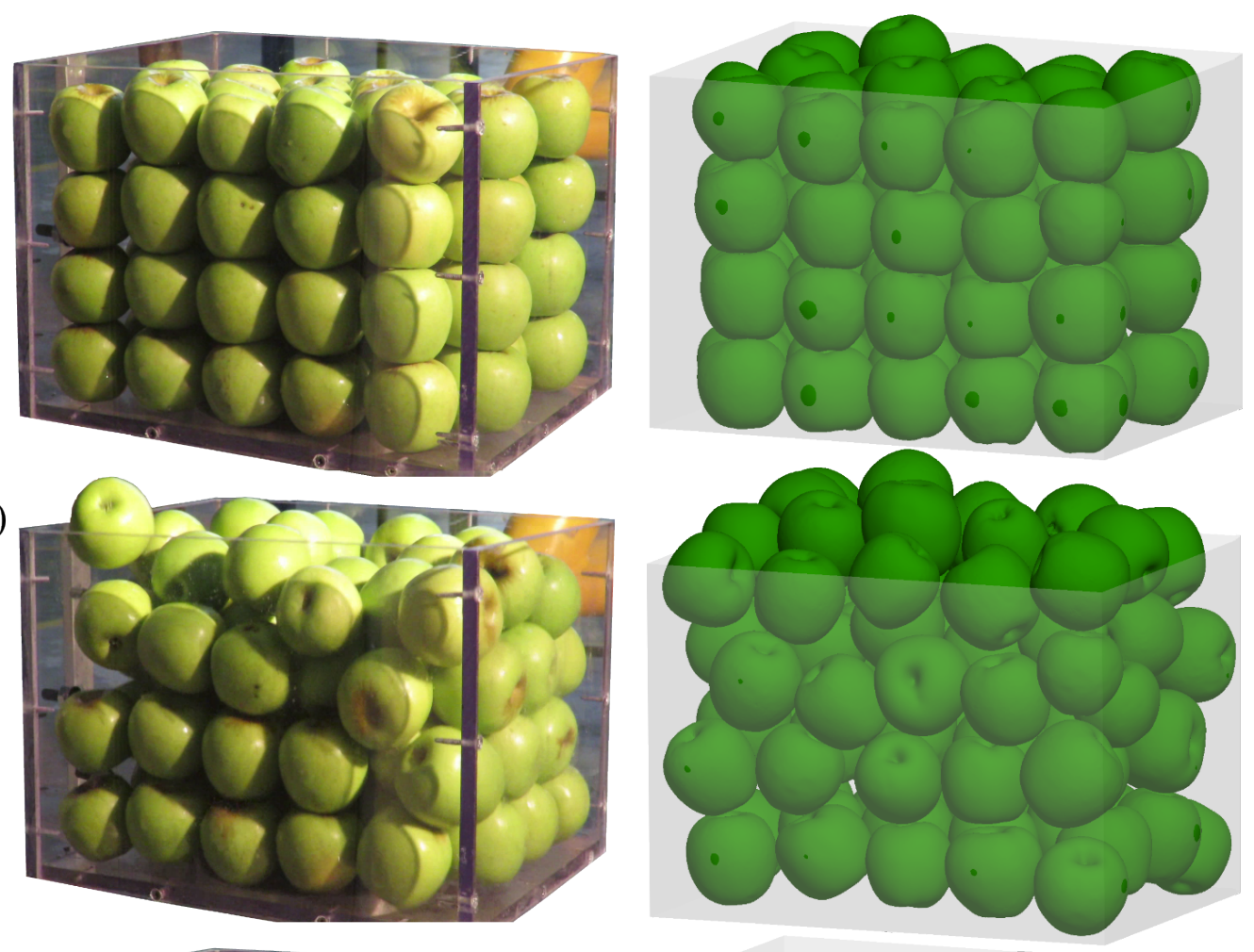

(c)
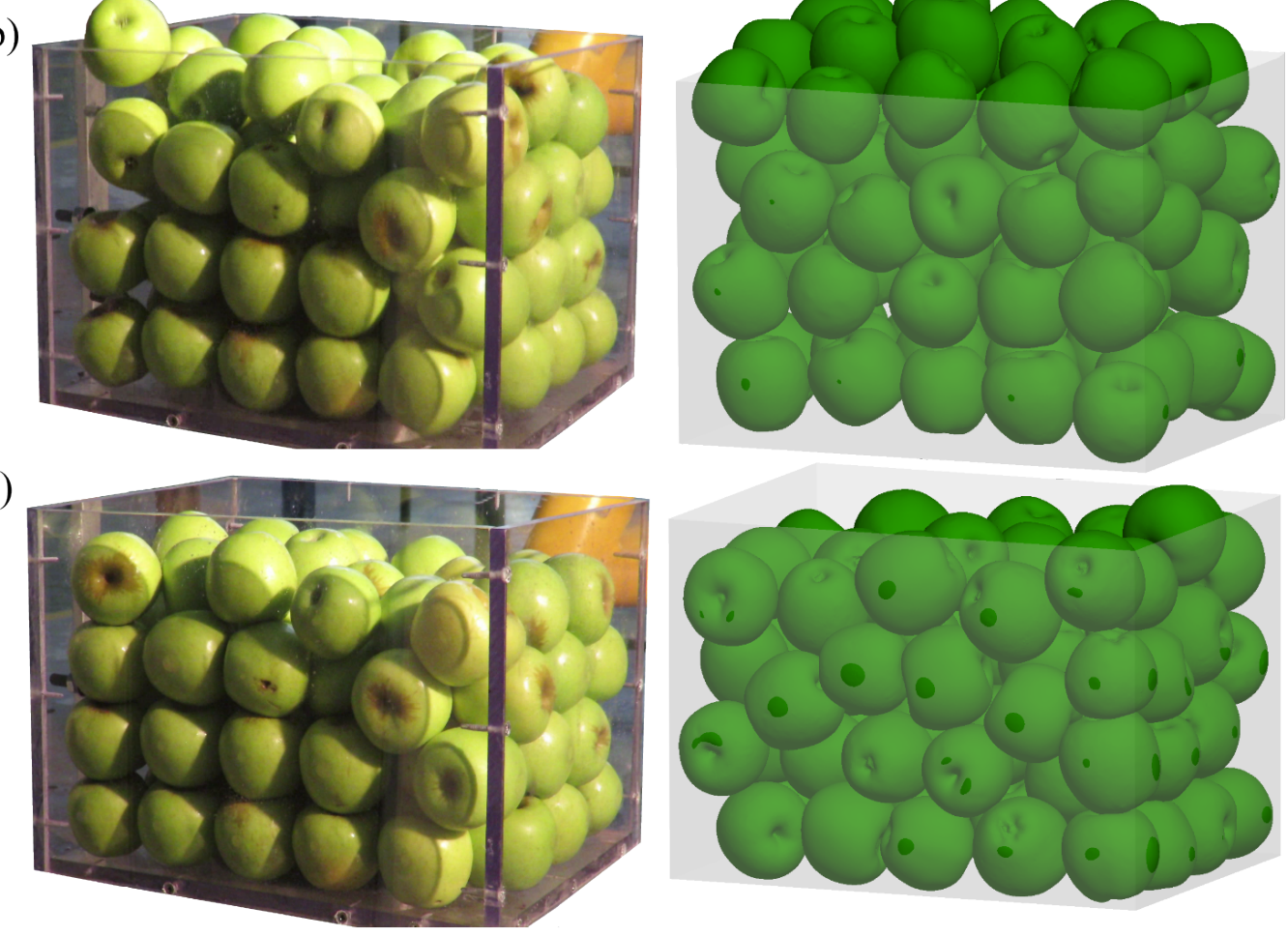

Figure F.1: Structured packing in the vibration box used for the quantitative comparison between simulation and experiment, (a) at the start, (b) after 30 seconds and (c) after 60 seconds 


\section{APPENDIX F: MODEL VALIDATION OUTPUTS}
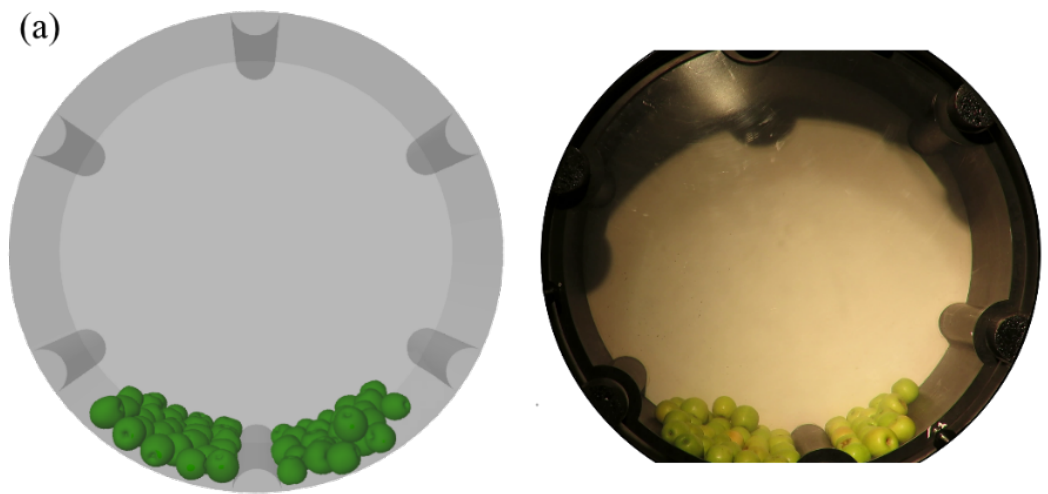

(b)
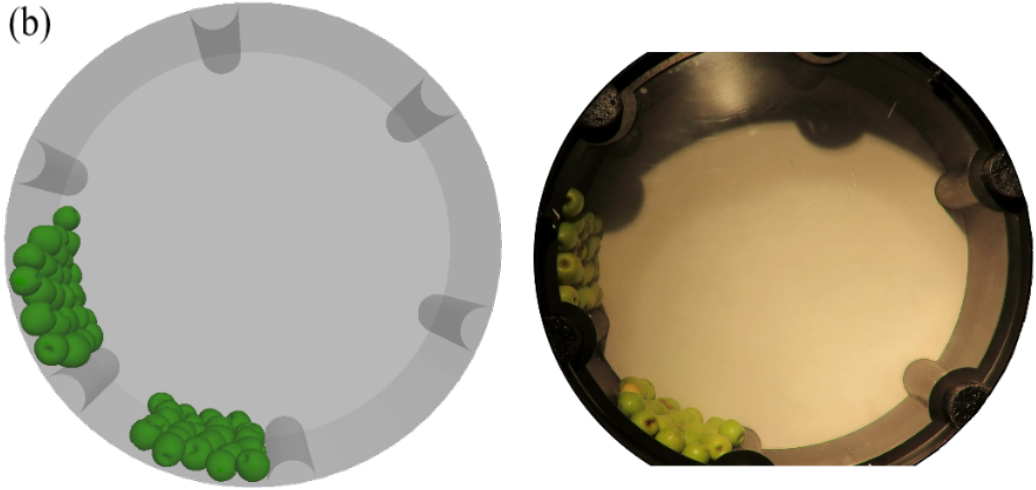

(c)
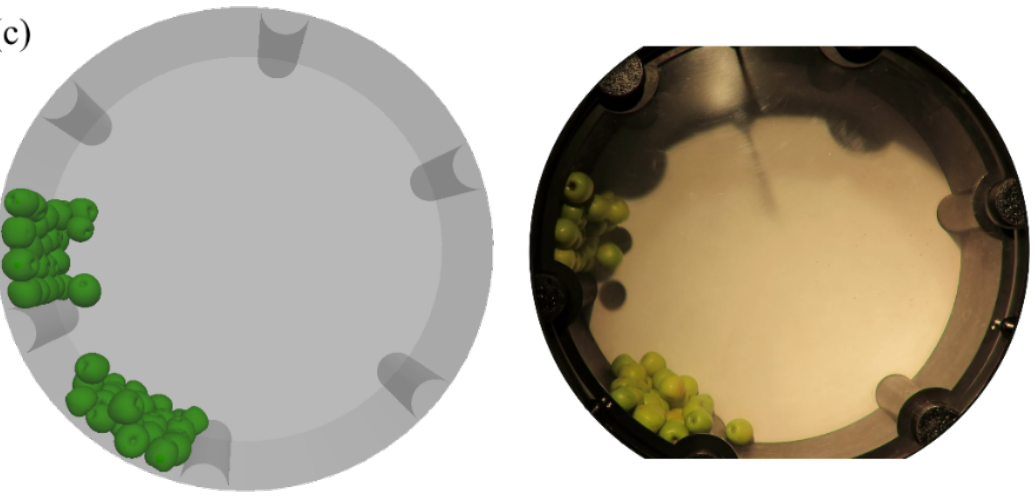

(d)
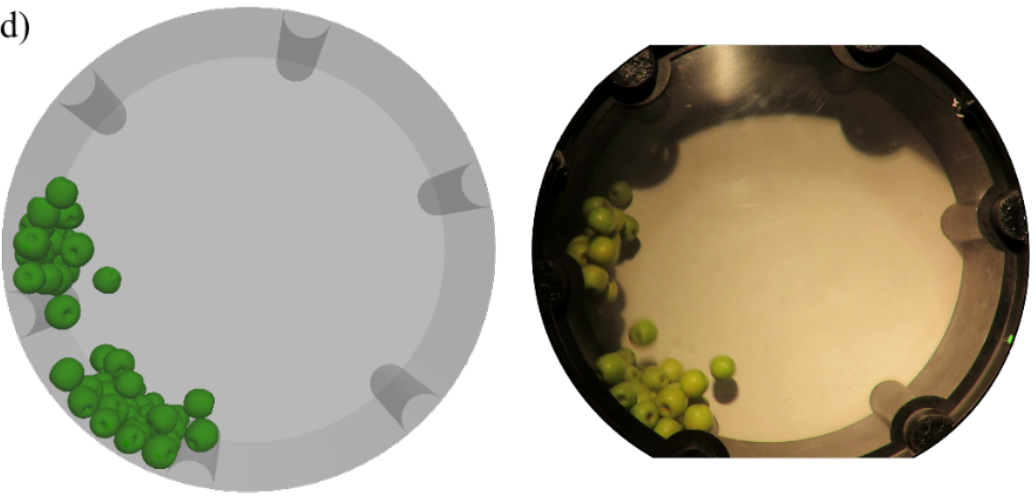

Figure F.2: Visual comparison between simulation and experiment with the rotating drum set at $400 \mathrm{~mm}$ depth 
The rotating drum validation experiment made use of two different depth settings within the drum. The first experiment made use of the drum set at a depth of $200 \mathrm{~mm}$ as shown in Figure 6.8. For the second experiment, the drum was set at a depth of $400 \mathrm{~mm}$ and to supplement the experimental results and discussion of Section 6.4 the qualitative visual comparison between the experiment and simulation is presented in Figure F.2.

\section{APPENDIX G: BRUISE DAMAGE SENSITIVITY}

A sensitivity study on how variations in the magnitudes of the main input parameters affect the model outputs is presented in this section. In order to expedite the investigation, the results of the rotating drum, set at a depth of $200 \mathrm{~mm}$, were used. Subsequently, confidence in the input parameters specified for the single contact assignment algorithm (described in Section 5.4) and the elastic stiffness and damping constants (derived in Section 3.5) is increased. This will help test the robustness of the derived model to variations that are expected to occur due to the inherent nature of biological materials.

\section{G.1. Sensitivity of the Contact Proximity Radius}

The uncertainty of the contact proximity radius (hence PR), described in Section 5.4, was investigated. Within this study, the contact PR was set at $7.5 \mathrm{~mm}$ ( $\varnothing 15 \mathrm{~mm}$ ), which was close to the mean bruise widths measured for the bruise prediction model. Subsequently, three cases were used to explore the sensitivity of the model to the PR. The first case made use of a radius of $5 \mathrm{~mm}$, the second a radius of $10 \mathrm{~mm}$ and lastly, an extreme case making use of only balls (single spheres) - modelling the effect of only allowing one (active) contact to be registered between two contacting pieces.

The influence of a $33 \%$ increase or decrease of the PR on the resulting bruise damage distribution is shown in Figure G.1. Additionally, a comparison with the simulation comprised of only balls is supplied in Figure G.1. No significant difference in the bruise damage distributions was found for either of the three cases. Furthermore, the mean bruise damage per apple for the experiments and simulations is compared in Table G.1. The percentage differences relative to the 
experimental results are also given in Table G.1 Again, no significant variation in the resulting bruise damage was observed. Consequently, large variations in the contact PR produced relatively little change in the model output. This showed that the model was quite robust and even in the extreme case where only one active contact between contacting pieces could exist (i.e. spheres/balls), no significant variations occurred within the bruise damage distributions or the mean bruise damage per clump.
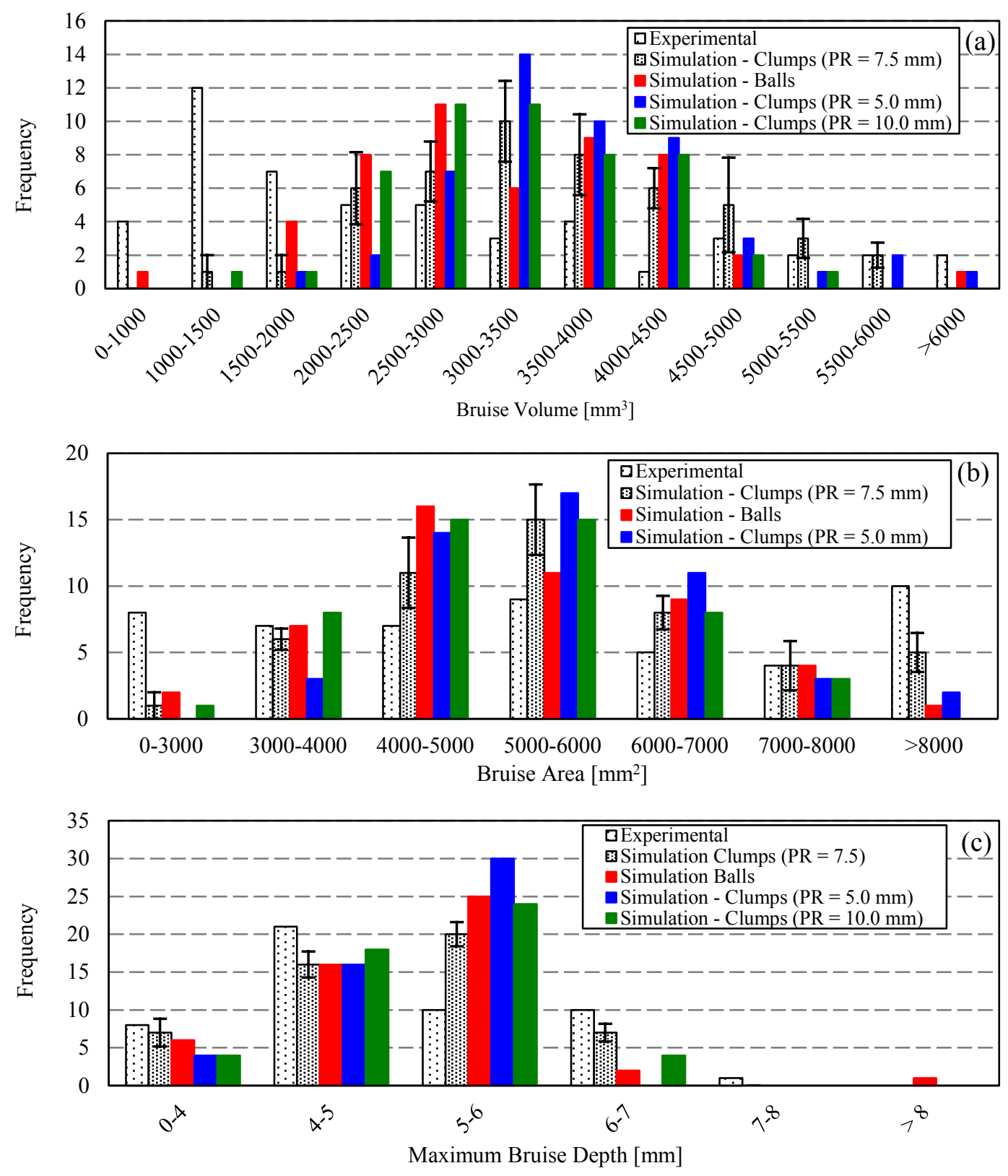

Figure G.1: Sensitivity of the contact proximity radius for the rotating drum set at $200 \mathrm{~mm}$ depth, (a) BV, (b) BA and (c) maximum BD respectively 
Table G.1: Sensitivity of bruise damage for the rotating drum set at $200 \mathrm{~mm}$ depth with regards to the contact proximity radius. Percentage difference is given relative to the experiment.

\begin{tabular}{|c|c|c|c|c|c|c|}
\hline Bruise Damage & $\begin{array}{c}\text { BV } \\
{\left[\mathrm{mm}^{3}\right]}\end{array}$ & $\begin{array}{c}\% \\
\text { Difference }\end{array}$ & $\begin{array}{c}\text { BA } \\
{\left[\mathrm{mm}^{2}\right]}\end{array}$ & $\begin{array}{c}\% \\
\text { Difference }\end{array}$ & $\begin{array}{c}\text { BD } \\
{[\mathrm{mm}]}\end{array}$ & $\begin{array}{c}\% \\
\text { Difference }\end{array}$ \\
\hline Experiment $[\bar{x}]$ & 2728 & {$[-]$} & 5837 & {$[-]$} & 4.9 & {$[-]$} \\
\hline $\begin{array}{l}\text { Simulation }[\bar{x}]- \\
\mathrm{PR}=7.5 \mathrm{~mm}\end{array}$ & 3583 & $31 \%$ & 5518 & $-5.5 \%$ & 5.1 & $4.1 \%$ \\
\hline $\begin{array}{l}\text { Simulation }[\bar{x}]- \\
P R=5.0 \mathrm{~mm}\end{array}$ & 3704 & $36 \%$ & 5537 & $-5.1 \%$ & 5.0 & $2.0 \%$ \\
\hline $\begin{array}{l}\text { Simulation }[\bar{x}]- \\
P R=10.0 \mathrm{~mm}\end{array}$ & 3262 & $20 \%$ & 5091 & $-12.8 \%$ & 5.0 & $2.0 \%$ \\
\hline $\begin{array}{l}\text { Simulation }[\bar{x}]- \\
\text { Balls }\end{array}$ & 3282 & $20 \%$ & 5197 & $-11 \%$ & 5.2 & $6.1 \%$ \\
\hline
\end{tabular}

The vigorous mixing that occurred in the drum lead to contacts that were very scattered in terms of position and had relatively large magnitudes. Consequently, the uncertainty of the effect of multiple contacts on the same position (resulting in a large degree of overlap) could not be quantified. To this end, a simulation was conducted for the vibration box comprised of spheres/balls instead of clumps. It was observed that the balls exhibited a large degree of random rotations within the vibration box. As discussed in Section 6.6 clumps tended to settle and rotate around their major equatorial axes. This is illustrated in Figure G.2 where the mean rotations $(\bar{\omega})$ around the global coordinate system is compared between balls and clumps.
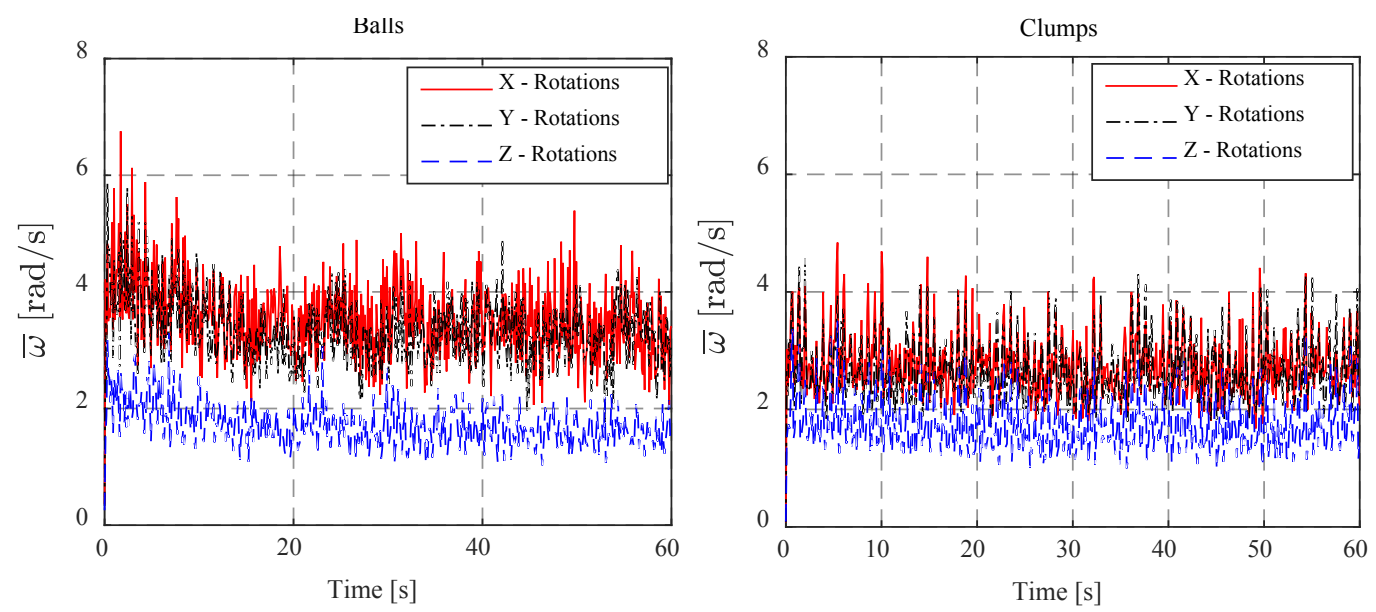

Figure G.2: Comparisons between the mean rotations of balls \& clumps for the vibration box 


\section{APPENDIX G: BRUISE DAMAGE SENSITIVITY}

From Figure G.2 it is observed that the clumps tended to have $\pm 20 \%$ fewer rotations in the $x$ and $y$ axes, compared to that of the balls, while rotations in the $z$ axis (the direction in which the box was excited) were approximately equal. The decrease in the clumps rotations was attributed to the clump centroid not being located at its geometric centre and the shape of the apple. As a result, a band of multiple overlapping contacts occurred around the clumps' major axes, as was observed in Figure 6.17. Accordingly, the balls were completely covered in contacts whereas the contacts on the clumps occurred relatively close to one another. A quantitative comparison between the mean number of contacts and bruise damage before and after overlap is given in Table G.2. From Table G.2 it is observed that a much larger number of contacts and amount of bruise damage was present before and after the overlap formulation for the balls. Additionally, the reduction in the number of contacts due to the overlap formulation is presented.

Table G.2: Comparison between the reduction in number of contacts and bruise damage before and after the overlap formulation

\begin{tabular}{c|ccc}
$\begin{array}{c}\text { Bruise Damage \& } \\
\text { Contacts }\end{array}$ & Experimental & Clumps & Balls \\
\cline { 1 - 1 } Before Overlap & & & \\
\cline { 1 - 1 } No. of Contacts $[\overline{\boldsymbol{x}}]$ & & 91 & 358 \\
BV $[\overline{\boldsymbol{x}}]$ & & 6254 & 77590 \\
BA $[\bar{x}]$ & & 16953 & 121929 \\
BD $[\overline{\boldsymbol{x}}]$ & & 2.4 & 5.0 \\
After Overlap & & & \\
\cline { 1 - 1 } No. of Contacts $[\overline{\boldsymbol{x}}]$ & 5 & 53 & 101 \\
BV $[\overline{\boldsymbol{x}}]$ & 1197 & 1626 & 10517 \\
BA $[\overline{\boldsymbol{x}}]$ & 3791 & 4697 & 15194 \\
BD $[\bar{x}]$ & 3.0 & 2.4 & 5.0 \\
$\%$ Reduction & & & \\
\cline { 1 - 1 } No. of Contacts $[\overline{\boldsymbol{x}}]$ & & $42 \%$ & $72 \%$ \\
BV $[\bar{x}]$ & & $74 \%$ & $86 \%$ \\
BA $[\bar{x}]$ & & $73 \%$ & $88 \%$ \\
BD $[\bar{x}]$ & & {$[-]$} & {$[-]$}
\end{tabular}

Consequently, the model was shown to be sensitive to accurate shape representation. For the case of the rotating drum, the model was shown not to be sensitive to the PR or shape representation but for the vibration box, the model was shown to be very sensitive to accurate shape representation. The superior shape representation of the clumps produced the most accurate simulation results regardless of application. DEM is a predictive tool and the sensitivity of a particular system to shape representation is not always known. However, the use of clumps 


\section{APPENDIX G: BRUISE DAMAGE SENSITIVITY}

to accurately represent the particles should produce the most accurate results independent of the particular case under investigation.

\section{G.2. Sensitivity of the Contact Parameters}

The sensitivity of the elastic moduli and damping constants was next explored. From Chapter 3.5 it was observed that the model was more sensitive to the effective damping parameter between two apples, which required additional investigation. In order to determine the robustness of the developed model and the accuracy with which the elastic moduli and damping constant needs to be specified, a sensitivity analysis was conducted. The analysis commenced by systematically increasing or decreasing the contact parameters by $50 \%$ for the simulation involving the rotating drum set at a depth of $200 \mathrm{~mm}$.

Accordingly, the elastic moduli were increased by $50 \%$ for both contact situations while then damping constants were kept the same. Next, the elastic moduli were decreased by $50 \%$. The same variation was conducted for the damping constants. The resulting increase or decrease in bruise damage is presented in Table G.3, in addition to the differences between the mean bruise damage and experimental results. It is observed that the model was relatively sensitive to a decrease in the damping constant with large under-predictions for the BV, BA and BD witnessed. Similar results for a decrease in the elastic moduli were also observed. The model is shown to greatly over-predict the BV and BA for a $50 \%$ increase in moduli but only slightly over-predict the BD. A similar trend is shown for an increase in the damping constants.

Accordingly, the BV and BA was sensitive to an increase in the contact parameter constants but the $B D$ was not. The $B V, B A$ and $B D$ was sensitive to a decrease in the contact parameter constants. Large increases or decreases in contact model parameters resulted in large increases or decreases in BV and BA. Large increases in parameter constants only produced relatively small increases in BD. Large decreases in parameter constants also produced large decreases in BD. Consequently, the model's estimation of bruise damage is most sensitive to large decreases in parameter constants. The importance of obtaining the optimal combination of contact model parameters is thus highlighted. 
Table G.3: Comparison between a $50 \%$ increase/decrease in contact parameter magnitudes

\begin{tabular}{c|cc|cc|cc} 
Bruise Damage & $\begin{array}{c}\mathbf{B V} \\
{\left[\mathbf{m m}^{3}\right]}\end{array}$ & $\begin{array}{c}\% \\
\text { Difference }\end{array}$ & $\begin{array}{c}\text { BA } \\
{\left[\mathbf{m m}^{2}\right]}\end{array}$ & $\begin{array}{c}\% \\
\text { Difference }\end{array}$ & $\begin{array}{c}\text { BD } \\
{[\mathbf{m m}]}\end{array}$ & $\begin{array}{c}\% \\
\text { Difference }\end{array}$ \\
\cline { 1 - 5 } Experiment $[\bar{x}]$ & 2728 & {$[-]$} & 5837 & {$[-]$} & 4.9 & {$[-]$} \\
\hline \hline Optimal $[\bar{x}]$ & 3583 & $31 \%$ & 5518 & $-5.5 \%$ & 5.1 & $4.1 \%$ \\
\hline $\begin{array}{c}50 \% \text { increase in } \\
\text { moduli }[\bar{x}]\end{array}$ & 6013 & $120 \%$ & 8454 & $45 \%$ & 6.0 & $18 \%$ \\
$\begin{array}{c}50 \% \text { decrease in } \\
\text { moduli }[\bar{x}]\end{array}$ & 757 & $-72 \%$ & 1581 & $-73 \%$ & 3.2 & $-35 \%$ \\
$\begin{array}{c}50 \% \text { increase in } \\
\text { damping } \\
\text { parameter }[\bar{x}]\end{array}$ & 5761 & $111 \%$ & 8158 & $40 \%$ & 5.6 & $14 \%$ \\
$\begin{array}{c}50 \% \text { decrease in } \\
\text { damping } \\
\text { parameter }[\bar{x}]\end{array}$ & 628 & $-77 \%$ & 1274 & $-78 \%$ & 2.9 & $-41 \%$
\end{tabular}

\section{APPENDIX H: SAMPLE APPLICATIONS}

The developed contact model provides the user with the ability to investigate various fruits that are susceptible to mechanical damage. In order to illustrate the capability of $P F C^{3 D}$ to investigate the potential mechanical damage that may occur to various fruit and vegetables the representation of some produce shapes is illustrated in Figure $\mathrm{H} .1$ and Figure H.2. The representation of tomatoes in $P F C^{3 D}$ is shown in Figure H.1a with pomegranates presented in Figure H.1b. Furthermore, bananas are represented in Figure $\mathrm{H} .2 \mathrm{a}$, pears in Figure $\mathrm{H} .2 \mathrm{~b}$ and potatoes in Figure H.2c.

The potential application of the developed model in future research is illustrated in Figure H.3 where apples are represented in their packaging layers in a transport box. The box was modelled as rigid, but the trays were modelled by elastically bonding elements together to form a flexible realistic representation. This allows for the effect of different packaging materials, or material combinations, to be studied under varying transport conditions before any physical implementation or experimentation commences. Furthermore, the implementation of the developed model for apple packing plants is illustrated in Figure H.4. The example application 


\section{APPENDIX H: SAMPLE APPLICATIONS}

is given where apples were loaded from a bin onto a conveyor and then washed in a water fluid conveyor.

(a)

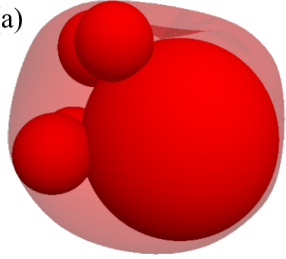

(b)

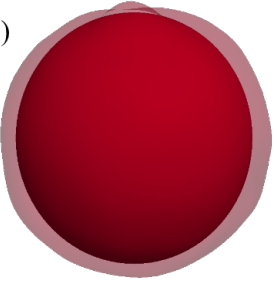

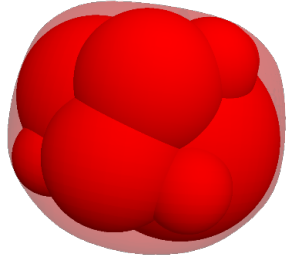

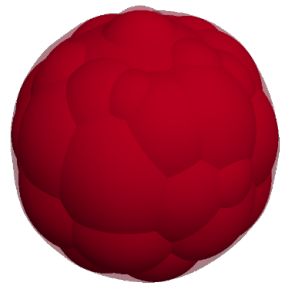

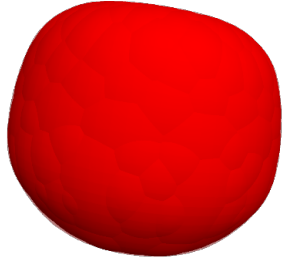

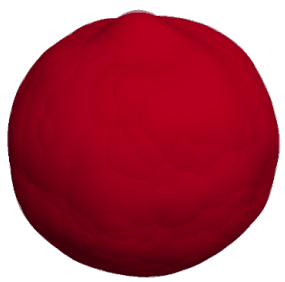

Figure H.1: Fruit \& vegetable representation in $P F C^{3 D}$ using clumps, (a) models a tomato, (b) models a pomegranate

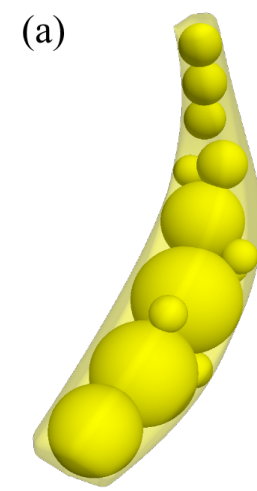

(b)

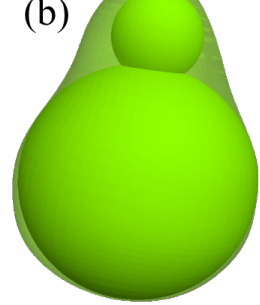

(c)

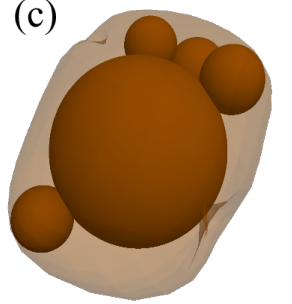

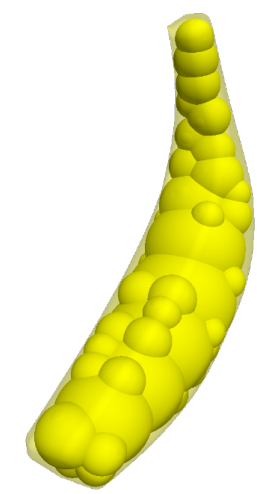
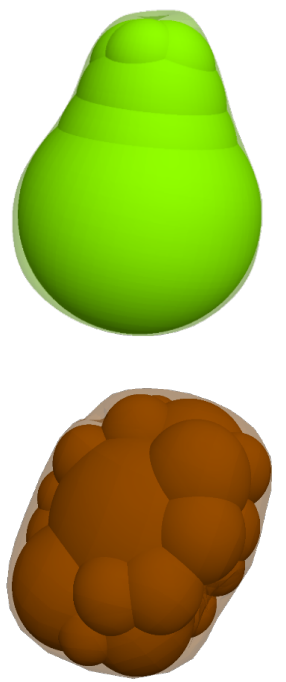
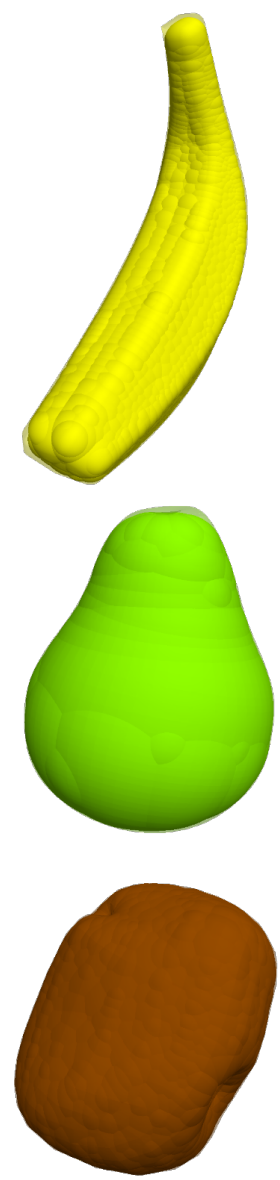

Figure H.2: Fruit \& vegetable representation in $P F C^{3 D}$ using clumps, (a) models a banana, (b) models a pear, (c) models a potato 
APPENDIX H: SAMPLE APPLICATIONS

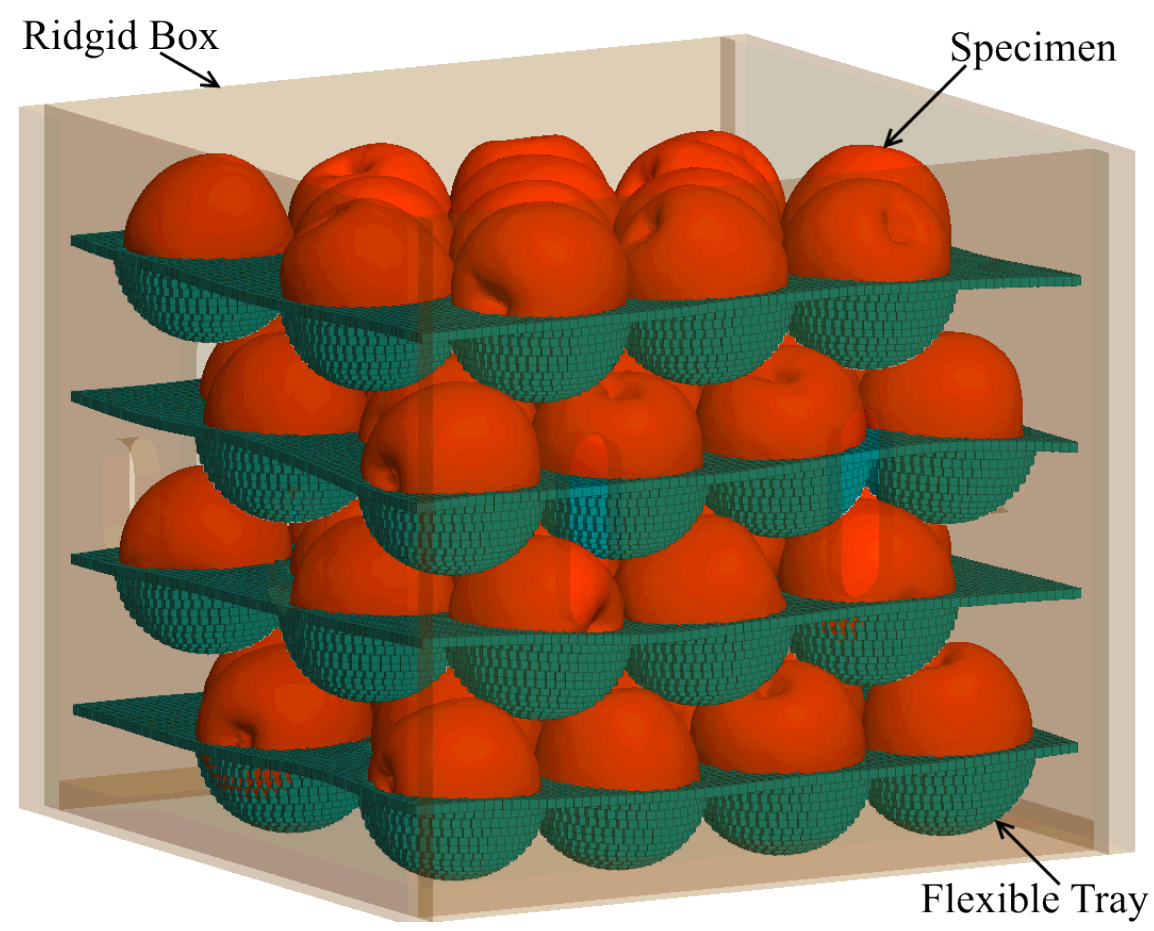

Figure H.3: Apple box with packaging material represented in $P F C^{3 D}$

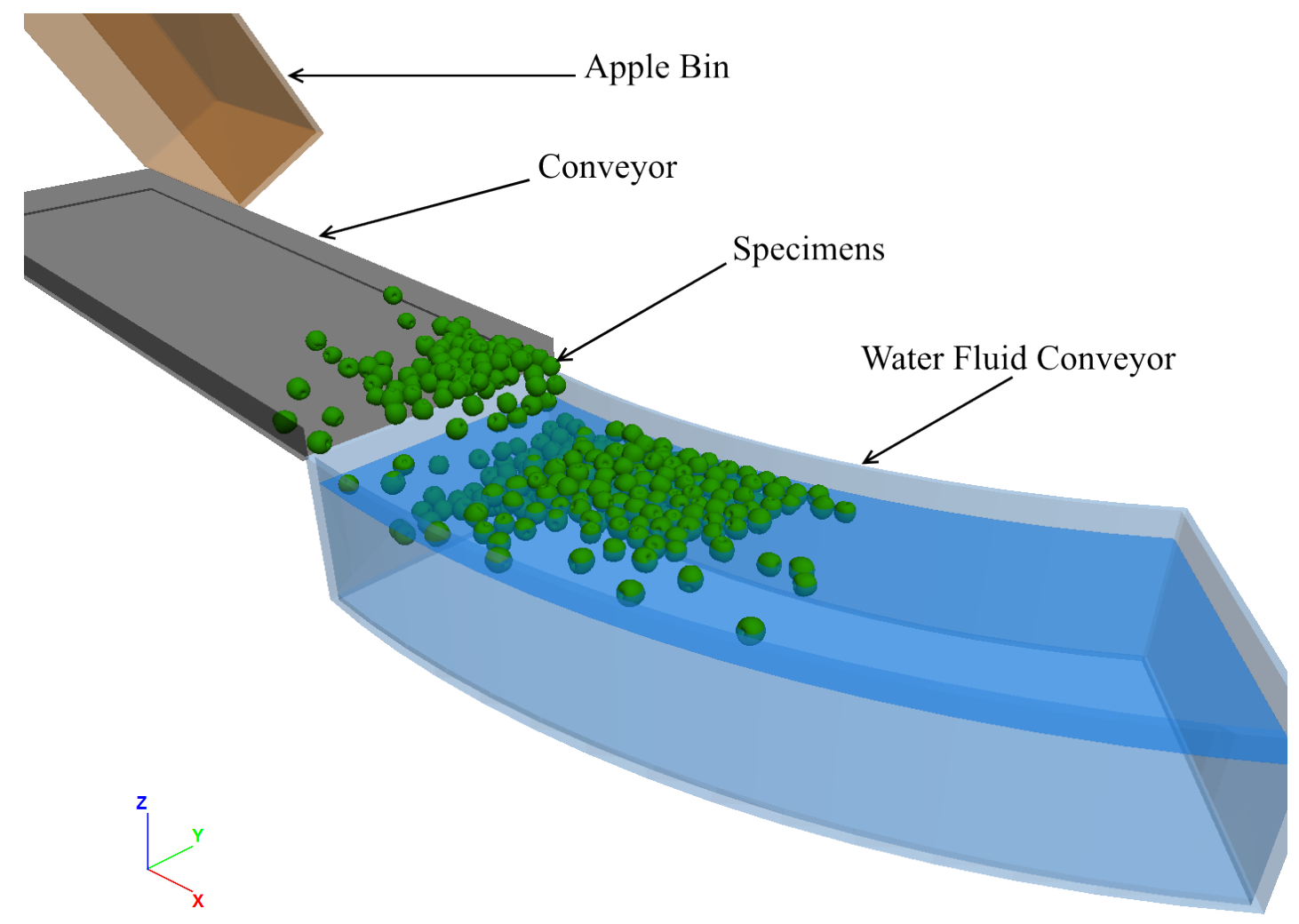

Figure H.4: Representation of an apple packing plant and water fluid conveyor 